UNIVERSIDADE DE SÃO PAULO

INSTITUTO DE FÍSICA

\title{
Equações de transporte na eletrodinâmica quântica não-comutativa
}

\author{
SAUlo HenRiQue PEREIRA
}

Orientador:

PROF. DR. JOSIF FRENKEL

Tese de doutorado apresentada ao Instituto de Física da Universidade de São Paulo, como parte dos requisitos para a obtenção do título de Doutor em Ciências.

BANCA EXAMINADORA:

PROF. DR. JOSIF FRENKEL - IFUSP

Prof. DR. AdILSON J. DA SILVA - IFUSP

Prof. Dr. Marcelo O. C. Gomes - IFUSP

Prof. Dr. Francisco CASTILho AlCARAZ - IFSC/USP

Prof. Dr. José AdEMIR SALES DE LIMA - IAG/USP 

Aos meus avós, com carinho e respeito 


$$
* * *
$$


Às vezes penso a Física como sendo uma grande e majestosa árvore. As raízes são as teorias fundamentais, ou teorias de grande unificação, que embora saibamos que devam existir, elas não são visíveis a nós. O tronco são a teoria da relatividade, a mecânica quântica, o eletromagnetismo e a termodinâmica, das quais muitos galhos se ramificam, dos mais diversos tamanhos

$$
\text { e para todos os lados. }
$$

Fico triste quando penso que este trabalho é apenas uma pequena folha desta imensa árvore. Mas a tristeza logo passa ao me lembrar que uma árvore sem folhas não tem beleza alguma. 


$$
* * *
$$




\section{Agradecimentos}

Aos meus pais, pelo apoio e confiança.

Ao professor Josif Frenkel, pela orientação constante, sábia e paciente.

À CAPES, pelo apoio financeiro.

À COSEAS, em especial à assistente social Rosana, pela moradia concedida durante todos os anos deste projeto.

Aos professores Fernando Brandt e Ashok Das, pelas conversas sobre temperatura finita.

A todos do Departamento de Física-Matemática, secretárias, professores, técnicos e alunos, que de alguma forma me ajudaram nesta jornada.

Ao meu irmão e aos meus amigos, pela força.

E mais uma vez aos meus pais, por tudo. 


\section{Resumo}

Estudamos neste trabalho as contribuições de 1-loop da eletrodinâmica quântica não-comutativa a altas temperaturas. Obtivemos as amplitudes de $n$-pontos por meio do método de diagramas de Feynman e mostramos que os mesmos resultados podem ser obtidos pelo método das equações de transporte de Boltzmann. Em paralelo estudamos as massas de blindagem que seguem do setor não-comutativo da teoria no limite estático, assim como a ação efetiva em 1-loop que gera todas as funções de $n$-pontos com índices espaciais. Também estudamos a quantização do campo de gauge no espaço não-comutativo pelo método do campo de fundo, obtendo uma generalização da base de ondas planas que se transforma covariantemente. 


\section{Abstract}

In this work we study the 1-loop contributions for noncommutative electrodynamics at high temperature. We calculate the $n$-point amplitudes by the Feynman diagrams method and we show that the same results can be obtained by the method of Boltzmann transport equations. We also study the screening mass derived from the noncommutative sector in the static limit case and the effective generating functional that determine all the amplitudes at one loop with spatial indices only. We quantize noncommutative QED by the background field gauge method and obtain a generalization of plane waves that transforms covariantly. 


\section{Sumário}

$\begin{array}{lll}\text { Agradecimentos } & \text { i }\end{array}$

$\begin{array}{lll}\text { Resumo } & \text { ii }\end{array}$

$\begin{array}{lll}\text { Abstract } & \text { iii }\end{array}$

Introdução

1 Alguns Aspectos da Eletrodinâmica Quântica $\quad 7$

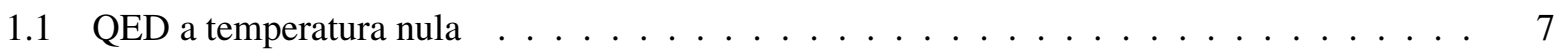

1.1.1 Lagrangeana e regras de Feynman da QED . . . . . . . . . . . . . . . . 8

1.1 .2 Seção de choque . . . . . . . . . . . . . . . . . . . . . . . 10

1.1 .3 Produção de múons . . . . . . . . . . . . . . . . . . . . . 11

1.1.4 Espalhamento coulombiano . . . . . . . . . . . . . . . 14

1.1.5 Funções de Green . . . . . . . . . . . . . . . . . . . . . . . . . . . . . . 17

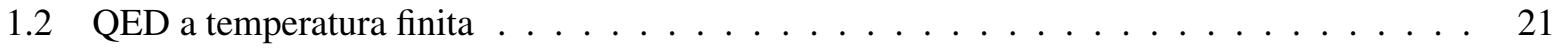

1.2.1 Revisão de termodinâmica . . . . . . . . . . . . . . . . . . 22

1.2.2 Funções de Green a temperatura finita . . . . . . . . . . . . . . . . . . . . 24

1.2 .3 Função de partição . . . . . . . . . . . . . . . . . . . . . . . . 25

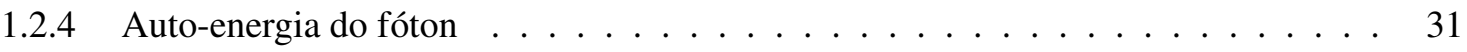

1.2.5 Blindagem dos campos elétrico e magnético em um plasma . . . . . . . . . 35

2 Teorias Não-Comutativas $\quad 39$

2.1 Não-comutatividade na física clássica $\ldots \ldots \ldots \ldots \ldots$. . . . . . . . . . 40

2.1.1 Partícula carregada em um campo magnético intenso e uniforme . . . . . . . . . . 41

2.1.2 Partícula carregada em um campo magnético intenso não-uniforme . . . . . . . . . 44

2.2 Aspectos gerais de teorias de campos não-comutativos _ . . . . . . . . . . . . . . 47

2.3 QED não-comutativa . . . . . . . . . . . . . . . . . . . . . . 49

2.4 Ação da QED não-comutativa . . . . . . . . . . . . . . . . . . . . . . . . . 49

2.4 .1 Regras de Feynman da NCQED . . . . . . . . . . . . . . . . . . 51

2.4 .2 Auto-energia do fóton $\ldots \ldots \ldots \ldots \ldots \ldots \ldots$ 
3 QED Não-Comutativa a Temperatura Finita $\quad 59$

3.1 Decomposição do tensor de auto-energia . . . . . . . . . . . . . . . . . . . . . . 59

3.2 Auto-energia do fóton (amplitude de 2-pontos) $\ldots \ldots \ldots \ldots$. . . . . . . . . . 61

3.2.1 Soma sobre as freqüências para a auto-energia do fóton . . . . . . . . . . . . 63

3.3 Blindagem dos campos elétrico e magnético . . . . . . . . . . . . . . . . . . . 65

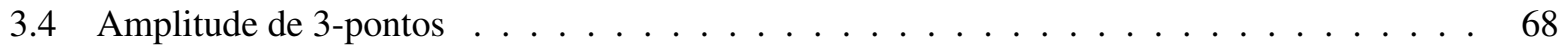

3.5 Amplitude de 4 -pontos $\ldots \ldots \ldots \ldots \ldots \ldots \ldots$

3.6 Identidades de Ward . . . . . . . . . . . . . . . . . . . . . . . . . . . 71

3.7 Ação efetiva . . . . . . . . . . . . . . . . . . . . . . . . . . . . 72

3.8 Método da amplitude de espalhamento frontal $\ldots \ldots \ldots \ldots \ldots$

3.9 Pressão de um gás de fótons não-comutativos $\ldots \ldots \ldots \ldots \ldots \ldots$

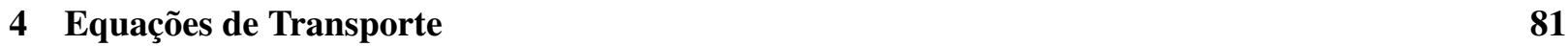

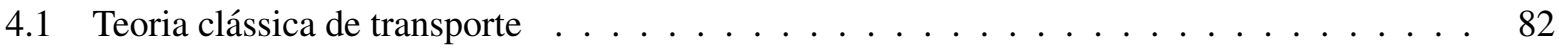

4.1 .1 Corrente de partículas $\ldots \ldots \ldots \ldots \ldots$

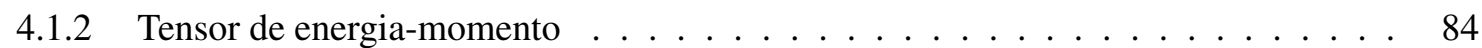

4.1.3 Equação de transporte para $f(\vec{x}, \vec{p}, t) \ldots \ldots \ldots \ldots$

4.2 Teoria quântica de transporte $\ldots \ldots \ldots \ldots \ldots \ldots \ldots$

4.2.1 Operador de Wigner não-relativístico ～. . . . . . . . . . . . . . . . . . . . . 87

4.2 .2 Operador de Wigner relativístico . . . . . . . . . . . . . . . . . . . . 88

4.2 .3 Operador de Wigner para férmions . . . . . . . . . . . . . . . . . . . . . . . . . 89

4.2.4 Operador de Wigner para campos de gauge . . . . . . . . . . . . . . . . . 90

5 Equações de Transporte na QED Não-Comutativa $\quad 93$

5.1 Tratamento clássico . . . . . . . . . . . . . . . . . . . . . . . . . . . . . . 94

5.1.1 Partículas carregadas - elétrons . . . . . . . . . . . . . . . . . 96

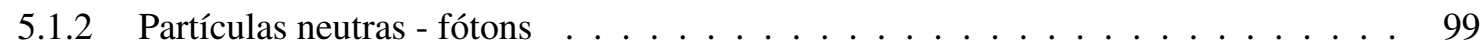

5.2 Tratamento quântico - O operador de Wigner para os fótons . . . . . . . . . . . . . . . . 100

5.3 Forma simplificada da função de Wigner para fótons . . . . . . . . . . . . . . . . 104

5.3.1 Cálculo de $\mathcal{F}(x, k) \ldots \ldots \ldots \ldots \ldots \ldots$

$5.3 .2 \quad$ Amplitude de 2 -pontos . . . . . . . . . . . . . . . . . . . . . 108

5.3 .3 Amplitude de 3 -pontos . . . . . . . . . . . . . . . . . . . 110

5.3 .4 Força para partículas neutras . . . . . . . . . . . . . . . . . 110

5.3 .5 Pressão na NCQED . . . . . . . . . . . . . . . . . . . . . . . . . . . 111

$\begin{array}{ll}\text { Conclusão } & 115\end{array}$

$\begin{array}{ll}\text { Apêndice } & 118\end{array}$ 
A.1 Revisão de conceitos de mecânica . . . . . . . . . . . . . . . . . . . . . . . . . . . . 119

B

B.1 Algumas propriedades do teorema dos resíduos . . . . . . . . . . . . . . . . . 120

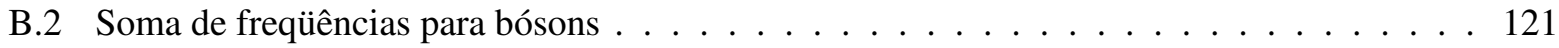

B.3 Soma de frequiências para férmions . . . . . . . . . . . . . . . . . . 125

C

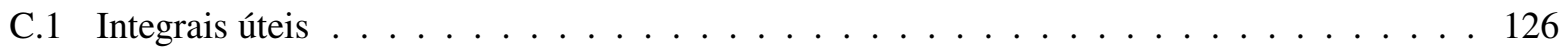

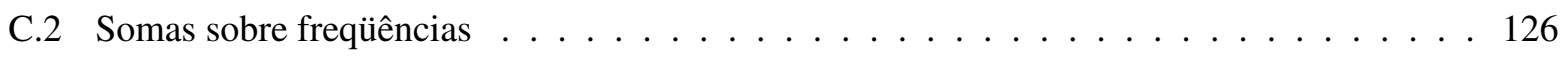

C.3 Expansões em série e identidades trigonométricas . . . . . . . . . . . . . . . . . . 127

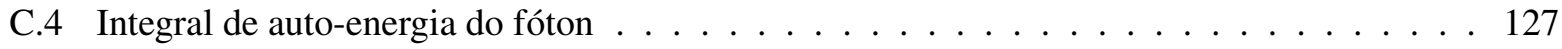

D

128

D.1 Método da soma sobre freqüências para a auto-energia do fóton . . . . . . . . . . . . 128

D.2 Integrais da auto-energia do fóton $\ldots \ldots \ldots \ldots \ldots \ldots \ldots \ldots \ldots$

D.3 Método da amplitude de espalhamento frontal $\ldots \ldots \ldots \ldots \ldots$

E 136

E.1 Amplitude de 3-pontos . . . . . . . . . . . . . . . . . 136

E.2 Amplitude de 4-pontos . . . . . . . . . . . . . . . . . . . 137

F 139

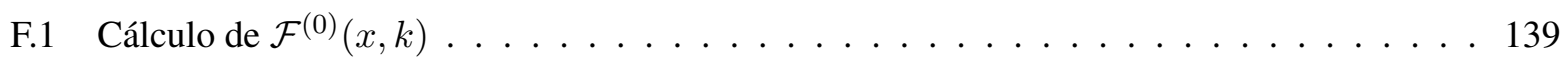

F.2 Cálculo de $\mathcal{F}^{(1)}(x, k) \ldots \ldots \ldots \ldots \ldots \ldots \ldots \ldots \ldots$

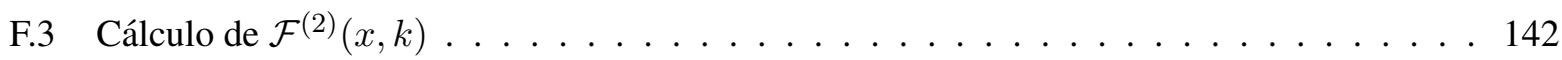

G $\quad 144$

G.1 Quantização da QED não-comutativa em um campo de fundo . . . . . . . . . . . . . . . . 144

$\begin{array}{ll}\text { Referências bibliográficas } & 149\end{array}$ 


\section{Introdução}

A teoria quântica de campos é um conjunto de idéias e de ferramentas que combina três das mais importantes áreas da física moderna, são elas a teoria da relatividade, a mecânica quântica e o conceito de campos, análogo aos campos eletromagnéticos da teoria de Maxwell. Ela é a base da moderna física das partículas elementares, e fornece o ferramental necessário para entender a física nuclear, a física atômica, a física da matéria condensada e a astrofísica, além de fazer uma ponte entre a matemática e muitas áreas da física. A necessidade de uma nova física para tratar de sistemas em escalas de distâncias muito pequenas e escalas de energias muito altas se revelou necessária quando a aplicação da mecânica quântica de Schrödinger não foi capaz de explicar alguns efeitos conhecidos, como a produção de novas partículas em colisões em aceleradores, ou a discrepância entre o valor medido experimentalmente e o calculado teoricamente para o momento magnético anômalo do elétron, ou ainda a pequena diferença de energia que existe entre os estados $2 S_{1 / 2}$ e $2 P_{1 / 2}$ do átomo de hidrogênio, efeito conhecido como "Lamb shift". Estas e outras dificuldades levaram Schwinger, Feynman, Dirac e outros à formulação da Eletrodinâmica Quântica, ou QED (Quantum Electrodynamics), considerada uma das melhores e mais fundamentais teorias de toda a física e nascida da teoria quântica de campos. É a teoria que explica corretamente as interações entre elétrons e fótons, formulada por meio de um conjunto simples de equações: as equações de Maxwell da eletrodinâmica e a equação de Dirac para os elétrons. As soluções que derivam desta teoria dão previsões detalhadas de fenômenos eletromagnéticos desde distâncias macroscópicas até regiões centenas de vezes menor que o tamanho do núcleo atômico.

A Eletrodinâmica Quântica apareceu alguns anos depois da mecânica quântica de Schrödinger. A idéia original de Planck da quantização da energia foi estendida e aplicada ao eletromagnetismo, e os "quantas" correspondentes ao campo eletromagnético foram identificados como sendo os fótons. Mais uma vez a dualidade onda-partícula se manifestava, já que o campo eletromagnético clássico é descrito por uma onda, mas a hipótese de quantização associa a este mesmo campo uma partícula, o fóton, que se propaga também como uma partícula. A esse processo deu-se o nome de segunda quantização, pois agora os campos associados às partículas também satisfazem a uma relação de comutação análoga àquela satisfeita pelas coordenadas e momentos conjugados da mecânica quântica usual. O desenvolvimento da QED se mostrou tão eficiente que os físicos formularam uma teoria nas mesmas bases para descrever as interações nucleares fracas, responsável pelo decaimento $\beta$ por exemplo, sendo as partículas mediadoras os chamados bósons massivos $W^{+}, W^{-}$e $Z^{0}$. Um modelo semelhante também foi aplicado para explicar as interações nucleares fortes, que são as interações entre os quarks, constituintes dos prótons e nêutrons, cujo campo 
análogo ao do fóton seria o glúon, de maneira que nasceu então a Cromodinâmica Quântica, ou QCD (Quantum Cromodynamics). Assim como os fótons são os quantas que intermediam as interações eletromagnéticas entre elétrons por exemplo, os glúons são os quantas que intermediam as interações nucleares fortes entre os quarks. Por último fez-se a tentativa de quantizar a gravitação, em um esforço para juntar a mecânica quântica com a teoria da relatividade geral de Einstein, sendo o gráviton a partícula mediadora das interações gravitacionais. Mas esta é uma área que ainda está em desenvolvimento, a teoria de cordas é uma das possíveis candidatas, mas existem inúmeras dificuldades que ainda precisam ser contornadas e portanto trata-se de um problema ainda em aberto.

Vamos retornar então à QED, a teoria que estamos interessados aqui. Um dos principais métodos de cálculo, que derivaram do desenvolvimento da teoria quântica de campos e em particular da QED, são os conhecidos diagramas de Feynman. Este método se mostrou bastante útil no tratamento de processos de espalhamento e de produção de partículas, já que uma análise exata destes processos não é factível na prática. Então o que se propõe é tratá-los perturbativamente ordem a ordem em algum parâmetro do problema, geralmente a constante de acoplamento, de maneira que os cálculos explícitos possam ser feitos ordem a ordem, fornecendo correções cada vez mais precisas. Para sermos mais claro, imagine um processo simples que envolva elétrons e fótons, como o espalhamento Møller, que nada mais é que o efeito clássico de repulsão coulombiana entre duas partículas de mesma carga, por exemplo dois elétrons se aproximando, interagindo de alguma maneira e em seguida se distanciando. Do ponto de vista da QED podemos desenhar o que acontece neste processo por um diagrama de Feynman, ele é dado pela figura abaixo, que deve ser "lido" da esquerda para a direita, indicando que dois elétrons se aproximam, interagem entre si trocando um fóton, e em seguida continuam seus caminhos. Sabemos que a um tal espalhamento está associada

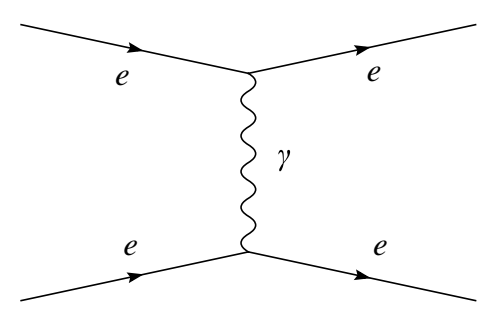

uma amplitude, que está diretamente relacionada à seção de choque do processo em questão. Portanto, calcular um diagrama de Feynman do tipo ilustrado nesta figura significa calcular com qual probabilidade este espalhamento deve ocorrer. Mas temos de dizer aqui que este cálculo é apenas uma aproximação do que acontece realmente. De fato, este diagrama representando o espalhamento Møller não é tudo o que acontece. Apenas em primeira aproximação o resultado está correto, e uma característica notável desta formulação por meio de diagramas de Feynman é que podemos construir, ordem a ordem, qual é o processo real que de fato acontece, e obter uma expressão cada vez mais precisa para a amplitude envolvida no espalhamento, ou seja, temos como tornar o cálculo da seção de choque envolvida cada vez mais preciso.

Vamos ser mais claros no que estamos querendo dizer. $\mathrm{O}$ fóton que está intermediando a interação entre os elétrons pode, segundo a formulação da QED, sofrer uma outra interação antes de fazer a intermediação 
entre os dois elétrons, o que poderia mudar um pouco o valor da amplitude final deste processo. De fato isto acontece, e outro diagrama que pode também contribuir para a amplitude final é, por exemplo, representado abaixo. Ele indica que o fóton trocado pelos elétrons pode, em algum instante, formar um

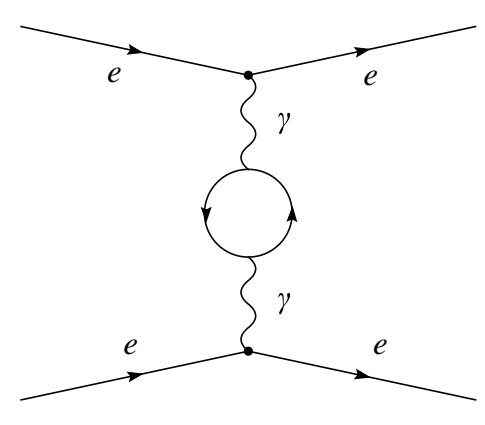

par elétron-pósitron, que se aniquilam logo em seguida formando novamente um fóton, que só depois vai transferir a interação. Dizemos que o propagador do fóton foi modificado. Diagramas deste tipo são chamados diagramas de 1-laço, ou 1-loop, como é mais comum na literatura. Portanto, a amplitude final a ser considerada deve levar em conta também este possível efeito, sendo a contribuição para a seção de choque final uma soma sobre todas as possibilidades. Na verdade existem muitos outros diagramas que podem contribuir para este espalhamento, mas felizmente eles dão contribuições cada vez menores, de maneira que em primeira aproximação podemos nos restringir ao cálculo de uns poucos diagramas. Este é o mérito dos diagramas de Feynman. Podemos obter, se quisermos, correções cada vez mais precisas para a amplitude em questão analisando apenas os possíveis diagramas que contribuem para o espalhamento. Não é preciso dizer que os cálculos se tornam cada vez mais complicados, mas o fato é que temos um método sistemático de fazer cálculos. Portanto, as primeiras correções a um processo direto vêm de diagramas do tipo 1-loop, como o representado acima, e serão diagramas desse tipo que estaremos interessados aqui.

Agora vamos adicionar mais um ingrediente a esta nossa brevíssima introdução a teoria quântica de campos. Imagine que um processo do tipo representado nos diagramas anteriores esteja ocorrendo imerso em um reservatório térmico de temperatura $T$, banhando todo o sistema. Será que a temperatura tem alguma influência no cálculo das amplitudes? É de se esperar que sim, afinal de contas uma fonte externa de calor significa uma fonte de energia, o que pode perfeitamente influenciar processos de espalhamento e produção de partículas. Sabemos que as partículas estão interagindo entre si, processos como os descritos anteriormente estão ocorrendo a todo momento, entre milhares de outros que são também representados por diagramas de Feynman, e todos eles ocorrem imersos em um banho térmico. Portanto, nada mais natural que perguntarmos: o que muda com os cálculos da teoria quântica de campos usual se o sistema estiver sob efeito de altas temperaturas e densidades? Foi então que nasceu a teoria quântica de campos a temperatura finita.

São diversos os sistemas onde efeitos de temperatura e densidade ocorrem. O interior de estrelas por exemplo é um bom exemplo. Qual o mecanismo que rege o comportamento da matéria nos núcleos de estrelas de nêutrons ou de anãs brancas? De que forma o limite de Chandrasekhar, que determina se 
uma estrela vai ou não colapsar para um buraco negro, pode ser influenciado por efeitos de temperatura e densidade? Além desses interesses astrofísicos, uma questão fundamental na física atual é entender o comportamento de plasmas da QCD a altíssimas temperaturas e densidades. Acredita-se que acima de uma certa temperatura crítica possa existir uma fase da matéria onde quarks e glúons possam coexistir de forma independente, ao contrário do que ocorre na matéria hadrônica nuclear, onde eles não são encontrados separadamente. Este possível estado da matéria ficou conhecido como "plasma de quarks e glúons", que pode ter existido naturalmente no universo primordial. Cálculos de QCD na rede indicam que de fato deve ocorrer uma transição de fases com a matéria nuclear a altíssimas temperaturas e/ou densidades. O diagrama de fases Temperatura $(T) \times$ Potencial químico $(\mu)$ da figura abaixo dá uma visão mais clara desse processo. A baixas temperaturas e densidades da ordem da densidade nuclear, quarks e glúons estão

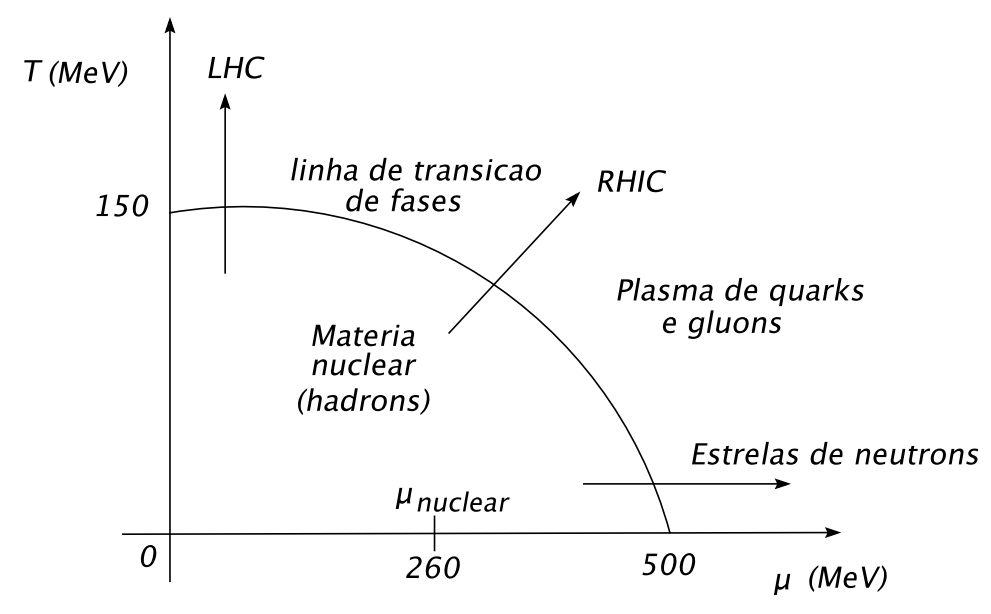

confinados formando hádrons. Não existem quarks ou glúons livres se propagando no vácuo. Mas esta situação pode mudar se a temperatura subir acima de um valor crítico da ordem de $150 \mathrm{MeV}$. Nesta situação podemos ter um gás de quarks e glúons quase livres, o chamado plasma de quarks e glúons. Esta mesma situação pode ocorrer em temperaturas muito baixas e densidades muito altas, da ordem de cinco vezes a densidade nuclear (que corresponde a cerca de duas vezes o potencial químico nuclear), de forma que também pode haver esta transição de fases. Cálculos de QCD na rede confirmam o valor da temperatura crítica acima para potencial químico nulo, mas ainda não existem valores confiáveis para o caso inverso, de temperatura nula e $\mu$ grande. A figura indica alguns "laboratórios" onde estas situações físicas podem ocorrer.

São vários os métodos desenvolvidos para tratar sistemas quânticos a temperatura finita. O mais comum é o que faz uma ligação direta entre a termodinâmica estatística e a teoria de campos, que ficou conhecido como formalismo do tempo imaginário, em que a parte temporal da ação de um sistema da teoria quântica de campos usual é estendida para o eixo imaginário. Como resultado temos uma estreita analogia entre o funcional gerador das funções de Green da teoria de campos com a função de partição da termodinâmica estatística, da qual derivam as relações de entropia, energia livre, pressão, etc, para um sistema em equilíbrio térmico. Desta forma o método dos diagramas de Feynman continua sendo válido, 
apenas com algumas modificações.

Um outro tratamento que leva em conta diretamente a temperatura de um sistema foi desenvolvido por Boltzmann no final do século XIX. Um sistema perto do equilíbrio pode ser descrito por uma função distribuição que caracteriza várias propriedades do sistema. Devido a algum efeito externo, a situação de equilíbrio não é mais satisfeita e isto se reflete em uma mudança na função distribuição do sistema, que agora deve satisfazer a uma equação dependente das equações de movimento clássicas do sistema. Esta equação é a chamada equação de transporte, que quando tratada perturbativamente é um conjunto de equações. Portanto, obtidas as correções para a função distribuição estamos obtendo as características do sistema fora do equilíbrio. À primeira vista este é um método totalmente clássico, mas em alguns limites, por exemplo quando a temperatura do sistema é muito alta, podemos aplicá-lo para tratar de problemas da teoria quântica de campos. Existe uma segunda formulação das equações de transporte, baseada em uma construção quântica, que foi desenvolvida por Wigner e é mais apropriada para tratar sistemas em teoria de campos. Tanto o método tradicional do formalismo do tempo imaginário quanto o método das equações de transporte clássica e quântica serão discutidos com mais detalhes nos capítulos que seguem. Vamos agora adicionar mais um ingrediente final à nossa teoria quântica de campos.

Sabemos que uma característica importante e marcante da mecânica quântica e que tem reflexos em muitas situações físicas importantes é a quantização da energia de sistemas microscópicos. Isto decorre do fato básico de que na mecânica quântica os operadores que representam as coordenadas e momentos conjugados de uma partícula não comutam, ou seja, $\left[\hat{x}, \hat{p}_{x}\right]=i \hbar$. A teoria quântica de campos é construída nesta mesma base, sendo a quantização imposta aos operadores de campo (segunda quantização), e isso tem implicações profundas em toda a física nuclear e atômica por exemplo. Agora imagine que, além da não-comutatividade entre coordenadas e momentos, impomos também que as próprias coordenadas não comutem entre si, ou seja, $[x, y] \neq 0$ por exemplo. Você poderia se perguntar agora que implicações isto teria para a teoria quântica de campos tal como a conhecemos até agora e qual a motivação para se estudar sistemas que satisfaçam esta relação de comutação estranha. E a resposta é que são muitas as motivações assim como as modificações da teoria usual. Recentemente tem havido um interesse muito grande por teorias quânticas de campos definidas em espaços não-comutativos, em parte pelos novos desenvolvimentos da teoria das cordas, que prevê que em determinado limite onde um campo magnético intenso está presente a não-comutatividade das coordenadas aparece naturalmente.

Temos portanto todas as ferramentas necessárias para dizer a que se propõe nosso trabalho aqui. Queremos fazer cálculos de diagramas de 1-loop da eletrodinâmica quântica a temperatura finita definida em um espaço não-comutativo. Primeiro vamos utilizar o método tradicional dos diagramas de Feynman, e depois vamos mostrar que, a altas temperaturas, os mesmos cálculos podem ser feitos usando-se um método baseado nas equações de transporte de Boltzmann, portanto um método clássico. Isto acontece porque termos de ordem dominante (clássicos) aparecem também dos diagramas de 1-loop a altas temperaturas.

No primeiro capítulo vamos rever algumas características da eletrodinâmica quântica, tanto a temperatura nula quanto a temperatura finita, a fim de fazermos uma conexão entre os resultados existentes para a QED usual e os resultados novos que vamos apresentar nos capítulos seguintes. No segundo capítulo 
discutimos alguns aspectos da teoria de campos em espaços não-comutativos, algumas motivações e um exemplo quase clássico, em que uma partícula carregada na presença de um campo magnético uniforme desenvolve naturalmente a não-comutatividade das coordenadas. Um tratamento da generalização para campos magnéticos facamente não-uniformes também é apresentada, o que resultou na publicação dos artigos [72, 73]. Também vamos discutir como as regras de Feynman se modificam para a QED nãocomutativa e a expressão para alguns diagramas de 1-loop. No terceiro capítulo desenvolvemos a primeira parte de nosso trabalho, aplicando as técnicas dos dois primeiros capítulos especificamente para a QED em espaços não-comutativos e a temperatura finita. Os principais resultados apresentandos neste capítulo foram publicados na referência [70]. No quarto capítulo fazemos uma breve exposição geral do método das equações de transporte de Boltzmann, apresentamos alguns modelos já estudados anteriormente por outros e no quinto capítulo aplicamos o modelo ao nosso caso da QED não-comutativa, mostrando que os mesmos resultados do terceiro capítulo podem ser obtidos. Para isso estudamos a quantização do campo de gauge quântico na presença de um campo, resultado apresentado no Apêndice G e publicado na referência [71].

Para não tornar o texto cansativo com tecnicalidades matemáticas, algum material foi colocado no apêndice, para ser consultado quando necessário sem perder a lógica do texto principal.

\section{Notações e convenções}

Ao longo do texto usaremos as seguintes notações e convenções:

Métrica de Minkovski $\eta^{\mu \nu}=\operatorname{diag}(1,-1,-1,-1)$.

Exceto no capítulo 1, adotaremos $\hbar=c=k_{B}=1$.

Neste sistema, $1 \mathrm{GeV}^{-1} \sim 10^{-14} \mathrm{~cm}$. $1 \mathrm{GeV} \sim 10^{13} \mathrm{~K}$.

Exceto no capítulo $1, k$ se refere ao momento interno ao loop e $p$ ao momento externo. 


\section{Capítulo 1}

\section{Alguns Aspectos da Eletrodinâmica Quântica}

Neste primeiro capítulo vamos apresentar alguns resultados básicos da eletrodinâmica quântica que serão importantes para o entendimento do contexto em que se insere o presente projeto. O objetivo é mostrar onde nossos cálculos serão relevantes, quais as motivações físicas, enfim, fazer uma conexão com o mundo físico dos nossos resultados. Na primeira seção derivamos alguns resultados da eletrodinâmica quântica usual, ou a temperatura nula, para entender onde exatamente os cálculos a temperatura finita serão relevantes. Alguns aspectos da QED a temperatura finita são apresentados na segunda seção. Nada do que está apresentado neste capítulo é novo, queremos apenas fornecer informações para que possamos ter com o que comparar quando nossos cálculos forem apresentados nos capítulos seguintes. Além disso, detalhes de cálculos complexos não são feitos, mas referências onde eles podem ser encontrados são dadas ao longo do texto.

\subsection{QED a temperatura nula}

Já foi dito na introdução que a eletrodinâmica quântica é a teoria física mais completa e precisa que existe até o momento, sendo capaz de reproduzir valores experimentais com altíssimo grau de precisão. As chamadas correções radiativas para um dado processo aproximam cada vez mais o valor teórico do obtido experimentalmente, embora os cálculos fiquem cada vez mais complicados de serem feitos. Mas antes de nos embrenharmos por cálculos longos e complicados que têm muito pouco a ver com a física do nosso dia-a-dia, vamos tentar situar num contexto mais amplo qual é exatamente o nosso objetivo neste trabalho. Para isto vamos retomar a teoria de campos usual, ou teoria de campos a temperatura nula, com o objetivo de mostrar exatamente onde entrará o tratamento a temperatura finita. 


\subsubsection{Lagrangeana e regras de Feynman da QED}

Vamos apresentar brevemente aqui algumas das ferramentas matemáticas necessárias para se fazerem cálculos na eletrodinâmica quântica. São as chamadas regras de Feynman ${ }^{1}$, cujos objetos mais importantes são os "propagadores" e "vértices". Eles derivam diretamente da lagrangeana do sistema em questão, no nosso caso a lagrangeana da eletrodinâmica quântica, que é composta de campos fermiônicos $\psi(x)$ e $\bar{\psi}(x)$ de spin $\frac{1}{2}$, necessários para descrever elétrons, pósitrons, múons, etc., e de campos que representam o potencial vetor eletromagnético, $A^{\mu}(x)$, de spin 1, para descrever os fótons que são responsáveis pela interação eletromagnética.

1 - Propagadores - A lagrangeana da QED é dada por:

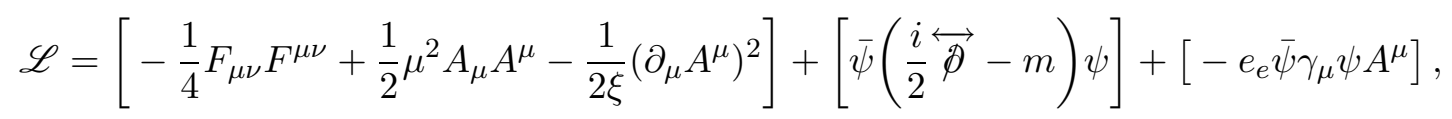

onde $F^{\mu \nu}=\left(\partial^{\mu} A^{\nu}-\partial^{\nu} A^{\mu}\right)$ é o tensor eletromagnético. A carga do elétron é representada por $e_{e}$, e esta notação ficará mais clara adiante. No primeiro colchetes, o termo com $\mu^{2}$ representa uma massa para o fóton, em alguns cálculos é conveniente tomar a massa do fóton não-nula e só no final fazê-la tender a zero. Para nossa discussão isto não importa, de modo que podemos tomar $\mu^{2}=0$. O termo contendo $1 / \xi$ é chamado termo de fixação de calibre, ou fixação de gauge, mas os resultados finais não devem depender de $\xi$, o que reflete uma "invariância de gauge" da teoria. Dois limites importantes de $\xi$ são $\xi \rightarrow 1$ (gauge de Feynman) e $\xi \rightarrow 0$ (gauge de Landau). Observe que todos os fatores dentro do primeiro colchetes são proporcionais a $A A$. Isto indica a possibilidade de uma partícula, no caso um fóton, se "propagar" de um ponto ao outro, sendo criada por um dos $A$ 's e destruída pelo outro ${ }^{2}$. Isto dará uma contribuição às regras de Feynman no espaço dos momentos com a seguinte regra:

$$
\text { น น }
$$

O termo seguinte da lagrangeana, formado por $\bar{\psi}$ e $\psi$, representa a possibilidade de um férmion, um elétron por exemplo, se propagar de um ponto ao outro, e a correspondente regra de Feynman é:

$$
\stackrel{p}{\longrightarrow}: \quad S_{0} \equiv \frac{i}{\not p-m c}=\frac{i(\not p+m c)}{p^{2}-m^{2} c^{2}}
$$

E finalmente o último termo, proporcional a $\bar{\psi} \psi A$, é o responsável pela interação entre o fóton e o elétron. Ele nos diz que estas partículas podem estar "ligadas" em algum momento do espaço-tempo. Outra maneira de entender isso é que o campo eletromagnético produzido pelo elétron é transmitido pelo espaço através de fótons. A correspondente regra de Feynman é chamada fator de vértice, e é representada

\footnotetext{
${ }^{1}$ Não vamos apresentar a dedução completa das regras para propagadores e vértices, isto está feito em vários livros texto de teoria de campos. Queremos apenas dar uma idéia formal de como elas são derivadas. Boas referências neste assunto são $[1,2,3,4,5,6]$

${ }^{2}$ Esta é apenas uma descrição formal. Os campos que compõem a lagrangeana são operadores, e estes operadores agindo no espaço de Hilbert é que são interpretados como operadores de criação e de aniquilação de partículas.
} 
por:

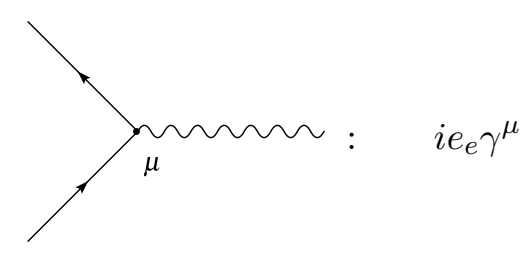

A constante $e_{e}$ é a constante de acoplamento (ou intensidade) da interação, e no caso da eletrodinâmica quântica é a carga do elétron.

Estas foram as regras dos propagadores e vértice. Agora vamos apresentar brevemente as regras para as linhas externas:

2 - A cada elétron (ou outro férmion qualquer) com momento $p$ entrando em um vértice, devemos atribuir um espinor $u^{(s)}(p)$, e para um elétron saindo, um espinor $\bar{u}^{(s)}(p)$, onde $s=1,2$ indica o spin da partícula. Estes espinores satisfazem as equações de Dirac no espaço dos momentos

$$
\left(\gamma^{\mu} p_{\mu}-m c\right) u^{(s)}(p)=0 \quad \bar{u}^{(s)}(p)\left(\gamma^{\mu} p_{\mu}-m c\right)=0
$$

e são ortogonais $\bar{u}^{(1)} u^{(2)}=0$ e normalizados $\bar{u} u=2 m c$.

3 - Para um pósitron (ou antiférmion) entrando, atribuímos um espinor $v^{(s)}(p)$, e para um pósitron saindo, um espinor $\bar{v}^{(s)}(p)$. Eles satisfazem as equações

$$
\left(\gamma^{\mu} p_{\mu}+m c\right) v^{(s)}(p)=0 \quad \bar{v}^{(s)}(p)\left(\gamma^{\mu} p_{\mu}+m c\right)=0
$$

e são ortogonais $\bar{v}^{(1)} v^{(2)}=0$ e normalizados $\bar{v} v=-2 m c$.

4 - Para um fóton entrando, escrevemos $\epsilon^{\mu}$, e para um fóton saindo $\epsilon^{\mu *}$, que correspondem às diferentes polarizações do fóton.

5 - Conservação de energia e momento - Para cada vértice, incluir uma função delta da forma

$$
(2 \pi)^{4} \delta^{4}\left(k_{1}+k_{2}+k_{3}\right) .
$$

6 - Integração sobre momentos internos - Para cada momento interno $q$, incluir um termo

$$
\int \frac{\mathrm{d}^{4} q}{(2 \pi)^{4}}
$$

e integrar.

7 - Para cada loop fermiônico incluir um fator $(-1)$.

8 - No resultado final vai restar um fator

$$
(2 \pi)^{4} \delta^{4}\left(p_{1}+p_{2}+\cdots-p_{n}\right),
$$

correspondente à conservação de energia e momento. Cancelando este termo, o que resta é a amplitude 
$-i \mathcal{M}$.

Estas são as peças fundamentais para construirmos diagramas de Feynman, e acredite, muitos diagramas diferentes podem ser construídos utilizando somente estas regras, como veremos mais à frente.

\subsubsection{Seção de choque}

Uma das quantidades físicas mais importantes e que podem ser obtidas experimentalmente no laboratório é a seção de choque de uma reação, que nada mais é que a probabilidade de que uma certa reação ou processo de espalhamento possa ocorrer. Considere por exemplo um processo em que uma partícula $A$ se choca com uma partícula $B$ dando origem a duas outras partículas $C$ e $D$,

$$
A+B \rightarrow C+D \text {. }
$$

Este processo simples pode muito bem descrever várias situações físicas de interesse, por exemplo, os seguintes processos:

$$
\begin{array}{cc}
e^{-}+e^{-} \rightarrow e^{-}+e^{-} & \text {espalhamento elétron-elétron } \\
\gamma+e^{-} \rightarrow \gamma+e^{-} & \text {espalhamento Compton } \\
e^{-}+p^{+} \rightarrow e^{-}+p^{+} & \text {espalhamento elétron-próton } \\
e^{-}+e^{+} \rightarrow \gamma+\gamma & \text { aniquilação de pares } \\
e^{-}+e^{+} \rightarrow \mu^{-}+\mu^{+} & \text {produção de múons }
\end{array}
$$

dentre outros. Para qualquer um deles o esquema (1.10) é válido, e no referencial do centro de massa do sistema a seção de choque diferencial pode ser escrita como

$$
\frac{\mathrm{d} \sigma}{\mathrm{d} \Omega}=F\left(\frac{\hbar c}{8 \pi}\right)^{2} \frac{|\mathcal{M}|^{2}}{\left(E_{A}+E_{B}\right)^{2}} \frac{\left|\vec{p}_{f}\right|}{\left|\vec{p}_{i}\right|},
$$

onde $\vec{p}_{f}=\left|\vec{p}_{C}\right|=\left|\vec{p}_{D}\right|$ é o momento final das partículas, $\vec{p}_{i}=\left|\vec{p}_{A}\right|=\left|\vec{p}_{B}\right|$ é o momento inicial, $E_{A}$ e $E_{B}$ são as energias relativísticas $E_{j}=\sqrt{m_{j}^{2} c^{4}+\vec{p}_{j}^{2} c^{2}}$ das partículas e $p_{j}=\left(E_{j} / c, \vec{p}_{j}\right)$ é o quadrivetor momento, com $j=A, B$. O fator $F$ é um produto de fatores estatísticos que leva em conta as partículas no estado final serem iguais ou não. Fica faltando dizer o que é $\mathcal{M}$. Esta quantidade recebe o nome de amplitude de transição ou simplesmente amplitude, e seu cálculo depende basicamente das regras de Feynman indicadas anteriormente. Qualquer um dos processos descritos anteriormente pode ocorrer de várias maneiras, com estágios intermediários, e o que diz qual é o processo mais provável é a amplitude $\mathcal{M}$, sendo o processo completo formado pela soma de todas as amplitudes. Para ficar mais claro o que estamos querendo dizer, vamos considerar dois exemplos a seguir, a produção de múons pela aniquilação entre um elétron e um pósitron e o espalhamento pelo potencial coulombiano. 


\subsubsection{Produção de múons}

O múon é um lépton com exatamente todas as mesmas propriedades do elétron, mesma carga, mesmo spin, exceto sua massa, que é $m_{\mu} \approx 200 m_{e}$. A reação

$$
e^{-}+e^{+} \rightarrow \mu^{-}+\mu^{+}
$$

ocorre em grande quantidade dentro de aceleradores, como no LEP, no SLAC, no RHIC, e o valor teórico de sua seção de choque é muito fácil de calcular, podendo ser confrontada com os valores obtidos naqueles laboratórios.

Uma forma diagramática simples de representar esta reação é a dada na Figura 1.1. "Lendo" da es-

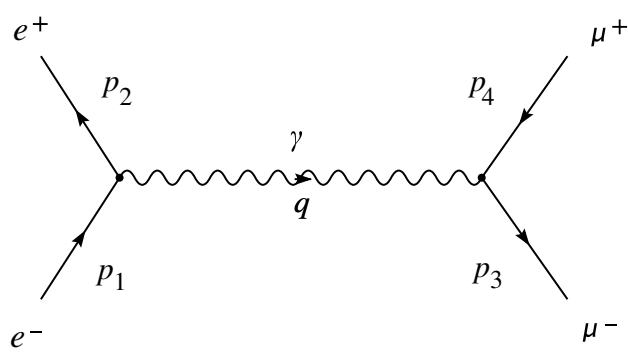

Figura 1.1: Produção de múons através da colisão elétron-pósitron.

querda para a direita, este gráfico diz o seguinte. Um elétron e um pósitron se aniquilam, dando origem a um fóton virtual $\gamma$ que logo se desintegra, formando em seguida um par múon-antimúon. Este diagrama dará origem a uma amplitude $\mathcal{M}_{1}$, que de acordo com as regras de Feynman (1.2)-(1.9), será dada por

$$
\mathcal{M}_{1}=-e_{e}^{2}\left[\bar{u}\left(p_{3}\right) \gamma^{\mu} u\left(p_{4}\right)\right] \frac{\eta_{\mu \nu}}{q^{2}}\left[\bar{u}\left(p_{2}\right) \gamma^{\nu} u\left(p_{1}\right)\right]
$$

onde $q=p_{1}-p_{2}$. Embora tenha uma aparência complicada, o cálculo de $\mathcal{M}_{1}$ pode ser feito sem complicações, e o resultado é simplesmente um número, que deve ser colocado na equação (1.11) e a seção de choque diferencial pode ser calculada.

Mas esta não é toda a história. Como dissemos anteriormente, outros diagramas também podem contribuir para o processo com alguma amplitude, e de fato é isto o que realmente acontece. O diagrama da Figura 1.2 mostra uma outra maneira possível de ocorrer a produção de múons. O elétron e o pósitron se aniquilam formando um fóton virtual, este fóton se desintegra em um par elétron-pósitron que em seguida se aniquilam formando novamente um fóton virtual, e finalmente este fóton se desintegra em um par de múons. Esta é uma possibilidade ditada pelas regras da QED e que de fato acontece, mesmo tendo uma amplitude $\mathcal{M}_{2}$ com ordem de grandeza bem menor. Trata-se de um diagrama de 1-laço, ou 1-loop, conhecido como "polarização do vácuo", tendo a propriedade de modificar a carga do elétron $e_{e}$, como ficará 


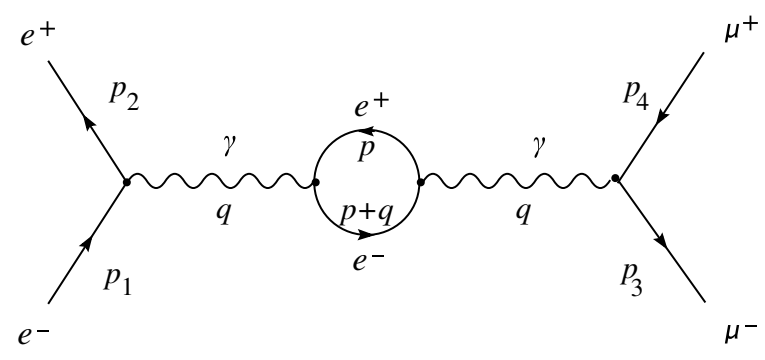

Figura 1.2: Diagrama de 1-loop que contribui para o processo de produção de múons.

mais claro adiante. A expressão matemática para esta amplitude é, usando as regras de Feynman,

$$
\mathcal{M}_{2}=\frac{i e_{e}^{2}}{q^{4}}\left[\bar{u}\left(p_{3}\right) \gamma^{\mu} u\left(p_{4}\right)\right]\left\{-e_{e}^{2} \int \frac{\mathrm{d}^{4} p}{(2 \pi)^{4}} \frac{\operatorname{Tr}\left[\gamma_{\mu}(\not p+\not d+m c) \gamma_{\nu}(\not p+m c)\right]}{\left((p+q)^{2}-m^{2} c^{2}\right)\left(p^{2}-m^{2} c^{2}\right)}\right\}\left[\bar{u}\left(p_{2}\right) \gamma^{\nu} u\left(p_{1}\right)\right] \text {. }
$$

Note que esta contribuição é de ordem $e_{e}^{4}$, portanto muito menor que a contribuição de $\mathcal{M}_{1}$, que é de ordem $e_{e}^{2}$. O termo entre chaves recebe um nome especial, é a “auto-energia do fóton”, $\Pi_{\mu \nu}(q)$,

$$
\Pi_{\mu \nu}(q)=-e_{e}^{2} \int \frac{\mathrm{d}^{4} p}{(2 \pi)^{4}} \frac{\operatorname{Tr}\left[\gamma_{\mu}(\not p+\not p+m c) \gamma_{\nu}(\not p+m c)\right]}{\left((p+q)^{2}-m^{2} c^{2}\right)\left(p^{2}-m^{2} c^{2}\right)} .
$$

Note que esta expressão é exatamente aquela que obtemos se aplicarmos as regras de Feynman somente ao loop da Figura 1.3,

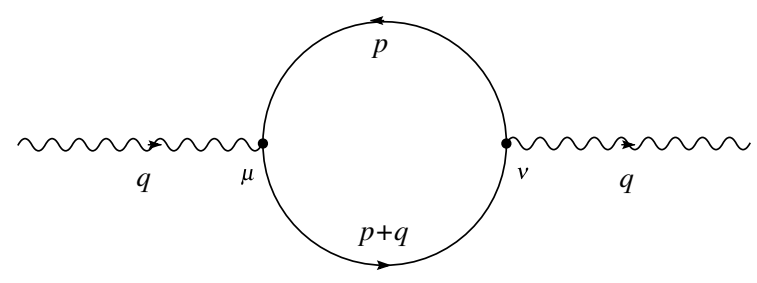

Figura 1.3: Diagrama de auto-energia do fóton.

Comparando $\mathcal{M}_{1}$ e $\mathcal{M}_{2}$ percebemos o que aconteceu. O propagador do fóton, que era simplesmente $\eta_{\mu \nu} / q^{2}$, fica modificado para

$$
\frac{\eta_{\mu \nu}}{q^{2}} \rightarrow \frac{\eta_{\mu \nu}}{q^{2}}-\frac{i}{q^{4}} \Pi_{\mu \nu}
$$

se levarmos em conta também a contribuição de $\mathcal{M}_{2}$. Para fazermos a integral em (1.14) devemos desenvolver o traço no numerador, e não é difícil mostrar que $\Pi_{\mu \nu}$ pode ser escrito da seguinte forma:

$$
\Pi_{\mu \nu}(q)=-i \eta_{\mu \nu} q^{2} I\left(q^{2}\right)+q_{\mu} q_{\nu} J\left(q^{2}\right)
$$


O segundo termo é nulo quando inserido dentro de $\mathcal{M}_{2}$ e feitas as contrações com $\gamma^{\mu}$ e $\gamma^{\nu}$. Portanto resta apenas fazer a integração que está em $I\left(q^{2}\right)$. Mas infelizmente esta integral é divergente quando $|p| \rightarrow \infty$. Basta olhar para a expressão (1.14). O denominador é de ordem $p^{4}$, enquanto que o numerador ${ }^{3}$ é de ordem $p^{5}$, portanto a integral é quadraticamente divergente. Para contornarmos este problema devemos adotar algum procedimento de regularização, que permite isolar a parte divergente do cálculo a fim de entendermos melhor o resultado. Uma maneira é fazer a integração até um valor de corte $\Lambda^{2}$ que só depois de todos os cálculos terem sido feitos deve tender ao infinito. O resultado será ${ }^{4}$

$$
I\left(q^{2}\right)=\frac{e_{e}^{2}}{12 \pi^{2}}\left\{\int_{m^{2}}^{\Lambda^{2}} \frac{\mathrm{d} y}{y}-6 \int_{0}^{1} z(1-z) \ln \left(1-\frac{q^{2}}{m^{2} c^{2}} z(1-z)\right) \mathrm{d} z\right\},
$$

de maneira que a parte divergente está na primeira integral, que é $\ln \left(\Lambda^{2} / m^{2}\right)$. A segunda integral é finita e por isso não precisamos nos preocupar com ela, sendo do tipo

$$
f(x) \equiv 6 \int_{0}^{1} z(1-z) \ln (1+x z(1-z)) \mathrm{d} z
$$

O resultado desta integral e alguns de seus limites estão no Apêndice C.4. Portanto temos

$$
I\left(q^{2}\right)=\frac{e_{e}^{2}}{12 \pi^{2}}\left\{\ln \left(\frac{\Lambda^{2}}{m^{2}}\right)-f\left(\frac{-q^{2}}{m^{2} c^{2}}\right)\right\} .
$$

Desta forma, a amplitude total $\mathcal{M}=\mathcal{M}_{1}+\mathcal{M}_{2}$ para a produção de múons é dada por

$$
\mathcal{M}=-e_{e}^{2}\left[\bar{u}\left(p_{3}\right) \gamma^{\mu} u\left(p_{4}\right)\right] \frac{\eta_{\mu \nu}}{q^{2}}\left\{1-\frac{e_{e}^{2}}{12 \pi^{2}}\left[\ln \left(\frac{\Lambda^{2}}{m^{2}}\right)-f\left(\frac{-q^{2}}{m^{2} c^{2}}\right)\right]\right\}\left[\bar{u}\left(p_{2}\right) \gamma^{\nu} u\left(p_{1}\right)\right]
$$

Agora vamos ver como nos "livrar" do infinito que está contido no parâmetro $\Lambda^{2}$ quando ele tende ao infinito. $\mathrm{O}$ truque é redefinir a constante de acoplamento $e_{e}$ (que é a carga do elétron) da seguinte maneira

$$
e_{R} \equiv e_{e} \sqrt{1-\frac{e_{e}^{2}}{12 \pi^{2}} \ln \left(\frac{\Lambda^{2}}{m^{2}}\right)} \text {. }
$$

Reescrevendo (1.14) em termos desta nova constante de acoplamento, temos

$$
\mathcal{M}=-e_{R}^{2}\left[\bar{u}\left(p_{3}\right) \gamma^{\mu} u\left(p_{4}\right)\right] \frac{\eta_{\mu \nu}}{q^{2}}\left\{1+\frac{e_{R}^{2}}{12 \pi^{2}} f\left(\frac{-q^{2}}{m^{2} c^{2}}\right)\right\}\left[\bar{u}\left(p_{2}\right) \gamma^{\nu} u\left(p_{1}\right)\right] .
$$

Agora note o que aconteceu. A divergência está toda contida na constante de acoplamento (1.21) e o resultado que sobra é finito e muito bem definido. Portanto, se na lagrangeana original (1.1) introduzirmos

\footnotetext{
${ }^{3}$ Lembre-se que o elemento de volume em quatro dimensões é proporcional a $p^{3}$.

${ }^{4}$ Os detalhes destes cálculos podem ser encontrados em diversos livros texto de teoria de campos, por exemplo as referências
} $[4,7,8]$. 
$e_{R}$ no lugar de $e_{e}$, os cálculos já saem finitos. Este é o chamado processo de renormalização. A constante de acoplamento é redefinida, ou renormalizada, de forma a absorver a divergência, e os cálculos que seguem são finitos. Mas esta constante de acoplamento é a carga do elétron, que é muito bem conhecida e tem valor finito. No entanto, a carga que se mede no laboratório é a carga $e_{R}$, que já leva em consideração todas as correções devido a processos do tipo da Figura 1.2, e a carga real que é medida por exemplo na repulsão entre dois elétrons é na verdade uma diferença entre cargas, e não a carga exata do elétron, que é $e_{e}$, mas a esta não temos acesso direto. No exemplo seguinte do espalhamento coulombiano vamos discutir um pouco mais este aspecto.

\subsubsection{Espalhamento coulombiano}

A repulsão coulombiana entre dois elétrons ou a atração entre o elétron e o próton são conceitos básicos da física conhecidos já há alguns séculos. Mas do ponto de vista quântico os mecanismos desta interação entre as cargas só veio mesmo a ser bem entendido com o advento da teoria de campos em meados do século passado. Vamos considerar aqui o espalhamento de uma partícula leve, o elétron por exemplo, por um núcleo muito mais pesado, de carga $-Z e$, de forma que o recuo sofrido pelo núcleo pesado será considerado desprezível. Uma forma de representar esta interação é dada na Figura 1.4. O elétron se aproxima do núcleo

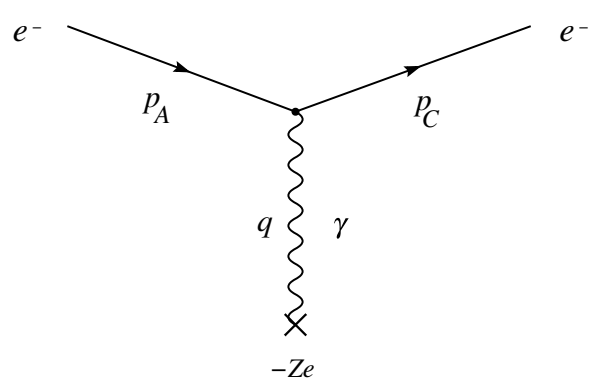

Figura 1.4: Espalhamento coulombiano entre um elétron e um núcleo pesado de carga $-Z e$.

com momento $p=p_{A}$, sofre a repulsão transmitida pelo fóton virtual $\gamma$, sendo desviado de seu caminho e seguindo com momento $p_{C}$. A seção de choque para este processo pode ser obtida de (1.11) considerando o núcleo $B$ em repouso antes e depois da colisão. Na aproximação $m_{B} c^{2}>E_{A}$ a seção de choque diferencial é dada por

$$
\frac{\mathrm{d} \sigma}{\mathrm{d} \Omega}=\left(\frac{\hbar}{8 \pi m_{B} c}\right)^{2}\left\langle|\mathcal{M}|^{2}\right\rangle
$$


onde \langle\rangle significa uma média sobre os spins das partículas. Para o espalhamento simples descrito pela Figura 1.4, a média do quadrado da amplitude será dada por $^{5}$

$$
\left\langle|\mathcal{M}|^{2}\right\rangle=\left(\frac{16 \pi e_{e}^{2} m_{B}}{\hbar q^{2}}\right)^{2}\left((m c)^{2}+|\vec{p}|^{2} \cos ^{2} \frac{\theta}{2}\right)
$$

onde $q^{2}=-4|\vec{p}|^{2} \sin ^{2}(\theta / 2)$. Se o elétron incidente for não-relativístico, ou seja, se $|\vec{p}|^{2}<<(m c)^{2}$, a seção de choque (1.23) se reduz à fórmula do espalhamento Rutherford

$$
\frac{\mathrm{d} \sigma}{\mathrm{d} \Omega}=\left(\frac{2 m e_{e}^{2}}{q^{2}}\right)^{2}=\frac{m^{2} e_{e}^{4}}{4|\vec{p}|^{4} \sin ^{4}(\theta / 2)},
$$

que como sabemos também pode ser obtida por considerações puramente clássicas. Mas como já discutimos anteriormente, este espalhamento também deve ter uma contribuição devido a polarização do vácuo. O diagrama que representa este processo é dado na Figura 1.5. Levando em conta esta contribuição, usando

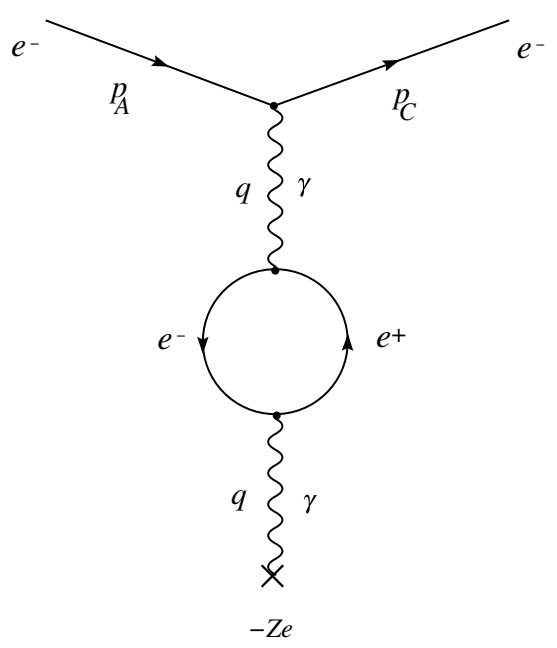

Figura 1.5: Contribuição de 1-loop para o espalhamento coulombiano.

os resultados (1.14)-(1.21), concluímos que a amplitude total do espalhamento fica modificada para

$$
\left\langle|\mathcal{M}|^{2}\right\rangle=\left(\frac{16 \pi m_{B}}{\hbar q^{2}} e_{R}^{2}\left\{1+\frac{e_{R}^{2}}{12 \pi^{2}} f\left(\frac{-q^{2}}{m^{2} c^{2}}\right)\right\}\right)^{2}\left((m c)^{2}+|\vec{p}|^{2} \cos ^{2} \frac{\theta}{2}\right) .
$$

Para o caso em que o espalhamento é de baixa energia, ou seja, o momento transferido $q$ é pequeno, usando o resultado $f(x) \approx x / 5$ dado em (C.14), vemos que a seção de choque de Rutherford (1.25) fica modificada para

$$
\frac{\mathrm{d} \sigma}{\mathrm{d} \Omega}=\left(\frac{2 m e_{R}^{2}}{q^{2}}\right)^{2}\left\{1-\frac{e_{R}^{2}}{60 \pi^{2}} \frac{q^{2}}{m^{2} c^{2}}\right\}^{2} .
$$

\footnotetext{
${ }^{5}$ Para mais detalhes desta dedução ver pág. 240 de [9]
} 
Para o caso estático, quando $q=0$, temos a simples repulsão de duas cargas coulombianas. Mas à medida que a transferência de momento $q$ aumenta, a seção de choque muda, indicando um desvio da lei de Coulomb. A carga elétrica $e_{R}$ muda de valor com o momento $q$, ou seja, com a escala de energia em que ocorre o processo físico. Podemos então escrever a carga como função do momento $q$, da seguinte forma:

$$
e_{R}\left(q^{2}\right)=e_{R}(0) \sqrt{1+\frac{e_{R}(0)^{2}}{12 \pi^{2}} f\left(\frac{-q^{2}}{m^{2} c^{2}}\right)}
$$

onde $e_{R}(0)$ representa a carga de repouso, ou seja, a carga que medimos no laboratório. Por isso, daqui para frente, usaremos $e_{R}(0) \equiv e$, pois esta é a carga real presente nos experimentos.

Esta variação da constante de acoplamento da interação eletromagnética é um fenômeno que acontece também na teoria das interações nucleares forte e fraca, e é daí que vem a idéia de que as forças fundamentais da natureza podem ser unificadas, pois as constantes de acoplamento de todas elas parecem convergir para um mesmo valor a altíssimas energias, conforme mostrado esquematicamente na Figura 1.6.

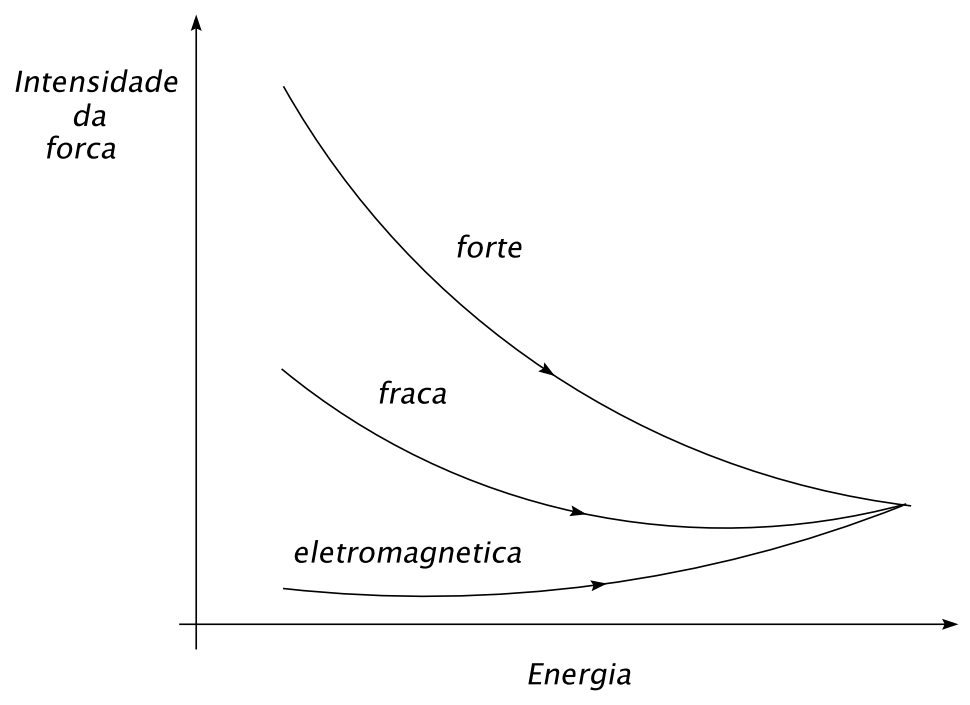

Figura 1.6: Variação das constantes de acoplamento das interações forte, fraca e eletromagnética.

Para terminar, note que não é difícil imaginar outros diagramas que contribuem também para o espalhamento coulombiano. Uma família de gráficos com 1-loop de férmion está indicada na Figura 1.7. Basta acrescentar cada vez mais fótons ligando o loop ao férmion espalhado. Embora as contribuições sejam cada vez menores, elas existem e devem ser consideradas a medida que resultados mais precisos são requeridos.

O desvio dado pela equação (1.26) é uma das contribuições para explicar corretamente o desvio nas linhas espectrais do átomo de hidrogênio, ou "Lamb shift", conforme citado na introdução. 

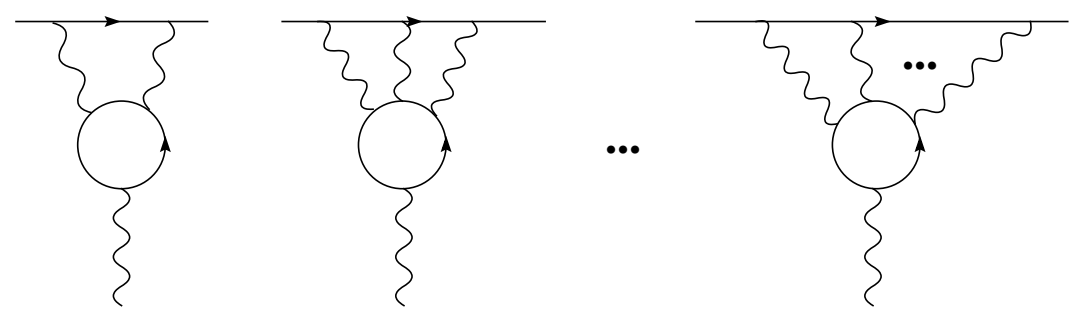

Figura 1.7: Outros diagramas de 1-loop fermiônico que contribuem para o espalhamento coulombiano.

\subsubsection{Funções de Green}

Vamos apresentar agora alguns aspectos mais formais da teoria de campos, mas que serão muito importantes para entendermos como é formulada a teoria de campos a temperatura finita. São as funções de Green, da qual as regras de Feynman originam-se naturalmente. Faremos uma exposição breve, apresentando apenas os resultados necessários para a inclusão de efeitos de temperatura ${ }^{6}$.

Em mecânica quântica, uma quantidade de extrema importância no estudo de um sistema é a probabilidade de transição, ou amplitude de probabilidade, de que o sistema faça uma transição de um estado inicial $\left|q^{i}, t^{i}\right\rangle$ para um estado final $\left|q^{f}, t^{f}\right\rangle$, e é dada por

$$
\left\langle q^{f}, t^{f} \mid q^{i}, t^{i}\right\rangle=\left\langle q^{f}\left|\mathrm{e}^{-\frac{i}{\hbar} \hat{H}\left(t^{f}-t^{i}\right)}\right| q^{i}\right\rangle,
$$

onde $q$ representa a coordenada generalizada que descreve o sistema e $\hat{H} \equiv \hat{H}(\hat{q}, \hat{p})$ é a hamiltoniana escrita em termos dos operadores $\hat{q}$ e $\hat{p}$ (momento canonicamente conjugado a $\hat{q}$ ). Esta amplitude de transição pode ser calculada usando-se o método das integrais de trajetória desenvolvido por Feynman, e pode-se mostrar que $[2,10]$

$$
\left\langle q^{f}, t^{f} \mid q^{i}, t^{i}\right\rangle \propto \int D q \int D p \exp \left[\frac{i}{\hbar} \int_{t^{i}}^{t^{f}} d t(p \dot{q}-H(p, q))\right]
$$

onde $H(q, p)$ não é mais um operador e está escrito em termos dos autovalores $q$ e $p$. A integração nos diz que devemos "somar" sobre todos os caminhos possíveis $q(t)$ que satisfaçam às condições de contorno

$$
q\left(t^{i}\right)=q^{i}, \quad q\left(t^{f}\right)=q^{f}
$$

e sobre todos os momentos possíveis da partícula entre estes pontos, o que significa que nos instantes inicial $t^{i}$ e final $t^{f}$ a partícula está em uma posição bem determinada $q^{i}$ e $q^{f}$, respectivamente. O sinal de proporcionalidade está sendo usado para indicar que existe ainda um fator de normalização, mas ele não é importante para nossa discussão.

Em teoria de campos, sabemos que a coordenada generalizada $q(t)$ deve ser substituída (generalizando

\footnotetext{
${ }^{6}$ Para uma exposição completa indicamos $[1,2,3,10]$, embora a maioria dos livros de teoria de campos tratem deste assunto.
} 
para o espaço tridimensional) por um campo $\varphi(t, \vec{x})$. Desta forma, a generalização de (1.30) é

$$
\left\langle\varphi^{f}(\vec{x}), t^{f} \mid \varphi^{i}(\vec{x}), t^{i}\right\rangle \propto \int D \varphi \int D \pi \exp \left[\frac{i}{\hbar} \int_{t^{i}}^{t^{f}} \mathrm{~d} t \int \mathrm{d}^{3} x\left(\pi \partial_{0} \varphi-\mathscr{H}(\pi, \varphi)\right)\right],
$$

onde $\mathscr{H}$ é a densidade de hamiltoniana, $\pi=\partial \mathscr{L} / \partial\left(\partial_{0} \varphi\right)$ é o momento conjugado de $\varphi, \mathscr{L}$ é a densidade de lagrangeana e $\partial_{0} \equiv \partial / \partial x_{0}$. A integral de trajetória é sobre todas as funções $\pi(t, \vec{x})$ (autovalores de $\hat{\pi}$ ), e sobre as funções $\varphi(t, \vec{x})$ (autovalores de $\hat{\varphi}$ ) que satisfaçam

$$
\varphi\left(t^{f}, \vec{x}\right)=\varphi^{f}(\vec{x}) \quad \varphi\left(t^{i}, \vec{x}\right)=\varphi^{i}(\vec{x}) .
$$

Estas são as condições de contorno análogas de (1.31). Em geral, ou pelo menos na maioria dos casos em que estamos interessados, a densidade de hamiltoniana é apenas quadrática no momento $\pi$, de forma que a integral funcional nesta variável pode ser feita, e ficamos com

$$
\left\langle\varphi^{f}(\vec{x}), t^{f} \mid \varphi^{i}(\vec{x}), t^{i}\right\rangle=N \int D \varphi \exp \left[\frac{i}{\hbar} \int_{t^{i}}^{t^{f}} \mathrm{~d} t \int \mathrm{d}^{3} x \mathscr{L}\left(\varphi, \partial_{\mu} \varphi\right)\right],
$$

onde agora tanto o fator de proporcionalidade anterior quanto o resultado da integração em $\pi$ estão representados por $N$. Note que o argumento da exponencial é algo conhecido, é proporcional à ação $S$ do sistema ${ }^{7}$.

Mas esta não é uma quantidade muito útil para cálculos explícitos, pois em geral não sabemos em que estado $|\varphi(\vec{x}), t\rangle$ se encontra o sistema. O que conhecemos de um sistema quântico são seus autovalores de energia e os respectivos auto-estados. Um estado importante é o estado de menor energia, ou estado fundamental, ou ainda estado de vácuo $|0\rangle$. Uma quantidade que nos interessa é amplitude de probabilidade de que o sistema faça uma transição, sob ação de alguma interação externa $J(t, \vec{x})$, do estado fundamental, em $t^{i}$, para o próprio estado fundamental, em $t^{f}$. Isto é conhecido como amplitude de transição vácuovácuo sobre influência da interação $J$, e é representado por $Z[J] \equiv\langle 0 \mid 0\rangle^{J}$. Isto pode ser calculado partindo-se de (1.34). Não vamos mostrar os detalhes do cálculo ${ }^{8}$, o que nos interessa é somente a expressão final, e é dada por

$$
Z[J] \equiv\langle 0 \mid 0\rangle^{J}=N \lim _{\substack{t^{i} \rightarrow-T \mathrm{e}^{-i \delta} \\ t f \rightarrow T \mathrm{e}^{-i \delta}}} \int D \varphi \exp \left[\frac{i}{\hbar} \int_{t^{i}}^{t^{f}} \mathrm{~d} t \int \mathrm{d}^{3} x(\mathscr{L}+J \varphi)\right]
$$

Neste ponto, alguns comentários se fazem necessários. A expressão (1.35) só faz sentido se a integração no tempo for feita através de uma rotação para eixo imaginário, caracterizado pelo parâmetro $\delta$. Isto acontece por causa de problemas de convergência ${ }^{9}$ que aparecem nos cálculos intermediários entre (1.34) e (1.35). Se $\delta=\pi / 2$ e $T \rightarrow \infty$, de maneira que o tempo varia em todo o eixo imaginário, isto é conhecido

\footnotetext{
${ }^{7}$ Classicamente temos $S=\int d t L(q, \dot{q})$

${ }^{8}$ Para uma boa discussão, vide pags. 174-177 de [2] por exemplo.

${ }^{9}$ Basicamente o que acontece é que, como o argumento da exponencial é um número complexo, a exponencial oscila quando $t$ varia, de forma que não se pode assegurar sua convergência. Já no eixo imaginário a exponencial se torna real e decrescente, de forma que a convergência fica garantida.
} 
como rotação de Wick, que corresponde a uma rotação do espaço de Minkowski para o espaço Euclidiano, conforme Figura 1.8. Mas isto também não é um problema, trata-se apenas de um artifício matemático para garantir a convergência da integral, e depois de todos os cálculos terem sido feitos devemos voltar para o eixo real do tempo. Quanto ao fator de normalização $N$, ele é escolhido de forma que $Z[J]=1$ quando $J=0$.

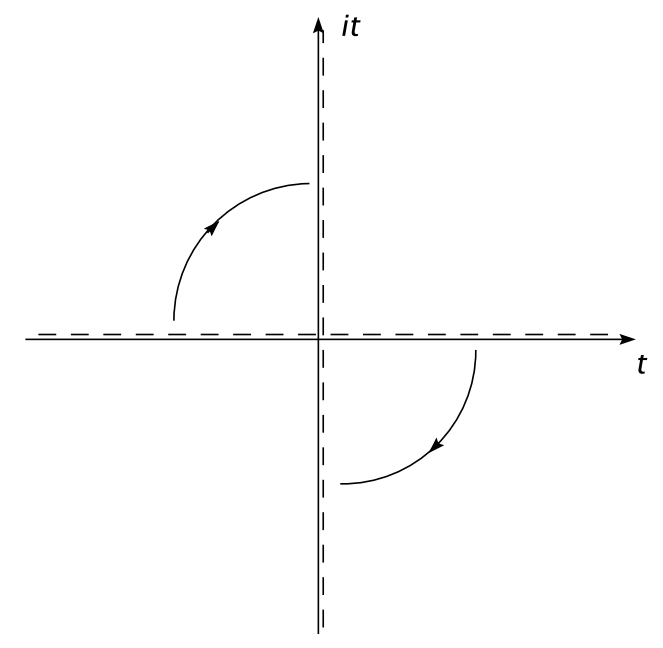

Figura 1.8: Rotação de Wick para o eixo imaginário do tempo.

Com isto, podemos introduzir uma quantidade de extrema importância em teoria de campos, conhecida como função de Green de n-pontos, denotada por

$$
\mathcal{G}^{(n)}\left(x_{1}, \cdots, x_{n}\right) \equiv\left\langle 0\left|T\left[\hat{\varphi}\left(x_{1}\right) \cdots \hat{\varphi}\left(x_{n}\right)\right]\right| 0\right\rangle,
$$

que nada mais é que o valor esperado no vácuo do produto ordenado no tempo de $n$ operadores de campo $\hat{\varphi}$. Através de derivadas funcionais de $Z[J]$ não é difícil mostrar que

$$
\left\langle 0\left|T\left[\hat{\varphi}\left(x_{1}\right) \cdots \hat{\varphi}\left(x_{n}\right)\right]\right| 0\right\rangle=\left.\left(\frac{\hbar}{i}\right)^{n} \frac{\delta^{n} Z[J]}{\delta J\left(x_{1}\right) \cdots \delta J\left(x_{n}\right)}\right|_{J(x)=0}
$$

onde $x_{i} \equiv\left(x_{i}^{0}, \vec{x}_{i}\right), \quad i=1, \cdots, n$ e $T[\cdots]$ representa o ordenamento temporal dos campos $\hat{\varphi}$.

Este é o resultado final a que queríamos chegar. Dada uma densidade de lagrangeana $\mathscr{L}$, através da equação (1.35) podemos calcular a amplitude de transição vácuo-vácuo, e com ela, pelas (1.36) e (1.37) podemos calcular as funções de Green de $n$-pontos. Mas na verdade as coisas não são tão fáceis assim. O cálculo exato de $Z[J]$ só é possível para o caso de teorias livres, sem interações, e o que nos interessa é exatamente o caso em que as interações estão presentes. Por exemplo, queremos saber como se dá a interação do fóton (campo eletromagnético) com os elétrons (partículas fermiônicas). A solução a este problema também é conhecida, o que se faz é separar a lagrangeana em uma parte livre, $\mathscr{L}_{0}$, e em uma parte de interação $\mathscr{L}_{I}$. A parte de interação deve ser expandida em potências da constante de acoplamento 
da interação (carga elétrica no caso da eletrodinâmica), e o problema é então tratado perturbativamente. Por exemplo, gostaríamos de saber quais são exatamente os propagadores do fóton e do elétron para uma teoria com interação (lembre-se que as expressões dadas em (1.2) e (1.3) são os propagadores livres). Na falta de uma resposta exata, o tratamento perturbativo nos diz que o propagador exato do fóton por exemplo pode ser expresso diagramaticamente ${ }^{10}$ pela Figura 1.9. O primeiro termo desta expansão é o propagador livre

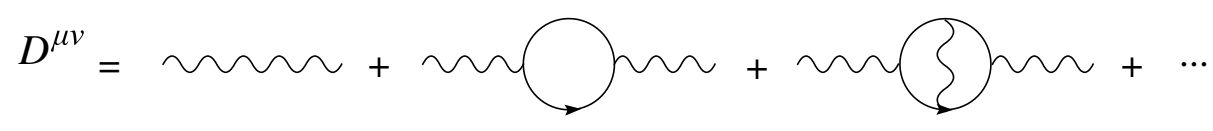

Figura 1.9: Expansão perturbativa para o propagador do fóton. O primeiro diagrama corresponde ao propagador livre $D_{0}^{\mu \nu}$.

(ordem zero em $e$ ), equação (1.2), o segundo termo é a auto-energia do fóton (ordem $e^{2}$ ), o terceiro termo é um diagrama de 2-loops (ordem $e^{4}$ ), e um número infinito de diagramas contribuem para esta expansão. Portanto, o interesse em se conhecer as funções de Green é saber exatamente quais os diagramas que contribuem para um processo, dada uma interação. Como já foi citado nas seções anteriores, o diagrama de auto-energia do fóton é a primeira correção perturbativa a um processo direto, e é neste tipo de diagrama que estaremos interessados nos capítulos seguintes.

Outros dois diagramas de 1-loop muito importantes nos estudos da eletrodinâmica quântica são os diagramas de auto-energia do fóton e o de correção de vértice, mostrados nas Figuras 1.10 e 1.11. O primeiro dará uma contribuição para a renormalização da massa do elétron, enquanto o segundo é a principal contribuição para explicar o momento magnético anômalo do elétron.

Como vimos brevemente nesta seção, os diagramas de Feynman são uma ferramenta importante para se obter resultados precisos em física de altas energias, principalmente quando os processos envolvidos são processos de espalhamento e produção de partículas. A ligação entre a teoria e o experimento é feita por meio das seções de choque que descrevem o fenômeno, e o cálculo preciso destas seções de choque envolve o cálculo de diagramas de 1-loop por exemplo, que são as primeiras correções perturbativas a um dado processo. À medida que as técnicas experimentais avançam, torna-se cada vez mais possível testar uma teoria olhando para suas contribuições ou modificações nas seções de choque correspondentes. Teorias que envolvem interações entre partículas são as mais interessantes, e como vimos, termos de interação darão origem a vértices nas regras de Feynman, que conseqüentemente darão contribuições a diagramas de 1loop, por isso o interesse no estudo de tais digramas. Quando tratarmos da QED não-comutativa no próximo capítulo, ficará claro que uma possível maneira de se testar a existência ou não da não-comutatividade das coordenadas é por meio do cálculo de seções de choque de determinados processos por exemplo. A não-comutatividade dará origem a novos termos de interação, que por sua vez contribuirão com novos diagramas que podem ser significativos no cálculo de determinadas seções de choque.

Agora vamos discutir um pouco a teoria de campos a temperatura finita.

\footnotetext{
${ }^{10}$ A expressão matemática deste diagrama segue diretamente da função de Green de 2-pontos, os detalhes estão nos livros de teoria de campos, queremos apenas mostrar qualitativamente o que acontece.
} 


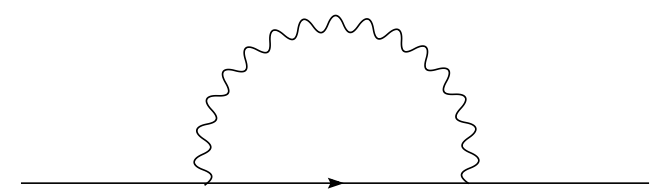

Figura 1.10: Auto-energia do elétron.

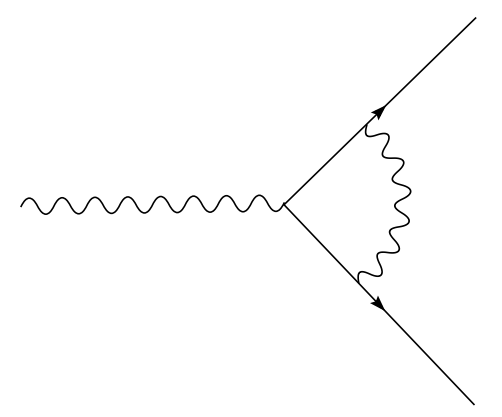

Figura 1.11: Correção de vértice.

\subsection{QED a temperatura finita}

Antes de iniciarmos nossos estudos da teoria quântica de campos a temperatura finita, vamos apresentar algumas questões que se relacionam a ela.

O que acontece com a matéria ordinária quando ela é submetida a altíssima pressão de tal maneira que os elétrons que a compõe formam um gás degenerado relativístico? É isto o que acontece em estrelas anãs brancas por exemplo. E quando os núcleos são comprimidos a ponto de se juntarem para formar uma matéria nuclear superdensa, como nas estrelas de nêutrons? E quando a matéria nuclear é aquecida a altíssimas temperaturas de forma que os prótons e nêutrons se quebram em quarks e glúons? É isso que se supõe acontecer em uma colisão núcleo-núcleo de alta energia. O que acontece com a quebra espontânea de simetria da teoria unificada eletrofraca durante o big bang? Questões como estas fascinaram os físicos nas últimas décadas, e as respostas envolvem estudos de mecânica estatística, de física de partículas elementares, física nuclear, astrofísica e cosmologia. Sistemas como esses estão fora do alcance das experiências humanas mas o universo está aí como um grande laboratório. Portanto, para entendermos um pouco sobre os mecanismos que agem sob condições tão extremas de temperatura e densidade, necessitamos de uma formulação da teoria de campos que leve em conta tais aspectos. As ferramentas estão na teoria de campos a temperatura finita.

Teorias de campos não relativísticas a temperatura e densidade finitas apareceram por volta de 1950 para descrever sistemas da matéria condensada em condições de laboratório, ou seja, fora do regime extremo de altíssimas densidades e temperaturas. Os conceitos envolvidos eram baseados apenas na equação de Schrödinger e em mecânica estatística, já que se tratava de sistemas de muitas partículas. Isto ficou conhecido como "Problema de muitos corpos". A teoria de campos relativística a temperatura finita foi 
primeiramente estudada por Fradkin em 1965 e redescoberta dez anos depois, motivada pela busca de uma descrição da transição de fase que deve ocorrer com a teoria eletrofraca a uma energia da ordem de 200 $\mathrm{MeV}$, de grande interesse para se entender a história do universo, pois esta transição de fase pode ter desempenhado um papel importante na formação de matéria escura, que é um dos grandes problemas da cosmologia moderna. Por volta de 1980, estudos de teoria de campos na rede sugeriram a existência de uma fase da matéria nuclear em que quarks e glúons poderiam existir separadamente, o chamado plasma de quarks e glúons, a uma energia estimada da ordem de $150 \mathrm{MeV}$, conforme ilustrado na introdução pelo diagrama de fases. A possibilidade da observação deste novo estado da matéria em colisões ultra-relativísticas de íons pesados deu um novo impulso ao estudo da teoria de campos a temperatura finita.

Nosso objetivo aqui é dar uma breve introdução a este assunto do ponto de vista da eletrodinâmica quântica.

\subsubsection{Revisão de termodinâmica}

O principal objeto do estudo da termodinâmica de sistemas em equilíbrio ou da mecânica estatística é a função de partição $Z$. Dela derivam todas as quantidades macroscópicas de interesse do sistema. A função de partição que caracteriza o ensemble "grande canônico" é dada por

$$
\begin{aligned}
Z & =\operatorname{Tr} \exp [-\beta(\hat{H}-\mu \hat{N})] \\
& =\sum_{n}\left\langle n\left|\mathrm{e}^{-\beta(\hat{H}-\mu \hat{N})}\right| n\right\rangle,
\end{aligned}
$$

com

$$
\hat{H}|n\rangle=E_{n}|n\rangle
$$

e

$$
\hat{N}|n\rangle=n|n\rangle
$$

onde $|n\rangle$ e $E_{n}$ são, respectivamente, os auto-estados e autovalores da energia do hamiltoniano $\hat{H}$, e $\hat{N}$ representa o operador "número de partículas" do sistema, caracterizando o número $n$ de partículas que compõe determinado estado. O potencial químico $\mu$ caracteriza a possibilidade do sistema trocar partículas com o meio externo. O caso $\mu=0$ representa o ensemble canônico, caracterizado por um número fixo de partículas. Vamos trabalhar no sistema de unidades onde a constante de Boltzmann é igual a unidade, de forma que $\beta=1 / k_{B} T \equiv 1 / T$ representa o inverso da temperatura. De posse da função de partição, podemos determinar quantidades como a pressão $P$, a entropia $S$, o número médio de partículas $N$ e a 
energia interna $E$ pelas relações

$$
\begin{array}{ll}
P=T \frac{\partial \ln Z}{\partial V}, & N=T \frac{\partial \ln Z}{\partial \mu}, \\
S=\frac{\partial(T \ln Z)}{\partial T}, & E=-P V+T S+\mu N .
\end{array}
$$

Uma outra quantidade muito importante é a energia livre de Helmholtz $F$, que se relaciona à energia interna por meio de

$$
F=E-T S,
$$

e também deriva da função de partição

$$
F=-\frac{1}{\beta} \ln Z
$$

Se tivermos um sistema unidimensional composto por bósons livres, por exemplo um gás de bósons, teremos $E_{n}=\hbar \omega\left(n+\frac{1}{2}\right)$, onde $n=0,1,2, \cdots \infty$. Por simplicidade vamos adotar $\hbar=1$ e desconsiderar o fator $1 / 2$, que representa apenas a energia de ponto zero do sistema, de maneira que $E_{n}=n \omega$. A função de partição (1.38) fica

$$
\begin{aligned}
Z & =\sum_{n=0}^{\infty} \mathrm{e}^{-\beta(\omega-\mu) n} \\
& =\frac{1}{1-\mathrm{e}^{-\beta(\omega-\mu)}},
\end{aligned}
$$

e o número médio de bósons será

$$
N=T \frac{\partial \ln Z}{\partial \mu}=\frac{1}{\mathrm{e}^{\beta(\omega-\mu)}-1}
$$

No limite $\mu=0$ temos a função distribuição de Bose-Einstein $n_{B}$,

$$
n_{B}(\omega)=\frac{1}{\mathrm{e}^{\beta \omega}-1} .
$$

Para o caso de um gás de férmions, temos $n=0,1$, de maneira que a função de partição fica

$$
\begin{aligned}
Z & =\sum_{n=0}^{1} \mathrm{e}^{-\beta(\omega-\mu) n} \\
& =1+\mathrm{e}^{-\beta(\omega-\mu)}
\end{aligned}
$$

e o número médio de férmions

$$
N=T \frac{\partial \ln Z}{\partial \mu}=\frac{1}{\mathrm{e}^{\beta(\omega-\mu)}+1} .
$$


No caso $\mu=0$ temos a função distribuição de Fermi-Dirac $n_{F}$,

$$
n_{F}(\omega)=\frac{1}{\mathrm{e}^{\beta \omega}+1} .
$$

\subsubsection{Funções de Green a temperatura finita}

Vamos ver agora como ficam as regras de Feynman apresentadas na primeira seção devido à presença da temperatura. Já vimos no caso a temperatura nula que as regras de Feynman são derivadas através do estudo das funções de Green de $n$-pontos do sistema, então nosso objetivo é apresentar qual o análogo das funções de Green para o caso a temperatura finita. No caso anterior, somente o estado de vácuo tinha um papel importante, só que agora todos os estados devem contribuir para o funcional gerador das funções de Green, cada um com um peso estatístico diferente, dependente de sua energia. Desta forma, a generalização correta de (1.36) deve ser

$$
\mathcal{G}^{(n)}\left(x_{1}, \cdots, x_{n}\right) \equiv \sum_{j} \mathrm{e}^{-\beta E\left(\varphi_{j}\right)}\left\langle\varphi_{j}\left|T\left[\hat{\varphi}\left(x_{1}\right) \cdots \hat{\varphi}\left(x_{n}\right)\right]\right| \varphi_{j}\right\rangle
$$

onde a soma é sobre um conjunto completo de estados $\left|\varphi_{j}\right\rangle$ com energia $E\left(\varphi_{j}\right)$. Isto nada mais é que o valor médio do produto ordenado no tempo de $n$-operadores de campo no ensemble grande canônico com potencial químico igual a zero

$$
\mathcal{G}^{(n)}\left(x_{1}, \cdots, x_{n}\right)=\frac{\operatorname{Tr} \mathrm{e}^{-\beta \hat{H}} T\left[\hat{\varphi}\left(x_{1}\right) \cdots \hat{\varphi}\left(x_{n}\right)\right]}{\operatorname{Tr} \mathrm{e}^{-\beta \hat{H}}}
$$

onde $\hat{H}$ é a hamiltoniana do sistema. Para entendermos quais as mudanças que ocorrem nas regras de Feynman, é mais fácil usarmos de um artifício matemático que ficou conhecido por formalismo do tempo imaginário. Note que se fizermos $t_{f}-t_{i}=-i \beta$ na equação (1.29), aquela amplitude fica tendo a mesma cara da função de partição se os estados $|q\rangle$ forem auto-estados da energia. O fato do tempo variar no eixo complexo significa que devemos ir para o espaço euclidiano. Desta forma, tudo o que foi feito para temperatura zero é válido desde que o intervalo temporal, antes variando de $[-\infty, \infty]$, agora fique restrito ao intervalo $[0, \beta]$. Isto faz com que a expansão de Fourier de um campo no eixo temporal seja restrita a um intervalo finito, portanto ao invés de usarmos uma integração devemos usar um somatório para escrevermos a parte temporal da expansão de Fourier de um campo. Desta forma, a regra de Feynman número 6 da seção 1.1.1 fica modificada para:

9 - Soma sobre freqüências - No espaço euclidiano, em um loop interno, as freqüências devem ser somadas

$$
\int \frac{\mathrm{d}^{4} k}{(2 \pi)^{4}} \rightarrow \frac{1}{\beta} \sum_{n} \int \frac{\mathrm{d}^{3} k}{(2 \pi)^{3}}
$$


onde $k_{0} \equiv i \omega_{n}$ no espaço euclidiano e

$$
\omega_{n}=\left\{\begin{array}{cl}
\frac{2 n \pi}{\beta}, & \text { bósons } \\
\frac{(2 n+1) \pi}{\beta}, & \text { férmions }
\end{array}\right.
$$

Estas são as chamadas freqüências de Matsubara. Vários livros texto discutem de forma bastante clara a formulação da teoria de campos a temperatura finita, por isso não vamos nos deter nestes detalhes técnicos. Vamos apenas aplicar os métodos já desenvolvidos e muito bem estabelecidos desta teoria a problemas de nosso interesse. Para o leitor interessado indicamos os livros de J. I. Kapusta [11], M. Le Bellac [12] e A. Das [13].

\subsubsection{Função de partição}

Desta breve introdução à teoria de campos a temperatura finita, fica claro a estreita analogia com o caso a temperatura zero. As funções de Green podem ser definidas de maneira semelhante, os diagramas que contribuem para um processo são semelhantes aos encontrados no caso anterior, o que difere é o método de cálculo dos diagramas, como ficará claro quando fizermos o cálculo do tensor de auto-energia do fóton. Mas existe uma outra quantidade de extrema importância que podemos calcular, agora usando a teoria de campos a temperatura finita, que é função de partição de um sistema. Conforme descrito na seção 1.2.1, de posse da função de partição podemos derivar relações importantes da termodinâmica, como pressão, entropia, energia interna, etc.

Vamos fazer uma aplicação ao caso de um gás de elétrons e um gás de fótons a altas temperaturas, usando as ferramentas da teoria de campos a temperatura finita.

\section{Gás de elétrons}

Vamos considerar novamente a função de partição de um sistema quântico, equação (1.38), no caso de potencial químico nulo:

$$
Z=\sum_{n}\left\langle n\left|\mathrm{e}^{-\beta \hat{H}}\right| n\right\rangle
$$

Note a semelhança desta expressão com a (1.29), que foi o ponto de partida para a obtenção da função de Green. Naquele caso, o intervalo de tempo $t_{f}-t_{i}$ não desempenhou nenhum papel importante, pois de acordo com (1.35), estes intervalos foram tomados tendendo ao infinito de forma que a única contribuição para a amplitude veio dos estados de vácuo. Aqui o tempo também não desempenha nenhum papel importante, pois estamos tratando de sistemas em equilíbrio. A pergunta agora é: Qual o análogo da função de partição (1.53) para o caso de férmions livres do ponto de vista da teoria de campos? A resposta é:

$$
Z=\sum_{\psi(\vec{x})}\left\langle\psi(\vec{x})\left|\mathrm{e}^{-\beta \hat{H}}\right|-\psi(\vec{x})\right\rangle
$$


Mas porque o sinal negativo no campo do lado direito? Podemos afirmar que isso é específico para o caso de férmions. Os observáveis físicos sempre envolvem potências pares dos campos de Dirac $\psi$, já que estes mudam de sinal por uma rotação de $2 \pi$. Assim, os auto-estados $| \pm \psi(\vec{x})\rangle$ do operador $\hat{\psi}(\vec{x})$ correspondem ao mesmo valor dos observáveis físicos e descrevem o mesmo estado. Para evitar problemas e sermos consistente com a estatística de Fermi, devemos tomar como ponto de partida a expressão (1.54). O desenvolvimento agora pode ser feito analogamente ao caso da seção 1.1.5, levando em conta que agora estamos trabalhando no eixo imaginário do tempo, com a seguinte mudança de variáveis:

$$
\tau=i t=i x^{0}
$$

com $t^{i}=0$ e $t^{f}=-i \beta$. Isso corresponde a definirmos $\bar{x}^{\mu} \equiv(\tau, \overline{\vec{x}})=\left(i x^{0}, \vec{x}\right)=(-i \tau, \vec{x})($ estamos usando $c=1$ ). Podemos mostrar então que

$$
Z=N(\beta) \int_{\text {antiperiod }} D \bar{\psi} D \psi \exp \int_{0}^{\beta} \mathrm{d} \tau \int \mathrm{d}^{3} x \mathcal{L}(\bar{\psi}, \psi)
$$

onde em $\mathcal{L}$ o campo $\psi$ é entendido como sendo função de $\tau$ e $\vec{x}$, e é anti-periódico no intervalo $0<\tau<\beta$,

$$
\psi(\tau=0, \vec{x})=-\psi(\tau=\beta, \vec{x}) .
$$

A expansão de Fourier apropriada para $\psi(\bar{x})$ é

$$
\psi(\bar{x})=\frac{1}{\beta} \sum_{n} \int \frac{\mathrm{d}^{3} p}{(2 \pi)^{d}} \mathrm{e}^{-i \bar{p} \cdot \bar{x}} \tilde{\psi}(\bar{p}),
$$

onde $\bar{p}^{\mu} \equiv\left(\bar{p}^{0}, \overline{\vec{p}}\right)=\left(-i p^{0}, \vec{p}\right)$ e as frequiências de Matsubara para férmions são

$$
\omega_{n}=\frac{(2 n+1) \pi}{\beta}
$$

$\operatorname{com} p^{0} \equiv i \omega_{n}$ e $n$ inteiro.

Para o caso de um campo livre, a lagrangeana apropriada é a lagrangeana de Dirac, escrita em termos da variável $\bar{x}$,

$$
\mathcal{L}(\bar{\psi}, \psi)=\bar{\psi}(\bar{x})\left(i \gamma^{\mu} \bar{\partial}_{\mu}-m\right) \psi(\bar{x})
$$

de forma que temos

$$
Z=N(\beta) \int_{\text {antiperiod }} D \bar{\psi} D \psi \exp \left(-\int \mathrm{d}^{4} \bar{x}^{\prime} \int \mathrm{d}^{4} \bar{x} \bar{\psi}\left(\bar{x}^{\prime}\right) \mathrm{D}\left(\bar{x}^{\prime}, \bar{x}\right) \psi(\bar{x})\right)
$$

com

$$
\mathrm{D}\left(\bar{x}^{\prime}, \bar{x}\right)=\left(i \gamma^{\mu} \bar{\partial}_{\mu}+m\right) \delta^{4}\left(\bar{x}^{\prime}-\bar{x}\right)
$$


onde $\bar{\partial}_{\mu} \psi \equiv(i \partial \psi / \partial \tau, \nabla \psi)$. Usando a propriedade ${ }^{11}$

$$
\int D \phi \exp \left(-\frac{1}{2} \int \mathrm{d} x^{\prime} \int \mathrm{d} x \phi\left(x^{\prime}\right) A\left(x^{\prime}, x\right) \phi(x)\right)=\exp \left(-\frac{1}{2} \operatorname{Tr} \ln A\right),
$$

onde $A$ é a matriz que representa $A\left(x^{\prime}, x\right)$, podemos então escrever

$$
Z=N(\beta) \exp (\operatorname{Tr} \ln \mathrm{D})
$$

onde o traço $\operatorname{Tr}$ significa $\int \mathrm{d} \tau \int \mathrm{d}^{3} x$ tr e tr significa soma sobre os índices de Dirac. Escrevendo

$$
\delta^{4}\left(\bar{x}^{\prime}-\bar{x}\right) \equiv \delta\left(\tau^{\prime}-\tau\right) \delta^{3}\left(\vec{x}^{\prime}-\vec{x}\right)
$$

e representando $\delta^{4}\left(\bar{x}^{\prime}-\bar{x}\right)$ por uma transformada de Fourier da forma

$$
\delta^{4}\left(\bar{x}^{\prime}-\bar{x}\right)=\frac{1}{\beta} \sum_{n} \int \frac{\mathrm{d}^{3} p}{(2 \pi)^{3}} \mathrm{e}^{-i \bar{p} \cdot\left(\bar{x}^{\prime}-\bar{x}\right)},
$$

obtemos

$$
\mathrm{D}\left(\bar{x}^{\prime}, \bar{x}\right)=\frac{1}{\beta} \sum_{n} \int \frac{\mathrm{d}^{3} p}{(2 \pi)^{3}} \mathrm{e}^{-i \bar{p} \cdot\left(\bar{x}^{\prime}-\bar{x}\right)}(-\bar{p}+m) .
$$

Com isto

$$
\begin{aligned}
\operatorname{Tr} \ln \mathrm{D} & =\int_{0}^{\beta} \mathrm{d} \tau \int \mathrm{d}^{3} x \frac{1}{\beta} \sum_{n} \int \frac{\mathrm{d}^{3} p}{(2 \pi)^{3}} 2 \ln \left(m^{2}-\bar{p}^{2}\right) \\
& =2 \int \mathrm{d}^{3} x \sum_{n} \int \frac{\mathrm{d}^{3} p}{(2 \pi)^{3}} \ln \left(\omega_{n}^{2}+\vec{p}^{2}+m^{2}\right),
\end{aligned}
$$

onde usamos a identidade $\operatorname{tr} \ln (-\bar{p}+m)=2 \ln \left(m^{2}-\bar{p}^{2}\right)$.

A soma sobre as freqüências pode ser feita usando-se a identidade

$$
\frac{\mathrm{d}}{\mathrm{d} x}\left(\sum_{n=-\infty}^{\infty} \ln \left(\omega_{n}^{2}+x^{2}\right)\right)=2 x \sum_{n=-\infty}^{\infty} \frac{1}{\omega_{n}^{2}+x^{2}}
$$

e os resultados do Apêndice B.3. Fazendo isso chegamos a

$$
\operatorname{Tr} \ln \mathrm{D}=2 \int \mathrm{d}^{3} x \int \frac{\mathrm{d}^{3} p}{(2 \pi)^{3}}\left\{\beta \sqrt{\vec{p}^{2}+m^{2}}+2 \ln \left[1+\exp \left(-\beta \sqrt{\vec{p}^{2}+m^{2}}\right)\right]+C_{1}(\beta)\right\}
$$

onde $C_{1}(\beta)$ é uma constante dependente da temperatura mas independente de $\vec{p}$. Na verdade esta é uma constante infinita, pois se ela não depende de $\vec{p}$ a integral em $\mathrm{d}^{3} p$ diverge. No entanto pode-se mostrar que

\footnotetext{
${ }^{11}$ Vide págs. 1-4 de [3] por exemplo.
} 
este fator cancela exatamente o termo $N(\beta)$ ainda presente na função de partição. Este cancelamento é um fato notável ${ }^{12}$, mostrando a consistência da formulação por meio de integrais de trajetória. A integral em $\mathrm{d}^{3} x$ representa o volume $V$ do sistema. A função de partição, portanto, fica dada por:

$$
Z=\exp \left(4 \beta V \int \frac{\mathrm{d}^{3} p}{(2 \pi)^{3}}\left(\frac{1}{2} \sqrt{\vec{p}^{2}+m^{2}}+\frac{1}{\beta} \ln \left[1+\exp \left(-\beta \sqrt{\vec{p}^{2}+m^{2}}\right)\right]\right)\right)
$$

e a energia livre de Helmholtz (1.43)

$$
F=-4 V \int \frac{\mathrm{d}^{3} p}{(2 \pi)^{3}}\left(\frac{1}{2} \sqrt{\vec{p}^{2}+m^{2}}+\frac{1}{\beta} \ln \left[1+\exp \left(-\beta \sqrt{\vec{p}^{2}+m^{2}}\right)\right]\right)
$$

Quando a massa do campo fermiônico é desprezível comparada com a temperatura, podemos calcular a integral explicitamente. O primeiro termo na integral dá uma contribuição infinita, que representa uma energia de ponto zero, portanto deve ser cancelada por algum procedimento de renormalização. Tomando o limite $T>>m$, que equivale a $\beta m<<1$, obtemos

$$
Z=\exp \left(V \frac{7 \pi^{2} T^{3}}{180}\right), \quad F=-V \frac{7 \pi^{2} T^{4}}{180}
$$

de maneira que a pressão, a densidade de entropia e a densidade de energia são, usando (1.41),

$$
\begin{aligned}
P & =\frac{7 \pi^{2} T^{4}}{180} \\
\frac{S}{V} & =\frac{7 \pi^{2} T^{3}}{45} \\
\frac{E}{V} & =\frac{7 \pi^{2} T^{4}}{60}
\end{aligned}
$$

como esperado, ou seja, com dependência do tipo $T^{4}$ para a energia.

\section{Gás de fótons}

Para o caso de um gás de fótons o tratamento é semelhante, só que devemos tomar alguns cuidados. Intuitivamente, poderíamos tomar a expressão para a função de partição análoga a de um campo escalar, só levando em conta que temos um campo vetorial com quatro componentes, $A^{\mu}=\left(A^{0}, A^{1}, A^{2}, A^{3}\right)$. Mas existe um problema. O campo vetorial sem massa (fóton) possui apenas 2 graus de liberdade independentes ( 2 transversais), embora uma lagrangeana típica que seja renormalizável possua 4 graus de liberdade (2 transversais, 1 longitudinal, 1 tipo tempo). Os dois graus de liberdade extras não são físicos, e diz-se que eles não podem estar em equilíbrio térmico com o meio. Em vista destas particularidades, percebemos que $\operatorname{Tr} \mathrm{e}^{-\beta \hat{H}}$ não é uma quantidade de significado físico em todos os gauges. Em alguns gauges aparecem

\footnotetext{
${ }^{12}$ Uma boa discussão sobre isso é feita por Bernard em [14].
} 
partículas que representam graus de liberdade extras, que não podem estar em equilíbrio térmico e não devem ser levados em consideração. Se fizermos os cálculos num gauge que seja covariante, obtemos uma quantidade que é duas vezes o esperado. O erro está no fato de, mesmo no caso abeliano, os campos "fantasmas" (ghosts) de Faddeev-Popov contribuírem para a função de partição. A contribuição é tal que anula exatamente a parte vinda dos dois campos extras (longitudinal e tipo tempo). É uma surpresa que os campos de Faddeev-Popov sejam importantes mesmo no caso abeliano, pois ao contrário, a temperatura nula, a contribuição vinda destes campos fornece apenas uma constante multiplicativa ao funcional gerador, e podem ser ignorados. Aqui os termos são importantes e dão uma contribuição que cancela exatamente a dos graus de liberdade não físicos. A função de partição $Z$ deve ser definida como $\operatorname{Tr} \mathrm{e}^{-\beta \hat{H}}$ somente em um gauge que represente uma situação física, a fim de contarmos os graus de liberdade corretamente.

$\mathrm{O}$ que queremos dizer com toda esta explicação é que a lagrangeana para o campo de gauge deve ser modificada para

$$
\mathscr{L}=-\frac{1}{4} F_{\mu \nu} F^{\mu \nu}-\frac{1}{2 \xi}\left(\partial_{\mu} A^{\mu}\right)^{2}+\partial_{\mu} \bar{C} \partial^{\mu} C
$$

onde $C$ e $\bar{C}$ representam os campos de Faddeev-Popov. Desta forma, a função de partição que devemos tomar como ponto de partida é

$$
Z=[N(\beta)]^{2} \int_{\text {period }} D A^{\mu} D \bar{C} D C \exp \int \mathrm{d}^{4} \bar{x}\left(-\frac{1}{4} \bar{F}_{\mu \nu} \bar{F}^{\mu \nu}-\frac{1}{2 \xi}\left(\bar{\partial}_{\mu} A^{\mu}\right)^{2}+\bar{\partial}_{\mu} \bar{C} \bar{\partial}^{\mu} C\right)
$$

escrita em termos da coordenada $\bar{x}$. Agora o tratamento é o mesmo do caso anterior. Podemos reescrever (1.78) como

$$
\begin{aligned}
Z= & {[N(\beta)]^{2} \int_{\text {period }} D A^{\mu} \exp \left(-\frac{1}{2} \int \mathrm{d}^{4} \bar{x}^{\prime} \int \mathrm{d}^{4} \bar{x} A_{\mu}\left(\bar{x}^{\prime}\right) B^{\mu \nu}\left(\bar{x}^{\prime}, \bar{x}\right) A_{\nu}(\bar{x})\right) } \\
& \times \int_{\text {period }} D \bar{C} D C \exp \left(-\int \mathrm{d}^{4} \bar{x}^{\prime} \int \mathrm{d}^{4} \bar{x} \bar{C}\left(\bar{x}^{\prime}\right) G\left(\bar{x}^{\prime}, \bar{x}\right) C(\bar{x})\right)
\end{aligned}
$$

onde

$$
\begin{aligned}
B^{\mu \nu}\left(\bar{x}^{\prime}, \bar{x}\right) & =\left[\eta^{\mu \nu} \bar{\partial}_{\rho}^{\prime} \bar{\partial}^{\rho}-(1-1 / \xi) \bar{\partial}_{\nu}^{\prime} \bar{\partial}^{\mu}\right] \delta\left(\bar{x}^{\prime}-\bar{x}\right) \\
G\left(\bar{x}^{\prime}, \bar{x}\right) & =\bar{\partial}_{\rho}^{\prime} \bar{\partial}^{\rho} \delta\left(\bar{x}^{\prime}-\bar{x}\right)
\end{aligned}
$$

Usando a representação (1.65) para função delta, podemos escrever $B$ e $G$ como transformadas de Fourier

$$
\begin{aligned}
B^{\mu \nu}\left(\bar{x}^{\prime}, \bar{x}\right) & =\frac{1}{\beta} \sum_{n} \int \frac{\mathrm{d}^{3} p}{(2 \pi)^{3}} \mathrm{e}^{-i \bar{p} \cdot\left(\bar{x}^{\prime}-\bar{x}\right)}\left[\bar{p}^{2}\left(\eta^{\mu \nu}-\frac{\bar{p}^{\mu} \bar{p}^{\nu}}{\bar{p}^{2}}\right)+\frac{\bar{p}^{2}}{\xi} \frac{\bar{p}^{\mu} \bar{p}^{\nu}}{\bar{p}^{2}}\right] \\
G\left(\bar{x}^{\prime}, \bar{x}\right) & =\frac{1}{\beta} \sum_{n} \int \frac{\mathrm{d}^{3} p}{(2 \pi)^{3}} \mathrm{e}^{-i \bar{p} \cdot\left(\bar{x}^{\prime}-\bar{x}\right)} \bar{p}^{2}
\end{aligned}
$$


de forma que obtemos

$$
Z=[N(\beta)]^{2} \exp \left(-\frac{1}{2} \operatorname{Tr} \ln B\right) \exp (\operatorname{Tr} \ln G)
$$

O traço deve ser tomado sobre $\bar{x}$ e sobre os índices de Lorentz, e obtemos (termos independentes da temperatura foram desprezados)

$$
\begin{aligned}
& \operatorname{Tr} \ln B=\int \mathrm{d}^{3} x \sum_{n} \int \frac{\mathrm{d}^{3} p}{(2 \pi)^{3}} 4 \ln \left(\omega_{n}^{2}+\vec{p}^{2}\right) \\
& \operatorname{Tr} \ln G=\int \mathrm{d}^{3} x \sum_{n} \int \frac{\mathrm{d}^{3} p}{(2 \pi)^{3}} \ln \left(\omega_{n}^{2}+\vec{p}^{2}\right) .
\end{aligned}
$$

onde $\omega_{n}=2 \pi n / \beta$ é a freqüência de Matsubara para bósons. Obtemos assim para a função de partição

$$
Z=[N(\beta)]^{2} \exp \left(-\frac{1}{2} \int \mathrm{d}^{3} x \sum_{n} \int \frac{\mathrm{d}^{3} p}{(2 \pi)^{3}} 2 \ln \left(\omega_{n}^{2}+\vec{p}^{2}\right)\right)
$$

de maneira que, usando (1.69) e os resultados do Apêndice B.2,

$$
\ln Z=-2 \int \mathrm{d}^{3} x \int \frac{\mathrm{d}^{3} p}{(2 \pi)^{3}}\left\{\frac{\beta}{2} \sqrt{\vec{p}^{2}}+\ln \left[1-\exp \left(-\beta \sqrt{\vec{p}^{2}}\right)\right]+C_{2}(\beta)\right\}+\ln [N(\beta)]^{2},
$$

onde $C_{2}(\beta)$ é uma constante dependente da temperatura mas independente de $\vec{p}$, de maneira que sua integração fornece uma contribuição infinita para $\ln Z$. Mas também pode ser mostrado [14] que este termo cancela exatamente o termo $\ln [N(\beta)]^{2}$ em (1.85).

Voltando então à função de partição, podemos escrever para a energia livre

$$
F=-\frac{1}{\beta} \ln Z=2 \int \mathrm{d}^{3} x \int \frac{\mathrm{d}^{3} p}{(2 \pi)^{3}}\left(\frac{1}{2} \sqrt{\vec{p}^{2}}+\frac{1}{\beta} \ln \left[1-\exp \left(-\beta \sqrt{\vec{p}^{2}}\right)\right]\right) .
$$

O primeiro termo dá uma contribuição infinita que deve ser desconsiderada, pois representa a energia do vácuo. Desta forma obtemos para a densidade de energia livre

$$
\begin{aligned}
\frac{F}{V} & =2 \int \frac{\mathrm{d}^{3} p}{(2 \pi)^{3}} \frac{1}{\beta} \ln \left[1-\exp \left(-\beta \sqrt{\vec{p}^{2}}\right)\right] \\
& =2 \times 4 \pi \frac{1}{\beta} \frac{1}{(2 \pi)^{3}} \int_{0}^{\infty} p^{2} \ln \left(1-\mathrm{e}^{-p \beta}\right) \mathrm{d} p \\
& =8 \pi \frac{1}{\beta} \frac{1}{(2 \pi)^{3}} \frac{-\pi^{4}}{45 \beta^{3}} \\
& =-2 \frac{\pi^{2} T^{4}}{90}
\end{aligned}
$$


Com isto, a pressão, a densidade de entropia e a densidade de energia são

$$
\begin{aligned}
P & =2 \frac{\pi^{2} T^{4}}{90}, \\
\frac{S}{V} & =\frac{4 \pi^{2} T^{3}}{45}, \\
\frac{E}{V} & =\frac{2 \pi^{2} T^{4}}{30},
\end{aligned}
$$

que é exatamente o dobro do caso de um campo escalar, que é o esperado, já que o campo vetorial $A^{\mu}$ só tem dois graus de liberdade físicos. Além disto, tanto aqui quanto no caso de férmions, é satisfeita a equação de estado

$$
P=\frac{1}{3} E / V
$$

como deve ser para o caso de um gás ideal relativístico.

\subsubsection{Auto-energia do fóton}

Vamos ver agora como fica o cálculo de um diagrama de 1-loop para o caso a temperatura finita. Para isso vamos aplicar os resultados anteriores para um caso específico, que é o da auto-energia do fóton, representado pelo diagrama da Figura 1.3. Aqui o quadrimomento do fóton será representado por $k$, portanto $q \rightarrow k$ naquela figura. A auto-energia pode ser representada em termos do propagador livre $D_{0}^{\mu \nu}$ e do propagador exato $D^{\mu \nu}$ por

$$
\Pi_{\mu \nu}=D_{\mu \nu}^{-1}-D_{0 \mu \nu}^{-1}
$$

onde o propagador está relacionado ao seu inverso por

$$
D^{\mu \alpha} D_{\alpha \nu}^{-1}=\eta_{\nu}^{\mu}
$$

Além disso, o propagador e a auto-energia devem satisfazer a alguns vínculos. Denotando $k^{\mu}$ o quadrimomento do fóton, a conservação da corrente requer que $\Pi_{\mu \nu}$ seja transversal ao quadrimomento do fóton

$$
k^{\mu} \Pi_{\mu \nu}=0
$$

e a invariância de gauge requer que

$$
k^{\mu} k^{\nu} D_{\mu \nu}=\xi
$$

onde $\xi$ especifica uma escolha de gauge. No caso a temperatura nula, o tensor de auto-energia do fóton $\Pi^{\mu \nu}$ depende somente de $\eta^{\mu \nu}$ e do momento externo $k^{\mu}$ do fóton, conforme (1.16). Agora, a temperatura finita, precisamos de mais um quadrivetor para especificar o sistema corretamente, e este quadrivetor é 
representado por $u^{\mu}$. Ele é o quadrivetor que representa a velocidade do reservatório térmico onde o sistema se encontra, e o caso $u_{\mu}=(1,0,0,0)$ especifica o sistema de repouso desse reservatório. Em cálculos específicos podemos sempre adotar este sistema como o de referência, o que indica que estamos no sistema do reservatório térmico. Dito isto, a auto-energia, o propagador e seu inverso são tensores simétricos de segunda ordem formados pelas estruturas acima. Para que satisfaça as relações (1.92)-(1.95), pode-se mostrar que eles devem ter as formas

$$
\begin{gathered}
\Pi^{\mu \nu}(k)=\Pi_{\mathrm{T}} P_{\mathrm{T}}^{\mu \nu}+\Pi_{\mathrm{L}} P_{\mathrm{L}}^{\mu \nu}, \\
D^{\mu \nu}=\frac{1}{k^{2}-\Pi_{\mathrm{T}}} P_{\mathrm{T}}^{\mu \nu}+\frac{1}{k^{2}-\Pi_{\mathrm{L}}} P_{\mathrm{L}}^{\mu \nu}+\frac{\xi}{k^{2}} \frac{k^{\mu} k^{\nu}}{k^{2}}, \\
\left(D^{-1}\right)^{\mu \nu}=\left(k^{2}-\Pi_{\mathrm{T}}\right) P_{\mathrm{T}}^{\mu \nu}+\left(k^{2}-\Pi_{\mathrm{L}}\right) P_{\mathrm{L}}^{\mu \nu}+\frac{k^{\mu} k^{\nu}}{\xi},
\end{gathered}
$$

onde $\Pi_{\mathrm{T}}$ e $\Pi_{\mathrm{L}}$ são funções escalares que podem ser determinadas. Vamos nos restringir ${ }^{13}$ ao sistema de referência do reservatório térmico, $u^{\mu}=(1,0,0,0)$ e ao limite estático, $k_{0}=0$. A parte tensorial fica por conta dos operadores

$$
\begin{gathered}
P_{\mathrm{L}}^{\mu \nu}=u^{\mu} u^{\nu} \\
P_{\mathrm{T}}^{\mu \nu}=\eta^{\mu \nu}-u^{\mu} u^{\nu}-\frac{k^{\mu} k^{\nu}}{k^{2}},
\end{gathered}
$$

e satisfazem as seguintes propriedades:

$$
\begin{aligned}
P_{\mathrm{L}}^{\mu \nu} P_{\mathrm{L} \mu \nu} & =1, \\
P_{\mathrm{T}}^{\mu \nu} P_{\mathrm{T} \mu \nu} & =(d-2), \\
k_{\mu} P_{\mathrm{T}}^{\mu \nu} & =k_{\mu} P_{\mathrm{L}}^{\mu \nu}=0, \\
P_{\mathrm{L}}^{\mu \nu} P_{\mathrm{T} \mu \nu} & =0,
\end{aligned}
$$

onde $d$ é a dimensão do espaço-tempo e vem da contração $\eta^{\mu \nu} \eta_{\mu \nu}=d$. Usando (1.96)-(1.101) pode-se verificar que as relações (1.93)-(1.95) são de fato satisfeitas. Com estas relações os coeficientes $\Pi_{\mathrm{L}}$ e $\Pi_{\mathrm{T}}$ podem ser determinados facilmente. Basta contrair os dois lados de (1.96) por $P_{\mathrm{L}}^{\mu \nu}$ e $P_{\mathrm{T}}^{\mu \nu}$, e usando as

\footnotetext{
${ }^{13}$ Para o caso geral ver págs. $70-71$ da ref. [11] ou págs. $118-119$ de [12].
} 
relações (1.101) obtemos

$$
\begin{aligned}
& P_{\mathrm{L}}^{\mu \nu} \Pi_{\mu \nu}=\Pi_{\mathrm{L}} \\
& P_{\mathrm{T}}^{\mu \nu} \Pi_{\mu \nu}=(d-2) \Pi_{\mathrm{T}} .
\end{aligned}
$$

Vamos então calcular $\Pi^{\mu \nu}$.

Usando as regras de Feynman a temperatura finita, a expressão para o diagrama que representa $\Pi^{\mu \nu}$ é, conforme Figura 1.3,

$$
\Pi^{\mu \nu}(k)=-e^{2} T \sum_{l} \int \frac{\mathrm{d}^{3} p}{(2 \pi)^{3}} \operatorname{tr}\left(\gamma^{\mu} \frac{1}{\not p+\not k-m} \gamma^{\nu} \frac{1}{\not p-m}\right),
$$

onde $p^{0}=(2 l+1) \pi T i$ está associada à freqüência para os férmions e $k^{0}=2 n \pi T i$ à freqüência para o fóton. Pode-se mostrar que sempre podemos escrever o tensor de auto-energia como a soma de uma parte de vácuo (independente de $T$ ) e uma parte de matéria (dependente de $T$ ), na forma

$$
\Pi^{\mu \nu}=\Pi_{(v a c)}^{\mu \nu}+\Pi_{(m a t)}^{\mu \nu}
$$

de tal maneira que

$$
\Pi_{(v a c)}^{\mu \nu}=\lim _{\substack{T \rightarrow 0 \\ \mu \rightarrow 0}} \Pi_{(m a t)}^{\mu \nu}
$$

A parte de vácuo é exatamente igual àquela que foi calculada em (1.14), como era de se esperar. A parte de matéria não pode ser calculada exatamente, mas alguns de seus limites e várias outras aplicações envolvendo esta expressão foram extensivamente estudas por J. I. Kapusta e vários outros autores, conforme referências $[11,12,15,16,17]$. Vamos discutir aqui apenas um dos limites da expressão (1.103), que é o limite estático a altas temperaturas ${ }^{14}$. Para obtermos este limite basta tomar o momento interno ao loop $p$ muito maior que o momento externo $k$ e a massa $m$, de forma de que a expressão (1.103) fica

$$
\begin{aligned}
\Pi^{\mu \nu} & =-e^{2} T \sum_{l} \int \frac{\mathrm{d}^{3} p}{(2 \pi)^{3}} \operatorname{tr}\left(\gamma^{\mu} \frac{1}{\not p} \gamma^{\nu} \frac{1}{\not p}\right) \\
& =-e^{2} T \sum_{l} \int \frac{\mathrm{d}^{3} p}{(2 \pi)^{3}} \operatorname{tr}\left(\gamma^{\mu} \frac{\not p}{p^{2}} \gamma^{\nu} \frac{\not p}{p^{2}}\right) \\
& =-e^{2} T \sum_{l} \int \frac{\mathrm{d}^{3} p}{(2 \pi)^{3}} \frac{p_{\alpha} p_{\beta}}{p^{4}} \operatorname{tr}\left(\gamma^{\mu} \gamma^{\alpha} \gamma^{\nu} \gamma^{\beta}\right) \\
& =-4 e^{2} T \sum_{l} \int \frac{\mathrm{d}^{3} p}{(2 \pi)^{3}}\left(\frac{2 p^{\mu} p^{\nu}}{p^{4}}-\frac{\eta^{\mu \nu}}{p^{2}}\right),
\end{aligned}
$$

\footnotetext{
${ }^{14} \mathrm{Na}$ literatura é comum a nomenclatura Hard thermal loop approximation, ou simplesmente limite HTL.
} 
onde foi usada a propriedade do traço

$$
\operatorname{tr}\left(\gamma^{\mu} \gamma^{\alpha} \gamma^{\nu} \gamma^{\beta}\right)=4\left(\eta^{\mu \alpha} \eta^{\nu \beta}+\eta^{\mu \beta} \eta^{\nu \alpha}-\eta^{\mu \nu} \eta^{\alpha \beta}\right)
$$

Usando (1.102) podemos determinar os coeficientes $\Pi_{\mathrm{L}}$ e $\Pi_{\mathrm{T}}$. No sistema de repouso do reservatório, temos

$$
\begin{aligned}
\Pi_{\mathrm{L}} & =\Pi^{00} \\
& =-4 e^{2} T \sum_{l} \int \frac{\mathrm{d}^{3} p}{(2 \pi)^{3}}\left(\frac{2 p^{0} p^{0}}{p^{4}}-\frac{\eta^{00}}{p^{2}}\right) \\
& =-4 e^{2} T \sum_{l} \int \frac{\mathrm{d}^{3} p}{(2 \pi)^{3}}\left(\frac{1}{p^{2}}+\frac{2|\vec{p}|^{2}}{p^{4}}\right) \\
& =-4 e^{2} \int \frac{\mathrm{d}^{3} p}{(2 \pi)^{3}} T \sum_{l}\left(\frac{1}{[(2 l+1) \pi T i]^{2}-\vec{p}^{2}}+\frac{2|\vec{p}|^{2}}{\left([(2 l+1) \pi T i]^{2}-\vec{p}^{2}\right)^{2}}\right) \\
& =-4 e^{2} \int \frac{\mathrm{d}^{3} p}{(2 \pi)^{3}}\left[\frac{n_{F}(|\vec{p}|)}{|\vec{p}|}+2|\vec{p}|^{2}\left(\frac{n_{F}^{\prime}(|\vec{p}|)}{2|\vec{p}|^{2}}-\frac{n_{F}(|\vec{p}|)}{2|\vec{p}|^{3}}\right)\right] \\
& =-4 e^{2} \int \frac{\mathrm{d}^{3} p}{(2 \pi)^{3}} n_{F}^{\prime}(|\vec{p}|) \\
& =-\frac{16 \pi e^{2}}{(2 \pi)^{3}} \int_{0}^{\infty} p^{2}\left(\frac{1}{\mathrm{e}^{\beta p}+1}\right)^{\prime} \mathrm{d} p \\
& =\frac{e^{2} T^{2}}{3} .
\end{aligned}
$$

A primeira soma sobre as freqüências fermiônicas foi feita usando os resultados do Apêndice B.3, eq. (B.25). A segunda soma pode ser feita a partir da primeira, derivando com relação a $|\vec{p}|$ dos dois lados, e $n_{F}(|\vec{p}|)$ é a função distribuição de Fermi-Dirac, eq. (1.49). A última integral pode ser feita por integração por partes.

Procedendo de forma análoga obtemos

$$
\begin{aligned}
\Pi_{\mathrm{T}} & =\frac{1}{2} P_{\mathrm{T}}^{\mu \nu} \Pi_{\mu \nu} \\
& =0 .
\end{aligned}
$$

Vamos ver a seguir uma aplicação destes resultados. 


\subsubsection{Blindagem dos campos elétrico e magnético em um plasma}

Quando a temperatura de um gás é elevada a valores muito altos, os átomos que compõem este gás se ionizam facilmente, deixando os elétrons livres, e isto caracteriza um estado chamado de plasma eletromagnético. Se uma carga positiva $Q$ for introduzida neste plasma, os elétrons rapidamente são atraídos pela carga positiva, distribuindo-se em torno dela de maneira a neutralizá-la. A este fenômeno dá-se o nome de "blindagem". O campo elétrico produzido pela carga $Q$ perde a intensidade a medida que afastamos de seu centro, e o potencial criado por essa carga tem a forma

$$
V(r)=\frac{Q}{r} \mathrm{e}^{-r / r_{D}}
$$

onde $r_{D}$ caracteriza o "raio de blindagem" da carga, ou seja, a distância a partir da qual o potencial é praticamente nulo. Este comprimento é conhecido como "raio de Debye", e seu inverso caracteriza a "massa elétrica" do meio, $m_{e l}=1 / r_{D}$. Um gás ionizado é considerado um plasma se o raio de Debye $r_{D}$ for pequeno em relação a outras dimensões físicas de interesse. Em uma escala maior que $r_{D}$, os elétrons tendem a cooperar de maneira a neutralizar um excesso de cargas positivas em um determinado ponto. É esta resposta coletiva às flutuações de carga que dá origem às oscilações do plasma em larga escala.

As massas elétrica e magnética estão relacionadas ao tensor de auto-energia do fóton. Elas são definidas como sendo os pólos $\Pi_{L}$ e $\Pi_{T}$ presentes no propagador do fóton, equação (1.97), no chamado limite estático, $k_{0}=0$. Lembre-se que $k$ é o quadrimomento do fóton, e o fóton é o verdadeiro responsável pela "transmissão" dos campos eletromagnéticos. Quando $k^{\mu}=(0, \vec{k})$, significa que a freqüência associada aos campos eletromagnéticos é nula, ou seja, estamos diante de campos estáticos, o que caracteriza o limite estático. A massa elétrica corresponde ao pólo da parte longitudinal do propagador e a massa magnética ao pólo da parte transversal. Elas são definidas por

$$
\begin{gathered}
m_{\mathrm{el}}^{2} \equiv \Pi_{\mathrm{L}}\left(k_{0}=0, \vec{k}\right), \\
m_{\mathrm{mag}}^{2} \equiv \Pi_{\mathrm{T}}\left(k_{0}=0, \vec{k}\right) .
\end{gathered}
$$

Usando os resultados (1.108) e (1.109) obtemos no limite estático a altas temperaturas

$$
\begin{aligned}
m_{\mathrm{el}}^{2} & =\Pi_{\mathrm{L}}\left(k_{0}=0, \vec{k} \rightarrow 0\right)=\frac{e^{2} T^{2}}{3}, \\
m_{\mathrm{mag}}^{2} & =0
\end{aligned}
$$

Portanto, campos elétricos estáticos em um plasma a altas temperaturas são blindados, e campos magnéticos não.

A massa elétrica pode ser entendida como uma massa efetiva adquirida pelo fóton dentro do plasma. Se o fóton adquire uma massa, o alcance de sua interação não é mais infinito como antes, o que corresponde 
a dizer que o campo elétrico gerado pela carga tem um alcance finito, além do qual ele não atua mais. É a blindagem de que estamos falando. Quanto maior a temperatura, maior a massa elétrica, e portanto menor o raio de blindagem. Isto significa que o alcance do campo elétrico vai diminuindo com o aumento da temperatura, até o limite em que a carga passa se comportar como sendo neutra, que é quando $T \rightarrow \infty$.

Como já dissemos no início desta seção, classicamente este fenômeno é conhecido na física de plasmas, e a visão clássica é a de que há uma polarização das cargas, formando dipolos elétricos em torno da carga central, o que provoca sua blindagem. Isto pode ser melhor visualizado na Figura 1.12. Cargas positivas se

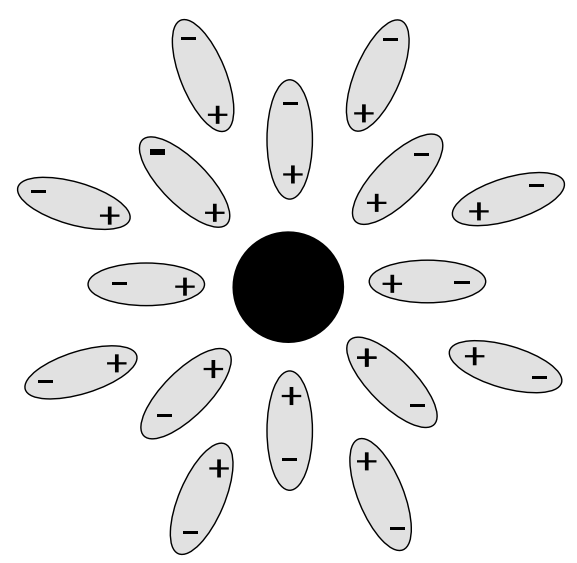

Figura 1.12: Blindagem do campo elétrico produzido por uma carga $-Z e$ no centro.

aproximam da carga central, e cargas negativas são repelidas. Sucessivamente outras cargas positivas são atraídas e negativas repelidas, levando a uma quase neutralidade do ponto de vista macroscópico. Do ponto de vista quântico podemos representar a blindagem da carga conforme a Figura 1.13. Os fótons, que são

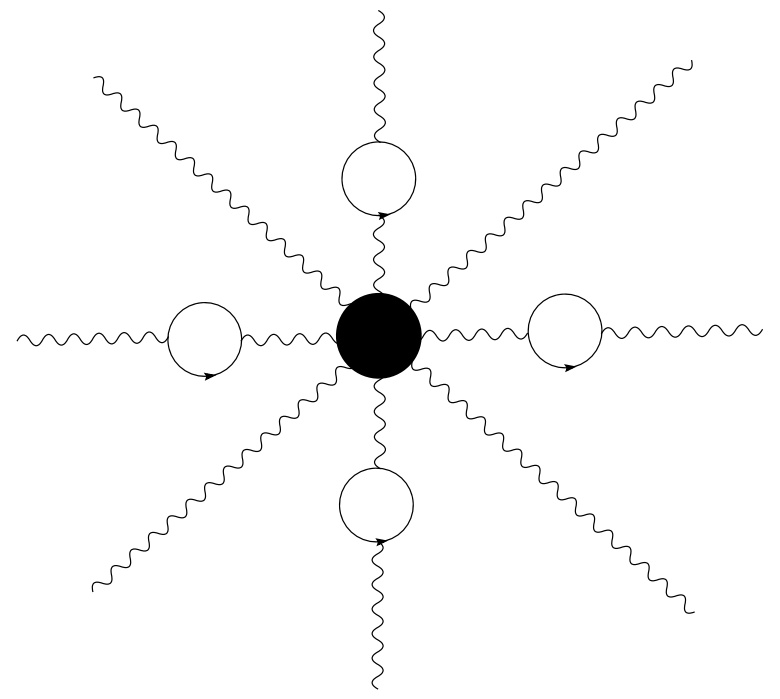

Figura 1.13: Visão quântica da blindagem do campo elétrico de uma carga $Q$ no centro. 
os responsáveis pela interação eletromagnética da carga central com os íons do plasma, podem, segundo a QED, sofrer os processos representados pelos diagramas de 1-loop. Isto acarreta em uma geração efetiva de massa ao fóton, que portanto deixa de ter o seu alcance infinito, ou seja, o campo elétrico associado a este fóton tem uma ação finita, deixando de agir a partir de uma certa distância, o que caracteriza a blindagem da carga.

Nesta seção discutimos como a presença da temperatura pode modificar os cálculos da teoria de campos usual da seção anterior. Cálculos a temperatura finita nos dão a possibilidade de obter novas quantidades de interesse de um sistema, como a função de partição por exemplo e todas as quantidades físicas que dela derivam. Também vimos que o tensor de auto-energia do fóton (1.103) a altas temperaturas é dado por,

$$
\Pi^{\mu \nu}=\Pi_{(v a c)}^{\mu \nu}+\frac{e^{2} T^{2}}{3} u^{\mu} u^{\nu}
$$

onde a parte de vácuo foi calculada na seção 1.1.4. Observe que o método da teoria de campos a temperatura finita é mais geral do que a teoria de campos usual no sentido de que ela fornece todos os resultados a temperatura nula acrescido de uma correção devido à presença da temperatura. Da expressão acima fica evidente que a seção de choque de uma reação se modifica se ela acontecer imersa em um banho térmico, e quanto mais alta a temperatura, maior será a contribuição. Mas também temos que lembrar que as expressões anteriores foram obtidas no limite de altas temperaturas, ou seja, quando a temperatura é muito maior que a massa das partículas envolvidas. Para uma partícula como o elétron por exemplo, a temperatura correspondente à sua massa de repouso é da ordem de $10^{9} \mathrm{~K}$, portanto uma temperatura muito alta, lembrando que uma estrela típica como sol tem uma temperatura superficial da ordem de $10^{4} \mathrm{~K}$. No entanto existem outros lugares onde temperaturas muito altas devem ocorrer, como em explosão de supernovas, núcleos galáticos, e certamente nos primórdios do universo. Por isso o interesse de se entender corretamente como incluir efeitos de temperatura em teoria de campos. 



\section{Capítulo 2}

\section{Teorias Não-Comutativas}

O advento da teoria de campos a partir da década de 1930 foi acompanhado por uma busca incansável de mecanismos que pudessem dar conta de remover corretamente os inúmeros infinitos a que a teoria estava sujeita $^{1}$. As chamadas divergências ultravioletas e infravermelhas só foram completamente entendidas com o desenvolvimento do programa de renormalização, no entanto outras alternativas foram propostas. Uma delas foi sugerida por um dos fundadores da mecânica quântica e da eletrodinâmica quântica, W. Heisenberg. Ele propôs que uma estrutura não-comutativa do espaço em escalas de comprimento muito pequenas era capaz de introduzir um parâmetro de corte (cutoff) ultravioleta, de tal forma a se evitar o aparecimento das divergências. Snyder [18] foi o primeiro a formular esta idéia de maneira consistente.

Evidências mais concretas da não-comutatividade do espaço-tempo vêm do limite de baixas energias da teoria de cordas, no momento a melhor teoria candidata a incorporar efeitos quânticos da gravidade. Como as cordas têm uma escala de comprimento finito da ordem do comprimento de Planck $l_{P}$, não é possível observar distâncias menores que $l_{P}$.

Outra motivação para se aceitar com mais naturalidade a não-comutatividade do espaço-tempo em escalas de comprimento muito pequenas vem da gravitação. Em escalas da ordem do comprimento de Planck o conceito de medida perde o seu significado. Quanto mais tentamos localizar uma partícula, por exemplo pelo espalhamento de um fóton, menor deve ser o seu comprimento de onda, e conseqüentemente maior a energia associada a ela. Desta forma mais energia está sendo transferida ao sistema, o que significa uma contribuição para o campo gravitacional local através das equações de Einstein da relatividade geral, gerando uma curvatura do espaço e interferindo nos sinais luminosos que dariam informação sobre a medida.

De fato, todos estes efeitos só têm validade em escalas de energia muitíssimo altas, onde a física provavelmente deve seguir outras leis que não as que conhecemos. Desta forma devemos considerar a não-comutatividade do espaço-tempo como uma teoria efetiva, capaz de levar em conta a existência de um comprimento mínimo. Além disso no limite de baixas energias, ou $\theta \rightarrow 0$, ela recai nas teorias usuais que conhecemos bem.

A idéia por trás da não-comutatividade do espaço-tempo é muito próxima à que encontramos na

\footnotetext{
${ }^{1}$ Um exemplo destes infinitos apareceu no cálculo do tensor de auto-energia do fóton do capítulo anterior.
} 
mecânica quântica, onde o espaço de fases quântico é obtido pela troca das variáveis canônicas de posição $x_{i}$ e momento $p_{j}$ por operadores hermiteanos $\hat{x}_{i}, \hat{p}_{j}$ que obedecem às relações de comutação de Heisenberg $\left[\hat{x}_{i}, \hat{p}_{j}\right]=i \hbar \delta_{i j}$. A esta relação de comutação sabemos estar associada uma relação de incerteza na medida da posição e do momento de uma partícula, expressa na forma $\Delta x \Delta p_{x} \geq \hbar / 2$. Da mesma maneira o espaço-tempo não-comutativo é definido pela troca das coordenadas do espaço-tempo usual $x^{\mu}$ por geradores hermiteanos $\hat{x}^{\mu}$ de uma álgebra não-comutativa que obedeça à relação de comutação

$$
\left[\hat{x}^{\mu}, \hat{x}^{\nu}\right]=i \theta^{\mu \nu}
$$

onde, no caso mais simples, $\theta^{\mu \nu}$ é um tensor real, constante e anti-simétrico, com dimensão de comprimento ao quadrado. A relação de incerteza associada é do tipo $\Delta x^{\mu} \Delta x^{\nu} \geq \theta / 2$, onde $\theta \equiv\left|\theta^{\mu \nu}\right|$ deve ter dimensão de comprimento ao quadrado. Devido a esta relação de incerteza, nesta escala um ponto do espaço-tempo deve ser trocado por uma "célula" com dimensão de área da ordem de $\theta$. Para descrever fenômenos nesta escala devemos então utilizar uma álgebra que seja não-comutativa. Desta forma, a teoria de campos é válida apenas até uma escala de comprimento da ordem de $\sqrt{\theta}$, abaixo da qual uma nova teoria deve ser adotada, por exemplo a teoria de cordas. Portanto as divergências ultravioletas da teoria não devem existir, pois a um comprimento mínimo está associado um momento máximo, que deve ser tomado como o parâmetro de corte da integração.

Para termos uma idéia da ordem de grandeza de $\sqrt{\theta}$, vamos considerar que de fato a não-comutatividade é uma teoria efetiva que segue do limite de baixas energias da teoria de cordas. Se a escala de comprimento da teoria de cordas é o comprimento de Planck, $l_{P} \sim 10^{-33} \mathrm{~cm}$, a não-comutatividade deve ter uma escala muito maior que $l_{P}$ mas ainda assim muito menor que a escala quântica, que é da ordem de $1 \mathrm{fm}$ $\sim 10^{-13} \mathrm{~cm}$. De fato, limites experimentais $[19,20]$ impõem um valor de $\sqrt{\theta} \leq 10^{-18} \mathrm{~cm}$, que corresponde em energia ${ }^{2}$ a $\theta \sim(10 \mathrm{TeV})^{-2}$.

Neste capítulo vamos apresentar brevemente uma motivação para o estudo da não-comutatividade entre as coordenadas que vem da física clássica. Os resultados apresentados na seção 2.1.2 foram publicados nas referências $[72,73]$. Em seguida apresentaremos a formulação do ponto de vista da teoria de campos e terminamos com uma breve apresentação das regras de Feynman para o caso da eletrodinâmica quântica não-comutativa, que será útil no capítulo seguinte.

\subsection{Não-comutatividade na física clássica}

Nesta seção faremos uma breve exposição de como a não-comutatividade das coordenadas aparece já na mecânica clássica, no conhecido problema de Landau, onde uma partícula carregada sujeita a um campo magnético forte tem seu movimento restrito ao plano perpendicular ao campo. A quantização deste sistema foi estudada por Landau [21] e rediscutida por inúmeros autores [22].

\footnotetext{
${ }^{2}$ Estamos usando $1 \mathrm{GeV}^{-1} \sim 10^{-14} \mathrm{~cm}$.
} 


\subsubsection{Partícula carregada em um campo magnético intenso e uniforme}

Considere uma partícula de massa $m$ e carga $e$ movendo-se no plano $x-y$ sob ação de um campo magnético constante $B$ na direção $z$ e sujeita a um potencial escalar externo $-V(x, y)$. Nosso objetivo é mostrar que para este sistema clássico, quando o campo magnético constante $B$ é muito intenso, a quantização do sistema leva a uma não-comutatividade das coordenadas, ou $[x, y] \neq 0$. Este problema foi extensivamente estudado por Jackiw e colaboradores, conforme referências [23, 24, 25, 26].

A lagrangeana para este sistema deve ter a forma geral

$$
L=\frac{1}{2} m \vec{v}^{2}+\frac{e}{c} \vec{v} \cdot \vec{A}(x, y)-V(x, y)
$$

onde $\vec{v}$ é a velocidade da partícula, $\vec{v}=\left(v_{x}, v_{y}\right) \equiv(\dot{x}, \dot{y}), \vec{A}$ é o potencial vetor eletromagnético e $V$ um potencial escalar qualquer, da forma

$$
V(x, y)=a x^{n}+b y^{n},
$$

para $a$ e $b$ constantes. Não há perda de generalidade se tomarmos o potencial $V$ como sendo o de um oscilador harmônico, portanto vamos nos restringir a

$$
V(x, y)=\frac{1}{2} k\left(x^{2}+y^{2}\right)
$$

com $k$ constante. A fim de que o campo magnético seja constante na direção $z$, vamos escolher ${ }^{3}$

$$
\vec{A}(x, y)=\left(-\frac{B}{2} y, \frac{B}{2} x\right),
$$

de forma que $\vec{\nabla} \times \vec{A}=B \hat{z}$. Com isto, a lagrangeana pode ser escrita como

$$
L=\frac{1}{2} m\left(\dot{x}^{2}+\dot{y}^{2}\right)+\frac{e B}{2 c}(x \dot{y}-y \dot{x})-\frac{1}{2} k\left(x^{2}+y^{2}\right) .
$$

As equações de movimento deste sistema seguem diretamente das equações de Euler-Lagrange (ver Apêndice A.1 para uma breve revisão), e são dadas por

$$
m \ddot{x}=\frac{e B}{c} \dot{y}-k x, \quad m \ddot{y}=-\frac{e B}{c} \dot{x}-k y .
$$

Os momentos canonicamente conjugados a $x$ e $y$ são

$$
P_{x}=\frac{\partial L}{\partial \dot{x}}=m \dot{x}-\frac{e B}{2 c} y, \quad P_{y}=\frac{\partial L}{\partial \dot{y}}=m \dot{y}+\frac{e B}{2 c} x,
$$

e como sabemos, as coordenadas $x, y$ e os momentos conjugados $P_{x}, P_{y}$ satisfazem as relações dos colche-

\footnotetext{
${ }^{3}$ Poderíamos fazer outra escolha, por exemplo $\vec{A}=(0, B x)$, pois a teoria é invariante por uma transformação de gauge.
} 
tes $^{4}$ de Poisson,

$$
\{x, y\}=\left\{P_{x}, P_{y}\right\}=\left\{x, P_{y}\right\}=\left\{y, P_{x}\right\}=0, \quad\left\{x, P_{x}\right\}=\left\{y, P_{y}\right\}=1
$$

A hamiltoniana pode ser obtida facilmente por meio de $H=\vec{P} \cdot \vec{v}-L$, conforme (A.3), de onde seguem as equações de Hamilton do movimento

$$
\dot{x}=\{x, H\}=\frac{1}{m}\left(P_{x}+\frac{e B}{2 c} y\right), \quad \dot{y}=\{y, H\}=\frac{1}{m}\left(P_{y}-\frac{e B}{2 c} x\right),
$$

que são exatamente equivalentes àquelas da eq. (2.7), como deve ser.

Agora vamos tomar o limite em que o campo magnético $B$ é muito intenso. Uma forma de implementar isso é tomar o limite $m \rightarrow 0$ na lagrangeana, de forma que toda a cinética fica por conta da ação do campo magnético, sob influência do potencial $V$, que vamos manter apenas por generalidade. A lagrangeana neste caso fica

$$
L_{0}=\frac{e B}{2 c}(x \dot{y}-y \dot{x})-\frac{1}{2} k\left(x^{2}+y^{2}\right) .
$$

e as equações de movimento (2.6) se reduzem a

$$
\dot{x}=-\frac{k c}{e B} y, \quad \dot{y}=\frac{k c}{e B} x .
$$

A hamiltoniana fica dada simplesmente por

$$
H_{0}=V(x, y)=\frac{1}{2} k\left(x^{2}+y^{2}\right)
$$

mas agora aparece um problema. As equações de Hamilton (2.9) não podem ser obtidas através do comutador de Poisson, pois de acordo com as relações (2.8)

$$
\begin{aligned}
\dot{x}=\left\{x, H_{0}\right\} & =\left\{x, \frac{1}{2} k\left(x^{2}+y^{2}\right)\right\} \\
& =k y\{x, y\} \\
& =0,
\end{aligned}
$$

em desacordo com (2.11). Uma maneira de resolver este problema é propondo que

$$
\{x, y\}=-\frac{c}{e B}
$$

de forma a restabelecer os resultados (2.11). Mas este comutador não deve ser o de Poisson, pois este sabemos que é nulo. Admitindo por um momento a existência deste novo comutador, a extensão quântica

\footnotetext{
${ }^{4}$ Usaremos a notação $\{$,$\} para colchetes de Poisson ou comutador de Poisson, \{,\}_{\text {D }}$ para colchetes de Dirac ou comutador de Dirac, e [, ] para o comutador quântico.
} 
de um sistema clássico é feita por meio da substituição

$$
\{f, g\}=\frac{1}{i \hbar}[\hat{f}, \hat{g}]
$$

onde $f, g$ são funções clássicas do lado esquerdo e operadores quânticos do lado direito. Quando aplicamos ao nosso problema obtemos

$$
[x, y]=-\frac{i \hbar c}{e B}
$$

Desta forma podemos dizer que as coordenadas não comutam, sendo a intensidade do parâmetro de nãocomutatividade proporcional ao inverso do campo magnético $B$ que atua no sistema.

No entanto existe uma maneira mais rigorosa de se obter este resultado. Ela foi proposta por Dirac para quantizar sistemas sujeitos a vínculos [27], que é exatamente quando os colchetes de Poisson não fornecem uma boa descrição para a quantização, que é o nosso caso, afinal $\{x, y\}=0$.

Vamos ver então como fica o problema por meio do método de Dirac. Segundo este método, quando o sistema está sujeito a vínculos, o colchetes de Poisson entre duas quantidades $Q_{1}$ e $Q_{2}$ deve ser generalizado para o chamado colchetes de Dirac

$$
\left\{Q_{1}, Q_{2}\right\}_{\mathrm{D}}=\left\{Q_{1}, Q_{2}\right\}-\left\{Q_{1}, \chi^{i}\right\} C_{i j}\left\{\chi^{j}, Q_{2}\right\}
$$

onde $\chi^{i}$ são os vínculos a que o sistema está sujeito, e $C_{i j}$ é obtido através de

$$
C_{i j}\left\{\chi^{j}, \chi^{k}\right\}=\delta_{i}^{k}
$$

onde $\delta_{i}^{k}$ é o delta de Kronecker. Vamos aplicar ao nosso problema. Temos dois vínculos, que aparecem quando impomos $m \rightarrow 0$ nas equações (2.7)

$$
\begin{aligned}
& \chi^{1} \equiv P_{x}+\frac{e B}{2 c} y=m \dot{x} \approx 0, \\
& \chi^{2} \equiv P_{y}-\frac{e B}{2 c} x=m \dot{y} \approx 0 .
\end{aligned}
$$

Estes vínculos são de segunda classe ${ }^{5}$, pois

$$
\left\{\chi^{1}, \chi^{2}\right\}=\left\{P_{x}+\frac{e B}{2 c} y, P_{y}-\frac{e B}{2 c} x\right\}=\frac{e B}{c} \neq 0,
$$

\footnotetext{
${ }^{5}$ Não vamos nos deter nas definições aqui. Para um tratamento rigoroso sugerimos consultar as referências [27, 28]. Apenas por completeza, vínculos de primeira classe possuem parênteses de Poisson nulo com qualquer outro vínculo, e vínculos de segunda classe são aqueles cujos parênteses de Poisson é diferente de zero com pelo menos um dos vínculos. Como regra geral, os vínculos devem ser escolhidos de maneira a maximizar os de primeira classe. Isto pode ser feito levando-se em conta que uma combinação linear de vínculos também é um vínculo.
} 
onde usamos os resultados (2.8). Os coeficientes $C_{12}$ e $C_{21}$ podem ser facilmente obtidos, por exemplo

$$
\begin{aligned}
C_{12}\left\{\chi^{2}, \chi^{k}\right\} & =\delta_{1}^{k} \\
C_{12}\left\{\chi^{2}, \chi^{1}\right\} & =1 \\
C_{12}\left(-\frac{e B}{c}\right) & =1 \\
C_{12}= & -\frac{c}{e B},
\end{aligned}
$$

e da mesma forma obtemos $C_{21}=\frac{c}{e B}$. Portanto, calculando os comutadores de Poisson de $x, y \operatorname{com} \chi^{1}, \chi^{2}$ obtemos

$$
\left\{x, \chi^{1}\right\}=1, \quad\left\{x, \chi^{2}\right\}=0, \quad\left\{\chi^{1}, y\right\}=0, \quad\left\{\chi^{2}, y\right\}=-1
$$

de forma que temos finalmente

$$
\begin{aligned}
\{x, y\}_{\mathrm{D}} & =\{x, y\}-\left\{x, \chi^{1}\right\} C_{12}\left\{\chi^{2}, y\right\}-\left\{x, \chi^{2}\right\} C_{21}\left\{\chi^{1}, y\right\} \\
& =-\frac{c}{e B}
\end{aligned}
$$

A extensão quântica deste sistema deve ser feita por meio de

$$
\{f, g\}_{\mathrm{D}}=\frac{1}{i \hbar}[\hat{f}, \hat{g}]
$$

e então o resultado (2.16) para a não comutatividade das coordenadas aparece naturalmente aqui pelo método de quantização de Dirac de sistemas sujeitos a vínculos. Para um campo magnético constante, podemos escrever então

$$
\left[x^{i}, x^{j}\right]=i \theta^{i j},
$$

onde $\theta^{i j}$ é um tensor anti-simétrico constante, que neste caso pode ser representado por $\theta^{i j}=-\frac{\hbar c}{e B} \epsilon^{i j}$, onde $i, j=1,2$ e $\epsilon^{i j}$ é o tensor unitário totalmente anti-simétrico.

\subsubsection{Partícula carregada em um campo magnético intenso não-uniforme}

Motivados pela análise anterior, nos surgiu a seguinte questão: se o campo magnético não for uniforme, qual a generalização da relação de comutação clássica (2.23)? E a extensão quântica (2.16)? Será que podemos escrevê-la na forma

$$
\left[x^{i}, x^{j}\right]=i \theta^{i j}(\vec{r})
$$

com $i, j=1,2,3$ e $\theta^{i j}$ uma função local das coordenadas? A resposta é sim. Fizemos um estudo da generalização do sistema anterior para o caso de uma partículas sujeita a um campo magnético não- 
uniforme $\vec{B}(\vec{r})$, e o resultado é que a comutação quântica entre as coordenadas é generalizada para a forma

$$
\left[x^{i}, x^{j}\right]=-i \hbar \frac{c}{e} \epsilon^{i j k} \frac{B_{k}(\vec{r})}{B^{2}(\vec{r})}, \quad(i, j, k=1,2,3) .
$$

Para mostrar como chegamos a este resultado, vamos apresentar brevemente aqui o que foi feito. Para mais detalhes sugerimos consultar a referência [72].

No caso tridimensional, as equações de movimento que seguem da lagrangeana (2.1) com $\vec{A}$ e $V$ gerais são

$$
m \ddot{\vec{r}}=\frac{e}{c} \dot{\vec{r}} \times \vec{B}(\vec{r})+\vec{f}(\vec{r})
$$

onde $\vec{r}=\left(x^{1}, x^{2}, x^{3}\right)$ e $\vec{f}=-\vec{\nabla} V$ é a força derivada do potencial externo. No limite $m \rightarrow 0$ as equações de movimento se reduzem a

$$
\epsilon_{k i j} \dot{x}^{i} B^{j}+\frac{c}{e} f_{k}=0
$$

de onde segue diretamente a relação

$$
B^{k} f_{k}=\vec{B} \cdot \vec{f}=0
$$

que assegura que a força líquida na direção de $\vec{B}$ se anula, o que representa uma condição necessária para obter equações de movimento consistentes no limite $m \rightarrow 0$. De fato, como a força de Lorentz é ortogonal ao campo magnético, esta condição nos permite dizer que a projeção de $m \ddot{\vec{r}}$ ao longo de $\vec{B}$ é nula. Então, no caso em que a velocidade é perpendicular ao campo magnético, podemos inverter a equação (2.29) e obtermos as equações de movimento,

$$
\dot{x}^{i}=\frac{c}{e} \epsilon_{i j k} \frac{f^{j} B^{k}}{B^{2}}
$$

Por outro lado, a hamiltoniana é dada simplesmente por $H_{0}=V(\vec{r})$, e as equações de Hamilton fornecem

$$
\dot{x}^{i}=\left\{x^{i}, H_{0}\right\}=-f_{j}\left\{x^{i}, x^{j}\right\}
$$

e a única forma de obter corretamente as equações de movimento (2.31) é impondo a comutação

$$
\left\{x^{i}, x^{j}\right\}=-\frac{c}{e} \epsilon_{i j k} \frac{B^{k}}{B^{2}}
$$

que leva diretamente à relação (2.27) depois da quantização.

Podemos obter o mesmo resultado pelo método de Dirac. A hamiltoniana para o sistema é da forma geral

$$
H=\frac{\vec{\pi}^{2}}{2 m}+V(\vec{r})=\frac{1}{2 m}\left(\vec{p}-\frac{e}{c} \vec{A}\right)^{2}+V(\vec{r})
$$


onde $\vec{\pi}$ é o momento cinético, $\vec{p}$ é o momento canônico e $\vec{B}=\vec{\nabla} \times \vec{A}$. Para que possamos tomar o limite $m \rightarrow 0$, devemos impor os vínculos $\vec{\pi} \approx 0$, que correspondem a

$$
\pi^{i}=p^{i}-\frac{e}{c} A^{i} \approx 0 \quad(i=1,2,3)
$$

e satisfazem

$$
\left\{\pi^{i}, \pi^{j}\right\}=\frac{e}{c}\left(\partial^{i} A^{j}-\partial^{j} A^{i}\right)=\frac{e}{c} \epsilon^{i j k} B_{k}
$$

É fácil verificar que com estes três vínculos podemos construir dois outros vínculos de segunda classe,

$$
\chi^{1}=\vec{f} \cdot \vec{\pi} ; \quad \chi^{2}=(\vec{B} \times \vec{f}) \cdot \vec{\pi} .
$$

São estes vínculos que devemos usar em (2.17) e (2.18) para obtermos o comutador de Dirac. Usando as relações (2.8) obtemos

$$
\left\{x^{i}, \chi^{1}\right\}=f^{i} ; \quad\left\{x^{i}, \chi^{2}\right\}=\epsilon^{i j k} B_{j} f_{k} ; \quad\left\{\chi^{1}, \chi^{2}\right\}=\frac{e}{c} B^{2} f^{2}
$$

de forma que chegamos finalmente a

$$
\left\{x^{i}, x^{j}\right\}_{D}=-\frac{c}{e} \epsilon^{i j k} \frac{B_{k}(\vec{r})}{B^{2}(\vec{r})}
$$

Portanto, a quantização leva diretamente à (2.27), mostrando que neste caso a não-comutatividade das coordenada é uma função local do espaço.

Como aplicação do resultado anterior, considere o caso de um campo magnético fracamente não uniforme na direção $z$. Um campo deste tipo ocorre em um espelho magnético, produzido por um par de espiras alinhadas, como em uma bobina de Helmholtz. Este sistema tem a propriedade de confinar o movimento de uma partícula dentro do espelho, onde o campo magnético pode ser descrito em coordenadas cilíndricas por

$$
\vec{B}=-\frac{1}{2} \rho \frac{\partial B_{z}(z)}{\partial z} \hat{\boldsymbol{e}}_{\boldsymbol{\rho}}+B_{z}(z) \hat{\boldsymbol{e}}_{\boldsymbol{z}}
$$

onde $\rho B_{z}^{\prime}<<B_{z}$. Então, a solução (2.27) implica nas seguintes relações de não-comutatividade entre as coordenadas

$$
[x, y]=-i \hbar \frac{c}{e} \frac{B_{z}}{B^{2}} \quad ; \quad[y, z]=i \hbar \frac{c}{2 e} \frac{x B_{z}^{\prime}}{B^{2}} \quad ; \quad[z, x]=i \hbar \frac{c}{2 e} \frac{y B_{z}^{\prime}}{B^{2}}
$$

Vemos que a intensidade da não-comutatividade é mais forte no plano $x-y$, enquanto que nos planos $x-z$ e $y-z$ ela é enfraquecida por um fator $\rho B_{z}^{\prime} / B_{z}<<1$. A quantização deste sistema no caso bidimensional com um campo magnético constante foi estudado por Landau [21], e os níveis de energia associados são os conhecidos níveis de Landau, infinitamente degenerados. Fizemos um estudo da quantização deste sistema 
quando o campo magnético é não uniforme, da forma (2.40), onde apresentamos uma generalização aos níveis de energia de Landau, que são obtidos no limite de campo constante. Estes resultados estão na referência [73].

Antes de começarmos a estudar a formulação de teorias de campos em espaços não-comutativos, devemos fazer alguns comentários. A não-comutatividade do espaço não é só uma abstração matemática no sentido de generalizar alguns resultados. Muitos trabalhos têm sido publicados ultimamente sugerindo testes concretos para a não-comutatividade ser de fato medida. Um trabalho de Chaichian e colaboradores [19] tenta explicar o efeito $\operatorname{PVLAS}^{6}$ observado recentemente, que é a rotação da polarização da luz propagando-se no vácuo na presença de um campo magnético de fundo. Outros testes à não-comutatividade foram propostos pelos mesmos autores anteriormente [29], por exemplo eles calcularam qual o efeito da não-comutatividade nos níveis de energia do átomo de hidrogênio e no Lamb shift, quais as correções ao efeito Stark e ao efeito Zeeman. Também vale citar trabalhos recentes que tentam incorporar a nãocomutatividade à relatividade geral e à gravitação [30, 31, 32].

\subsection{Aspectos gerais de teorias de campos não-comutativos}

Nesta seção vamos implementar as mudanças necessárias na teoria de campos usual para incluir efeitos da não-comutatividade.

O estudo de teorias de campos definidas em variedades onde as coordenas do espaço-tempo satisfazem à relação

$$
\left[x^{\mu}, x^{\nu}\right]=i \theta^{\mu \nu}
$$

tem aumentado nos últimos anos. Como já foi dito no início, a teoria de cordas é um cenário comum onde a não-comutatividade aparece naturalmente. Sendo um pouco mais específico, quando se estuda a teoria de cordas no regime de baixas energias na presença de um campo de fundo constante, a teoria resultante é bem descrita por uma teoria de campos definida no espaço não-comutativo ${ }^{7}$. Talvez esta seja a maior motivação para se estudar teorias não-comutativas, pois a teoria de cordas é esperada ser o ponto de partida no sentido de uma grande unificação. Vários outros autores descrevem com clareza e detalhes a formulação de teorias de campos em espaços não-comutativos. As referências [36, 37, 38, 39, 40] são especialmente completas e detalhadas no aspecto geral e com aplicações diversas. Vamos expor aqui apenas as propriedades e características principais que aparecem no tratamento de teorias de campos não-comutativos. Para maiores detalhes sugerimos as referências citadas acima.

A primeira regra para tratarmos teorias de campos em espaços não-comutativos é substituir o produto dos campos, que aparece na ação da teoria por exemplo, pelo chamado produto estrela de Grönewold-

\footnotetext{
${ }^{6}$ Polarizzazione del Vuoto con LASer (Polarização do vácuo com laser).

${ }^{7}$ Este resultado foi apresentado primeiramente por Seiber e Witten [35]. Uma discussão mais detalhada e mais pedagógica pode ser encontrada nas págs. 22-34 da dissertação de mestrado de Bruno Charneski, ref. [33].
} 
Moyal entre duas funções, definido por

$$
\begin{aligned}
f(x) \star g(x) & =\left.\exp \left(\frac{i}{2} \theta^{\mu \nu} \frac{\partial}{\partial x^{\mu}} \frac{\partial}{\partial y^{\nu}}\right) f(x) g(y)\right|_{x=y} \\
& =f(x) g(x)+\frac{i}{2} \theta^{\mu \nu} \partial_{\mu} f \partial_{\nu} g+\frac{1}{2 !}\left(\frac{i}{2}\right)^{2} \theta^{\mu \nu} \theta^{\alpha \beta}\left(\partial_{\mu} \partial_{\alpha} f\right)\left(\partial_{\nu} \partial_{\beta} g\right)+\cdots
\end{aligned}
$$

onde, na segunda linha, temos a expansão em termos do produto ordinário das funções $f$ e $g$ e suas derivadas em todas as ordens. Note que o primeiro termo é o produto ordinário das funções, que é o limite esperado quando $\theta \rightarrow 0$. Este produto introduz de maneira natural o chamado comutador Moyal ${ }^{8}$ de duas funções

$$
\begin{aligned}
{[f, g]_{\mathrm{MB}} } & =f(x) \star g(x)-g(x) \star f(x) \\
& =2 i f(x) \sin \left(\frac{1}{2} \overleftarrow{\partial_{\mu}} \theta^{\mu \nu} \overrightarrow{\partial_{\nu}}\right) g(x)
\end{aligned}
$$

onde a seta na primeira derivada parcial significa que ela atua na função $f$, à esquerda, e a segunda atua em $g$, à direita ${ }^{9}$. A forma dada na segunda linha obtém-se facilmente utilizando-se a expressão expandida em (2.43). As potências pares em $\theta^{\mu \nu}$ anulam-se, sobrando apenas as potências ímpares, que podem ser reagrupadas de forma a serem escritas como a função seno. Da mesma forma define-se o anticomutador Moyal

$$
\begin{aligned}
{[f, g]_{\mathrm{MB}}^{+} } & =f(x) \star g(x)+g(x) \star f(x) \\
& =2 f(x) \cos \left(\frac{1}{2} \overleftarrow{\partial_{\mu}} \theta^{\mu \nu} \overrightarrow{\partial_{\nu}}\right) g(x)
\end{aligned}
$$

Da definição (2.44) segue diretamente a comutação entre as coordenadas (2.42)

$$
\left[x^{\mu}, x^{\nu}\right]_{\mathrm{MB}}=i \theta^{\mu \nu},
$$

por isso podemos dizer que a álgebra definida por (2.42) implica que o produto entre funções deve ser tomado de acordo com (2.43).

Outra propriedade importante é a integração do produto de funções. Pode-se mostrar que a integral $d$-dimensional do produto Moyal de duas funções é igual à integral do produto ordinário das funções

$$
\int \mathrm{d}^{d} x f(x) \star g(x)=\int \mathrm{d}^{d} x f(x) g(x),
$$

\footnotetext{
${ }^{8} \mathrm{Na}$ literatura chama-se Moyal Bracket, por isso o subscrito MB.

${ }^{9}$ Esta é apenas uma notação alternativa à dada na eq. (2.43), onde está explícito que as derivadas agem em $x$ e em $y$ sem ambiguidade.
} 
e para o produto de $n$ funções vale a propriedade de ciclicidade

$$
\int \mathrm{d}^{d} x f_{1}(x) \star f_{2}(x) \star \cdots \star f_{n}(x)=\int \mathrm{d}^{d} x f_{n}(x) \star f_{1}(x) \star \cdots \star f_{n-1}(x) .
$$

Outras duas propriedades importantes são ${ }^{10}$ :

$$
\begin{gathered}
\mathrm{e}^{i k x} \star \mathrm{e}^{i k^{\prime} x}=\mathrm{e}^{-\frac{i}{2} \theta^{i j} k_{i} k_{j}^{\prime}} \mathrm{e}^{i\left(k+k^{\prime}\right) x} \\
\mathrm{e}^{i k x} \star f(x) \star \mathrm{e}^{-i k x}=\mathrm{e}^{\theta^{i j} k_{i} \partial_{j}} f(x)=f\left(x^{i}+\theta^{i j} k_{j}\right),
\end{gathered}
$$

que podem ser obtidas por expansão do produto Moyal e reagrupamento dos termos de forma conveniente.

No que segue vamos estudar a eletrodinâmica quântica definida em um espaço não-comutativo ${ }^{11}$, usando as propriedades anteriores.

\subsection{QED não-comutativa}

Nesta seção vamos apresentar os principais resultados já existentes na literatura de estudos da eletrodinâmica quântica não-comutativa. Vamos apresentar as regras de Feynman da teoria e os diagramas em 1-loop que contribuem por exemplo para a auto-energia do fóton. O objetivo é introduzir a NCQED para que possamos fazer cálculos a temperatura finita no próximo capítulo.

\subsection{Ação da QED não-comutativa}

A formulação da eletrodinâmica quântica em espaços não-comutativos difere um pouco da QED usual (comutativa). Como vimos, a não-comutatividade das coordenadas do espaço-tempo definida por (2.42) implica numa redefinição do produto de funções, que deve obedecer ao produto de Grönewold-Moyal (2.43). A eletrodinâmica quântica também pode ser formulada em um espaço não-comutativo desse tipo, precisamos apenas tomar alguns cuidados. Em primeiro lugar, para evitar problemas com a unitariedade da teoria, vamos assumir que somente as componentes espaciais de $\theta^{\mu \nu}$ são diferentes de zero, ou seja, $\theta^{00}=\theta^{i 0}=\theta^{0 i}=0$. Em segundo lugar, o tensor eletromagnético $F^{\mu \nu}$ deve ser generalizado. Para que a lagrangeana seja invariante por transformações de gauge, devemos ter

$$
F_{\mu \nu}=\partial_{\mu} A_{\nu}-\partial_{\nu} A_{\mu}-i e\left[A_{\mu}, A_{\nu}\right]_{\mathrm{MB}}
$$

e a derivada covariante agindo em um campo de gauge $A^{\nu}$ deve ser da forma

$$
D_{\mu} A^{\nu}=\partial_{\mu} A^{\nu}-i e\left[A_{\mu}, A^{\nu}\right]_{\mathrm{MB}}
$$

\footnotetext{
${ }^{10}$ Ver pág. 5 de [36].

${ }^{11}$ Neste capítulo e nos que seguem, na maioria das vezes vamos nos referir à eletrodinâmica quântica não-comutativa simplesmente como NCQED (Noncommutative Quantum Electrodynamics), como encontramos usualmente na literatura. A eletrodinâmica quântica usual (comutativa) será denominada simplesmente por QED ou QED usual.
} 
e quando agindo em um campo fermiônico $\psi$

$$
D_{\mu} \psi=\partial_{\mu} \psi-i e A_{\mu} \star \psi
$$

Com isto, a ação para a QED não-comutativa deve ser definida como ${ }^{12}$

$$
S_{\mathrm{inv}}=\int \mathrm{d}^{4} x \mathcal{L}_{\mathrm{inv}}=\int \mathrm{d}^{4} x\left(-\frac{1}{4} F_{\mu \nu} \star F^{\mu \nu}+\bar{\psi} \star(i \not D-m) \psi\right) .
$$

Esta ação é invariante por uma transformação de gauge do tipo

$$
\begin{aligned}
\psi(x) & \rightarrow \psi^{\prime}(x)=U(x) \star \psi(x) \\
A_{\mu}(x) & \rightarrow A_{\mu}^{\prime}(x)=U(x) \star A_{\mu}(x) \star U^{-1}(x)+\frac{i}{e} U(x) \star \partial_{\mu} U^{-1}(x) .
\end{aligned}
$$

Do ponto de vista infinitesimal, esta transformação toma a seguinte forma

$$
\begin{aligned}
\delta \psi(x) & =i \epsilon(x) \star \psi(x) \\
\delta A_{\mu} & =\frac{1}{e} D_{\mu} \epsilon(x)=\frac{1}{e}\left(\partial_{\mu} \epsilon-i e\left[A_{\mu}, \epsilon\right]_{\mathrm{MB}}\right)
\end{aligned}
$$

onde $\epsilon(x)$ é o parâmetro da transformação infinitesimal. A esta ação podemos ainda somar um termo fixador de gauge (fg) e um termo devido a campos fantasmas (gh) ${ }^{13}$. Estes termos devem contribuir com

$$
S_{\mathrm{fg}}+S_{\mathrm{gh}}=\int \mathrm{d}^{4} x\left(-\frac{1}{2 \xi}\left(\partial_{\mu} A^{\mu}\right) \star\left(\partial_{\nu} A^{\nu}\right)+\partial^{\mu} \bar{C} \star\left(\partial_{\mu} C-i e\left[A_{\mu}, C\right]_{\mathrm{MB}}\right)\right)
$$

onde $\xi$ é parâmetro fixador de gauge. Desta forma, a ação completa da QED não-comutativa é expressa por:

$$
\begin{aligned}
& S_{N C Q E D}=S_{\mathrm{inv}}+S_{\mathrm{fg}}+S_{\mathrm{gh}} \\
&=\int \mathrm{d}^{4} x\left(-\frac{1}{4} F_{\mu \nu} \star F^{\mu \nu}+\bar{\psi} \star(i \not D-m) \psi-\frac{1}{2 \xi}\left(\partial_{\mu} A^{\mu}\right) \star\left(\partial_{\nu} A^{\nu}\right)\right. \\
&\left.+\partial^{\mu} \bar{C} \star\left(\partial_{\mu} C-i e\left[A_{\mu}, C\right]_{\mathrm{MB}}\right)\right) .
\end{aligned}
$$

Fica fácil perceber desta forma da ação que ela tem a mesma estrutura de uma teoria de gauge nãoabeliana $^{14}$, sendo o produto Moyal o responsável por esta estrutura não-abeliana, explícito no último termo do tensor eletromagnético (2.51). Aliás, são muitas as analogias entre uma teoria de gauge $S U(N)$

\footnotetext{
${ }^{12}$ Em teorias não-comutativas é mais comum escrever a ação da teoria, ao invés da lagrangeana, pois em geral a invariância de gauge é satisfeita pela ação neste caso, e não pela lagrangeana propriamente dita, devido à necessidade do uso de integrações por partes para se justificar a invariância.

${ }^{13}$ Lembre-se que este termo é necessário mesmo na QED usual para incluir corretamente o número de graus de liberdade em cálculos a temperatura finita, conforme discutido na seção 1.2.3 para o caso do gás de fótons.

${ }^{14}$ Teoria de Yang-Mills por exemplo.
} 
não-abeliana definida no espaço comutativo usual e uma teoria de gauge $U(N)$ definida no espaço nãocomutativo.

Vamos ver agora como ficam as regras de Feynman para esta teoria.

\subsubsection{Regras de Feynman da NCQED}

O produto Moyal tem conseqüências interessantes nas regras de Feynman da teoria. Como vimos em (2.47), quando o produto Moyal de duas funções é integrado, o resultado é o mesmo que no produto ordinário das funções ${ }^{15}$. Isto se reflete no fato de que os propagadores da teoria não mudam, são os mesmos da teoria comutativa. Portanto, para os propagadores temos as seguintes regras:

férmion

$$
\stackrel{p}{\longrightarrow}: \quad \frac{i}{p-m+i \epsilon}=i S(p)
$$

fóton

$$
\mu \sim \sim^{v} \sim \sim^{v}: \quad-\frac{i}{\left(p^{2}+i \epsilon\right)}\left(\eta_{\mu \nu}-(1-\xi) \frac{p_{\mu} p_{\nu}}{p^{2}}\right)=i D_{\mu \nu}(p)
$$

ghost

$$
--\underline{p}---: \quad \frac{i}{p^{2}+i \epsilon}=i D(p)
$$

que são as mesmas de (1.2) e (1.3) da QED usual, com a adição do propagador $D(p)$ devido aos campos fantasmas (ghosts).

Outra característica é que, sob uma integração, qualquer número de funções multiplicadas segundo o produto Moyal satisfazem a propriedade de ciclicidade, conforme (2.48). Isto leva a uma modificação dos vértices da teoria. Eles devem depender do parâmetro de não-comutatividade $\theta^{\mu \nu}$. De fato isto acontece. Para entendermos como ficam os vértices da teoria, vamos detalhar um pouco como aparece um deles. Da análise direta da ação (2.58), vemos que aparecem vértices contendo três e quatro linhas de fótons, assim como vértices contendo dois campos fermiônicos e um campo de fóton, e o mesmo para campos fantasmas. Por exemplo, o vértice com três linhas de fótons é originado de um termo do tipo

$$
\left(\partial_{\mu} A_{\nu}(x)-\partial_{\nu} A_{\mu}(x)\right) \star(-i e)\left[A^{\mu}(x), A^{\nu}(x)\right]_{\mathrm{MB}}
$$

vindo do primeiro termo da ação. Vamos desenvolver o primeiro termo desta expressão. Ele pode ser

\footnotetext{
${ }^{15}$ Basta fazer uma integração por partes na expressão expandida do produto e considerar que as funções se anulam no infinito.
} 
escrito como

$$
\begin{aligned}
\partial_{\mu} A_{\nu}(x) \star & (-i e)\left[A^{\mu}(x), A^{\nu}(x)\right]_{\mathrm{MB}} \\
& =-i e \partial_{\mu} A_{\nu}(x) \star\left[A^{\mu}(x), A^{\nu}(x)\right]_{\mathrm{MB}} \\
& =-i e \partial_{\mu} A_{\nu}(x) \star \eta^{\alpha \mu}\left[A_{\alpha}(x), A_{\beta}(x)\right]_{\mathrm{MB}} \eta^{\beta \nu} \\
& =-i e \partial_{\mu} A_{\nu}(x) \star \eta^{\alpha \mu}\left(A_{\alpha}(x) \star A_{\beta}(x)-A_{\beta}(x) \star A_{\alpha}(x)\right) \eta^{\beta \nu} \\
& =-i e \eta^{\alpha \mu} \partial_{\mu} A_{\nu}(x) \star\left(2 i A_{\alpha}(x) \sin \left(\frac{1}{2} \overleftarrow{\partial_{j}} \theta^{j k} \overrightarrow{\partial_{k}}\right) A_{\beta}(x)\right) \eta^{\beta \nu}
\end{aligned}
$$

onde usamos a propriedade (2.44) na última linha. Agora, para que as derivadas possam agir nos campos de gauge $A(x)$, escrevemos para cada um deles uma expansão de Fourier do tipo

$$
A_{\alpha}(x)=\int \mathrm{d}^{4} p_{1} A_{\alpha}\left(p_{1}\right) \mathrm{e}^{i p_{1} \cdot x}
$$

e similarmente para $A_{\beta}(x)$ e $A_{\nu}(x)$, com integração nos momentos $p_{2}$ e $p_{3}$, respectivamente. Desta forma, o efeito das derivadas é gerar um termo do tipo $\left(i p_{1}\right)$ por exemplo, cada um com o momento correspondente. Fazendo isto na última linha de (2.60) obtemos

$$
\begin{array}{r}
-i e(2 i) \eta^{\alpha \mu}\left(i p_{3 \mu}\right) A_{\nu}(x) \star\left[A_{\alpha}(x) \sin \left(\frac{1}{2}\left(i p_{1 j}\right) \theta^{j k}\left(i p_{2} k\right)\right) A_{\beta}(x)\right] \eta^{\beta \nu} \\
=-2 i e p_{3}^{\alpha} \sin \left(\frac{p_{1} \times p_{2}}{2}\right) \eta^{\beta \nu} A_{\nu}(x) \star A_{\alpha}(x) A_{\beta}(x),
\end{array}
$$

onde introduzimos a notação $p \times q=p_{\mu} \theta^{\mu \nu} q_{\nu}$, que será muito utilizada daqui em diante. $\mathrm{O}$ único produto Moyal que resta é idêntico ao produto ordinário das funções, como já vimos, pois esta expressão está sob uma integração. A conservação do momento requer que $p_{3}=p_{1}-p_{2}$. Desta forma, retirando os campos $A$ 's da expressão, o que resta é um termo do tipo ${ }^{16}$

$$
-2 e \sin \left(\frac{p_{1} \times p_{2}}{2}\right)\left(p_{1}-p_{2}\right)^{\alpha} \eta^{\beta \nu},
$$

que deve ser um termo típico do fator de vértice para o caso de três linhas de fótons. De fato é exatamente isso que acontece. Os fatores de vértice são dados por

\footnotetext{
${ }^{16} \mathrm{O}$ fator $i$ é absorvido por $\exp i S$.
} 

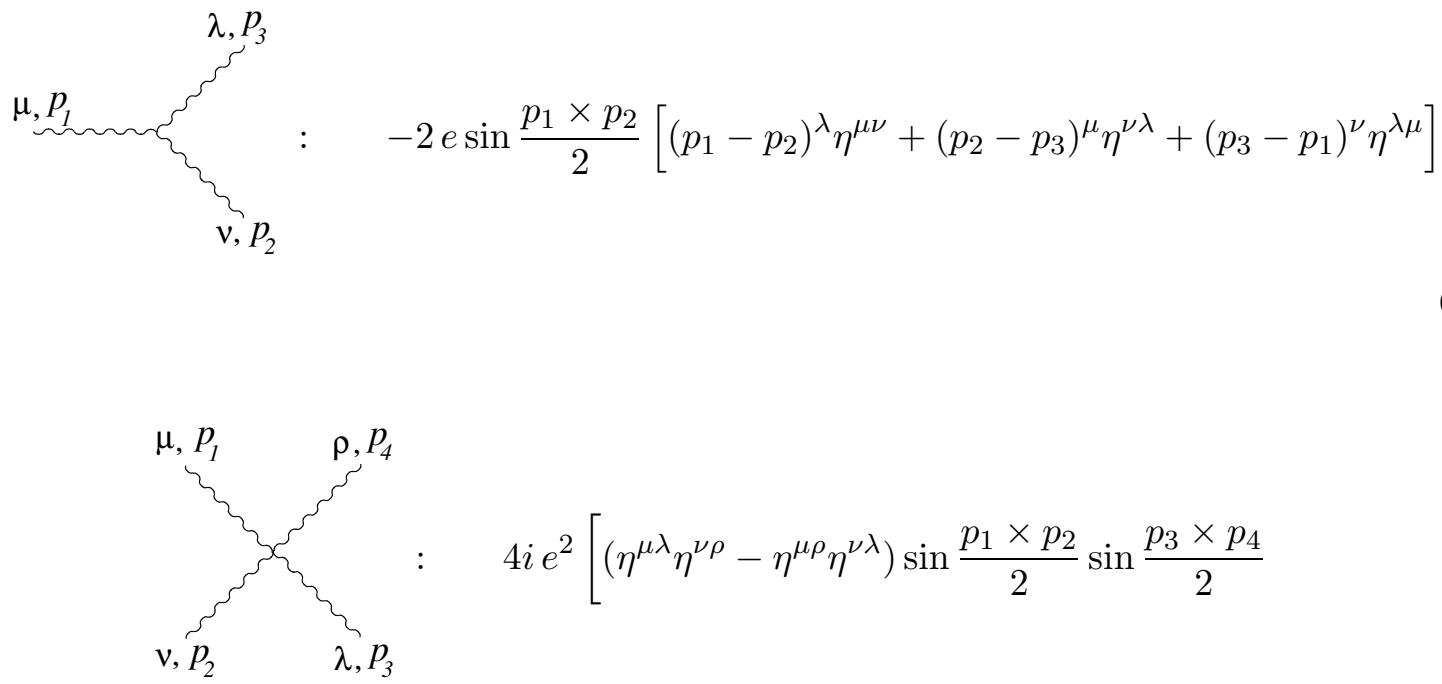

$$
\begin{aligned}
4 i e^{2}\left[\left(\eta^{\mu \lambda} \eta^{\nu \rho}-\eta^{\mu \rho} \eta^{\nu \lambda}\right) \sin \frac{p_{1} \times p_{2}}{2} \sin \frac{p_{3} \times p_{4}}{2}\right. \\
\quad+\left(\eta^{\mu \rho} \eta^{\nu \lambda}-\eta^{\mu \nu} \eta^{\lambda \rho}\right) \sin \frac{p_{3} \times p_{1}}{2} \sin \frac{p_{2} \times p_{4}}{2}
\end{aligned}
$$

$$
\left.+\left(\eta^{\mu \nu} \eta^{\lambda \rho}-\eta^{\mu \lambda} \eta^{\nu \rho}\right) \sin \frac{p_{1} \times p_{4}}{2} \sin \frac{p_{2} \times p_{3}}{2}\right]
$$
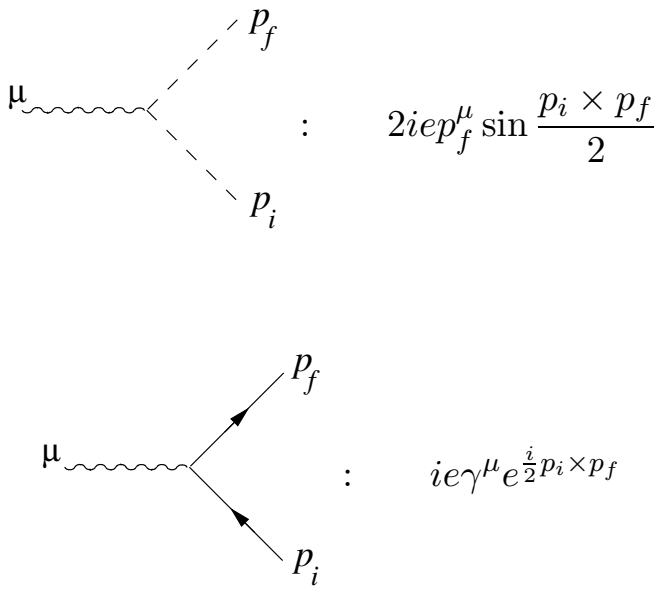

A dedução de cada um deles segue de maneira similar à análise que fizemos para o caso do vértice com três fótons.

\subsubsection{Auto-energia do fóton}

Vamos estudar aqui a a auto-energia do fóton, cujas contribuições vêm dos gráficos de 1-loop representados na Figura 2.1. 


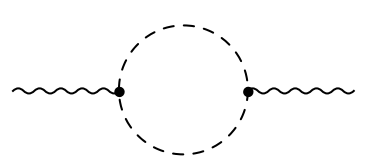

(a)

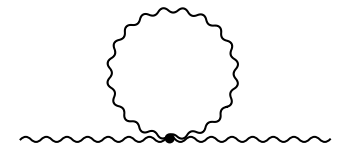

(b)

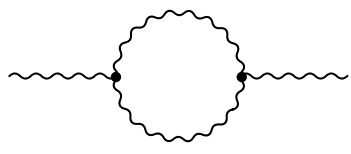

(c)

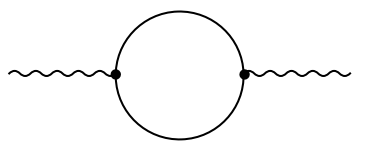

$(d)$

Figura 2.1: Contribuições para auto-energia do fóton na eletrodinâmica quântica não-comutativa.

Vamos então obter as expressões correspondentes para cada um dos diagramas que contribuem para o tensor de auto-energia do fóton $\Pi^{\mu \nu}(p)$. Uma das contribuições vem do gráfico de 1-loop de ghost da Figura 2.1(a), que pode ser representado com detalhes pela Figura 2.2. Neste capítulo e nos seguintes vamos usar sempre $k$ para indicar o momento interno ao loop e $p$ para indicar o momento externo, ao contrário do que foi usado no capítulo anterior. Usando as regras de Feynman dadas em (2.59) e (2.64)

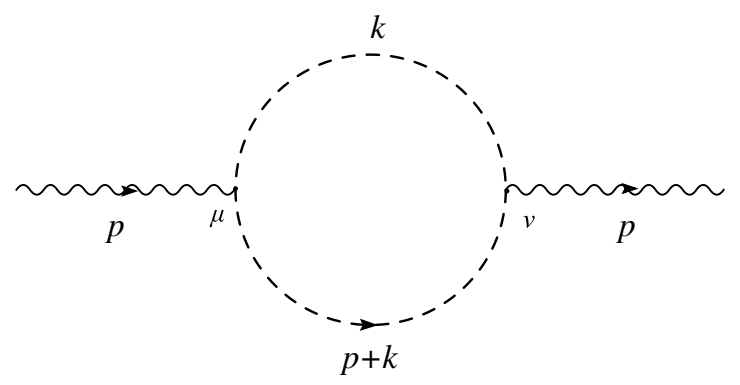

Figura 2.2: Diagrama de 1-loop de ghost que contribui para a auto-energia do fóton na NCQED.

podemos escrever a contribuição deste gráfico como

$$
\Pi_{a}^{\mu \nu}(p)=(-1)\left(-4 e^{2}\right) \int \frac{\mathrm{d}^{4} k}{(2 \pi)^{4}} \sin ^{2}\left(\frac{k \times p}{2}\right) \frac{k^{\nu}\left(k^{\mu}+p^{\mu}\right)}{k^{2}(k+p)^{2}} .
$$

O fator $(-1)$ vem do loop de $g h o s t^{17}$.

O diagrama da Figura 2.1(b), conhecido como tadpole, pode ser representado em detalhes conforme a Figura 2.3. Usando as regras de Feynman (2.59) e (2.63) e restringindo ao gauge de Feynman, onde $\xi=1$,

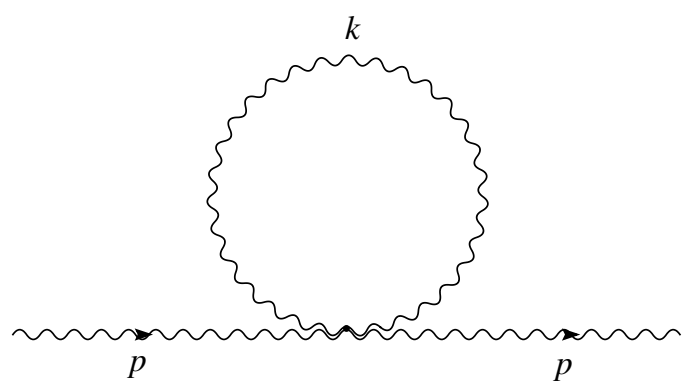

Figura 2.3: Diagrama do tipo tadpole que contribui para auto-energia do fóton na NCQED.

\footnotetext{
${ }^{17}$ Campos de ghost têm a mesma propriedade que campos fermiônicos, por isso vale a regra 7 dada na seção 1.1.1.
} 
podemos escrever a contribuição deste gráfico como

$$
\Pi_{b}^{\mu \nu}(p)=\frac{1}{2} 24 e^{2} \int \frac{\mathrm{d}^{4} k}{(2 \pi)^{4}} \sin ^{2}\left(\frac{k \times p}{2}\right) \frac{\eta^{\mu \nu}}{k^{2}}
$$

onde o fator (1/2) é o fator combinatorial associado a este tipo de diagrama.

Para o gráfico da Figura 2.1(c), a representação detalhada está na Figura 2.4. Usando as regras de

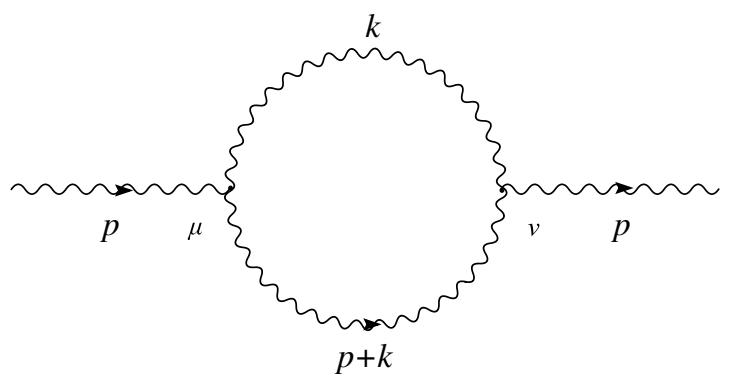

Figura 2.4: Diagrama de 1-loop de fóton que contribui para a auto-energia do fóton na NCQED.

Feynman a contribuição deste gráfico é

$$
\begin{aligned}
& \Pi_{c}^{\mu \nu}(p)= \\
& -\frac{1}{2} 4 e^{2} \int \frac{\mathrm{d}^{4} k}{(2 \pi)^{4}} \sin ^{2}\left(\frac{k \times p}{2}\right)\left[\frac{\left(5 p^{2}+2 k \cdot p+2 k^{2}\right) \eta^{\mu \nu}-2 p^{\mu} p^{\nu}+5\left(k^{\mu} p^{\nu}+p^{\mu} k^{\nu}\right)+10 k^{\mu} k^{\nu}}{k^{2}(k+p)^{2}}\right]
\end{aligned}
$$

lembrando que estamos nos restringindo ao gauge de Feynman. O fator (1/2) também se deve ao fator combinatorial do diagrama.

Por último, considere o diagrama do loop fermiônico, representado na Figura 2.5. A expressão para

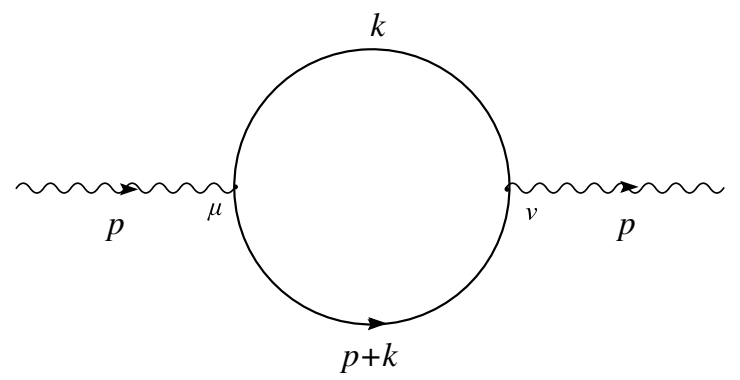

Figura 2.5: Diagrama de 1-loop fermiônico que contribui para a auto-energia do fóton na NCQED.

este diagrama é dada por

$$
\Pi_{d}^{\mu \nu}(p)=-e^{2} \int \frac{\mathrm{d}^{4} k}{(2 \pi)^{4}} \operatorname{tr}\left(\gamma^{\mu} \frac{1}{\not k+\not p-m} \gamma^{\nu} \frac{1}{\not k-m}\right) .
$$


A contribuição total para a auto-energia do fóton na eletrodinâmica quântica não-comutativa será dada pela soma

$$
\Pi^{\mu \nu}(p)=\Pi_{a}^{\mu \nu}(p)+\Pi_{b}^{\mu \nu}(p)+\Pi_{c}^{\mu \nu}(p)+\Pi_{d}^{\mu \nu}(p)
$$

No entanto, vejam que a expressão do último diagrama, o de loop fermiônico, não tem dependência com o parâmetro de não-comutatividade $\theta^{i j}$, sendo que todos os outros têm uma dependência no fator trigonométrico, do tipo $\sin ^{2}\left(k_{i} \theta^{i j} p_{j} / 2\right)$. Isto se deve ao fato de que o fator de vértice (2.65) é do tipo exponencial, e portanto há um cancelamento do expoente. De fato, a expressão obtida para este diagrama é exatamente idêntica à obtida em (1.14), basta tomar $c=1$ naquele caso. Concluímos com isso que a parte fermiônica da eletrodinâmica quântica não-comutativa em 1-loop é exatamente igual à da teoria comutativa. Para estudarmos contribuições vindas da parte não-comutativa basta nos restringirmos aos três primeiros diagramas somente.

Como dissemos antes, as expressões que compõem o tensor de auto-energia do fóton (2.70) foram obtidas no gauge de Feynman, o que simplifica muito a expressão final. Um tratamento mais detalhado em uma dimensão arbitrária do espaço-tempo e em um gauge covariante geral foi estudado por Frenkel, Das e Brandt [41], onde eles calcularam a integração em $k$ explicitamente. Como resultado eles encontraram que o tensor de auto-energia é transversal em todas as ordens para um gauge geral em $d$ dimensões. Os cálculos foram feitos explicitamente em 1-loop, e para isto uma generalização das fórmulas de regulariação dimensional foram desenvolvidas para incorporar os efeitos de não-comutatividade. Eles também obtiveram que a auto-energia é dependente de gauge, da mesma forma que a QCD usual a temperatura nula ${ }^{18}$, mas não se desenvolve nenhum tipo novo de divergência ultravioleta, de forma que a teoria é renormalizável. Aqui estamos interessados apenas na forma do tensor, pois nosso objetivo é calculá-lo levando em conta a temperatura finita do sistema, o que faremos no capítulo seguinte.

Analisando um pouco mais a fundo as expressões dos diagramas que dependem do parâmetro de nãocomutatividade, percebemos algumas características interessantes. Primeiro note que todos eles dependem de $\theta$ da mesma forma, através do fator trigonométrico

$$
\sin ^{2}\left(\frac{k \times p}{2}\right)=\frac{1}{2}(1-\cos k \times p)
$$

que segue diretamente da identidade trigonométrica (C.13). Desta forma vemos que esses diagramas são formados por duas partes distintas, uma totalmente independente do parâmetro $\theta$ e outra dependente. A parte independente é chamada usualmente na literatura de parte "planar", enquanto que a parte dependente da não-comutatividade é chamada parte "não-planar". Observe também que se $\theta=0$ estas contribuições se anulam, como deveria, afinal isto significa que nenhum dos vértices (2.62)-(2.64) existe, portanto não há auto-interação entre os fótons. Observe também que todos os integrandos, exceto pelo fator trigonométrico, são proporcionais ${ }^{19}$ a $k$, portanto a integral é quadraticamente divergente, quando $k \rightarrow \infty$, para a parte

\footnotetext{
${ }^{18}$ A QED usual a temperatura nula é independente de gauge em 1-loop.

${ }^{19}$ Lembre-se que o elemento de volume em quatro dimensões contribui com $k^{3}$.
} 
planar. Isto é conhecido como "divergência ultravioleta", e a renormalização usual da QED se aplica da mesma forma aqui. No entanto a parte não-planar é convergente para $k \rightarrow \infty$, pois a parte trigonométrica oscila de forma a melhorar a convergência no ultravioleta. No entanto, como acontece em geral em teorias não-comutativas, o cálculo explícito da integral da parte não-planar mostra uma dependência com $\theta \mathrm{e}$ com o momento externo $p$ do tipo ${ }^{20} 1 /(\theta p)^{2}$, de forma que aparece agora uma divergência no setor de baixos momentos externos, $p \rightarrow 0$. Isto é conhecido como "divergência infravermelha". Portanto há uma "mistura" das divergências ultravioletas (UV) e infravermelhas (IR) quando a não-comutatividade está presente, e na literatura isto é conhecido como "UV/IR mixing". Uma boa discussão neste assunto foi feita por Szabo [37] e por Girotti [38]. Aplicações diversas de teorias não-comutativas foram feitas, citamos, por exemplo, estudos de teorias de Chern-Simons supersimétricas [42], modelos de Gross-Neveu tridimensional [43], estudos de renormalização da teoria [39, 44], estudos de invariância de gauge quando $\theta$ depende das coordenadas [45], entre outros.

\footnotetext{
${ }^{20}$ Veremos uma dependência deste tipo nos cálculos do próximo capítulo.
} 



\section{Capítulo 3}

\section{QED Não-Comutativa a Temperatura Finita}

Neste capítulo vamos apresentar alguns dos principais resultados de nosso trabalho. Vamos juntar a não-comutatividade do capítulo anterior com a eletrodinâmica quântica a temperatura finita do primeiro capítulo a fim de estudarmos qual a contribuição da não-comutatividade naqueles resultados. Começamos fazendo uma exposição mais detalhada do cálculo da auto-energia do fóton, mas devido a dificuldades em se calcular o tensor exatamente, cálculos exatos só podem serem feitos quando tomamos algum limite específico, e investigaremos o caso do chamado limite estático a altas temperaturas. As mesmas dificuldades já apareceram no capítulo 1, e aqui, com a presença do fator de não-comutatividade, os cálculos são ainda mais difíceis. Em seguida estudamos as blindagens dos campos elétrico e magnético, análogo ao que aparece na teoria usual a temperatura finita, descrito na seção 1.2.5. Depois calculamos as amplitudes de 3 e 4-pontos em 1-loop também no limite estático, e por meio de identidades de Ward conseguimos construir uma ação efetiva para a teoria em 1-loop. Finalizamos apresentando alguns resultados já presentes na literatura para a energia livre de Helmholtz e a pressão de um gás de fótons não-comutativos a altas temperaturas. Parte do material relacionado aos cálculos matemáticos foi deixado para o Apêndice. Com isto queremos evitar a perda de continuidade na exposição do tema principal. Todos os resultados apresentados neste capítulo, exceto os da seção 3.9, foram publicados, conforme ref. [70].

\subsection{Decomposição do tensor de auto-energia}

Como já foi dito no capítulo anterior, a contribuição ao propagador do fóton devido ao loop fermiônico não tem dependência com o parâmetro de não-comutatividade. Portanto a contribuição para a auto-energia com dependência em $\theta$ virá somente da parte dos campos de gauge da lagrangeana, ou seja, da parte envolvendo os campos do fóton.

Antes de iniciarmos o cálculo da função de 2-pontos, vamos discutir uma maneira conveniente de se decompor o tensor $\Pi^{\mu \nu}$, levando em conta tanto a temperatura finita quanto a não-comutatividade.

No caso comutativo a temperatura finita descrito na seção 1.2.4, vimos que, na sua forma mais ge- 
ral, o tensor de auto-energia devia depender de $\eta^{\mu \nu}$, do momento externo $p^{\mu}$ e da quadrivelocidade do reservatório térmico $u^{\mu}$. Em uma teoria não-comutativa temos mais um tensor do qual deve depender a auto-energia, que é o tensor $\theta^{\mu \nu}$, que caracteriza a não-comutatividade espacial ${ }^{1}$. Ele entra acoplado ao momento externo $p$ através da definição

$$
\tilde{p}^{\mu}=\theta^{\mu \nu} p_{\nu}
$$

Devido às identidades de Ward sabemos que o tensor de auto-energia deve ser transversal ao momento externo,

$$
p_{\mu} \Pi^{\mu \nu}=0
$$

Por definição $\tilde{p}^{\mu}$ é transversal a $p^{\mu}$, e podemos verificar que no caso $u^{\mu}=(1,0,0,0)$ temos $u \cdot \tilde{p}=0$, já que $\theta^{\mu \nu}$ envolve apenas os índices espaciais. Também temos $u \cdot p=0$, pois no limite estático $p_{0}=0$.

A forma geral do tensor $\Pi^{\mu \nu}$ deve ser

$$
\Pi^{\mu \nu}=P^{\mu \nu} \Pi_{\mathrm{T}}+Q^{\mu \nu} \Pi_{\mathrm{L}}+R^{\mu \nu} \tilde{\Pi}_{\mathrm{T}}
$$

com

$$
\begin{aligned}
P^{\mu \nu} & =\left(\eta^{\mu \nu}-u^{\mu} u^{\nu}-\frac{p^{\mu} p^{\nu}}{p^{2}}-\frac{\tilde{p}^{\mu} \tilde{p}^{\nu}}{\tilde{p}^{2}}\right), \\
Q^{\mu \nu} & =u^{\mu} u^{\nu} \\
R^{\mu \nu} & =\frac{\tilde{p}^{\mu} \tilde{p}^{\nu}}{\tilde{p}^{2}}
\end{aligned}
$$

Estas estruturas correspondem a operadores de projeção ortogonais e normalizados, como pode ser facilmente verificado,

$$
\begin{aligned}
& P^{\mu \nu} Q_{\mu \nu}=0, \quad Q^{\mu \nu} R_{\mu \nu}=0, \quad P^{\mu \nu} R_{\mu \nu}=0 \\
& P_{\mu}^{\mu}=1, \quad R_{\mu}^{\mu}=1, \quad Q_{\mu}^{\mu}=1 .
\end{aligned}
$$

Os coeficientes $\Pi_{\mathrm{T}}, \Pi_{\mathrm{L}}$ e $\tilde{\Pi}_{\mathrm{T}}$ são fatores de forma que devem ser determinados. Os subscritos "T" e "L" se referem, respectivamente, a 'transversal' e 'longitudinal', e a razão desta nomenclatura ficará mais claro adiante. A vantagem de se escrever o tensor na forma (3.3) é que estes fatores de forma podem ser determinados simplesmente fazendo-se a contração de $\Pi^{\mu \nu}$ com as três estruturas (3.4), de forma que

\footnotetext{
${ }^{1}$ Conforme discutido no capítulo anterior, vamos tomar $\theta^{00}=\theta^{0 i}=\theta^{i 0}=0$ para evitarmos problemas de unitariedade.
} 
obtém-se

$$
\begin{aligned}
& \Pi_{\mathrm{L}}=u_{\mu} u_{\nu} \Pi^{\mu \nu} \\
& \tilde{\Pi}_{\mathrm{T}}=\frac{\tilde{p}_{\mu} \tilde{p}_{\nu}}{\tilde{p}^{2}} \Pi^{\mu \nu} \\
& (d-3) \Pi_{\mathrm{T}}=\left(\eta_{\mu \nu}-\frac{p_{\mu} p_{\nu}}{p^{2}}\right) \Pi^{\mu \nu}-\Pi_{\mathrm{L}}-\tilde{\Pi}_{\mathrm{T}}
\end{aligned}
$$

Aqui $d$ representa a dimensão do espaço-tempo, e vem do termo $\eta^{\mu \nu} \eta_{\mu \nu}=d$. Note que para $d=3$ não temos nenhuma informação sobre o fator de forma transversal $\Pi_{\mathrm{T}}$, que para uma teoria convencional acontece quando $d=2$, conforme eq. (1.102).

O inverso do propagador exato pode ser escrito como

$$
\left(D^{-1}\right)^{\mu \nu}=P^{\mu \nu}\left(p^{2}-\Pi_{\mathrm{T}}\right)+Q^{\mu \nu}\left(p^{2}-\Pi_{\mathrm{L}}\right)+R^{\mu \nu}\left(p^{2}-\tilde{\Pi}_{\mathrm{T}}\right)+\frac{p_{\mu} p_{\nu}}{\xi}
$$

onde $\xi$ representa um parâmetro fixador de gauge em um gauge covariante. Como os operadores de projeção são ortonormais, o inverso da expressão acima pode ser facilmente calculado, levando ao propagador

$$
D_{\mu \nu}=P_{\mu \nu} \frac{1}{p^{2}-\Pi_{\mathrm{T}}}+Q_{\mu \nu} \frac{1}{p^{2}-\Pi_{\mathrm{L}}}+R_{\mu \nu} \frac{1}{p^{2}-\tilde{\Pi}_{\mathrm{T}}}+\xi \frac{p_{\mu} p_{\nu}}{p^{2}} .
$$

Os pólos que aparecem no propagador são distintos em conseqüência da nossa escolha dos operadores de projeção. Se tivéssemos usado uma base diferente, os pólos estariam misturados. Percebemos a presença de três pólos físicos e um vindo da fixação de gauge. Assim como no caso comutativo, estes pólos estão relacionados à blindagem dos campos elétrico e magnético, e serão discutidos mais à frente.

Vamos partir agora para o cálculo explícito do tensor de auto-energia do fóton a altas temperaturas.

\subsection{Auto-energia do fóton (amplitude de 2-pontos)}

Vamos então calcular a auto-energia do fóton $\Pi^{\mu \nu}(p)$ a temperatura finita. Como sabemos, os diagramas que contribuem para a auto-energia são dados na Figura 2.1 e a expressão correspondente para o tensor no gauge de Feynman a temperatura nula é dada por (2.70), excluindo-se o último termo, que corresponde ao loop fermiônico. Vamos trabalhar aqui no gauge de Feynman, $\xi=1$, pois conforme demonstrado por Frenkel e colaboradores em [46], em ordem dominante a auto-energia é independente de gauge a altas temperaturas.

A temperatura finita, o primeiro passo é calcular a soma sobre as freqüências $\omega_{n}$, que aparecem com a substituição (1.52), e existem vários métodos para isso. Vamos descrever três deles.

O primeiro método é fazer o cálculo diretamente das expressões de cada diagrama, por meio da soma sobre as frequiências, no entanto o cálculo exato não é muito simples de ser feito ${ }^{2}$, e ainda vai restar a

\footnotetext{
${ }^{2}$ Lembre-se do caso do loop fermiônico do capítulo 1 . A integração no momento interno não foi feita naquele caso, e só pode
} 
integração no momento interno, que só pode ser feita em algum limite específico, e o caso que nos interessa é o limite estático $\left(p_{0}=0, \vec{p} \rightarrow 0\right)$. Portanto, para fazermos a soma diretamente, é mais conveniente primeiro tomar o limite estático.

O segundo método, chamado método da amplitude de espalhamento frontal ${ }^{3}$, foi desenvolvido inicialmente por G. Barton e generalizado por Frenkel, Taylor e Brandt [47, 48] para uma classe geral de gauges covariantes. Este método nos diz basicamente que os gráficos que representam uma determinada amplitude podem ser escritos como a soma de gráficos que representam a amplitude de espalhamento frontal da partícula, já com a parte térmica fatorada. Vamos apresentar os resultados deste método no final desta seção, e os cálculos exatos estão no Apêndice D.3.

Um terceiro método, desenvolvido recentemente por Frenkel e colaboradores [49], diz que a parte térmica de um gráfico qualquer pode ser obtida pela simples aplicação de um operador à correspondente expressão do gráfico a temperatura nula ${ }^{4}$. Desta forma muitas das propriedades da teoria a temperatura finita podem ser estudadas diretamente através dos gráficos a temperatura nula.

Uma característica interessante em cálculos a temperatura finita é que o resultado pode depender de como tomamos alguns limites. O tensor de auto-energia por exemplo será uma função do momento externo $p$, e um limite de interesse é quando $p \rightarrow 0$. No entanto os cálculos mostram que, se tomarmos primeiro $p_{0}=0$ e depois $\vec{p} \rightarrow 0$, o resultado será diferente de tomarmos primeiro $\vec{p}=0$ e depois $p_{0} \rightarrow 0$. Isto mostra que o mesmo gráfico, quando calculado de diferentes maneiras para o momento externo tendendo a zero, possui diferentes valores quando calculado a temperatura finita. De fato isto acontece para todos os gráficos considerados aqui, e isto mostra que o tensor de auto-energia do fóton é não-analítico na origem do espaço dos momentos. É o exemplo mais direto que temos da chamada não-analiticidade quando fazemos cálculos a temperatura finita. No caso a temperatura nula, a invariância de Lorentz faz com que as amplitudes sejam funções analíticas com relação ao momento externo $p$. Já no caso a temperatura finita, a invariância de Lorentz é quebrada devido à escolha de um sistema de referência privilegiado, que é aquele especificado pelo quadrivetor $u^{\mu}$, que caracteriza a velocidade do reservatório térmico. Desta maneira as amplitudes dependem de $p^{0}$ e de $\vec{p}$ de maneira independente, portanto os limites acima considerados não precisam ser necessariamente os mesmos. O fato de os dois limites terem valores diferentes implica em efeitos físicos interessantes. De fato, o limite $p^{0}=0, \vec{p} \rightarrow 0$ corresponde a tomar o limite estático, o que dá origem a uma massa de blindagem para o campo elétrico no caso da QED usual. No caso da QED não-comutativa isto também acontece, como veremos mais adiante. Já o limite $\vec{p}=0, p^{0} \rightarrow 0$, por outro lado, dá origem a uma massa associada com o amortecimento das oscilações em um plasma, conhecida como plasmon, e as massas obtidas pelos diferentes limites não coincidem ${ }^{5}$.

ser feita exatamente em alguns limites.

${ }^{3}$ Conhecido na literatura como Forward Scattering Amplitude Method.

${ }^{4}$ Este método é conhecido como Thermal Operator Representation.

${ }^{5}$ Ver discussão na página 25 de [13]. 


\subsubsection{Soma sobre as freqüiências para a auto-energia do fóton}

Vamos utilizar aqui o primeiro método descrito acima. Os detalhes estão no Apêndice D.1, onde está feito o cálculo para cada um dos três primeiros diagramas da Figura 2.1 no limite estático, que correspondem aos diagramas que envolvem apenas os campos de gauge. O resultado para a parte dependente da temperatura é

$$
\begin{aligned}
& \Pi_{\mathrm{st}}^{\mu \nu}(p)= \Pi_{a \mathrm{st}}^{\mu \nu}(p)+\Pi_{b \mathrm{st}}^{\mu \nu}(p)+\Pi_{c \mathrm{st}}^{\mu \nu}(p) \\
&=-8 e^{2} \int \frac{\mathrm{d}^{3} k}{(2 \pi)^{3}} \sin ^{2}\left(\frac{k \times p}{2}\right) \frac{1}{|\vec{k}|}\left[n_{B}(|\vec{k}|) \eta^{\mu \nu}+\frac{n_{B}(|\vec{k}|)}{|\vec{k}|^{2}} k^{\mu} k^{\nu}-\frac{n_{B}^{\prime}(|\vec{k}|)}{|\vec{k}|} k^{\mu} k^{\nu}\right. \\
&\left.\quad-2 n_{B}(|\vec{k}|) \eta^{\mu 0} \eta_{0}^{\nu}\right]\left.\right|_{k^{0}=|\vec{k}|} .
\end{aligned}
$$

Note que o último termo só dará contribuição para a componente $\Pi^{00}$. Desta forma podemos calcular os fatores de forma (3.6), e por meio de (3.3) obter o tensor no limite estático no caso de 1-loop. Usando (3.6) e (3.9) obtemos

$$
\begin{aligned}
& \Pi_{\mathrm{L}}^{\mathrm{st}}=u_{\mu} u_{\nu} \Pi_{\mathrm{st}}^{\mu \nu} \\
&=8 e^{2} \int \frac{\mathrm{d}^{3} k}{(2 \pi)^{3}} \sin ^{2}\left(\frac{k \times p}{2}\right) n_{B}^{\prime}(|\vec{k}|) \\
&=-\frac{2}{3} e^{2} T^{2}+\frac{e^{2} T}{\pi|\overrightarrow{\tilde{p}}|} \operatorname{coth}(\pi|\overrightarrow{\tilde{p}}| T)-e^{2} T^{2} \operatorname{cossech}^{2}(\pi|\overrightarrow{\tilde{p}}| T) \\
& \tilde{\Pi}_{\mathrm{T}}^{\mathrm{st}}= \frac{\tilde{p}_{\mu} \tilde{p}_{\nu}}{\tilde{p}^{2}} \Pi_{\mathrm{st}}^{\mu \nu} \\
&=-8 e^{2} \int \frac{\mathrm{d}^{3} k}{(2 \pi)^{3}} \sin ^{2}\left(\frac{k \times p}{2}\right)\left[\frac{n_{B}(|\vec{k}|)}{|\vec{k}|}+\frac{(\tilde{p} \cdot k)^{2}}{\tilde{p}^{2}} \frac{n_{B}(|\vec{k}|)}{|\vec{k}|^{3}}-\frac{(\tilde{p} \cdot k)^{2}}{\tilde{p}^{2}} \frac{n_{B}^{\prime}(|\vec{k}|)}{|\vec{k}|^{2}}\right] \\
&=-\frac{2 e^{2}}{\pi^{2}|\overrightarrow{\tilde{p}}|^{2}}+\frac{e^{2} T}{\pi|\overrightarrow{\tilde{p}}|} \operatorname{coth}(\pi|\overrightarrow{\tilde{p}}| T)+e^{2} T^{2} \operatorname{cossech}^{2}(\pi|\overrightarrow{\tilde{p}}| T) \\
& \Pi_{\mathrm{T}}^{\mathrm{st}}=\left(\eta_{\mu \nu}-\frac{p_{\mu} p_{\nu}}{p^{2}}\right) \Pi_{\mathrm{st}}^{\mu \nu}-\Pi_{\mathrm{L}}^{\mathrm{st}}-\tilde{\Pi} \mathrm{I}_{\mathrm{T}}^{\mathrm{st}} \\
&=0
\end{aligned}
$$


As integrais estão feitas no Apêndice D.2. O tensor de auto-energia do fóton no limite estático é dado então por

$$
\Pi_{\mathrm{st}}^{\mu \nu}=u^{\mu} u^{\nu} \Pi_{\mathrm{L}}^{\mathrm{st}}+\frac{\tilde{p}^{\mu} \tilde{p}^{\nu}}{\tilde{p}^{2}} \tilde{\Pi}_{\mathrm{T}}^{\mathrm{st}}
$$

Vamos estudar o comportamento de $\Pi_{\mathrm{st}}^{\mu \nu}$ em dois limites diferentes. Observe que o argumento das funções hiperbólicas nas expressões (3.10) e (3.11) é dado por $\tau \equiv \pi|\overrightarrow{\tilde{p}}| T=\pi \theta \bar{p} T$, onde escolhemos um sistema de coordenadas onde $\theta_{12}=-\theta_{21}=\theta$ são as únicas componentes não nulas do parâmetro de nãocomutatividade, ou seja, $\theta_{3 i}=0$, e renomeamos $\vec{p}^{2}=|\vec{p}|^{2} \equiv \bar{p}^{2}$. Desta forma obtemos para as expressões (3.10) e (3.11):

$$
\begin{aligned}
& \Pi_{\mathrm{L}}^{\mathrm{st}}=-\frac{2}{3} e^{2} T^{2}+\frac{e^{2} T^{2}}{\tau} \operatorname{coth}(\tau)-e^{2} T^{2} \operatorname{cossech}^{2}(\tau) \\
& \tilde{\Pi}_{\mathrm{T}}^{\mathrm{st}}=-\frac{2 e^{2} T^{2}}{\tau^{2}}+\frac{e^{2} T^{2}}{\tau} \operatorname{coth}(\tau)+e^{2} T^{2} \operatorname{cossech}^{2}(\tau) .
\end{aligned}
$$

No limite $\pi \theta \bar{p} T<<1$ ou $\tau<<1$, podemos usar as expansões das funções hiperbólicas dadas no Apêndice C.3, de forma que obtemos

$$
\begin{aligned}
& \Pi_{\mathrm{L}}^{\mathrm{st}} \simeq-\frac{4}{45} e^{2} T^{2}(\pi \theta \bar{p} T)^{2} \\
& \tilde{\Pi}_{\mathrm{T}}^{\mathrm{st}} \simeq-\frac{1}{45} e^{2} T^{2}(\pi \theta \bar{p} T)^{2} .
\end{aligned}
$$

No limite oposto, ou seja, $\pi \theta \bar{p} T>>1$ ou $\tau>>1$, podemos usar a forma assintótica das funções hiperbólicas

$$
\lim _{\tau \rightarrow \infty} \operatorname{coth}(\tau)=1 \quad \lim _{\tau \rightarrow \infty} \operatorname{cossech}(\tau)=0
$$

de forma que obtemos

$$
\begin{aligned}
& \Pi_{\mathrm{L}}^{\mathrm{st}} \simeq-\frac{2}{3} e^{2} T^{2} \\
& \tilde{\Pi}_{\mathrm{T}}^{\mathrm{st}} \simeq 0 .
\end{aligned}
$$

Portanto, o tensor de auto-energia no limite estático se comporta de duas maneiras diferentes em ordem dominante,

$$
\Pi_{\mathrm{st}}^{\mu \nu} \sim \begin{cases}e^{2} T^{2}(\pi \theta \bar{p} T)^{2} & (\pi \theta \bar{p} T<<1) \\ e^{2} T^{2} & (\pi \theta \bar{p} T>>1) .\end{cases}
$$

Vemos que no limite $(\pi \theta \bar{p} T)^{2}>>1$ o tensor de auto-energia se comporta como $e^{2} T^{2}$, portanto da mesma ordem da contribuição do loop fermiônico, conforme resultado (1.114). Se o processo ocorrer a uma tem- 
peratura menor, o fator $(\pi \theta \bar{p} T)^{2}<<1$ vai suprimir o comportamento $e^{2} T^{2}$, de forma que só a parte fermiônica contribui. É interessante notar também que no limite de altíssimas temperaturas não há dependência com o parâmetro de não comutatividade para o tensor.

\subsection{Blindagem dos campos elétrico e magnético}

Da mesma forma que no caso da eletrodinâmica comutativa a temperatura finita estudada na seção 1.2.5, podemos ter também uma contribuição para os raios de blindagem (raio de Debye) dos campos elétrico e magnético vindos da parte não-comutativa da teoria.

Como já vimos, as massas elétrica e magnética são definidas como os inversos do raio de Debye para as partes longitudinal e transversal, respectivamente, do propagador. Aqui no caso não-comutativo temos o aparecimento de três pólos físicos, ao contrário do caso comutativo com dois. Estes pólos físicos estão presentes no denominador do propagador (3.8), e a eles estão associadas 3 massas diferentes. Para entendermos como se definem essas massas, lembre-se que uma partícula relativística de massa $m$ deve satisfazer a equação de energia $p^{2}=p_{0}^{2}-\vec{p}^{2}=m^{2}$. No caso do fóton a temperatura zero, esta massa é nula, o que significa que o alcance do campo elétrico é infinito. No entanto, devido a efeitos térmicos e não-comutativos, o fóton pode adquirir uma massa efetiva dentro de um meio, o que o torna uma partícula de alcance finito, levando ao amortecimento dos campos eletromagnéticos (blindagem). O propagador exato do fóton deve levar isto em consideração, por isso a presença dos três fatores no denominador da expressão (3.8). Se algum deles for diferente de zero, significa que os fótons correspondentes (longitudinais ou transversais) estão adquirindo uma massa efetiva, levando ao amortecimento da respectiva componente do campo.

Para definirmos estas massas, basta substituir $p^{2}=m^{2}$ no denominador correspondente. Portanto, aos três denominadores do propagador (3.8), podemos definir as seguintes massas no limite estático ${ }^{6}\left(p_{0}=0\right.$, $\vec{p} \rightarrow 0):$

$$
\begin{gathered}
m_{\mathrm{mag}}^{2} \equiv+\Pi_{\mathrm{T}}^{\mathrm{st}}(0, \vec{p}) \\
m_{\mathrm{el}}^{2} \equiv+\Pi_{\mathrm{L}}^{\mathrm{st}}(0, \vec{p}) \\
\tilde{m}_{\mathrm{mag}}^{2} \equiv+\tilde{\Pi}_{\mathrm{T}}^{\mathrm{st}}(0, \vec{p}) .
\end{gathered}
$$

A primeira é a massa magnética, associada à parte transversal do propagador, que já apareceu no caso comutativo, sendo nula naquele caso. A segunda é a massa elétrica, associada à parte longitudinal do propagador, e também apareceu no caso comutativo, conforme já vimos anteriormente ${ }^{7}$. A terceira é uma nova componente, podemos chamá-la de massa magnética não-comutativa, pois ela só aparece aqui na

\footnotetext{
${ }^{6}$ Note que ainda existe uma dependência com $\vec{p}$ vinda dos vértices não-comutativos, mas já foi tomado o limite $p<<k$ no cálculo do tensor.

${ }^{7}$ Lembre-se que estas duas massas se deviam a um loop fermiônico no caso comutativo da seção 1.2.5, sendo que aqui, se elas existirem, devem-se somente à não-comutatividade da teoria, que dá origem a outros diagramas de 1-loop, que não têm nada a ver com o fermiônico.
} 
teoria não-comutativa. Vamos ver quais as contribuições para estas massas devido à não-comutatividade.

Vamos começar pela massa elétrica (3.19), que corresponde ao pólo do segundo termo do propagador (3.8). A singularidade acontece quando

$$
p^{2}-\Pi_{\mathrm{L}}=0 .
$$

No limite estático isto corresponde a

$$
\vec{p}^{2}=-\Pi_{\mathrm{L}}^{\mathrm{st}}(0, \vec{p})
$$

Observe que esta expressão depende do momento externo $\vec{p}$ do lado direito, portanto a condição (3.21) será satisfeita quando

$$
\vec{p}^{2}+\Pi_{\mathrm{L}}^{\mathrm{st}}(0, \vec{p})=0
$$

Em geral esta equação não é fácil de se resolver, mesmo que já tenhamos tomado o limite $p<<k$. Ainda existe uma contribuição de $\vec{p}$ vinda de $\overrightarrow{\tilde{p}}$ no lado direito, onde cada componente de $\overrightarrow{\tilde{p}}$ se relaciona a $\vec{p}$ por $\tilde{p}^{i}=\theta^{i j} p_{j}$. A equação (3.23) então fica, usando (3.10) e a mesma parametrização adotada anteriormente, onde $\pi|\overrightarrow{\tilde{p}}| T=\pi \theta \bar{p} T$,

$$
\bar{p}^{2}-\frac{2}{3} e^{2} T^{2}+\frac{e^{2} T}{\pi \theta \bar{p}} \operatorname{coth}(\pi \theta \bar{p} T)-e^{2} T^{2} \operatorname{cossech}^{2}(\pi \theta \bar{p} T)=0 .
$$

A pergunta é: para qual valor de temperatura $T$ esta equação tem solução para algum valor real de $\bar{p}$ ? A solução só pode ser obtida numericamente. Uma forma é fazer o gráfico desta equação e ver onde ela cruza o eixo horizontal. Para este valor de $\bar{p}$ a temperatura corresponde à temperatura crítica que estamos procurando. Alguns gráficos estão plotados na Figura 3.1, para diversos valores de $T$ e com $\theta=10^{-6}$. Percebemos destes gráficos que tanto para $T=1026$ como para $T=1033$ não há solução para a equação (3.24). Já para o valor $T=1037$ começa a ficar evidente a existência de uma solução, que é óbvia para $T=1043$ e valores superiores. Desta forma a temperatura crítica $T_{c}$ para que exista uma solução da equação deve estar entre $T=1033$ e $T=1037$. O valor quase exato de $T_{c}$ pode ser encontrado da seguinte maneira. Note que, com os parâmetros utilizados, o momento $\bar{p}$ para o qual existe solução é da ordem de 100 (gráfico (c)). Para este valor do momento e da temperatura correspondente, o argumento das funções hiperbólicas é dado por $\pi \theta \bar{p} T \simeq 0,33$, portanto $\pi \theta \bar{p} T<1$, de forma que podemos expandir as funções hiperbólicas usando as séries dadas no Apêndice C.3. Obtemos assim para a equação (3.24) a seguinte expressão:

$$
\bar{p}^{2}-\frac{4}{45} e^{2} \pi^{2} \theta^{2} \bar{p}^{2} T^{4}=0,
$$




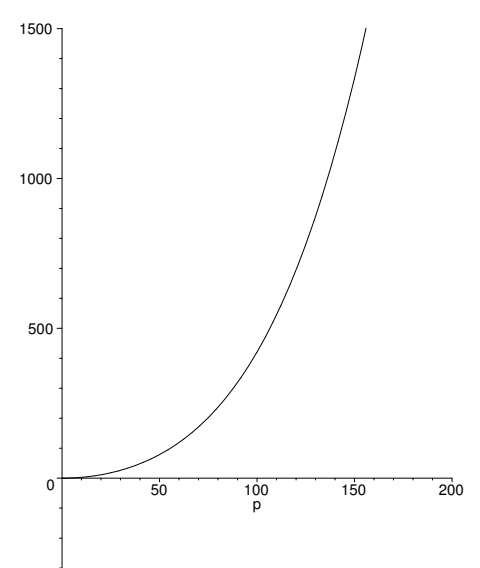

(a)

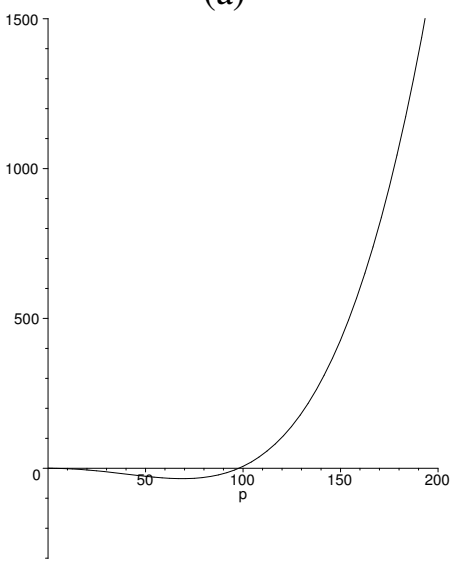

(c)

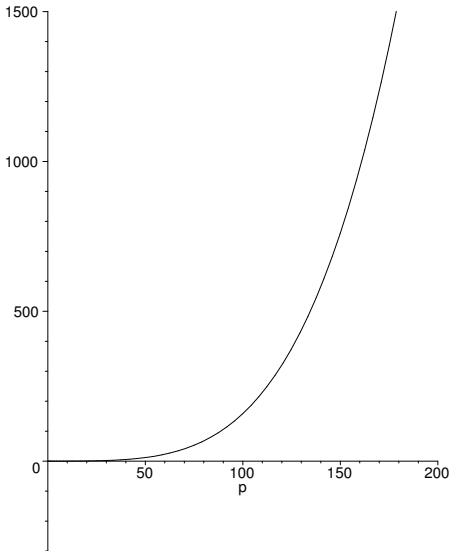

(b)

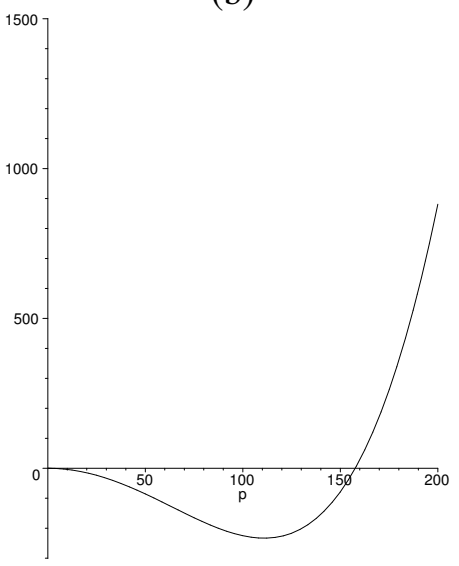

(d)

Figura 3.1: Gráficos da função dada no lado esquerdo da eq. (3.24) como função do momento $\bar{p}$. Adotamos $e=1$ e $\theta=10^{-6}$. Os valores de $T$ são: (a) $T=1026$; (b) $T=1033$; (c) $T=1037$; (d) $T=1043$.

que fornece diretamente o valor da temperatura crítica

$$
T_{\mathrm{c}}^{2}=\frac{3 \sqrt{5}}{2 \pi e \theta}
$$

acima da qual existirá uma solução real da equação (3.24), e portanto, uma massa elétrica. Para o valor de $\theta$ dado acima, $T_{c} \simeq 1033,27$, em perfeito acordo com o que foi discutido acima. No sistema de unidades onde $c=\hbar=k_{B}=1$, a dimensão de $\theta$ é [eV] ${ }^{-2}$ e da temperatura é [eV]. Na verdade, estima-se que o valor do parâmetro $\theta$ deva ser da ordem de $(10 \mathrm{TeV})^{-2}$, o que leva a uma temperatura crítica da ordem ${ }^{8}$ de $10^{17} \mathrm{~K}$, portanto uma temperatura muitíssimo alta.

Vamos estudar agora a massa magnética não-comutativa, vinda da equação (3.20). Usando o resultado (3.11) para a contribuição do tensor, a equação que deve ser satisfeita para a existência de um pólo no denominador do terceiro termo do propagador (3.8) é

$$
\bar{p}^{2}-\frac{2 e^{2}}{\pi^{2} \theta^{2} \bar{p}^{2}}+\frac{e^{2} T}{\pi \theta \bar{p}} \operatorname{coth}(\pi \theta \bar{p} T)+e^{2} T^{2} \operatorname{cossech}^{2}(\pi \theta \bar{p} T)=0 .
$$

\footnotetext{
${ }^{8}$ Usamos $1 \mathrm{GeV} \sim 10^{13} \mathrm{~K}$.
} 
Um gráfico típico desta função está representado na Figura 3.2, onde podemos perceber que esta equação não tem solução real, ou seja, ela não cruza o eixo horizontal para nenhum valor real de $\bar{p}$. Isto mostra que não há a geração de uma massa magnética associada à parte não-comutativa da teoria. Não há blindagem para os campos magnéticos neste caso.

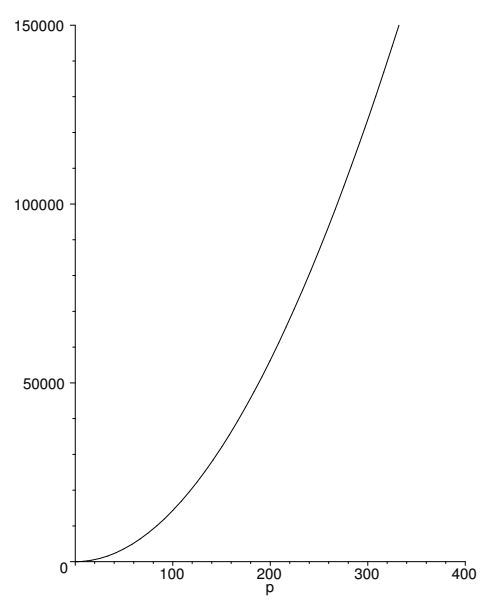

Figura 3.2: Gráfico da função dada no lado esquerdo de (3.27) como função do momento $\bar{p}$. Adotamos $e=1, \theta=10^{-6}$ e $T=1000$.

Para a massa magnética da equação (3.18), pelo resultado (3.12) fica claro que também não há blindagem associada a este termo, assim como no caso da QED comutativa.

Portanto, o único efeito de blindagem que aparece da parte não-comutativa é a blindagem do campo elétrico, que de fato já existe no caso comutativo. No entanto somente a altíssimas temperaturas este efeito é significativo.

No que segue estaremos interessados em estudar a ação efetiva em 1-loop desta teoria no limite de altas temperaturas e no limite estático, e para isso vamos precisar das amplitudes de $n$-pontos em 1-loop, dadas nas seções seguintes.

\subsection{Amplitude de 3-pontos}

No estudo da amplitude de 2 pontos (auto-energia do fóton) obtivemos o resultado geral que $\Pi_{\mathrm{st}}^{00} \neq 0$, $\Pi_{\mathrm{st}}^{i j} \neq 0$ e $\Pi_{\mathrm{st}}^{0 i}=\Pi_{\mathrm{st}}^{i 0}=0$. Esta característica também é obtida no caso da amplitude de 3 ou mais pontos, ou seja, as amplitudes com um número ímpar de índices temporais se anulam, e cálculos explícitos mostram isso. Neste caso, para a amplitude de 3-pontos temos

$$
\Gamma_{\mathrm{st}}^{000}=0=\Gamma_{\mathrm{st}}^{0 i j}
$$

e precisamos calcular apenas $\Gamma_{\mathrm{st}}^{00 i} \mathrm{e} \Gamma_{\mathrm{st}}^{i j k}$. Os diagramas de 1-loop que contribuem para a amplitude de 3 pontos da QED não-comutativa estão representados na Figura 3.3. 


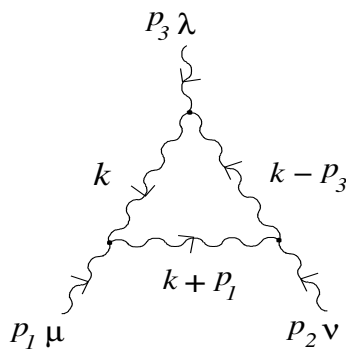

(a)

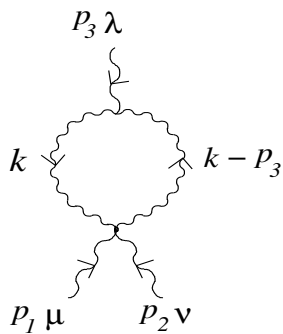

(d)

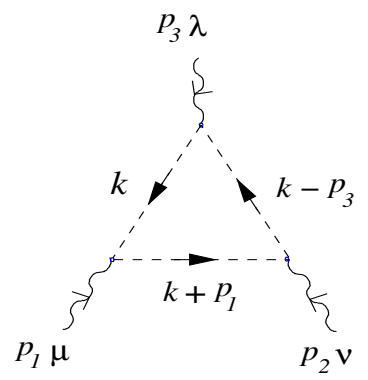

(b)

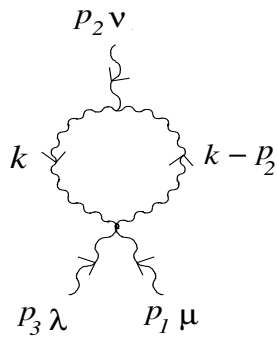

(e)

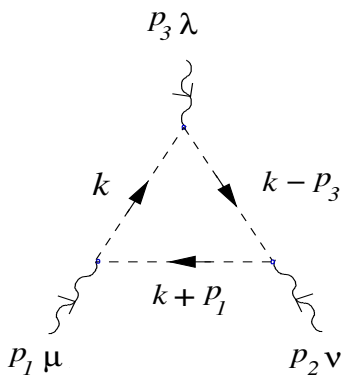

(c)

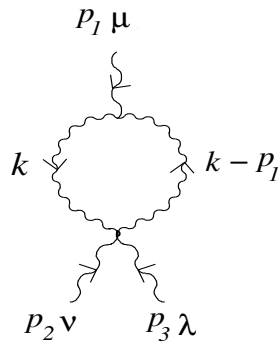

(f)

Figura 3.3: Diagramas de 1-loop que contribuem para a amplitude de 3-pontos.

Das discussões anteriores vimos que as contribuições dominantes para a amplitude vieram, no limite estático, dos termos de ordem mais baixa no momento externo. Isto corresponde a tomar os momentos externos iguais a zero, exceto no fator trigonométrico de vértice, que "carrega" a informação da nãocomutatividade.

Devido à simetria das amplitudes na ordem dominante, concluímos que qualquer amplitude com um número ímpar de índices temporais se anula, o caso mais trivial sendo $\Pi_{\mathrm{st}}^{0 i}=0$ obtido anteriormente. Para a amplitude de três pontos obtemos que

$$
\Gamma_{\mathrm{st}}^{000}=0=\Gamma_{\mathrm{st}}^{0 i j}
$$

e precisamos calcular apenas $\Gamma_{\mathrm{st}}^{00 i}$ e $\Gamma_{\mathrm{st}}^{i j k}$.

Para o caso da amplitude de 2-pontos temos que o termo dominante da amplitude corresponde a um termo de grau zero no momento externo no integrando, exceto pelo fator trigonométrico, que é linear no momento externo. Aqui esta mesma característica pode ser observada, pois a contribuição quadrática no momento externo no fator trigonométrico de vértice pode ser desprezada com relação à contribuição linear, pois $p<<k$. Desta forma, um fator trigonométrico típico será da forma (ver Figura 3.3(a) por exemplo)

$$
\begin{aligned}
\sin \left(\frac{k \times p_{1}}{2}\right) \sin & \left(\frac{\left(k-p_{3}\right) \times p_{2}}{2}\right) \sin \left(\frac{k \times p_{3}}{2}\right) \\
& \approx \sin \left(\frac{k \times p_{1}}{2}\right) \sin \left(\frac{k \times p_{2}}{2}\right) \sin \left(\frac{k \times p_{3}}{2}\right)
\end{aligned}
$$


Isto corresponde a usar a aproximação

$$
\theta p^{2}<<1
$$

onde $p$ representa a magnitude típica do momento externo. Como os fatores trigonométricos não dependem da componente temporal $\left(\theta^{0 i}=0\right)$, os cálculos das amplitudes de ordens mais altas simplificam-se enormemente no limite estático, e podem ser feitos diretamente pela soma sobre as freqüências de Matsubara. Os cálculos explícitos mostram que, quando todos os gráficos para uma determinada amplitude são somados, os fatores trigonométricos correspondentes a uma amplitude de $n$-pontos podem ser escritos como o produto de $n$ fatores da forma $\sin \left(k \times p_{i} / 2\right)$, com $i=1,2, \cdots, n$. Isto está de acordo com a simetria esperada da amplitude total.

Seguindo esta "receita" para o cálculo da amplitude, é fácil ver que a correspondente amplitude de 3-pontos pode ser escrita como

$$
\begin{aligned}
\Gamma_{\mu \nu \lambda}^{\mathrm{st}}= & i e^{3} T \int \frac{\mathrm{d}^{3} k}{(2 \pi)^{3}} \sin \left(\frac{k \times p_{1}}{2}\right) \sin \left(\frac{k \times p_{2}}{2}\right) \sin \left(\frac{k \times p_{3}}{2}\right) \sum_{n}\left[\frac{128 k_{\mu} k_{\nu} k_{\lambda}}{\left[(2 \pi n T)^{2}+k^{2}\right]^{3}}\right. \\
& \left.-\frac{32}{\left[(2 \pi n T)^{2}+k^{2}\right]^{2}}\left(\delta_{\mu \nu} k_{\lambda}+\delta_{\lambda \mu} k_{\nu}+\delta_{\nu \lambda} k_{\mu}\right)\right] .
\end{aligned}
$$

Não vamos fazer aqui os cálculos explícitos da amplitude de 3-pontos. O que queremos é encontrar as componentes não nulas $\Gamma^{00 i}$ e $\Gamma^{i j k}$ no limite estático. Os detalhes estão no Apêndice E.1. Os resultados são:

$$
\begin{aligned}
& \Gamma_{00 i}^{\mathrm{st}}\left(p_{1}, p_{2}, p_{3}\right)=i e\left[\tilde{p}_{1, i} \Pi_{00}^{\mathrm{st}}\left(p_{1}\right)+\tilde{p}_{2, i} \Pi_{00}^{\mathrm{st}}\left(p_{2}\right)+\tilde{p}_{3, i} \Pi_{00}^{\mathrm{st}}\left(p_{3}\right)\right], \\
& \Gamma_{i j k}^{\mathrm{st}}\left(p_{1}, p_{2}, p_{3}\right)=i e\left[\tilde{p}_{1, k} \Pi_{i j}^{\mathrm{st}}\left(p_{1}\right)+\tilde{p}_{2, k} \Pi_{i j}^{\mathrm{st}}\left(p_{2}\right)+\tilde{p}_{3, k} \Pi_{i j}^{\mathrm{st}}\left(p_{3}\right)\right],
\end{aligned}
$$

de forma que o conhecimento da auto-energia do fóton $\Pi_{\mathrm{st}}^{\mu \nu}$ é suficiente para calcularmos também a amplitude de 3-pontos no limite estático.

\subsection{Amplitude de 4-pontos}

De maneira semelhante ao caso da função de 3-pontos, as componentes $\Gamma_{\mathrm{st}}^{000 i}$ e $\Gamma_{\mathrm{st}}^{0 i j k}$ são nulas. Além disto, assim como $\Pi_{\mathrm{st}}^{00}$ representa uma componente independente, também temos que $\Gamma_{\mathrm{st}}^{0000}$ deve ser calculada independentemente, pois ela dará contribuição para a amplitude de 5-pontos, mas vamos nos concentrar aqui somente nas componentes espaciais.

Alguns dos diagramas que contribuem para a amplitude de 4-pontos em 1-loop da QED não-comutativa são mostrados na Figura 3.4. Não estão representados os diagramas que envolvem ghosts. 


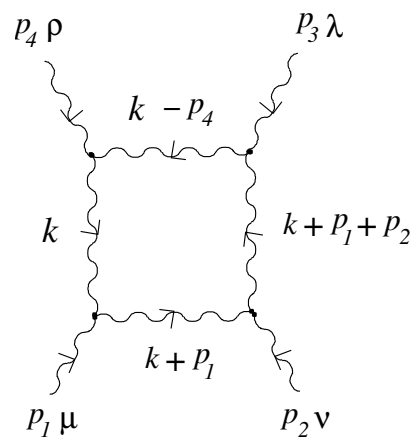

(a)

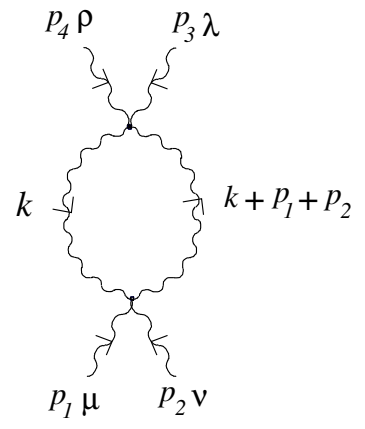

(b)

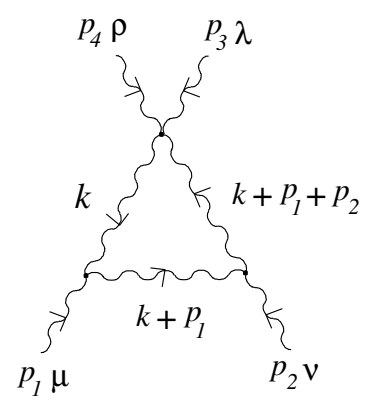

(c)

Figura 3.4: Alguns diagramas de 1-loop que contribuem para a amplitude de 4-pontos. Não estão representados diagramas que envolvem ghosts.

A amplitude de 4-pontos no limite estático será

$$
\begin{aligned}
& \Gamma_{\mu \nu \lambda \rho}^{\mathrm{st}}\left(p_{1}, p_{2}, p_{3}, p_{4}\right) \\
& =32 e^{4} \int \frac{\mathrm{d}^{3} k}{(2 \pi)^{3}} \sin \left(\frac{k \times p_{1}}{2}\right) \sin \left(\frac{k \times p_{2}}{2}\right) \sin \left(\frac{k \times p_{3}}{2}\right) \sin \left(\frac{k \times p_{4}}{2}\right) \\
& \quad \times T \sum_{n}\left[\frac{24 k_{\mu} k_{\nu} k_{\lambda} k_{\rho}}{\left((2 \pi n T)^{2}+k^{2}\right)^{4}}-\frac{4}{\left[(2 \pi n T)^{2}+k^{2}\right]^{3}}\left(\delta_{\mu \nu} k_{\lambda} k_{\rho}+\delta_{\rho \mu} k_{\nu} k_{\lambda}+\delta_{\lambda \rho} k_{\mu} k_{\nu}+\delta_{\nu \lambda} k_{\rho} k_{\mu}\right)\right. \\
& \left.\quad+\frac{2}{\left[(2 \pi n T)^{2}+k^{2}\right]^{2}}\left(\delta_{\mu \nu} \delta_{\lambda \rho}+\delta_{\rho \mu} \delta_{\nu \lambda}\right)\right]
\end{aligned}
$$

Escrita em termos das amplitudes de 3-pontos, a amplitude com componentes puramente espaciais é (os detalhes desta dedução estão no Apêndice E.2)

$$
\begin{gathered}
\Gamma_{i j k l}^{\mathrm{st}}\left(p_{1}, p_{2}, p_{3}, p_{4}\right)=i e\left[\tilde{p}_{1, l} \Gamma_{i j k}^{\mathrm{st}}\left(p_{1}+p_{4}, p_{2}, p_{3}\right)+\tilde{p}_{2, l} \Gamma_{i j k}^{\mathrm{st}}\left(p_{1}, p_{2}+p_{4}, p_{3}\right)\right. \\
\left.+\tilde{p}_{3, l} \Gamma_{i j k}^{\mathrm{st}}\left(p_{1}, p_{2}, p_{3}+p_{4}\right)\right]
\end{gathered}
$$

\subsection{Identidades de Ward}

Dos resultados apresentados nas seções anteriores vemos que as amplitudes de 3 e 4-pontos satisfazem a identidades de Ward simples e podem ser completamente determinadas a partir da auto-energia do fóton, exceto quando todas as componentes das amplitudes são temporais. E para o caso de um número ímpar de índices temporais, as amplitudes são nulas. 
Para a amplitude de 3-pontos podemos facilmente verificar que

$$
\begin{gathered}
p_{3, i} \Gamma_{00 i}^{\mathrm{st}}\left(p_{1}, p_{2}, p_{3}\right)=i e\left[p_{3} \cdot \tilde{p}_{1} \Pi_{00}^{\mathrm{st}}\left(p_{1}\right)+p_{3} \cdot \tilde{p}_{2} \Pi_{00}^{\mathrm{st}}\left(p_{2}\right)\right] \\
p_{3, k} \Gamma_{i j k}^{\mathrm{st}}\left(p_{1}, p_{2}, p_{3}\right)=i e\left[p_{3} \cdot \tilde{p}_{1} \Pi_{i j}^{\mathrm{st}}\left(p_{1}\right)+p_{3} \cdot \tilde{p}_{2} \Pi_{i j}^{\mathrm{st}}\left(p_{2}\right)\right]
\end{gathered}
$$

e para a amplitude de 4-pontos apenas com componentes espaciais temos

$$
\begin{aligned}
p_{4, l} \Gamma_{i j k l}^{\mathrm{st}}\left(p_{1}, p_{2}, p_{3}, p_{4}\right)= & i e\left[\left(\tilde{p}_{1} \cdot p_{4}\right) \Gamma_{i j k}^{\mathrm{st}}\left(p_{1}+p_{4}, p_{2}, p_{3}\right)+\left(\tilde{p}_{2} \cdot p_{4}\right) \Gamma_{i j k}^{\mathrm{st}}\left(p_{1}, p_{2}+p_{4}, p_{3}\right)\right. \\
& \left.+\left(\tilde{p}_{3} \cdot p_{4}\right) \Gamma_{i j k}^{\mathrm{st}}\left(p_{1}, p_{2}, p_{3}+p_{4}\right)\right] .
\end{aligned}
$$

Fica claro desta discussão que, no limite estático, as amplitudes de 3 e 4-pontos satisfazem a identidades de Ward, pelo menos quando nem todos os índices são temporais, e isto segue de uma invariância da teoria por transformações de gauge no limite estático. Dessa forma, estas amplitudes podem ser relacionadas recursivamente, todas escritas em termos do tensor de auto-energia. $\mathrm{O}$ caso da componente $\Gamma_{0000}^{\mathrm{st}}$ da função de 4-pontos é diferente, ela não pode ser expressa por meio da correspondente função de 3-pontos, pois esta se anula. Desta forma, e no caso geral, uma amplitude com todas as componentes temporais não pode ser relacionada a uma amplitude de ordem inferior, elas precisam ser calculadas individualmente, e em geral não se anulam, exceto no caso de um número ímpar de componentes. Portanto, para o cálculo da amplitude $\Gamma_{0000 i}^{\text {st }}$ por exemplo, precisaríamos do conhecimento da correspondente amplitude de 4-pontos. Desta forma não é possível estabelecer uma ação efetiva que gere todas as amplitudes. Mas isto pode ser feito para as amplitudes que envolvam apenas índices espaciais, e é isto que faremos na seção seguinte.

\subsection{Ação efetiva}

A análise anterior mostrou que todas as componentes não triviais da amplitude de 3-pontos podem ser determinadas do conhecimento da auto-energia no limite estático. No entanto, para a amplitude de 4-pontos precisamos calcular explicitamente a componente $\Gamma_{0000}^{\text {st }}$, pois ela não está relacionada à amplitude de ordem inferior. Esta componente, entretanto, será importante para o completo conhecimento da amplitude de 5pontos. De fato, para toda amplitude de ordem par, teremos uma estrutura independente, que não se relaciona à de ordem inferior, o que leva a uma impossibilidade de se obter a ação efetiva completa. Por outro lado, como vimos, as componentes das amplitudes apenas com índices espaciais são relacionadas recursivamente a amplitudes de ordem inferior, satisfazendo a identidades de Ward simples. Podemos então tentar obter a parte da ação efetiva que dependa apenas das componentes espaciais dos campos de gange, ou seja, de $A_{i}$.

Vamos representar por $\Gamma\left[A_{i}\right]$ a parte da ação efetiva a altas temperaturas que dependa somente das componentes espaciais do campo de gauge. A invariância por uma transformação gauge infinitesimal no 
limite estático leva à identidade de Ward

$$
\frac{\delta \Gamma\left[A_{k}\right]}{\delta \omega(x)}=\int \mathrm{d} y \frac{\delta A_{i}(y)}{\delta \omega(x)} \frac{\delta \Gamma\left[A_{k}\right]}{\delta A_{i}(y)}=D_{i} \frac{\delta \Gamma\left[A_{k}\right]}{\delta A_{i}(x)}=0
$$

onde $\omega(\vec{x})$ representa o parâmetro infinitesimal da transformação, e depende somente das coordenadas espaciais. Vista de outra forma, a equação (3.39) pode ser interpretada como a conservação covariante da corrente

$$
D_{i} j^{i}\left[A_{k}\right]=0 \text {. }
$$

$\mathrm{Na}$ aproximação $\theta p^{2}<<1$ que estamos interessados, a derivada covariante (2.52) toma a seguinte forma ${ }^{9}$

$$
D_{i}=\partial_{i}+e\left(\partial_{j} A_{i}\right) \tilde{\partial}_{j}
$$

Com isto, a conservação da corrente (3.39) fica

$$
\partial_{i} \frac{\delta \Gamma\left[A_{k}\right]}{\delta A_{i}}+e\left(\partial_{j} A_{i}\right) \tilde{\partial}_{j} \frac{\delta \Gamma\left[A_{k}\right]}{\delta A_{i}}=0
$$

ou

$$
\partial_{i}\left(\frac{\delta \Gamma\left[A_{k}\right]}{\delta A_{i}}+e A_{j} \tilde{\partial}_{i} \frac{\delta \Gamma\left[A_{k}\right]}{\delta A_{j}}\right)=0 .
$$

Isto determina que a quantidade entre parênteses representa uma quantidade que se conserva, sendo transversal à derivada $\partial_{i}$. Podemos representar esta quantidade por

$$
\left(\delta_{i j}+e A_{j} \tilde{\partial}_{i}\right) \frac{\delta \Gamma\left[A_{k}\right]}{\delta A_{j}}=X_{i}^{\mathrm{T}},
$$

de maneira que

$$
\partial_{i} X_{i}^{\mathrm{T}}=0
$$

Tomando a derivada funcional de (3.44) com relação a $A_{j}$, obtemos

$$
\begin{aligned}
\frac{\delta}{\delta A_{j}} X_{i}^{\mathrm{T}} & =\frac{\delta}{\delta A_{j}}\left(\delta_{i k}+e A_{k} \tilde{\partial}_{i}\right) \frac{\delta \Gamma\left[A_{j}\right]}{\delta A_{k}} \\
& =\frac{\delta^{2} \Gamma\left[A_{j}\right]}{\delta A_{i} \delta A_{j}}+e \tilde{\partial}_{i} \frac{\delta \Gamma\left[A_{j}\right]}{\delta A_{j}}+e A_{k} \tilde{\partial}_{i} \frac{\delta^{2} \Gamma\left[A_{j}\right]}{\delta A_{k} \delta A_{j}}
\end{aligned}
$$

Em primeira ordem (ordem $e=0$ )

$$
\frac{\delta}{\delta A_{j}} X_{i}^{\mathrm{T}} \approx \frac{\delta^{2} \Gamma\left[A_{j}\right]}{\delta A_{i} \delta A_{j}} \equiv \Pi_{i j}^{\mathrm{st}},
$$

\footnotetext{
${ }^{9}$ Basta expandir o produto Moyal e tomar somente o termo linear em $\theta$.
} 
que como sabemos é a definição para a função de 2-pontos (auto-energia) derivada da ação efetiva $\Gamma\left[A_{j}\right]$. Desta forma, em primeira ordem temos

$$
X_{i}^{\mathrm{T}(0)}=\Pi_{i j}^{\mathrm{st}} A_{j}
$$

É claro que $X_{i}^{\mathrm{T}}$ contém termos de ordens mais altas nos campos, como podemos ver de (3.46). No entanto, pode-se mostrar que tomar derivadas funcionais de ordens mais altas em (3.44) corresponde a fazer uma simetrização dos índices das amplitudes de ordens mais altas. Tendo esta simetrização em mente, podemos descartar as contribuições vindas dos termos com ordens mais altas nos campos em $X_{i}^{\mathrm{T}}$. Podemos então resolver a equação (3.39) para a corrente, e isto é feito verificando-se que o inverso do termo entre parênteses em (3.44) é

$$
\left(\delta_{i j}+e A_{j} \tilde{\partial}_{i}\right)^{-1}=\delta_{i j}-e A_{j} \tilde{\partial}_{i}+e^{2} A_{k} \tilde{\partial}_{i} A_{j} \tilde{\partial}_{k}-e^{3} A_{k} \tilde{\partial}_{i} A_{l} \tilde{\partial}_{k} A_{j} \tilde{\partial}_{l}+\cdots
$$

de maneira que

$$
\frac{\delta \Gamma\left[A_{k}\right]}{\delta A_{i}}=\left(\delta_{i j}+e A_{j} \tilde{\partial}_{i}\right)^{-1} X_{j}^{\mathrm{T}(0)}
$$

Além disso, da definição da derivada covariante (2.52) podemos ver que

$$
\partial_{j}\left(\delta_{j i}+e A_{i} \tilde{\partial}_{j}\right) \simeq D_{i}
$$

de maneira que podemos escrever

$$
\left(\delta_{i j}+e A_{j} \tilde{\partial}_{i}\right)^{-1} \simeq D_{j}^{-1} \partial_{i}
$$

Usando (3.52), podemos determinar a corrente (3.50) como sendo

$$
j_{i}\left[A_{k}\right]=\frac{\delta \Gamma\left[A_{k}\right]}{\delta A_{i}}=D_{j}^{-1} \partial_{i} \Pi_{j k}^{\mathrm{st}} A_{k}
$$

Esta corrente se conserva covariantemente, pois a auto-energia é transversal, e além disso esta expressão leva corretamente às amplitudes de $n$-pontos, como pode ser verificado explicitamente ${ }^{10}$. Vamos mostrar brevemente como se obtém as amplitudes de 2 e 3-pontos. A amplitude de 2-pontos é obtida trivialmente da (3.53) por meio de

$$
\left.\frac{\delta j_{i}\left[A_{k}\right]}{\delta A_{l}}\right|_{A=0}=\left.\frac{\delta^{2} \Gamma\left[A_{k}\right]}{\delta A_{l} \delta A_{i}}\right|_{A=0}
$$

Para a amplitude de 3-pontos, sem levar em conta a simetrização dos momentos, devemos tomar a forma completa de $X_{i}^{\mathrm{T}}$ dada na (3.46). Usando as expressões (3.46) e (3.49-3.53), a amplitude de 3-pontos virá do termo linear em "e" de

$$
\left.\frac{\delta^{2} j_{i}\left[A_{k}\right]}{\delta A_{l} \delta A_{m}}\right|_{A=0}=\left.\frac{\delta^{2}}{\delta A_{l} \delta A_{m}}\left(\delta_{i j}-e A_{j} \tilde{\partial}_{i}\right)\left(\Pi_{j k}^{\mathrm{st}} A_{k}+e A_{n} \tilde{\partial}_{j} \frac{\delta \Gamma}{\delta A_{n}}\right)\right|_{A=0} .
$$

\footnotetext{
${ }^{10}$ Para isso deve-se levar em conta a simetrização dos momentos externos $p_{i}$.
} 
No espaço dos momentos (escrevendo os campos $A$ 's como transformadas de Fourier), obtemos

$$
\frac{\delta^{2} j_{i}}{\delta A_{l} \delta A_{m}}=i e\left[\tilde{p}_{1, i} \Pi_{l m}^{\mathrm{st}}\left(p_{1}\right)+\tilde{p}_{2, i} \Pi_{l m}^{\mathrm{st}}\left(p_{2}\right)-\tilde{p}_{1, i} \Pi_{l m}^{\mathrm{st}}\left(-p_{1}-p_{2}\right)-\tilde{p}_{2, i} \Pi_{l m}^{\mathrm{st}}\left(-p_{1}-p_{2}\right)\right],
$$

que é exatamente a expressão obtida em (3.34), lembrando da conservação de momento $p_{1}+p_{2}+p_{3}=0$.

Podemos também derivar uma expressão para a ação efetiva estática, que vai envolver integração funcional da corrente, o que parece ser não trivial. De fato isto acontece, mas em um determinado limite podemos derivar uma ação efetiva. Para isto tomamos o limite

$$
\left|p_{a}\right| \ll T,\left|p_{a}\right| \ll|\theta|^{-1 / 2},
$$

onde $a=1,2, \ldots$ indica os índices dos momentos externos. Neste regime o momento interno $k$ deve ser da ordem de $1 /|\tilde{p}|$.

Vamos definir a função $U(p, A)$, dada por

$$
U(p, A)=\int \mathrm{d}^{4} x \exp [-i p \cdot x+i e \tilde{p} \cdot A(x)],
$$

que é uma função do quadrivetor momento $p$ (que pode ser uma combinação de momentos externos $p_{a}$ ), e um funcional com relação às componentes espaciais de $A$. No limite (3.57), a transformação de gauge geral (2.56) pode ser aproximada por

$$
\delta A_{i}(x)=\left[\partial_{i}+i e\left(\tilde{\partial}_{j} A_{i}(x)\right) \partial_{j}\right] \omega(x) .
$$

Por esta transformação, $U$ definido em (3.58) é invariante de gauge. Podemos provar isto da seguinte maneira. Note que

$$
\begin{aligned}
\delta(\tilde{p} \cdot A) & =\left[\tilde{p} \cdot \partial+e\left(\tilde{\partial_{j}} \tilde{p} \cdot A(x)\right) \partial_{j}\right] \omega(x), \\
\delta[\exp (i e \tilde{p} \cdot A(x))] & =i \exp (i e \tilde{p} \cdot A)\left[\tilde{p} \cdot \partial+e\left(\tilde{\partial}_{j} \tilde{p} \cdot A(x)\right) \partial_{j}\right] \omega(x) \\
& =i \exp (i e \tilde{p} \cdot A) \tilde{p} \cdot \partial \omega-\tilde{\partial}_{j}\left[\exp (i e \tilde{p} \cdot A) \partial_{j} \omega\right],
\end{aligned}
$$

onde usamos $\tilde{\partial} \cdot \partial=0$.

Substituindo (3.60) em (3.58) e integrando por partes (de maneira que $\tilde{\partial}_{j}$ age em $e^{-i p \cdot x}$ ), obtemos

$$
\delta U=i \int \mathrm{d}^{4} x e^{-i p \cdot x} \exp (i e \tilde{p} \cdot A)[\tilde{p} \cdot \partial \omega-\tilde{p} \cdot \partial \omega]=0 .
$$

Agora podemos construir a ação efetiva em termos de $U$ :

$$
\Gamma=\frac{1}{2 \times(2 \pi)^{8}} \int \mathrm{d}^{4} p f(\tilde{p}) U(p, A) U(-p, A)=\frac{1}{2 \times(2 \pi)^{8}} \int d^{4} p f(\tilde{p})|U(p, A)|^{2},
$$


onde

$$
f(\tilde{p})=\frac{\tilde{\Pi}_{\mathrm{T}}^{\mathrm{st}}(\tilde{p})}{e^{2} \tilde{p}^{2}}
$$

Esta é a ação efetiva correta no limite (3.57). Ela é invariante de gauge e fornece corretamente as amplitudes de 2, 3 e 4-pontos, como pode-se verificar.

Uma tarefa muito mais difícil é encontrar a ação efetiva no caso quando assumimos apenas o limite $\left|p_{a}\right| \ll T$, ao invés dos dois limites em (3.57). Neste caso temos de usar a transformação de gauge exata (2.55), e não apenas a aproximada (3.59). Mas podemos encontrar uma função generalizada $W$ que seja invariante pela transformação de gauge (2.55), e ela é dada por

$$
W(p, A)=\int \mathrm{d}^{4} x \exp (-i p \cdot x) \star P \exp \left[i e \int_{0}^{1} \mathrm{~d} \xi \tilde{p} \cdot A(x+\xi \tilde{p})\right]
$$

onde $P$ denota um ordenamento de trajetórias na variedade definida pelo produto não-comutativo $\star$. $W(p, A)$ representa a transformada de Fourier de uma linha aberta de Wilson invariante de gauge ${ }^{11}$, que se estende ao longo de uma linha reta que vai de $x$ a $x+\tilde{p}$. Note apenas que se tomarmos o limite (3.57), $W$ se reduz a $U$. Mas mesmo com esta generalização, a ação efetiva não pode ser obtida simplesmente pela substituição de $U$ por $W$ na equação (3.62). A razão é que o momento interno do fóton, $k$, é esperado ser da ordem $1 /\left(\theta\left|p_{a}\right|\right)$, então, sem o limite (3.57), não podemos fazer a aproximação de altas temperaturas $\left|p_{a}\right| \ll|k|$. As amplitudes são, neste caso, muito mais complicadas, e não é evidente que possa ser expressa em termos de uma função simples $f$ do tipo (3.63). Essa é uma questão que precisa ser melhor estudada.

\subsection{Método da amplitude de espalhamento frontal}

Vamos apresentar aqui brevemente outro método de cálculo que já fornece diretamente a parte térmica de um diagrama sem a necessidade de se fazer a soma sobre as freqüências de Matsubara. É o método da amplitude de espalhamento frontal. Como já foi dito, Frenkel, Taylor e Brandt estudaram extensivamente este método. Várias aplicações com este método podem ser encontradas nas referências [46, 47, 48], e sua equivalência com o método tradicional foi demonstrada em [48] para o caso da QCD em um gauge geral covariante. Vamos apresentar aqui os resultados para as amplitudes de 2 e 3-pontos em 1-loop para a parte de gauge da NCQED e também o resultado da amplitude de 2-pontos para o caso de 1-loop fermiônico, todos no limite $p<<k$.

Com este método, o tensor de auto-energia (amplitude de 2-pontos) para a parte de gauge da teoria (diagramas das Figuras 2.1 (a), (b) e (c)) a altas temperaturas pode ser representado simplesmente por

$$
\Pi_{g}^{\mu \nu}(p)=-\left.\frac{4 e^{2}}{(2 \pi)^{3}} \int \frac{\mathrm{d}^{3} \vec{k}}{|\vec{k}|} n_{B}(|\vec{k}|)(1-\cos k \times p)\left[\eta^{\mu \nu}-\frac{p^{\mu} k^{\nu}+p^{\nu} k^{\mu}}{p \cdot k}+\frac{p^{2} k^{\mu} k^{\nu}}{(p \cdot k)^{2}}\right]\right|_{k^{0}=|\vec{k}|}
$$

Os cálculos desta expressão estão feitos no Apêndice D.3, para ilustrar a aplicação do método. Note que

\footnotetext{
${ }^{11}$ Não vamos nos aprofundar neste tema aqui. Para mais referências neste assunto, ver referências [50, 51].
} 
esta é somente a parte dependente da temperatura. Da mesma forma que em (3.9), existe uma parte de vácuo que não estamos considerando aqui, pois queremos analisar apenas a dependência com a temperatura do tensor.

No entanto esta forma do tensor não é muito conveniente para fazermos a integração em $\vec{k}$. Observe que os dois últimos termos proporcionais a $1 /(p \cdot k)$ apresentam uma não-localidade, caracterizada pelo momento externo $p$ no denominador. Por isso vamos reescrever o tensor de maneira mais apropriada fazendo uso da identidade

$$
\frac{p^{\mu} k^{\nu}+p^{\nu} k^{\mu}}{p \cdot k}-\frac{p^{2} k^{\mu} k^{\nu}}{(p \cdot k)^{2}}=p_{\lambda} \frac{\partial}{\partial k_{\lambda}}\left(\frac{k^{\mu} k^{\nu}}{p \cdot k}\right)
$$

Com um pouco de álgebra e fazendo de uso de uma integração por partes pode-se mostrar que a eq. (3.65) pode ser escrita como

$$
\begin{aligned}
\Pi_{g}^{\mu \nu}(p)= & -\frac{4 e^{2}}{(2 \pi)^{3}} \int \frac{\mathrm{d}^{3} \vec{k}}{|\vec{k}|}(1-\cos k \times p)\left[\eta^{\mu \nu} n_{B}(|\vec{k}|)-\left(\eta^{\mu 0} k^{\nu}+\eta^{0 \nu} k^{\mu}\right) \frac{n_{B}(|\vec{k}|)}{|\vec{k}|}\right. \\
& \left.+n_{B}^{\prime}(|\vec{k}|) p_{0} \frac{k^{\mu} k^{\nu}}{\vec{p} \cdot \vec{k}}+\left(\frac{k^{\mu} k^{\nu}}{p_{0} k^{0}-\vec{p} \cdot \vec{k}}\right)\left(\frac{\vec{p} \cdot \vec{k}}{|\vec{k}|} n_{B}^{\prime}(|\vec{k}|)-\frac{\vec{p} \cdot \vec{k}}{|\vec{k}|^{2}} n_{B}(|\vec{k}|)\right)\right]\left.\right|_{k^{0}=|\vec{k}|}
\end{aligned}
$$

onde $n_{B}^{\prime}(|\vec{k}|)$ significa a derivada de $n_{B}$ com relação a $|\vec{k}|$, e então podemos perceber que no limite estático a não-localidade desaparece totalmente, ou seja, fazendo $p_{0}=0$ obtemos finalmente para o tensor

$$
\begin{gathered}
\Pi_{g(\mathrm{st})}^{\mu \nu}(p)=-\frac{4 e^{2}}{(2 \pi)^{3}} \int \frac{\mathrm{d}^{3} \vec{k}}{|\vec{k}|}(1-\cos k \times p)\left[\eta^{\mu \nu} n_{B}(|\vec{k}|)-\frac{k^{\mu} k^{\nu}}{|\vec{k}|} n_{B}^{\prime}(|\vec{k}|)+\frac{k^{\mu} k^{\nu}}{|\vec{k}|^{2}} n_{B}(|\vec{k}|)\right. \\
\left.-\left(\eta^{\mu 0} k^{\nu}+\eta^{0 \nu} k^{\mu}\right) \frac{n_{B}(|\vec{k}|)}{|\vec{k}|}\right]\left.\right|_{k^{0}=|\vec{k}|} \cdot
\end{gathered}
$$

Pode-se ver facilmente que esta expressão dá exatamente a mesma contribuição que (3.9), que foi calculada pelo método direto da soma sobre as freqüências.

Para o caso de 1-loop fermiônico, representado pelo diagrama da Figura 2.1 (d), obtemos a seguinte expressão pelo método da amplitude de espalhamento frontal:

$$
\Pi_{f}^{\mu \nu}(p)=-\left.\frac{8 e^{2}}{(2 \pi)^{3}} \int \frac{\mathrm{d}^{3} \vec{k}}{|\vec{k}|} n_{F}(|\vec{k}|)\left[\eta^{\mu \nu}-\frac{p^{\mu} k^{\nu}+p^{\nu} k^{\mu}}{p \cdot k}+\frac{p^{2} k^{\mu} k^{\nu}}{(p \cdot k)^{2}}\right]\right|_{k^{0}=|\vec{k}|}
$$

Para a amplitude de 3-pontos vinda somente da parte de gauge, representado pelos diagramas da Figura 
3.3, este método fornece

$$
\begin{aligned}
\Gamma_{\mu \nu \lambda}^{\mathrm{g}} & =-\frac{4 i e^{3}}{(2 \pi)^{3}} \sin \left(\frac{p_{1} \times p_{2}}{2}\right) \int \frac{\mathrm{d}^{3} k}{|\vec{k}|} n_{B}(|\vec{k}|)\left\{\left[1-\cos \left(p_{3} \times k\right)\right] L_{\mu \nu \lambda}^{\mathrm{g}}\left(k ; p_{1}, p_{2}, p_{3}\right)\right. \\
& \left.-\left[1-\cos \left(p_{2} \times k\right)\right] L_{\mu \lambda \nu}^{\mathrm{g}}\left(k ; p_{1}, p_{3}, p_{2}\right)-\left[1-\cos \left(p_{1} \times k\right)\right] L_{\lambda \nu \mu}^{\mathrm{g}}\left(k ; p_{3}, p_{2}, p_{1}\right)\right\}\left.\right|_{k_{0}=|\vec{k}|},
\end{aligned}
$$

onde

$$
\begin{aligned}
& L_{\mu \nu \lambda}^{\mathrm{g}}\left(k ; p_{1}, p_{2}, p_{3}\right)=k_{\mu} k_{\nu} \\
& k_{\lambda}\left(\frac{p_{1}^{2}}{\left(k \cdot p_{1}\right)^{2}\left(k \cdot p_{3}\right)}+\frac{p_{1}^{2}}{\left(k \cdot p_{1}\right)^{2}\left(k \cdot p_{2}\right)}-\frac{p_{3}^{2}}{\left(k \cdot p_{3}\right)^{2}\left(k \cdot p_{1}\right)}\right) \\
&+\frac{1}{\left(k \cdot p_{2}\right)\left(k \cdot p_{3}\right)}\left[k_{\nu} k_{\lambda}\left(p_{2}-p_{3}\right)_{\mu}+k_{\mu}\left(k_{\lambda} p_{2 \nu}-k_{\nu} p_{3 \lambda}\right)\right] \\
&+\frac{1}{\left(k \cdot p_{1}\right)\left(k \cdot p_{2}\right)} k_{\mu}\left(k_{\lambda} p_{2_{\nu}}+k_{\nu} p_{2 \lambda}\right)-2 \frac{k_{\mu}}{\left(k \cdot p_{1}\right)} \eta_{\nu \lambda} \\
&-\left(\mu, p_{1}\right) \leftrightarrow\left(\nu, p_{2}\right) .
\end{aligned}
$$

Estas expressões serão importantes no capítulo 5, onde faremos os cálculos usando o método das equações de transporte.

\subsection{Pressão de um gás de fótons não-comutativos}

Antes de terminarmos este capítulo, vamos apresentar brevemente uma aplicação interessante dos métodos da QED não-comutativa para o cálculo da pressão de um gás de fótons não-comutativos. De acordo com o que foi apresentado na seção 1.2.3 do primeiro capítulo, o cálculo da pressão de um gás de fótons (comutativos) a altas temperaturas pode ser feito por meio da função de partição do sistema, ou da energia livre de Helmholtz. Da mesma forma podemos estar interessados nas correções a esta pressão devido a efeitos da não-comutatividade. Isso foi feito primeiramente por Arcioni e Vazquez-Mozo [52], que estudaram as correções de diagramas de 2-loops vindos apenas da parte de gauge da teoria, ou seja, sem considerar contribuições vindas da parte fermiônica. Recentemente, Frenkel, Brandt e Muramoto consideraram as correções vindas de 3-loops no caso da QED sem férmions [53], e depois as contribuições devidas a loops de férmions [54].

Vamos nos restringir a apresentar aqui os resultados do caso de 2-loops da QED sem férmions, que é o que estamos estudando neste capítulo. Os diagramas que contribuem para a energia livre de Helmholtz nesta ordem são dados na Figura 3.5. Pode-se mostrar que as contribuições dos 3 gráficos se reduzem ao cálculo da seguinte expressão para a densidade de energia livre $F$ em segunda ordem ${ }^{12} \mathrm{em} e$,

$$
F^{(2)}(T, \theta)=4 e^{2} \int \frac{\mathrm{d}^{3} q}{(2 \pi)^{3}} \int \frac{\mathrm{d}^{3} p}{(2 \pi)^{3}} \frac{n_{B}(|\vec{q}|)}{|\vec{q}|} \frac{n_{B}(|\vec{p}|)}{|\vec{p}|} \sin ^{2}\left(\frac{p \times q}{2}\right),
$$

\footnotetext{
${ }^{12}$ Não há contribuição de ordem $e$.
} 

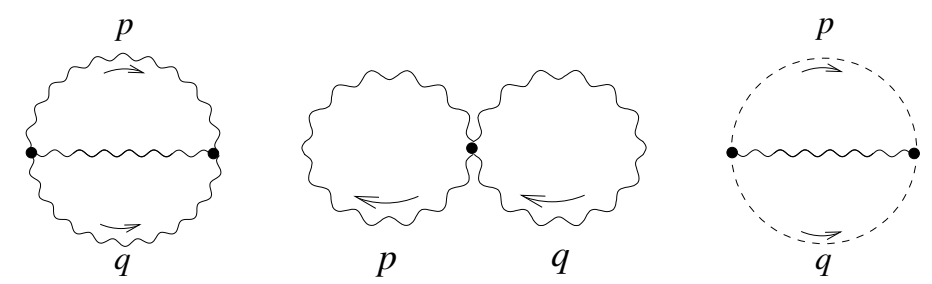

Figura 3.5: Diagramas de 2-loops que contribuem para a energia livre de um gás de fótons não-comutativos.

onde $q$ e $p$ são os momentos internos agora. De acordo com as relações (1.41) e (1.43), a pressão aqui é dada diretamente como o negativo da densidade de energia livre, $P=-F$. No limite de altas temperaturas, quando $\theta T^{2} \gg 1$, a contribuição para a pressão é

$$
P=-F^{(2)}\left(\theta T^{2} \gg 1\right) \approx-\frac{e^{2} T^{4}}{72}+\frac{e^{2} T^{4}}{8 \pi^{2}} \frac{\ln \left(\theta T^{2}\right)}{\theta T^{2}}
$$

indicando surpreendentemente que a altíssimas temperaturas não há dependência com o parâmetro de nãocomutatividade, pois o último termo se anula. Já para o limite $\theta T^{2} \ll 1$ a contribuição é dada por

$$
P=-F^{(2)}\left(\theta T^{2} \ll 1\right) \approx-\frac{e^{2}}{2}\left(\frac{\pi^{2}}{45}\right)^{2} \theta^{2} T^{8}
$$

indicando uma forte dependência $\operatorname{com} \theta$. Para mais discussões neste assunto sugerimos a referência [53].

Neste capítulo apresentamos a primeira parte dos principais resultados de nosso trabalho. O tensor de auto-energia do fóton dependente do parâmetro de não-comutatividade foi encontrado no caso do limite estático a altas temperaturas, que é quando os cálculos se simplificam e podem ser efetuados. Vimos que a temperatura correspondente para que efeitos de não-comutatividade sejam apreciáveis, por exemplo no cálculo de uma seção de choque, deve ser muito alta, assim como a temperatura para que uma massa elétrica se desenvolva no setor não-comutativo. Mostramos que as amplitudes se relacionam por meio de identidades de Ward, de forma que uma ação efetiva que gere amplitudes somente com componentes espaciais pode ser obtida no limite estático. Os principais resultados apresentados neste capítulo, exceto a última seção, foram publicados em [70].

Nos capítulos que se seguem vamos mostrar que os mesmos resultados para as amplitudes podem ser obtidos por um método bem mais direto, que é o método das equações de transporte. 



\section{Capítulo 4}

\section{Equações de Transporte}

Veremos agora uma outra formulação que nos permite fazer cálculos em teoria de campos levando em conta efeitos de temperatura de um sistema. É o método das equações de transporte. Vamos mostrar que muitos dos resultados obtidos de maneira trabalhosa na formulação tradicional da teoria de campos podem ser obtidos, pelo menos em alguns limites, por este método muito mais intuitivo, já que a temperatura entra de forma natural nos cálculos. Toda a construção da teoria das equações de transporte é baseada na teoria cinética dos gases, formulada por Boltzmann e outros já na segunda metade do século 19. A idéia principal é de que as propriedades de interesse de um sistema qualquer em equilíbrio podem ser representadas em termos de uma função, chamada função distribuição. Quando o sistema sofre alguma interação, podemos caracterizar as mudanças ocorridas no sistema por uma mudança na função distribuição, que deixa de ser a de equilíbrio. A equação que deve ser satisfeita pela função distribuição é a equação de transporte, que pode ser obtida basicamente conhecendo-se o tipo da interação que age no sistema, ou seja, suas equações dinâmicas. A equação de transporte pode ser resolvida iterativamente, de forma que correções à função distribuição podem ser obtidas, e desta forma as propriedades de interesse do sistema podem ser obtidas ordem a ordem, como correções às propriedades do sistema em equilíbrio.

O primeiro passo no sentido de se estender a teoria cinética dos gases - conforme desenvolvida por Bernoulli, Clausius, Maxwell e Boltzmann - para o domínio relativístico, foi feito por Jüttner em 1911. Ele derivou uma generalização relativística para a função distribuição de Maxwell, e em 1928 ele estabeleceu a forma da função distribuição em equilíbrio válida para sistemas de bósons e férmions. O passo seguinte para o desenvolvimento de uma equação cinética relativística, que é a equação que a função distribuição deve satisfazer, foi dado por Walker em 1935 para o caso de partículas que não sofram colisões entre si. Foi somente em 1946 que uma generalização relativística da equação de Boltzmann, incluindo efeitos de colisão, foi publicada por Marrot, e seguiram-se estudos de Chernikov, Clemmow, Willson e Bergmann. Ao mesmo tempo outros tipos de equações cinéticas relativísticas foram consideradas, em particular a generalização relativística da equação de Vlasov e da equação de Fokker-Planck.

Um dos propósitos da teoria cinética é derivar leis macroscópicas de conservação baseada na equação cinética adotada. Marrot e Taub foram os primeiros a mostrar que leis macroscópicas de massa, momento e energia podiam ser obtidas desta maneira. A generalização relativística do teorema $H$ da termodinâmica 
- que garante a positividade da entropia para sistemas fora do equilíbrio - foi derivada por Marrot, Ehlers, Tauber, Weinberg e Chernikov. Muitas das fórmulas obtidas nesta época para um sistema gasoso relativístico em equilíbrio ainda estavam em uma forma que não eram manifestamente covariante, e uma vez que estas equações fossem escritas covariantemente, elas poderiam ser aplicadas para a gravitação. Isto foi feito primeiramente por Marrot, Weinberg e Chernikov, que colocaram as equações no contexto da relatividade geral. Formalmente, o que eles fizeram foi considerar derivadas covariantes em lugar das derivadas ordinárias do espaço-tempo. Isto resultou numa teoria em que a gravidade aparece como uma força de longo alcance auto-consistente. Como esta força não influencia as propriedades locais do sistema, que sofrem ação somente de forças de curto alcance, a estrutura formal da teoria permanece essencialmente a mesma. No início da década de 60, Israel, Kelly e Chernikov adaptaram, independentemente, os métodos clássicos de Chapman-Enskog e de Maxwell e Grad ao domínio relativístico, e por muitos anos vários pesquisadores trabalharam e desenvolveram estes métodos. Desta maneira, cada vez mais tornou-se mais fácil determinar expressões para os coeficientes de transporte de sistemas de partículas movendo-se a velocidades relativísticas, e mesmo para sistemas contendo neutrinos ou fótons. Na década de 70 Weinberg estudou a formação de galáxias com estes modelos, o que serviu como uma boa motivação para a construção de uma descrição coerente de fenômenos fora do equilíbrio ${ }^{1}$.

Como já foi dito na introdução, existem duas maneiras de se obter a função distribuição de um sistema por meio das equações de transporte. A primeira é a forma clássica, que se baseia nas equações de movimento clássicas do sistema. A segunda é uma formulação quântica, desenvolvida por Wigner. Vamos apresentar as duas formulações aqui. Nosso objetivo é estudar as amplitudes de $n$-pontos em 1-loop da eletrodinâmica quântica não-comutativa por meio das equações de transporte, que será feito no próximo capítulo. Neste capítulo vamos apenas apresentar a teoria.

\subsection{Teoria clássica de transporte}

As propriedades de um sistema de muitos corpos depende essencialmente das interações entre as partículas que constituem o sistema e das interações devido a ação de alguma força externa. Estaremos interessados em obter certas propriedades macroscópicas deste sistema, como pressão, energia, entropia, etc, no equilíbrio. Nosso propósito então é expressar estas quantidades macroscópicas (que são funções do espaço-tempo) em termos de variáveis de estado macroscópicas, como densidade de partícula e temperatura por exemplo, e de parâmetros microscópicos característicos do sistema. Na teoria cinética dos gases isto é feito por meio de descrições estatísticas em termos da chamada função distribuição de uma partícula. Esta função pode ser interpretada como sendo a função que fornece o número médio de partículas com um certo momento $\vec{p}$ em cada ponto do espaço-tempo $(\vec{x}, t)$. Para encontrar a forma explícita desta função, temos de postular ou derivar equações cinéticas satisfeitas pelas partículas, equações estas também chamadas de equações de transporte. Estas equações estabelecem como a função distribuição muda no espaço e no tempo devido às interações das partículas. Na prática estas equações são difíceis de serem resolvidas

\footnotetext{
${ }^{1}$ Uma boa lista de referências nestes assuntos estão na introdução de [56].
} 
exatamente e temos de nos limitar a simplificações, como por exemplo considerar apenas colisões entre duas partículas e considerar sistemas perto do equilíbrio, mas mesmo nestes casos obtemos bons resultados. Vamos então introduzir algumas quantidades físicas de interesse, onde estaremos trabalhando com a notação relativística usual $x=x^{\mu}=\left(x^{0}, \vec{x}\right)$ e $p=p^{\mu}=\left(p^{0}, \vec{p}\right)$ onde $x^{0}=c t$ e $c p^{0}=\sqrt{\vec{p}^{2} c^{2}+m^{2} c^{4}}$ é a energia relativística de uma partícula de massa $m$ e $c$ é a velocidade da luz.

\subsubsection{Corrente de partículas}

Para descrever um sistema não-uniforme, introduzimos uma densidade local $n(\vec{x}, t)$, de maneira que $n(\vec{x}, t) \Delta^{3} x$ nos dá o número médio de partículas no elemento de volume $\Delta^{3} x$ localizado no ponto $\vec{x}$ no instante de tempo $t$. Da mesma maneira definimos o fluxo de partículas $\vec{j}(\vec{x}, t)$, assim formamos o quadrivetor corrente ou simplesmente corrente de partículas, dado por:

$$
J^{\mu}(x)=(\operatorname{cn}(\vec{x}, t), \vec{j}(\vec{x}, t)) .
$$

Se o número de partículas for grande, então faz sentido introduzirmos uma função $f(x, p)$ que dê a distribuição do momento $p$ em cada ponto do espaço-tempo. Esta definição é tal que $f(x, p) \Delta^{3} x \Delta^{3} p$ dá o número médio de partículas que no instante $t$ estejam localizadas no elemento de volume $\Delta^{3} x$ em torno de $\vec{x}$ e com momento entre $\vec{p}$ e $\vec{p}+\Delta \vec{p}$. Esta definição pressupõe que o número de partículas contida no volume $\Delta^{3} x$ seja grande mas por outro lado que o tamanho $\Delta^{3} x$ é pequeno do ponto de vista macroscópico. Em termos da função distribuição a densidade e o fluxo de partículas podem ser escritos como

$$
\begin{aligned}
& n(\vec{x}, t)=\int \mathrm{d}^{3} p f(x, p), \\
& \vec{j}(\vec{x}, t)=\int \mathrm{d}^{3} p \vec{u} f(x, p),
\end{aligned}
$$

onde

$$
\vec{u}=\frac{c \vec{p}}{p^{0}}
$$

é a velocidade de uma partícula relativística com momento $\vec{p}$. Como conseqüência de (4.2) e (4.3) podemos escrever o quadrivetor corrente (4.1) como

$$
J^{\mu}(x)=c \int \frac{\mathrm{d}^{3} p}{p^{0}} p^{\mu} f(x, p) .
$$

Esta expressão mostra que a função distribuição $f(x, p)$ deve ser um escalar por transformações de Lorentz. Usando a identidade

$$
\theta\left(p^{0}\right) \delta\left(p^{2}-m^{2} c^{2}\right)=\frac{1}{2 p^{0}} \delta\left(p^{0}-\sqrt{\vec{p}^{2}+m^{2} c^{2}}\right),
$$


onde $\theta$ é a função degrau unitário, $\delta$ é a função delta de Dirac e $p^{2}=p^{\mu} p_{\mu}=\left(p^{0}\right)^{2}-\vec{p}^{2}=m^{2} c^{2}$, podemos reescrever a corrente (5.88) como

$$
J^{\mu}(x)=2 c \int \mathrm{d}^{4} p \theta\left(p^{0}\right) \delta\left(p^{2}-m^{2} c^{2}\right) p^{\mu} f(x, p) .
$$

Assumimos aqui, sem uma demonstração rigorosa, que $J^{\mu}(x)$ se transforma como um quadrivetor de Lorentz, assim como o fato de a função distribuição $f(x, p)$ ser um escalar ${ }^{2}$.

\subsubsection{Tensor de energia-momento}

Vamos considerar agora a densidade de energia. Desde que a energia por partícula é $c p^{0}$, podemos escrever a densidade de energia macroscópica $T^{00}$ como sendo:

$$
T^{00}(x)=c \int \mathrm{d}^{3} p p^{0} f(x, p)
$$

De maneira semelhante definimos o fluxo de energia $c T^{0 j}$ como

$$
c T^{0 j}(x)=c \int \mathrm{d}^{3} p p^{0} u^{j} f(x, p), \quad j=1,2,3
$$

e a densidade de momento $T^{i 0} / c$, que é o valor médio do momento $\vec{p}$ da partícula, como sendo

$$
T^{i 0}(x) / c=\int \mathrm{d}^{3} p p^{i} f(x, p) . \quad i=1,2,3
$$

Finalmente, definimos o fluxo de momento $T^{i j}$ (ou tensor de pressão) que é o fluxo na direção $j$ do momento na direção $i$

$$
T^{i j}(x)=\int \mathrm{d}^{3} p p^{i} u^{j} f(x, p) .
$$

Estas quantidades podem ser expressas por meio de um tensor, chamado tensor de energia-momento, que é dado pela forma covariante

$$
T^{\mu \nu}(x)=2 c \int \mathrm{d}^{4} p \theta\left(p_{0}\right) \delta\left(p^{2}-m^{2} c^{2}\right) p^{\mu} p^{\nu} f(x, p) .
$$

É importante notar aqui que esta definição do tensor momento-energia leva em conta apenas a energia de repouso das partículas e suas energias cinéticas. Estamos assumindo que as possíveis energias de interação entre as partículas são pequenas comparadas com suas energias cinéticas. Se não fosse assim, o tensor energia-momento deveria conter também uma contribuição devido a energia potencial.

Veremos agora como obter a função distribuição do sistema, ou melhor, que equação deve ser satisfeita por $f$ para que possamos determiná-lo. É a equação de transporte.

\footnotetext{
${ }^{2}$ Mais detalhes sobre esta discussão estão na referência [56].
} 


\subsubsection{Equação de transporte para $f(\vec{x}, \vec{p}, t)$}

O principal objeto de estudo na teoria de transporte é a chamada função distribuição $f(\vec{x}, \vec{p}, t)$, que representa a densidade de partículas com momento $\vec{p}$ em um ponto $\vec{x}$ do espaço num instante de tempo $t$. Na mecânica estatística isto é conhecido como densidade do espaço de fases. Por exemplo, para uma única partícula no ponto $x_{1}$ com momento $p_{1}$, no espaço unidimensional teríamos $f(x, p, t)=\delta\left(x-x_{1}(t)\right) \delta(p-$ $\left.p_{1}(t)\right)$. Para um sistema com $N$ partículas, um gás ou um plasma por exemplo, a generalização é imediata

$$
f(\vec{x}, \vec{p}, t)=\sum_{i=1}^{N} \delta\left(\vec{x}-\vec{x}_{i}(t)\right) \delta\left(\vec{p}-\vec{p}_{i}(t)\right)
$$

sendo $\vec{x}_{i}(t)$ e $\vec{p}_{i}(t)$ os vetores posição e momento da $i$-ésima partícula do sistema no instante $t$. As equações de movimento satisfeitas por $\vec{x}_{i}(t)$ e $\vec{p}_{i}(t)$ são

$$
\begin{aligned}
\dot{\vec{x}}_{i} & =\frac{\vec{p}_{i}}{m} \\
\dot{\vec{p}}_{i} & =\vec{F}\left(\vec{x}_{i}(t)\right)
\end{aligned}
$$

sendo $\vec{F}\left(\vec{x}_{i}(t)\right)$ a soma de todas as forças que agem na partícula localizada em $\vec{x}_{i}$. Se, por simplicidade, pensarmos que este nosso sistema é composto por moléculas de um gás e que as colisões entre elas são desprezíveis, temos então que cada molécula forma um subsistema independente, assim a função distribuição das moléculas deve obedecer ao teorema de Liouville, que nos diz que ela deve ser constante no espaço de fases, caracterizando um estado de equilíbrio do sistema,

$$
\frac{\mathrm{d}}{\mathrm{d} t} f(\vec{x}, \vec{p}, t)=0
$$

sendo a derivada total correspondente a uma diferenciação ao longo do espaço de fases da molécula, determinado pelas equações do movimento (4.14). Na ausência de uma força externa temos

$$
\frac{\mathrm{d}}{\mathrm{d} t} f=\frac{\partial}{\partial t} f+\vec{v} \cdot \vec{\nabla} f
$$

Se, por outro lado, a molécula estiver sob a influência de uma força externa $\vec{F}$, então

$$
\frac{\mathrm{d}}{\mathrm{d} t} f=\frac{\partial}{\partial t} f+\frac{\vec{p}}{m} \cdot \vec{\nabla} f+\vec{F} \cdot \frac{\partial f}{\partial \vec{p}} .
$$

onde usamos $\vec{v}=\vec{p} / m$. Se a colisão entre as moléculas for levada em conta, então a equação (4.15) não é mais válida, e a função distribuição não é mais constante ao longo do espaço de fases. Devemos ter então

$$
\frac{\mathrm{d}}{\mathrm{d} t} f=C(f)
$$

onde $C(f)$ é chamado integral de colisão e caracteriza a taxa de mudança da função distribuição devido às colisões. Obtemos então a chamada equação de transporte para uma partícula clássica sujeita às equações 
de movimento (4.14),

$$
\left(\frac{\partial}{\partial t}+\frac{\vec{p}}{m} \cdot \vec{\nabla}+\vec{F} \cdot \frac{\partial}{\partial \vec{p}}\right) f=C(f) .
$$

Em geral é bastante complicado encontrar a integral de colisão $C(f)$, e em muitas aplicações a primeira aproximação é tomá-la igual a zero. Desta forma, conhecida a forma da força que age na partícula, por mais complicada que ela seja, podemos resolver a equação acima iterativamente e obter a expressão para $f$ ordem a ordem. Isto fica mais claro se tomarmos o caso da força eletromagnética. Em notação relativística, tomando $c=1$ por simplicidade, as coordenadas do espaço-tempo serão representadas por $x^{\mu}=(t, \vec{x}) \mathrm{e}$ o momento $p^{\mu}=\left(p^{0}, \vec{p}\right)$, com $p^{0}=\sqrt{\vec{p}^{2}+m^{2}}$. O vetor velocidade é dado por $\vec{v}=\vec{p} / p^{0}$, e o operador gradiente $\partial_{\mu} \equiv \partial / \partial x^{\mu}=\left(\partial_{t}, \vec{\nabla}\right)$. Desta forma, tomando $C(f)=0$, a equação (4.19) pode ser escrita como

$$
p^{\mu} \partial_{\mu} f(x, p)=-e p_{\nu} F^{\mu \nu} \frac{\partial f}{\partial p^{\mu}}
$$

onde $F^{\mu \nu}=\partial^{\mu} A^{\nu}-\partial_{\nu} A^{\mu}$ é o tensor eletromagnético. Esta é a equação de Vlasov, que pode ser resolvida da seguinte maneira. Veja que o lado esquerdo é de ordem zero no acoplamento $e$, enquanto que o lado direito é de primeira ordem. Portanto, expandindo $f$ em potências de $e$,

$$
f=f^{(0)}+e f^{(1)}+e^{2} f^{(2)}+\cdots
$$

e conhecendo-se o termo de ordem zero, $f^{(0)}$, que é simplesmente a solução do problema sem interação, obtém-se, com a equação (4.20), o termo $f^{(1)}$. Repetindo o processo obtém-se o termo $f^{(2)}$, e assim por diante, de forma que todas as quantidades físicas de interesse, por exemplo a corrente (4.7) e o tensor de energia-momento (4.12), podem ser obtidos com precisão cada vez maior, desde que as correções à função distribuição do sistema sejam obtidas. Esta é a idéia básica por trás do método das equações de transporte.

\subsection{Teoria quântica de transporte}

Nesta seção vamos apresentar outro método de se trabalhar com as equações de transporte, que é a formulação quântica inicialmente desenvolvida por Wigner [57], e posteriormente estudada por vários outros autores em diferentes contextos. Vale citar em especial os trabalhos de Elze, Heinz, Vasak e Gyulassy $[58,59,60]$, entre outros, que deram uma atenção especial a este assunto com o objetivo de aplicar os métodos cinéticos existentes na época para estudar sistemas de quarks e glúons da QCD. O problema maior era que, como as interações entre quarks e glúons devem ser descritas por uma teoria de gauge nãoabeliana, a teoria cinética correspondente deveria fornecer resultados invariantes de gauge, e portanto ser formulada de uma forma covariante de gauge desde o início. Para isto era necessária uma modificação na definição da função distribuição para o espaço de fases com relação às definições existentes. Além disso, uma função distribuição quântica para campos de gauge ainda não existia, até o advento dos estudos em física de plasmas de quarks e glúons, e era necessária uma formulação covariante de gauge para tais 
sistemas.

A breve discussão que se segue é baseada nos trabalhos daqueles autores para uma formulação consistente da teoria de transporte para quarks e glúons, conforme [60].

\subsubsection{Operador de Wigner não-relativístico}

Vamos ver agora qual o equivalente da função distribuição clássica $f(\vec{x}, \vec{p}, t)$ no caso da mecânica quântica. Sabemos que, para uma função de onda $\psi(x)$ que seja solução da equação de Schrödinger

$$
\left[-i \hbar \frac{\partial}{\partial t}-\frac{\hbar^{2}}{2 m} \nabla^{2}+V(x)\right] \psi(x)=0
$$

a quantidade $\psi^{\dagger}(x) \psi(x)$ representa a densidade de partículas no espaço das coordenadas, onde $x$ agora diz respeito tanto ao trivetor $\vec{x}$ quanto ao tempo $t$. Para o momento $p$ devemos levar em consideração que ele age como um operador $\hat{p}$ no espaço das coordenadas. Assim, em analogia com a equação (4.13) do caso clássico, obtemos o análogo quântico da densidade no espaço de fases, a chamada função de Wigner [57]

$$
W(x, p)=\psi^{\dagger}(x) \delta(p-\hat{p}) \psi(x)
$$

A função delta age como um projetor no espaço dos momentos e é melhor definida como uma transformada de Fourier. Desta maneira a equação (4.23) fica

$$
W(x, p)=\int \frac{\mathrm{d}^{3} y}{(2 \pi \hbar)^{3}} \psi^{\dagger}(x) \mathrm{e}^{i y(p-\hat{p}) / \hbar} \psi(x) .
$$

A fim de que $W$ seja hermiteano, faremos com que o operador $\hat{p}$ aja tanto em $\psi$ quanto em $\psi^{\dagger}$, substituindo $\hat{p} \rightarrow-\frac{1}{2} i \hbar\left(\overrightarrow{\partial_{x}}-\overleftarrow{\partial_{x}}\right)$, de maneira que obtemos

$$
W(x, p)=\int \frac{\mathrm{d}^{3} y}{(2 \pi \hbar)^{3}} \mathrm{e}^{i y p / \hbar} \psi^{\dagger}(x) \mathrm{e}^{-\frac{1}{2} y\left(\overrightarrow{\partial_{x}}-\overleftarrow{\partial_{x}}\right)} \psi(x)
$$

Para finalizar, observe que o operador $\exp \left(-y \partial_{x}\right)$ gera uma translação por uma quantidade $-y$ quando agindo à direita,

$$
\mathrm{e}^{-y \partial_{x}} f(x)=f(x-y)
$$

que pode ser facilmente demonstrado expandindo-se a exponencial e tomando a expansão de Taylor de uma função. Desta maneira obtemos uma expressão mais familiar para a função de Wigner,

$$
W(x, p)=\int \frac{\mathrm{d}^{3} y}{(2 \pi \hbar)^{3}} \mathrm{e}^{i y p / \hbar} \psi^{\dagger}\left(x+\frac{1}{2} y\right) \psi\left(x-\frac{1}{2} y\right) .
$$

A equação de transporte a ser satisfeita por $W$ deve seguir da equação de Schrödinger (4.22). Vamos 
começar calculando a quantidade $\vec{p} \cdot \vec{\nabla} W$. Ela é dada por

$$
\begin{aligned}
\vec{p} \cdot \vec{\nabla} W(x, p) & =m \int \frac{\mathrm{d}^{3} y}{(2 \pi \hbar)^{3}} \mathrm{e}^{i y p / \hbar}\left(-\partial_{t}+\frac{i}{\hbar}\left[V\left(x+\frac{y}{2}\right)-V\left(x-\frac{y}{2}\right)\right]\right) \psi^{\dagger}\left(x+\frac{y}{2}\right) \psi\left(x-\frac{y}{2}\right) \\
& =m \int \frac{\mathrm{d}^{3} y}{(2 \pi \hbar)^{3}} \mathrm{e}^{i y p / \hbar}\left(-\partial_{t}+\frac{i}{\hbar}\left(\mathrm{e}^{-y \nabla_{x} / 2}-\mathrm{e}^{y \nabla_{x} / 2}\right) V(x)\right) \psi^{\dagger}\left(x+\frac{y}{2}\right) \psi\left(x-\frac{y}{2}\right) \\
& =m\left(-\partial_{t}+\frac{2}{h} \sin \left(\frac{\hbar}{2} \vec{\nabla}_{x} \cdot \vec{\nabla}_{p}\right) V(x)\right) W(x, p)
\end{aligned}
$$

onde $\vec{\nabla}_{x}$ age somente em $V(x)$ e $\vec{\nabla}_{p}$ age em $W$. Para obter esta expressão note que, dentro da integral, fizemos $\vec{p} \rightarrow i \hbar \vec{\nabla}_{y}$ após uma integração por partes. Depois escrevemos

$$
\vec{\nabla}_{y} \cdot \vec{\nabla}_{x}=\frac{1}{2}\left(\nabla_{x+y / 2}^{2}-\nabla_{x-y / 2}^{2}\right)
$$

e usamos a equação de Schrödinger (4.22) para substituir $\nabla^{2}$. Na segunda linha usamos o operador de translação (4.25), e para obter a última linha trocamos $y \rightarrow-i \hbar \nabla_{p}$, de forma que a exponencial pode ser colocada fora da integração. Desta forma, obtemos a equação de transporte não-relativística para $W$,

$$
\begin{aligned}
\left(m \partial_{t}+\vec{p} \cdot \vec{\nabla}_{x}-\vec{\nabla}_{x} V \cdot \vec{\nabla}_{p}\right) W(x, p) & =\left(\frac{2}{\hbar} \sin \left(\frac{\hbar}{2} \vec{\nabla}_{x} \cdot \vec{\nabla}_{p}\right)-\vec{\nabla}_{x} \cdot \vec{\nabla}_{p}\right) V(x) W(x, p) \\
& \simeq-\frac{1}{24} \hbar^{2}\left(\vec{\nabla}_{x} \cdot \vec{\nabla}_{p}\right)^{3} V(x) W(x, p)
\end{aligned}
$$

onde usamos a expansão da função seno (C.9) na última linha para ficar mais claro o resultado. Em primeiro lugar note que esta expressão é exatamente análoga a (4.19). No limite $\hbar \rightarrow 0$ obtemos a equação clássica de Vlasov com $\vec{F}=-\vec{\nabla} V$. Esta é, portanto, a equação de transporte que fornece as correções quânticas (proporcionais a $\hbar$ ) à função distribuição de Wigner. O lado esquerdo é independente de $\hbar$, enquanto que o lado direito depende do "acoplamento" quântico $\hbar$. Podemos então obter ordem a ordem as correções a $W$, e com isto calcular correções quânticas a sistemas clássicos.

\subsubsection{Operador de Wigner relativístico}

Para trabalharmos com a teoria de campos relativística e sermos capazes de passar de uma descrição de uma única partícula para uma teoria de muitos corpos, precisamos fazer a segunda quantização na função de Wigner e escrevê-la como um operador $\hat{W}$. Para isto basta trocar $\psi(x)$ pelo operador de campo de Heisenberg $\hat{\psi}(x)$. Uma média apropriada de $\hat{W}$ irá corresponder à densidade no espaço de fases clássico. O operador de Wigner relativístico $\hat{W}$ é definido como

$$
\hat{W}(x, p)=\int \frac{\mathrm{d}^{4} y}{(2 \pi \hbar)^{4}} \mathrm{e}^{i y p / \hbar} \hat{\psi}^{\dagger}\left(x+\frac{1}{2} y\right) \hat{\psi}\left(x-\frac{1}{2} y\right),
$$


e a equação de transporte deve ser obtida usando-se as equações satisfeitas pelos campos $\hat{\psi}(x)$, e isto vai depender de a partícula ser um bóson ou um férmion.

\subsubsection{Operador de Wigner para férmions}

Vamos brevemente apresentar aqui o operador de Wigner para o caso de partículas de spin $\frac{1}{2}$, quarks por exemplo, representadas por um espinor de quatro componentes $\psi(x)$, que satisfaz a equação de Dirac

$$
\left(i \hbar \gamma^{\mu} D_{\mu}(x)-m c\right) \hat{\psi}(x)=0
$$

$D_{\mu}$ é a derivada covariante, quando agindo em um operador de campo fermiônico tem a forma

$$
D_{\mu}(x)=\frac{\partial}{\partial x^{\mu}}-\frac{i g}{\hbar c} A_{\mu}(x),
$$

e quando agindo em um operador de campo de gauge tem a forma

$$
D_{\mu}(x)=\frac{\partial}{\partial x^{\mu}}-\frac{i g}{\hbar c}\left[A_{\mu}(x),\right]
$$

Queremos discutir a covariância de gauge do operador de Wigner. É imediato supormos que o operador tenha a forma

$$
\begin{aligned}
\hat{W}(x, p) & =\int \frac{\mathrm{d}^{4} y}{(2 \pi \hbar)^{4}} \mathrm{e}^{i y p / \hbar} \hat{\bar{\psi}}\left(x+\frac{1}{2} y\right) \otimes \hat{\psi}\left(x-\frac{1}{2} y\right) \\
& =\int \frac{\mathrm{d}^{4} y}{(2 \pi \hbar)^{4}} \mathrm{e}^{i y p / \hbar} \mathrm{e}^{\frac{1}{2} y \cdot \partial_{x}} \hat{\bar{\psi}}(x) \otimes \mathrm{e}^{-\frac{1}{2} y \cdot \partial_{x}} \hat{\psi}(x)
\end{aligned}
$$

onde o produto tensorial $\otimes$ implica que $\hat{W}$ é uma matriz $4 \times 4$ nos índices espinoriais e $\bar{\psi}=\psi^{\dagger} \gamma^{0}$. Mas esta definição ainda não está correta, pelo menos para uma teoria de gauge sabemos que ela não se transforma covariantemente. Podemos encontrar uma definição que seja covariante por transformações de gauge se fizermos a substituição da derivada usual pela derivada covariante (4.32), de maneira que obtemos o operador de Wigner para partículas de spin $\frac{1}{2}$,

$$
\hat{W}(x, p)=\int \frac{\mathrm{d}^{4} y}{(2 \pi \hbar)^{4}} \mathrm{e}^{i y p / \hbar} \mathrm{e}^{\frac{1}{2} y \cdot D^{\dagger}(x)} \hat{\bar{\psi}}(x) \otimes \mathrm{e}^{-\frac{1}{2} y \cdot D(x)} \hat{\psi}(x) .
$$

Por uma transformação de gauge local, $S(x)=\exp i \theta_{a}(x) t_{a}$, temos $\hat{\psi}(x) \rightarrow S(x) \hat{\psi}(x)$ e $D^{\mu} \rightarrow$ $S D^{\mu} S^{-1}$, e o operador de Wigner se transforma covariantemente

$$
\hat{W}(x, p) \rightarrow S(x) \hat{W}(x, p) S^{-1}(x) .
$$

O procedimento para se obter a equação de transporte correspondente é o mesmo adotado no exemplo da equação de Schrödinger. Calcula-se a quantidade $p^{\mu} D_{\mu}(x) \hat{W}(x, p)$, que é o análogo relativístico quadridimensional de $\vec{p} \cdot \vec{\nabla} W$, e em algum momento usa-se a equação de Dirac (4.31). Os detalhes são 
complicados e o cálculo exato bastante trabalhoso. Vamos apenas apresentar o resultado para a ordem dominante, a fim de ilustrar a forma da equação de transporte. Para o leitor interessado sugerimos a referência [58], onde a equação completa está feita em detalhes, inclusive com a adição de efeitos de spin. A expressão que se obtém para ordem dominante da equação de transporte para $\hat{W}$ no caso de férmions é

$$
\begin{aligned}
p^{\mu} D_{\mu}(x) \hat{W}(x, p)=-\frac{g}{2 c} p^{\mu} \partial_{p}^{\nu} \int_{0}^{1} \mathrm{~d} s\left\{\left[\mathrm{e}^{-s \Delta} F_{\mu \nu}(x)\right] \hat{W}(x, p)+\hat{W}(x, p)\left[\mathrm{e}^{s \Delta} F_{\mu \nu}(x)\right]\right\} \\
+\frac{i g}{4 c} \hbar \partial_{p}^{\nu} \int_{0}^{1} \mathrm{~d} s s\left\{\left[\mathrm{e}^{-s \Delta} F_{\mu \nu}(x)\right] D^{\mu}(x) \hat{W}(x, p)-D^{\mu}(x) \hat{W}(x, p)\left[\mathrm{e}^{s \Delta} F_{\mu \nu}(x)\right]\right\},
\end{aligned}
$$

onde $\Delta \equiv \frac{1}{2} i \hbar \partial_{p} \cdot D(x)$.

\subsubsection{Operador de Wigner para campos de gauge}

Para campos de gauge que se transformam covariantemente pelo grupo de gauge $\mathrm{SU}(\mathrm{N})$, o tensor de campo que representa a partícula é dado por $F_{\mu \nu}=F_{\mu \nu}^{a} t_{a}$, onde

$$
F_{\mu \nu}^{a}=\partial_{\mu} A_{\nu}^{a}-\partial_{\nu} A_{\mu}^{a}+\frac{g}{c} f_{a b c} A_{\mu}^{b} A_{\nu}^{c},
$$

e $t_{a}$ são os geradores do grupo $\mathrm{SU}(\mathrm{N})$ que satisfazem

$$
\left[t_{a}, t_{b}\right]=i \hbar f_{a b c} t_{c}
$$

e a equação de movimento para o campo é

$$
D_{\mu}(x) F^{\mu \nu}(x)=\left[D_{\mu}(x), F^{\mu \nu}(x)\right]=-\frac{g}{c} j^{\nu}(x) .
$$

Em analogia com o operador de Wigner definido para férmions anteriormente, o correspondente operador de Wigner para campos de gauge não-abelianos (glúons por exemplo) em termos dos operadores de campo $F^{\mu \nu}(x)$ é

$$
\hat{\Gamma}_{\mu \nu}(x, p)=\int \frac{\mathrm{d}^{4} y}{(2 \pi \hbar)^{4}} \mathrm{e}^{-i y p / \hbar}\left[\mathrm{e}^{\frac{1}{2} y \cdot D(x)} F_{\mu}^{\lambda}(x)\right] \otimes\left[\mathrm{e}^{-\frac{1}{2} y \cdot D(x)} F_{\lambda \nu}(x)\right]
$$

e o produto tensorial aqui significa que $\hat{\Gamma}$ é uma matriz $N \times N$ nos índices de cor. Usando o análogo covariante do operador de translação (4.25) agindo em um operador $O(x)$,

$$
\mathrm{e}^{-y \cdot D(x)} O(x)=U(x, x-y) O(x-y) U(x-y, x),
$$


obtemos uma forma mais conveniente para o operador de Wigner (4.40),

$$
\hat{\Gamma}_{\mu \nu}(x, p)=\int \frac{\mathrm{d}^{4} y}{(2 \pi \hbar)^{4}} \mathrm{e}^{-i y p / \hbar} U\left(x, x_{2}\right) F_{\mu}^{\lambda}\left(x_{2}\right) U\left(x_{2}, x\right) \otimes U\left(x, x_{1}\right) F_{\lambda \nu}\left(x_{1}\right) U\left(x_{1}, x\right),
$$

onde $x_{1}=x-\frac{y}{2}, x_{2}=x+\frac{y}{2}$ e o operador

$$
U(a, b)=P \exp \left(\frac{i g}{\hbar c} \int_{b}^{a} \mathrm{~d} z^{\mu} A_{\mu}(z)\right)
$$

é o chamado "operador de link". Aqui, $P$ indica um ordenamento do caminho de integração entre os pontos $a$ e $b$, que deve ser tomado com sendo uma linha reta

$$
z(s)=a+s(b-a), \quad 0 \leq s \leq 1
$$

para que a propriedade (4.41) seja satisfeita.

A equação de transporte satisfeita pela função distribuição de Wigner para este caso é, em ordem dominante

$$
\begin{aligned}
p \cdot D(x) \hat{\Gamma}_{\mu \nu}(x, p)= & -\frac{g}{2 c} p^{\sigma} \partial_{p}^{\tau} \int_{0}^{1} \mathrm{~d} s\left[\left(\mathrm{e}^{-s \Delta} \mathcal{F}_{\sigma \tau}\right) \hat{\Gamma}_{\mu \nu}+\hat{\Gamma}_{\mu \nu}\left(\mathrm{e}^{s \Delta} \mathcal{F}_{\sigma \tau}\right)\right] \\
& +\frac{i g}{4 c} \hbar \partial_{p}^{\tau} \int_{0}^{1} \mathrm{~d} s s\left[\left(\mathrm{e}^{-s \Delta} \mathcal{F}_{\tau \sigma}\right) D^{\sigma} \hat{\Gamma}_{\mu \nu}-D^{\sigma} \hat{\Gamma}_{\mu \nu}\left(\mathrm{e}^{s \Delta} \mathcal{F}_{\tau \sigma}\right)\right] \\
& -\frac{g}{c}\left[\left(\mathrm{e}^{-\Delta} \mathcal{F}_{\mu \lambda}\right) \hat{\Gamma}_{\nu}^{\lambda}-\hat{\Gamma}_{\mu}^{\lambda}\left(\mathrm{e}^{\Delta} \mathcal{F}_{\lambda \nu}\right)\right]
\end{aligned}
$$

onde $\Delta \equiv \frac{1}{2} i \hbar \partial_{p} \cdot D(x)$. Os detalhes desta dedução podem ser encontrados em [59].

A conexão com observáveis físicos do campo de gauge pode ser feito por meio da definição do tensor de energia-momento em termos da função de Wigner, ele é dado por

$$
\hat{T}_{\mu \nu}(x)=\operatorname{Tr} \int \mathrm{d}^{4} p\left[\hat{\Gamma}_{\mu \nu}(x, p)-\frac{1}{4} \eta_{\mu \nu} \hat{\Gamma}_{\lambda}^{\lambda}(x, p)\right]
$$

onde o traço se refere aos índices de cor.

Da mesma forma que no caso quântico anterior, as equações de transporte (4.36) para quarks e (4.45) para glúons devem ser resolvidas ordem a ordem no acoplamento $g$. Note que elas são explicitamente de ordem $g$ do lado direito, enquanto que o lado esquerdo contém um termo independente do acoplamento, o que permite um tratamento recursivo. O procedimento ficará mais claro no próximo capítulo, onde vamos considerar o caso da NCQED.

Mais recentemente tem havido grande interesse no estudo de soluções das equações de transporte para quarks e glúos apresentadas aqui, com aplicações diversas. Citamos os trabalhos de Litim, Manuel e Mrowczynski [61, 62] com estudos de plasmas quentes fracamente acoplados da QCD e ação efetiva, supercondutividade em quarks não-massivos e sistemas densos de quarks. Prozorkevich, Smolyansky e 
Ilyin [63] estudaram a criação de um plasma de quarks-antiquarks pela ação de campos intensos de glúons quase-clássicos, usando o formalismo de Wigner. Elze [64] estudou o comportamento dinâmico de poeira relativística quântica resolvendo equações de transporte quânticas com condições iniciais arbitrárias. Muitos outros trabalhos podem ser encontrados atualmente aplicando as equações de transporte a sistemas antes descritos apenas pela teoria de campos tradicional. 


\section{Capítulo 5}

\section{Equações de Transporte na QED Não-Comutativa}

Agora já temos tudo que precisamos para começar a fazer cálculos da QED não-comutativa usando ferramentas da teoria de transporte.

Conforme discutido no capítulo anterior, as equações de transporte se mostraram muito úteis para descrever o comportamento de plasmas da QCD no regime de altas temperaturas, ou limite HTL como vamos chamar aqui várias vezes por simplicidade de notação, que é o limite definido pela relação $p<<k \sim T$, onde $p$ representa um momento externo característico, $k$ denota o momento interno ao loop do diagrama e $T$ representa a temperatura do plasma. Neste limite sabemos que as amplitudes, ou funções de $n$-pontos, em 1-loop apresentam um comportamento dominante proporcional a $T^{2}$ e são todas independentes de gauge. No entanto, para levar em conta corretamente todos os termos dominantes que dão contribuição do tipo $T^{2}$ e que sejam invariantes de gauge, é necessário realizar um processo de "ressomação" das contribuições a altas temperaturas. Na QCD convencional este procedimento é tecnicamente complicado, e um método alternativo mais simples significaria um avanço considerável. Este método existe, é o método das equações de transportes clássicas, que fornecem resultados mais transparentes e mais diretos, pelo menos no limite HTL. Isto nos leva a perguntar se os mesmos métodos podem ser aplicados à QED não-comutativa, já que ela tem a mesma estrutura da QCD. A resposta não é óbvia, pois embora os resultados para os termos dominantes sejam corretos quando aplicados à QCD, sabemos que para os termos subdominantes isto não acontece. Talvez isto seja reflexo do fato de os termos subdominantes serem dependentes de gauge, e as equações de transportes serem manifestamente covariantes por transformações de gauge.

O comportamento de plasmas quentes em uma teoria não-comutativa tem suas peculiaridades, conforme descrito no capítulo 3. Vimos que, devido à presença do parâmetro de não-comutatividade, a aproximação de altas temperaturas tem duas regiões distintas onde os cálculos podem ser feitos analiticamente, que são as regiões $\pi \theta p T>>1$ e $\pi \theta p T<<1$, conforme discutido no final da seção 3.2.1. Vimos lá que a auto-energia do fóton na região $\pi \theta p T>>1$ é simplesmente proporcional a $T^{2}$, não havendo nenhuma dependência com o parâmetro de não-comutatividade, ou seja, o plasma se comporta como sendo livre de auto-interações. Já no limite $\pi \theta p T<<1$ o comportamento dominante é proporcional a $\theta$, da 
forma $T^{2}(\pi \theta p T)^{2}$, ou seja, o fator $T^{2}$ é suprimido pelo fator entre parênteses. Mesmo para uma teoria não-abeliana, como a teoria de Yang-Mills, definida no espaço não-comutativo, este comportamento se repete, e isso foi extensivamente estudado por Frenkel e colaboradores [46] no formalismo tradicional de diagramas de Feynman. O que vamos mostrar aqui é que estes resultados podem ser obtidos pelo método das equações de transporte, e mostrar que há concordância com os resultados obtidos no capítulo 3. Na primeira seção vamos apresentar a formulação clássica do problema, e discutir porque um tratamento quântico é necessário na discussão de campos de gauge (ou fótons). Este tratamento quântico é apresentado na segunda seção, onde é derivada uma equação de transporte apropriada para o caso dos fótons. Algumas dificuldades ainda persistem devido à não-comutatividade da teoria, e a solução é discutida na terceira seção, junto com uma equação de transporte simplificada que fornece corretamente os termos dominantes que estamos interessados.

\subsection{Tratamento clássico}

A idéia principal aqui é imaginar que as partículas do sistema são partículas clássicas que estão imersas em um banho térmico, formando um plasma quente cuja dinâmica é governada pelas equações de transporte clássicas. Isto vale também para as partículas movendo-se em um loop interno, que embora sejam partículas puramente quânticas, aqui elas serão tratadas como partículas clássicas cujas equações dinâmicas são aquelas de uma partícula na presença de um campo eletromagnético de fundo.

Sejam as equações de movimento clássicas de uma partícula sujeita a um campo eletromagnético dadas por

$$
\begin{aligned}
& m \frac{\mathrm{d} x^{\mu}}{\mathrm{d} \tau}=k^{\mu} \\
& m \frac{\mathrm{d} k^{\mu}}{\mathrm{d} \tau}=e F^{\mu}
\end{aligned}
$$

onde $\tau$ se refere ao tempo próprio da partícula e $F^{\mu}$ representa a força que ela sente na presença de um campo eletromagnético. A forma explícita de $F^{\mu}$ irá depender de ela ser carregada ou neutra, e os dois casos serão analisados separadamente. Em geral, a forma de $F^{\mu}$ deve ser tal que

$$
\begin{array}{r}
k^{2}=k^{\mu} k_{\mu}=m^{2} \\
F^{\mu} k_{\mu}=0
\end{array}
$$

a primeira condição indicando que a partícula deve estar na camada de massa e a segunda que a força é perpendicular ao seu movimento. Além disso a evolução temporal de $k^{\mu}$ deve se transformar covariantemente por uma transformação de gauge. Para um elétron por exemplo na presença de um campo eletromagnético usual, a forma explícita para a força é

$$
F^{\mu}=F^{\mu \nu} k_{\nu}
$$


Portanto, dadas as equações de movimento (5.1), podemos derivar a equação de transporte para a função distribuição $f(x, k)$ em analogia ao que foi feito no caso clássico da seção 4.1.

Assumindo por enquanto a natureza covariante da função distribuição, o análogo da equação de transporte (4.18) será da seguinte forma:

$$
D_{\tau} f(x, k)=\mathcal{C}
$$

onde $D_{\tau}$ é a derivada covariante ao longo da trajetória da partícula e $\mathcal{C}$, em geral, é um termo de colisão, em total analogia com (4.18). A forma explícita de $D_{\tau}$ é, no caso não-comutativo,

$$
D_{\tau} \equiv \frac{\mathrm{d}}{\mathrm{d} \tau}-i e \frac{\mathrm{d} x^{\mu}}{\mathrm{d} \tau}\left[A_{\mu},\right]_{\mathrm{MB}}
$$

e usando as equações de movimento (5.1) e a relação

$$
\frac{\mathrm{d} f}{\mathrm{~d} \tau}=\frac{\partial f}{\partial x^{\mu}} \frac{\mathrm{d} x^{\mu}}{\mathrm{d} \tau}+\frac{\partial f}{\partial k^{\mu}} \frac{\mathrm{d} k^{\mu}}{\mathrm{d} \tau},
$$

podemos escrever a equação de transporte (5.4) para $f(x, k)$ como

$$
k^{\mu} D_{\mu} f(x, k)+e F^{\mu} \star \frac{\partial f}{\partial k^{\mu}}=m \mathcal{C} .
$$

Esta é uma generalização natural para uma teoria não-comutativa da equação de transporte para a função distribuição $f$. Note que no limite comutativo usual a eletrodinâmica de Maxwell é recuperada e a equação de transporte para o caso de um plasma na ausência de termos de colisão se reduz, como esperado, à equação de Vlasov (4.20).

Em analogia com (4.7), vamos definir a corrente associada à partícula por

$$
j_{\mu}(x)=e \sum \int \mathrm{d} K k_{\mu} f(x, k)
$$

onde o sinal de somatório se refere a soma sobre as helicidades no caso de férmions e também sobre diferentes espécies de partículas se for o caso. A função distribuição é representada por $f(x, k)$ e a medida de integração é definida como

$$
\mathrm{d} K=\frac{\mathrm{d}^{4} k}{(2 \pi)^{3}} 2 \theta\left(k_{0}\right) \delta\left(k^{2}-m^{2}\right),
$$

onde a função $\theta\left(k_{0}\right)$ garante que a partícula tem energia positiva e a função $\delta$ garante que ela está na camada de massa. Note que em relação à (4.7) estamos adotando $c=1$ e o fator $1 /(2 \pi)^{3}$ foi introduzido apenas por simplicidade nos cálculos posteriores. Como veremos adiante esta corrente se conserva covariantemente como conseqüência do fato de que ela pertence à representação adjunta do grupo de gauge $U(1)$,

$$
D_{\mu} j^{\mu}=\partial_{\mu} j^{\mu}-i e\left[A_{\mu}, j^{\mu}\right]_{\mathrm{MB}}=0
$$

de maneira que a função distribuição também deve se transformar covariantemente. Mas a forma (5.7) para a equação de transporte é válida apenas no limite $\pi \theta p T>>1$, onde a força que age no sistema é dada 
efetivamente por (5.3) e o termo de colisão $\mathcal{C}$ é nulo. É somente neste limite que a conservação da corrente (5.10) é identicamente satisfeita.

Estamos interessados aqui no caso em que o parâmetro $\theta$ possa assumir qualquer valor, de maneira que um termo de colisão deve estar presente, mesmo na ordem mais baixa dos cálculos. Isto se deve ao fato de a teoria não-comutativa ser auto-interagente, então devemos ter uma forma mais complicada para a força na equação de movimento (5.1), de maneira a levar em conta a não-comutatividade. Isso deve dar origem inevitavelmente a um termo de colisão. Baseado nisso, é fácil verificar que um termo de colisão do tipo

$$
\mathcal{C}=-\frac{e}{m} \frac{\partial F^{\mu}}{\partial k^{\mu}} \star f(x, k)
$$

leva corretamente à conservação da corrente (5.10). Antes de demonstrarmos isto, note que a equação de transporte (5.7) com este termo de colisão pode finalmente ser escrita como

$$
k^{\mu} D_{\mu} f(x, k)+\frac{\partial\left(e F^{\mu} \star f(x, k)\right)}{\partial k^{\mu}}=0 .
$$

A conservação da corrente pode ser demonstrada como segue. Aplicando a derivada covariante à corrente e usando a equação de transporte (5.12) obtemos

$$
\begin{aligned}
D^{\mu} j_{\mu} & =e \sum \int \mathrm{d} K k_{\mu} D^{\mu} f(x, k) \\
& =-e^{2} \sum \int \frac{\mathrm{d}^{4} k}{(2 \pi)^{3}} 2 \theta\left(k_{0}\right) \delta\left(k^{2}-m^{2}\right) \frac{\partial\left(F^{\mu} \star f(x, k)\right)}{\partial k^{\mu}} \\
& =e^{2} \sum \int \frac{\mathrm{d}^{4} k}{(2 \pi)^{3}} 2 \theta\left(k_{0}\right) 2 k_{\mu} F^{\mu} \star f(x, k) \delta^{\prime}\left(k^{2}-m^{2}\right) \\
& =0
\end{aligned}
$$

onde na penúltima igualdade fizemos uma integração por partes e $\delta^{\prime}$ significa a derivada da função $\delta$ com relação ao seu argumento. Na última linha usamos a condição (5.2) que a força deve satisfazer.

Para continuarmos os cálculos devemos agora supor uma forma explícita para a força agindo na partícula. Isto vai depender dela ser carregada (elétrons) ou neutra (fótons).

\subsubsection{Partículas carregadas - elétrons}

Para partículas carregadas, como o elétron por exemplo, a forma usual de se escrever a equação de força em termos do tensor eletromagnético $F^{\mu \nu}$ é

$$
F^{\mu}=F^{\mu \nu} k_{\nu}
$$

de maneira que obtemos $\mathcal{C}=0$ da equação (5.11), pois $F_{\mu}^{\mu}=0$, o que significa que estamos falando de um plasma livre de colisões. No entanto, se a força tiver uma dependência um pouco mais complicada em $k^{\mu}$, como parece ser o caso quando tratamos da QED não-comutativa, haverá inevitavelmente uma contribuição devida ao termo de colisão, e esta contribuição deve estar presente se quisermos tratar de maneira correta 
um plasma não-comutativo a altas temperaturas. De fato, como sabemos, a teoria não-comutativa tem uma estrutura característica de dipolos, de maneira que é natural generalizar a expressão da força para incluir além do termo (5.14), um termo de dipolo. A proposta dos autores ${ }^{1}$ foi então uma força da forma

$$
F^{\mu}=(1+\sin k \times i D) F^{\mu \nu} k_{\nu},
$$

onde $k \times i D=i k_{j} \theta^{j l} D_{l}$. O primeiro termo é a força de Lorentz usual para uma partícula pontual carregada. O segundo termo pode ser pensado como uma interação de dipolo, sendo uma generalização natural, considerando-se que partículas não-comutativas apresentam uma característica de dipolos. Além do mais, este termo se anula quando $\theta^{\mu \nu} \rightarrow 0$, reduzindo naturalmente à teoria convencional para uma partícula carregada. A presença deste termo de dipolo dará uma contribuição não-trivial vinda de $\mathcal{C}$, mas já sabemos dos resultados do capítulo 3 que uma contribuição deste tipo não está presente nas amplitudes em ordem dominante a altas temperaturas. Portanto, no limite que estamos interessados, é suficiente tomar apenas o primeiro termo da força, de maneira que a equação de transporte que temos de resolver é da forma

$$
k^{\mu} D_{\mu} f(x, k)+\frac{\partial\left(e k_{\nu} F^{\mu \nu} \star f(x, k)\right)}{\partial k^{\mu}}=0
$$

onde, no limite HTL, temos efetivamente,

$$
F^{\mu}=F^{\mu \nu} k_{\nu}
$$

e vemos que a equação (5.16) tem a forma parecida com a da equação de transporte para a QED usual, sendo a única diferença o produto Moyal que leva em conta a não-comutatividade espacial.

Vamos então demonstrar brevemente que a equação de transporte acima é a correta para obtermos as amplitudes de $n$-pontos em 1-loop no limite de altas temperaturas. Em primeiro lugar escrevemos a função distribuição expandida em potências do acoplamento $e$,

$$
f(x, k)=f^{(0)}(x, k)+e f^{(1)}(x, k)+e^{2} f^{(2)}(x, k)+\cdots
$$

onde só conhecemos $f^{(0)}$, dado pela função distribuição de Fermi-Dirac,

$$
f^{(0)}(x, k) \sim n_{F}\left(k_{0}\right)=\frac{1}{\mathrm{e}^{k_{0} / T}+1} .
$$

Então substituímos a função distribuição expandida na equação de transporte (5.16), e obtemos para $f^{(1)}(x, k)$

$$
k \cdot \partial f^{(1)}(x, k)=\frac{\partial}{\partial k_{\nu}}\left(\left(k \cdot \partial A_{\nu}-\partial_{\nu} k \cdot A\right) \star f^{(0)}(x, k)\right) .
$$

Escrevemos $f^{(1)}(x, k)$ e $A^{\mu}(x)$ como transformadas de Fourier,

$$
f^{(1)}(x, k)=\int \mathrm{d}^{4} p f^{(1)}(p, k) \mathrm{e}^{i p \cdot x}
$$

${ }^{1}$ Frenkel, Das e Brandt, conforme eq. (29) da ref. [68]. 


$$
A^{\mu}(x)=\int \mathrm{d}^{4} p A^{\mu}(p) \mathrm{e}^{i p \cdot x}
$$

substituímos em (5.20) e obtemos $f^{(1)}(p, k)$,

$$
f^{(1)}(p, k)=\frac{1}{k \cdot p} \frac{\partial}{\partial k_{\nu}}\left(\left(k \cdot p A_{\nu}(p)-p_{\nu} k \cdot A(p)\right) f^{(0)}\right)
$$

Note que $f^{(0)}$ não depende de $x$, por isso não é mais necessário usar o produto Moyal. Usando a expansão (5.18) para $f$, a corrente fica

$$
j_{\mu}(x)=e \sum \int \frac{\mathrm{d}^{4} k}{(2 \pi)^{3}} 2 \theta\left(k_{0}\right) \delta\left(k^{2}-m^{2}\right) k_{\mu}\left[f^{(0)}(x, k)+e f^{(1)}(x, k)+e^{2} f^{(2)}(x, k)+\cdots\right]
$$

de maneira que, calculando cada termo de $f$ por meio da equação de transporte, a corrente fica completamente determinada também ordem a ordem no parâmetro $e$. Por exemplo, a corrente em ordem $e^{2}$, que dará contribuição para a amplitude de 2-pontos, é dada em termos de $f^{(1)}$ por

$$
j_{\mu}^{(2)}(x)=e^{2} \sum \int \frac{\mathrm{d}^{4} k}{(2 \pi)^{3}} 2 \theta\left(k_{0}\right) \delta\left(k^{2}-m^{2}\right) k_{\mu} f^{(1)}(x, k) .
$$

Escrevemos então a corrente como transformada de Fourier

$$
j_{\mu}^{(2)}(x)=\int \mathrm{d}^{4} p j_{\mu}^{(2)}(p) \mathrm{e}^{i p \cdot x}
$$

de maneira que, usando (5.21) e (5.23) obtemos

$$
j_{\mu}^{(2)}(p)=e^{2} \sum \int \frac{\mathrm{d}^{4} k}{(2 \pi)^{3}} 2 \theta\left(k_{0}\right) \delta\left(k^{2}-m^{2}\right) \frac{k_{\mu}}{k \cdot p} \frac{\partial}{\partial k_{\nu}}\left(\left(k \cdot p A_{\nu}(p)-p_{\nu} k \cdot A(p)\right) f^{(0)}\right) .
$$

Integrando por partes obtemos

$$
\begin{gathered}
j_{\mu}^{(2)}(p)=-e^{2} \sum \int \frac{\mathrm{d}^{4} k}{(2 \pi)^{3}} 2 \theta\left(k_{0}\right) \delta\left(k^{2}-m^{2}\right) \frac{\partial}{\partial k_{\nu}}\left[\frac{k_{\mu}}{k \cdot p}\right]\left(k \cdot p A_{\nu}(p)-p_{\nu} k \cdot A(p)\right) f^{(0)} \\
=-e^{2} \sum \int \frac{\mathrm{d}^{4} k}{(2 \pi)^{3}} 2 \theta\left(k_{0}\right) \delta\left(k^{2}-m^{2}\right)\left[\eta_{\mu}^{\nu} A_{\nu}(p)-\frac{p_{\mu}}{k \cdot p} k \cdot A(p)-\frac{k_{\mu}}{k \cdot p} p \cdot A(p)\right. \\
\left.+\frac{k_{\mu}}{(k \cdot p)^{2}} p^{2} k \cdot A(p)\right] f^{(0)}
\end{gathered}
$$

onde $f^{(0)}$ é dado por (5.19). No limite de altas temperaturas podemos desprezar a massa da partícula, e a 
medida de integração fica

$$
\frac{\mathrm{d}^{4} k}{(2 \pi)^{3}} 2 \theta\left(k_{0}\right) \delta\left(k^{2}\right)=\frac{\mathrm{d}^{3} k}{(2 \pi)^{3}} \frac{1}{|\vec{k}|}
$$

A somatória é sobre os spins, $(2 s+1)$, portanto fornece um fator 2. Desta forma a função de 2-pontos para o loop fermiônico pode ser determinada por meio de

$$
\begin{aligned}
\Pi_{\mu \nu}^{(\text {fermion })}(p) & =\left.\frac{\delta j_{\mu}(p)}{\delta A^{\nu}(-p)}\right|_{A_{\mu}=0}=\frac{\delta j_{\mu}^{(2)}(p)}{\delta A^{\nu}(-p)} \\
& =-\left.\frac{8 e^{2}}{(2 \pi)^{3}} \int \frac{\mathrm{d}^{3} k}{|\vec{k}|} n_{F}(|\vec{k}|)\left[\eta_{\mu \nu}-\frac{p_{\mu} k_{\nu}+k_{\mu} p_{\nu}}{k \cdot p}+\frac{k_{\mu} k_{\nu} p^{2}}{(k \cdot p)^{2}}\right]\right|_{k^{0}=|\vec{k}|},
\end{aligned}
$$

que é exatamente o mesmo resultado obtido em (3.68) no capítulo 3, pelo método da amplitude de espalhamento frontal.

Como demonstramos acima, a equação de transporte (5.16) fornece corretamente a primeira correção à função distribuição $f^{(0)}$ no equilíbrio. Com ela a corrente até ordem $e^{2}$ pode ser calculada e a função de 2-pontos fica completamente determinada para um loop fermiônico no limite de altas temperaturas. Da mesma forma, utilizando a equação de transporte (5.16), podemos determinar a correção $f^{(2)}$ à função distribuição e por meio dela determinar a corrente até ordem $e^{3}$ e assim obter a função de 3-pontos, conforme demonstrado em [68].

\subsubsection{Partículas neutras - fótons}

Vamos agora tratar do caso de partículas neutras, como o fóton por exemplo, que como vimos no capítulo 3 tem sua auto-energia modificada devido exclusivamente à não-comutatividade das coordenadas. $\mathrm{O}$ caso das equações de transporte para partículas neutras é muito mais interessante, pois como sabemos, uma partícula neutra usual não sofre nenhum tipo de força eletromagnética. Mas no caso não-comutativo não é bem assim por causa da estrutura "estendida" das partículas devido à não-comutatividade. Desta forma, mesmo uma partícula neutra pode ter uma natureza do tipo dipolo, e assim sentir alguma força devido a algum campo eletromagnético externo. Mas também sabemos que, como visto na seção anterior, a natureza dipolar devido ao parâmetro de não-comutatividade $\theta$ não está presente no cálculo de termos dominantes. Então, já que a estrutura de dipolo não dá qualquer contribuição na ordem dominante, podemos pensar que uma estrutura do tipo quadrupolo possa dar alguma contribuição. De fato é isto que acontece. A forma da força para o caso de partículas neutras proposta pelos mesmos autores da discussão anterior se reduz à uma interação do tipo quadrupolo no limite de baixos momentos. Ela é dada por ${ }^{2}$

$$
X_{\text {(neutra) }}^{\mu}=2\left\{1-\cos k \times\left(i D+e\left[\frac{1}{k \cdot D} F,\right]_{M B}\right)\right\} F^{\mu \nu} k_{\nu}
$$

\footnotetext{
${ }^{2}$ Ver eq. (38) da ref. [68].
} 
Vemos que no limite $\theta \rightarrow 0$ a força se anula, como deve ser para uma partícula neutra. Substituindo esta força na equação de transporte (5.12) e usando os mesmos passos da seção anterior, obtemos a correção $f^{(1)}$ à função distribuição, dada por

$$
f^{(1)}=\frac{2}{k \cdot \partial} \frac{\partial}{\partial k_{\mu}}\left((1-\cos k \times i \partial)\left(k \cdot \partial A_{\mu}-\partial_{\mu} k \cdot A\right) f^{(0)}(x, k)\right)
$$

onde agora

$$
f^{(0)}(x, k) \sim n_{B}\left(k_{0}\right)=\frac{1}{\mathrm{e}^{k_{0} / T}-1}
$$

é a função distribuição de Bose-Einstein. Com isto calculamos a corrente em ordem $e^{2}$ e finalmente a função de 2-pontos, ou auto-energia do fóton, dada por

$$
\begin{aligned}
\Pi_{\mu \nu}^{\text {(gauge) }}(p) & =\left.\frac{\delta j_{\mu}(p)}{\delta A^{\nu}(-p)}\right|_{A_{\mu}=0} \\
& =-\frac{4 e^{2}}{(2 \pi)^{3}} \int \frac{\mathrm{d}^{3} k}{|\vec{k}|} n_{B}(|\vec{k}|)(1-\cos p \times k)\left[\eta_{\mu \nu}-\frac{p_{\mu} k_{\nu}+k_{\mu} p_{\nu}}{k \cdot p}+\frac{k_{\mu} k_{\nu} p^{2}}{(k \cdot p)^{2}}\right] .
\end{aligned}
$$

Os cálculos são exatamente semelhantes ao do caso anterior, a única diferença sendo a presença do fator trigonométrico que caracteriza a não-comutatividade. Este resultado é o mesmo obtido em (3.65) no capítulo 3. Da mesma forma, pode-se obter a correção $f^{(2)}$ à função distribuição, e com ela a função de 3-pontos no limite de altas temperaturas. Isto está feito em [68], demonstrando que a equação de transporte para uma partícula neutra está de fato correta, assim como a forma da força (5.31) para uma partícula neutra na NCQED.

Olhando para a equação de transporte (5.12), vemos que, para o caso da força (5.31), ela pode ser escrita como

$$
k^{\mu} D_{\mu} f(x, k)=-e \frac{\partial\left(X^{\mu} \star f(x, k)\right)}{\partial k^{\mu}},
$$

e a força é, em primeira aproximação, dada por

$$
X_{(\text {neutra })}^{\mu} \simeq 2[1-\cos k \times i \partial] F^{\mu \nu} k_{\nu}
$$

\subsection{Tratamento quântico - O operador de Wigner para os fótons}

$\mathrm{Na}$ dificuldade em se justificar a forma explícita para a força que age em uma partícula neutra com o tratamento clássico da seção anterior, os autores procuraram por uma nova formulação para o problema, seguindo o método quântico apresentado no capítulo anterior, que diz respeito ao método de Wigner de se formular as equações de transporte. O objetivo é formular a função distribuição quântica do problema, que é a função de Wigner, cuja equação de evolução segue diretamente das equações satisfeitas pelos campos correspondentes. Portanto não é necessário conhecer a forma da força que age na partícula, como acontece no tratamento clássico. No entanto, no caso de campos auto-interagentes, como é o caso da QED 
não-comutativa, devemos tomar cuidado com duas coisas. A primeira é que a função de Wigner deve ser formulada de uma maneira covariante de gauge, e a segunda é que devemos usar aproximações de campo médio apropriadas para extrair o conteúdo físico do sistema.

Apresentaremos aqui brevemente as principais idéias para se chegar a uma equação de transporte conveniente para a NCQED. Estes resultados foram apresentados por Frenkel, Brandt e Das em [69], em estreita analogia com a formulação apresentada na seção 4.2.4 para campos de gauge não-abelianos. Os cálculos explícitos das amplitudes de 2 e 3-pontos serão feitos na seção seguinte, utilizando-se uma forma mais simples para a equação de transporte que a apresentada aqui, derivada posteriormente.

Já que o campo para o fóton não-comutativo apresenta a mesma estrutura auto-interagente dos glúons da QCD usual, define-se, para os fótons não-comutativos, um operador de Wigner ${ }^{3}$ covariante de gauge análogo a (4.42),

$$
W_{\mu \nu}(x, k)=\int \frac{\mathrm{d}^{4} y}{(2 \pi \hbar)^{4}} e^{-\frac{i}{\hbar} y \cdot k} G_{\mu \lambda}^{(+)}(x) \star G_{\nu}^{\lambda(-)}(x)
$$

onde definimos também

$$
G_{\mu \nu}^{( \pm)}(x)=U\left(x, x_{ \pm}\right) \star F_{\mu \nu}\left(x_{ \pm}\right) \star U\left(x_{ \pm}, x\right)
$$

e $U$ representa o operador de link para a teoria não-comutativa, definido ao longo de uma trajetória retilínea,

$$
U\left(x, x_{ \pm}\right)=P\left(\mathrm{e}_{\star}^{\mp \frac{i e}{\hbar c} \int_{0}^{1} d t \frac{y}{2} \cdot A\left(x \pm(1-t) \frac{y}{2}\right)}\right)
$$

e $P$ indica ordenamento da esquerda para a direita, e pode-se checar que por uma transformação de gauge do tipo (2.55) o operador de link (5.39) se transforma covariantemente,

$$
U\left(x, x_{ \pm}\right) \rightarrow \Omega^{-1}(x) \star U\left(x, x_{ \pm}\right) \star \Omega\left(x_{ \pm}\right) .
$$

Note que, com o operador de link definido desta maneira, podemos também escrever

$$
G_{\mu \nu}^{( \pm)}(x)=\left(\mathrm{e}_{\star}^{ \pm \frac{y}{2} \cdot D} F_{\mu \nu}(x)\right)
$$

onde a derivada covariante deve ser definida na representação adjunta. Também é fácil checar que

$$
W_{\mu \nu}^{\dagger}(x, k)=W_{\nu \mu}(x, k)
$$

A derivação da equação de transporte para a função de Wigner (5.37) se faz da seguinte maneira. Precisamos calcular a quantidade $k \cdot D W_{\mu \nu}(x, k)$, e para isto precisaremos das relações abaixo:

$$
\begin{aligned}
D_{\mu}^{(x)} U\left(x, x_{ \pm}\right) & =\partial_{\mu}^{(x)} U\left(x, x_{ \pm}\right)-\frac{i e}{\hbar c}\left(A_{\mu}(x) \star U\left(x, x_{ \pm}\right)-U\left(x, x_{ \pm}\right) \star A_{\mu}\left(x_{ \pm}\right)\right) \\
& =\mp \frac{i e}{2 \hbar c} y^{\nu}\left(\int_{0}^{1} \mathrm{~d} t\left(e_{\star}^{ \pm \frac{t y}{2} \cdot D} F_{\mu \nu}(x)\right)\right) \star U\left(x, x_{ \pm}\right)
\end{aligned}
$$

\footnotetext{
${ }^{3}$ Por simplicidade de notação, vamos omitir o símbolo operatorial daqui para frente, de forma que $\hat{W} \rightarrow W$, etc.
} 


$$
\begin{aligned}
& D_{\mu}^{(x)} U\left(x_{ \pm}, x\right)=\partial_{\mu}^{(x)} U\left(x_{ \pm}, x\right)-\frac{i e}{\hbar c}\left(A_{\mu}\left(x_{ \pm}\right) \star U\left(x_{ \pm}, x\right)-U\left(x_{ \pm}, x\right) \star A_{\mu}(x)\right) \\
&= \pm \frac{i e}{2 \hbar c} y^{\nu} U\left(x_{ \pm}, x\right) \star\left(\int_{0}^{1} \mathrm{~d} t\left(e_{\star}^{ \pm \frac{t y}{2} \cdot D} F_{\mu \nu}(x)\right)\right) \\
& D_{\rho}^{(x)} G_{\mu \nu}^{( \pm)}(x)=\left(D_{\rho}^{(x)} U\left(x, x_{ \pm}\right)\right) \star F_{\mu \nu}\left(x_{ \pm}\right) \star U\left(x_{ \pm}, x\right) \\
&+ U\left(x, x_{ \pm}\right) \star\left(D_{\rho}^{(x)} F_{\mu \nu}\left(x_{ \pm}\right) \star U\left(x_{ \pm}, x\right)\right. \\
&+ U\left(x, x_{ \pm}\right) \star F_{\mu \nu}\left(x_{ \pm}\right) \star\left(D_{\rho}^{(x)} U\left(x_{ \pm}, x\right)\right)
\end{aligned}
$$

Com isto pode-se mostrar que a função de Wigner na QED não-comutativa deve satisfazer a seguinte equação de transporte:

$$
\begin{aligned}
& k \cdot D W_{\mu \nu}(x, k)= \\
& =\frac{e}{2 c} \frac{\partial}{\partial k_{\sigma}} k^{\rho}\left[\left(\int_{0}^{1} \mathrm{~d} t\left(\mathrm{e}_{\star}^{\frac{i \hbar t}{2} \partial_{k} \cdot D} F_{\rho \sigma}(x)\right)\right) \star W_{\mu \nu}+W_{\mu \nu} \star\left(\int_{0}^{1} \mathrm{~d} t\left(\mathrm{e}_{\star}^{-\frac{i \hbar t}{2} \partial_{k} \cdot D} F_{\rho \sigma}(x)\right)\right)\right. \\
& \left.-\int \frac{\mathrm{d}^{4} y}{(2 \pi \hbar)^{4}} \mathrm{e}^{-\frac{i}{\hbar} y \cdot k} G_{\mu \lambda}^{(+)}(x) \star\left(\int_{0}^{1} d t\left[\left(\mathrm{e}_{\star}^{\frac{t y}{2} \cdot D} F_{\rho \sigma}(x)\right)+\left(\mathrm{e}_{\star}^{-\frac{t y}{2} \cdot D} F_{\rho \sigma}(x)\right)\right]\right) \star G_{\nu}^{\lambda(-)}(x)\right] \\
& +k^{\rho} \int \frac{\mathrm{d}^{4} y}{(2 \pi \hbar)^{4}} e^{-\frac{i}{\hbar} y \cdot k}\left[U\left(x, x_{+}\right) \star\left(D_{\rho}^{\left(x_{+}\right)} F_{\mu \lambda}\right)\left(x_{+}\right) \star U\left(x_{+}, x\right) \star G_{\nu}^{\lambda(-)}(x)\right. \\
& \left.+G_{\mu \lambda}^{(+)}(x) \star U\left(x, x_{-}\right) \star\left(D_{\rho}^{\left(x_{-}\right)} F_{\nu}^{\lambda}\right)\left(x_{-}\right) \star U\left(x_{-}, x\right)\right]
\end{aligned}
$$

onde $\partial_{k}$ representa a derivada com relação a $k$. Esta equação representa a equação de transporte completa para a função de Wigner dos fótons da QED não-comutativa e sua solução geral é muito difícil de ser obtida. No entanto, como estamos interessados apenas no limite de altas temperaturas, ou limite HTL, pode-se mostrar que neste limite a equação de transporte assume a forma mais simples,

$$
\begin{aligned}
k \cdot D W_{\mu \nu}(x, k)=\frac{e}{2 c} & \frac{\partial}{\partial k_{\sigma}} k^{\rho}\left[F_{\rho \sigma}(x) \star W_{\mu \nu}(x, k)+W_{\mu \nu}(x, k) \star F_{\rho \sigma}(x)\right. \\
& \left.-2 \int \frac{\mathrm{d}^{4} y}{(2 \pi \hbar)^{4}} e^{-\frac{i}{\hbar} y \cdot k} G_{\mu \lambda}^{(+)}(x) \star F_{\rho \sigma}(x) \star G_{\nu}^{\lambda(-)}(x)\right]
\end{aligned}
$$

conforme discutido em [69].

Sendo a QED não-comutativa uma teoria auto-interagente devemos, da mesma forma que na QCD, separar os campos em uma parte quântica $a_{\mu}$, e outra parte em campo de fundo $\bar{A}_{\mu}$, fazendo uma decomposição da forma

$$
A_{\mu}(x)=\bar{A}_{\mu}(x)+a_{\mu}(x)
$$

onde

$$
a^{\mu}(x)=\sum_{s} \int \frac{\mathrm{d}^{3} k}{(2 \pi)^{3} 2 k^{0}} \epsilon^{\mu}\left(a \mathrm{e}^{-i k \cdot x}+a^{\dagger} \mathrm{e}^{i k \cdot x}\right),
$$

é a solução de ondas planas para o campo de gauge quântico. Assumimos que na aproximação de campo 
médio valem as relações

$$
\left\langle A_{\mu}(x)\right\rangle=\bar{A}_{\mu}(x), \quad\left\langle a_{\mu}(x)\right\rangle=0
$$

de maneira que por uma transformação de gauge os campos se transformam como

$$
\begin{gathered}
\bar{A}_{\mu}(x) \rightarrow \Omega^{-1}(x) \star \bar{A}_{\mu}(x) \star \Omega(x)+\frac{i \hbar c}{e} \Omega^{-1}(x) \star \partial_{\mu} \Omega(x), \\
a_{\mu}(x) \rightarrow \Omega^{-1}(x) \star a_{\mu}(x) \star \Omega(x) .
\end{gathered}
$$

Além disto, nesta decomposição temos

$$
F_{\mu \nu}(x)=\bar{F}_{\mu \nu}(x)+\bar{D}_{\mu} a_{\nu}(x)-\bar{D}_{\nu} a_{\mu}(x)-\frac{i e}{\hbar c}\left[a_{\mu}(x), a_{\nu}(x)\right]_{\mathrm{MB}}
$$

onde

$$
\begin{gathered}
\bar{D}_{\mu} a_{\nu}=\partial_{\mu} a_{\nu}-\frac{i e}{\hbar c}\left[\bar{A}_{\mu}, a_{\nu}\right]_{\mathrm{MB}} \\
\bar{F}_{\mu \nu}=\partial_{\mu} \bar{A}_{\nu}-\partial_{\nu} \bar{A}_{\mu}-\frac{i e}{\hbar c}\left[\bar{A}_{\mu}, \bar{A}_{\nu}\right]_{\mathrm{MB}}
\end{gathered}
$$

Definindo então

$$
\mathcal{G}_{\mu \nu}(x, k)=\left\langle W_{\mu \nu}(x, k)\right\rangle-\bar{W}_{\mu \nu}(x, k)
$$

onde $\bar{W}_{\mu \nu}(x, k)$ representa a função de Wigner associada com o campo de fundo $\bar{A}^{\mu}$, podemos escrever a equação de transporte para $\mathcal{G}_{\mu \nu}(x, k)$ na aproximação de altas temperaturas como

$$
\begin{aligned}
& k \cdot \bar{D} \mathcal{G}_{\mu \nu}(x, k)=\frac{e}{2 c} \frac{\partial}{\partial k_{\sigma}} k^{\rho}\left\{\bar{F}_{\rho \sigma}(x) \star \mathcal{G}_{\mu \nu}(x, k)+\mathcal{G}_{\mu \nu}(x, k) \star \bar{F}_{\rho \sigma}(x)\right. \\
& \left.-2 \int \frac{\mathrm{d}^{4} y}{(2 \pi \hbar)^{4}} e^{-\frac{i}{\hbar} y \cdot k}\left[\left\langle G_{\mu \lambda}^{(+)}(x) \star \bar{F}_{\rho \sigma}(x) \star G_{\nu}^{\lambda(-)}(x)\right\rangle-\left(\bar{G}_{\mu \lambda}^{(+)}(x) \star \bar{F}_{\rho \sigma}(x) \star \bar{G}_{\nu}^{\lambda(-)}(x)\right)\right]\right\} .
\end{aligned}
$$

Se definirmos então a quantidade

$$
\mathcal{F}(x, k)=\frac{1}{k^{2}} \eta^{\mu \nu} \mathcal{G}_{\mu \nu}(x, k),
$$

ela deve satisfazer a seguinte equação:

$$
\begin{aligned}
& k \cdot \bar{D} \mathcal{F}_{\mu \nu}(x, k)=\frac{e}{2 c} \frac{\partial}{\partial k_{\sigma}} k^{\rho}\left\{\bar{F}_{\rho \sigma}(x) \star \mathcal{F}_{\mu \nu}(x, k)+\mathcal{F}_{\mu \nu}(x, k) \star \bar{F}_{\rho \sigma}(x)\right. \\
& \left.-2 \int \frac{\mathrm{d}^{4} y}{(2 \pi \hbar)^{4}} e^{-\frac{i}{\hbar} y \cdot k} \frac{1}{k^{2}}\left[\left\langle G_{\mu \lambda}^{(+)}(x) \star \bar{F}_{\rho \sigma}(x) \star G_{\nu}^{\lambda(-)}(x)\right\rangle-\left(\bar{G}_{\mu \lambda}^{(+)}(x) \star \bar{F}_{\rho \sigma}(x) \star \bar{G}_{\nu}^{\lambda(-)}(x)\right)\right]\right\},
\end{aligned}
$$

que é manifestamente covariante por transformações de gauge e pode ser resolvida ordem a ordem no 
parâmetro e para dar o valor médio da função de Wigner. Se definirmos a corrente como

$$
\begin{aligned}
J_{\mu}(x) & =\frac{e}{2} \int \mathrm{d}^{4} k \theta\left(k^{0}\right) \frac{k_{\mu}}{k^{2}} \eta^{\lambda \rho}\left(\mathcal{G}_{\lambda \rho}(x, k)-\mathcal{G}_{\rho \lambda}(x,-k)\right) \\
& =\frac{e}{2} \int \mathrm{d}^{4} k \theta\left(k^{0}\right) k_{\mu}(\mathcal{F}(x, k)-\mathcal{F}(x,-k))
\end{aligned}
$$

é fácil verificar que esta corrente se transforma corretamente por uma transformação de gauge e é covariantemente conservada

$$
\bar{D}_{\mu} J^{\mu}(x)=\partial_{\mu} J^{\mu}-\frac{i e}{\hbar c}\left[\bar{A}_{\mu}, J^{\mu}\right]_{M B}=0
$$

Da mesma forma que no tratamento da equação de Schrödinger da seção 4.2.1, podemos ver que o lado direito já é explicitamente de ordem $e$, enquanto que o lado esquerdo contém um termo independente, de forma que $\mathcal{F}$ pode ser obtida ordem a ordem no parâmetro $e$, e então a corrente (5.56) também pode ser obtida ordem a ordem, e as funções de $n$-pontos podem ser determinadas por meio de

$$
\Gamma^{\mu_{1} \mu_{2} \cdots \mu_{n}}\left(p_{1}, \cdots, p_{n}\right)=\left.\frac{\delta^{n-1} J^{\mu_{1}}\left(-p_{1}\right)}{\delta \bar{A}_{\mu_{2}}\left(p_{2}\right) \cdots \delta \bar{A}_{\mu_{n}}\left(p_{n}\right)}\right|_{\bar{A}=0} .
$$

A equação de transporte (5.55) é o principal resultado. Junto com a corrente (5.56) podemos obter todas as funções de $n$-pontos da mesma forma que no caso clássico anterior. Não faremos esta demonstração aqui porque queremos ainda ir um pouco além e apresentar uma forma mais simples para função de Wigner e para a equação de transporte, com a qual calcularemos as funções de $n$-pontos. Vamos terminar dizendo o que nos motivou a procurar uma forma mais simples para a função de Wigner. Em primeiro lugar note que a definição (5.54) é singular, pois a princípio $k^{2}=0$ deve estar na camada de massa. Este termo vai se cancelar apenas posteriormente, quando aparecer no numerador um termo proporcional a $k^{2}$, mas até então ela não faz muito sentido. Outra coisa que se percebeu depois é que para obter corretamente as contribuições para as amplitudes de 3 e 4-pontos era necessário incluir uma contribuição de ordem $e$ vinda da quantização correta do campo $a^{\mu}(x)$ dado em (5.47), pois para o caso não-comutativo aquela não é a expressão correta, como se percebeu depois. Isto foi chamado de covariantização em [69], pois ainda não estava claro que a solução viria desta quantização. Isto foi feito no trabalho posterior, o qual apresentamos na sessão seguinte.

\subsection{Forma simplificada da função de Wigner para fótons}

Nesta última parte vamos ver em detalhes como uma forma mais simples para a função de Wigner leva corretamente às amplitudes de 2 e 3-pontos em 1-loop da QED não-comutativa a altas temperaturas. Devido a algumas dificuldades já citadas com a formulação anterior, propomos a seguinte forma para a 
função de Wigner $w_{\mu \nu}(x, k)$, válida para a aproximação de altas temperaturas, ou aproximação HTL:

$$
w_{\mu \nu}(x, k)=\int \frac{\mathrm{d}^{4} y}{(2 \pi)^{4}} e^{-i y \cdot k} G_{\mu}^{(+)}(x) \star G_{\nu}^{(-)}(x),
$$

onde

$$
G_{\mu}^{( \pm)}(x)=U^{(\bar{A})}\left(x, x_{ \pm}\right) \star a_{\mu}\left(x_{ \pm}\right) \star U^{(\bar{A})}\left(x_{ \pm}, x\right) .
$$

com $x_{ \pm}=x \pm \frac{y}{2}$ e os operadores de link $U$ são dados por

$$
\begin{aligned}
& U^{(\bar{A})}\left(x, x_{ \pm}\right)=P \mathrm{e}^{\mp \frac{i e}{\hbar c} \int_{0}^{1} d s \frac{y}{2} \cdot \bar{A}\left(x \pm(1-s) \frac{y}{2}\right)}, \\
& U^{(\bar{A})}\left(x_{ \pm}, x\right)=P \mathrm{e}^{ \pm \frac{i e}{\hbar c} \int_{0}^{1} d s \frac{y}{2} \cdot \bar{A}\left(x \pm s \frac{y}{2}\right)} .
\end{aligned}
$$

A função distribuição é dada por

$$
\mathcal{F}(x, k)=\eta^{\mu \nu}\left\langle w_{\mu \nu}(x, k)\right\rangle,
$$

evitando assim o problema do fator $1 / k^{2}$ presente na (5.54). A equação de transporte a ser satisfeita por $\mathcal{F}$ é dada simplesmente por

$$
k \cdot \bar{D} \mathcal{F}(x, k)=\frac{e}{2} \frac{\partial}{\partial k_{\sigma}} k^{\rho}\left[\bar{F}_{\rho \sigma} \star \mathcal{F}+\mathcal{F} \star \bar{F}_{\rho \sigma}-2 \int \frac{\mathrm{d}^{4} y}{(2 \pi)^{4}} e^{-i y \cdot k}\left\langle G_{\mu}^{(+)} \star \bar{F}_{\rho \sigma} \star G^{\mu(-)}\right\rangle\right],
$$

com a derivada covariante $\bar{D}_{\mu}=\partial_{\mu}-i e[\bar{A},]_{M B}$. Resolvendo iterativamente esta equação para $\mathcal{F}$ expandido em potências da constante de acoplamento $e$,

$$
\mathcal{F}(x, k)=\mathcal{F}^{(0)}(x, k)+e \mathcal{F}^{(1)}(x, k)+e^{2} \mathcal{F}^{(2)}(x, k)+\cdots
$$

vamos obter uma corrente

$$
J_{\mu}(x)=-e \int \mathrm{d}^{4} k k_{\mu}[\mathcal{F}(x, k)-\mathcal{F}(x,-k)]
$$

para todas as ordens em $e$, com a qual calculamos as funções de $n$-pontos

$$
\Gamma^{\mu_{1} \mu_{2} \cdots \mu_{n}}\left(p_{1}, \cdots, p_{n}\right)=\left.\frac{\delta^{n-1} J^{\mu_{1}}\left(-p_{1}\right)}{\delta \bar{A}_{\mu_{2}}\left(p_{2}\right) \cdots \delta \bar{A}_{\mu_{n}}\left(p_{n}\right)}\right|_{\bar{A}=0}
$$

No que segue, vamos calcular explicitamente estes termos.

\subsubsection{Cálculo de $\mathcal{F}(x, k)$}

O primeiro termo da função distribuição, $\mathcal{F}^{(0)}$, que é de ordem "zero" em $e$, deve ser obtido diretamente da definição (5.63), tomando a função de Wigner $w_{\mu \nu}(x, k)$ em ordem "zero" no acoplamento. Mas as únicas contribuições de ordem $e$ para $w_{\mu \nu}$ vêm de $G_{\mu}^{( \pm)}$através dos operadores de $U^{(\bar{A})}$, que na 
aproximação até ordem $e$ são dados apenas por

$$
\begin{aligned}
& U^{(\bar{A})}\left(x, x_{ \pm}\right) \simeq 1 \mp \frac{i e}{\hbar c} \int_{0}^{1} \mathrm{~d} s \frac{y}{2} \cdot \bar{A}\left(x \pm(1-s) \frac{y}{2}\right) \\
& U^{(\bar{A})}\left(x_{ \pm}, x\right) \simeq 1 \pm \frac{i e}{\hbar c} \int_{0}^{1} \mathrm{~d} s \frac{y}{2} \cdot \bar{A}\left(x \pm s \frac{y}{2}\right)
\end{aligned}
$$

Portanto, em ordem "zero" em $e$, devemos tomar $U^{(\bar{A})} \simeq 1$, de maneira que $\mathcal{F}^{(0)}$ pode ser escrito como

$$
\mathcal{F}^{(0)}(x, k)=\eta^{\mu \nu}\left\langle w_{\mu \nu}^{(0)}(x, k)\right\rangle=\int \frac{\mathrm{d}^{4} y}{(2 \pi)^{4}} e^{-i y \cdot k}\left\langle a_{\mu}\left(x_{+}\right) \star a^{\mu}\left(x_{-}\right)\right\rangle .
$$

O cálculo de $\mathcal{F}^{(0)}$ pode ser feito sem dificuldades. Os detalhes estão no Apêndice F.1. Vamos precisar da solução de ondas planas para o campo quântico $a_{\mu}(x)$,

$$
a^{\mu}(x)=\sum_{s_{1}} \int \frac{\mathrm{d}^{3} k_{1}}{(2 \pi)^{3} 2 k_{1}^{0}} \epsilon_{1}^{\mu}\left(a_{1} \mathrm{e}^{-i k_{1} \cdot x}+a_{1}^{\dagger} \mathrm{e}^{i k_{1} \cdot x}\right),
$$

onde $a_{1} \equiv a\left(k_{1}, s_{1}\right)$ e $a_{1}^{\dagger} \equiv a^{\dagger}\left(k_{1}, s_{1}\right)$. Eles possuem "médias térmicas" dadas por ${ }^{4}$

$$
\begin{aligned}
& \left\langle a_{1} a_{2}^{\dagger}\right\rangle=(2 \pi)^{3} 2 k_{1}^{0} \delta_{s_{1} s_{2}} \delta^{3}\left(k_{1}-k_{2}\right)\left(1+n_{B}\left(\left|k_{1}^{0}\right|\right)\right) \\
& \left\langle a_{1}^{\dagger} a_{2}\right\rangle=(2 \pi)^{3} 2 k_{1}^{0} \delta_{s_{1} s_{2}} \delta^{3}\left(k_{1}-k_{2}\right) n_{B}\left(\left|k_{1}^{0}\right|\right)
\end{aligned}
$$

e satisfazem a relação de comutação usual

$$
\left[a_{1}, a_{2}^{\dagger}\right]=(2 \pi)^{3} 2 k_{1}^{0} \delta_{s_{1} s_{2}} \delta^{3}\left(k_{1}-k_{2}\right)
$$

O vetor de polarização $\epsilon_{1}^{\mu} \equiv \epsilon_{1}^{\mu}\left(k_{1}, s_{1}\right)$ é real e transversal ao vetor de onda $k_{1}^{\mu}$, satisfazendo

$$
k_{1} \cdot \epsilon_{1}\left(k_{1}, s_{1}\right)=0 \quad \sum_{s_{1}=1,2} \epsilon_{1 \mu}\left(k_{1}, s_{1}\right) \epsilon_{1}^{\mu}\left(k_{1}, s_{1}\right)=-2
$$

Estas são as principais propriedades que precisamos para obter

$$
\mathcal{F}^{(0)}(x, k)=-\frac{4}{(2 \pi)^{3}} n_{B}\left(\left|k^{0}\right|\right) \theta\left(k^{0}\right) \delta\left(k^{2}\right)
$$

Tendo sido obtida a expressão para $\mathcal{F}^{(0)}$, vamos agora obter a expressão para $\mathcal{F}^{(1)}$, que deve vir dos termos de ordem $e$ na equação de transporte (5.64) quando substituída nela a expansão (5.65). Obtemos

\footnotetext{
${ }^{4}$ Ver pags. $124-125$ de [1].
} 
assim

$$
\begin{aligned}
k^{\mu}\left(\partial_{\mu}-\right. & \left.i e\left[\bar{A}_{\mu},\right]_{M B}\right)\left(\mathcal{F}^{(0)}+\mathcal{F}^{(1)}\right) \\
= & \frac{e}{2} \frac{\partial}{\partial k_{\sigma}} k^{\rho}\left[\bar{f}_{\rho \sigma} \star \mathcal{F}^{(0)}+\mathcal{F}^{(0)} \star \bar{f}_{\rho \sigma}-2 \int \frac{\mathrm{d}^{4} y}{(2 \pi)^{4}} \mathrm{e}^{-i y \cdot k}\left\langle a_{\mu}\left(x_{+}\right) \star \bar{f}_{\rho \sigma} \star a^{\mu}\left(x_{-}\right)\right\rangle\right],
\end{aligned}
$$

onde $\bar{f}_{\rho \sigma}$ é a parte de $\bar{F}_{\rho \sigma}$ independente de $e$, ou seja

$$
\bar{f}_{\rho \sigma}(x)=\partial_{\rho} \bar{A}_{\sigma}(x)-\partial_{\sigma} \bar{A}_{\rho}(x) .
$$

Note que o lado esquerdo vai dar apenas uma contribuição de ordem $e$, vinda de $k \cdot \partial \mathcal{F}^{(1)}$, pois $-i e\left[\bar{A}_{\mu}, \mathcal{F}^{(0)}\right]_{M B}=$ 0 , já que $\mathcal{F}^{(0)}$ não depende das coordenadas. Desta forma, a equação de transporte para $\mathcal{F}^{(1)}$ é dada por

$$
k \cdot \partial \mathcal{F}^{(1)}(x, k)=\frac{e}{2} \frac{\partial}{\partial k_{\sigma}} k^{\rho}\left[2 \bar{f}_{\rho \sigma} \mathcal{F}^{(0)}-2 \int \frac{\mathrm{d}^{4} y}{(2 \pi)^{4}} \mathrm{e}^{-i y \cdot k}\left\langle a_{\mu}\left(x_{+}\right) \star \bar{f}_{\rho \sigma} \star a^{\mu}\left(x_{-}\right)\right\rangle\right] .
$$

Precisamos calcular apenas a integral do lado direito, pois $\mathcal{F}^{(0)}$ já foi calculado. O cálculo de $\mathcal{F}^{(1)}$ está feito no Apêndice F.2. O resultado que obtemos é:

$$
\mathcal{F}^{(1)}(x, k)=-\frac{4 e}{(2 \pi)^{3}} \frac{1}{k \cdot \partial} \frac{\partial}{\partial k_{\sigma}} k^{\rho}\left[n_{B}\left(\left|k^{0}\right|\right) \theta\left(k^{0}\right) \delta\left(k^{2}\right)\left(\bar{f}_{\rho \sigma}(x)-\bar{f}_{\rho \sigma}(x+\theta k)\right)\right] .
$$

O cálculo de $\mathcal{F}^{(2)}$, que é de ordem $e^{2}$, deve vir da seguinte expressão para a equação de transporte (5.64)

$$
\begin{aligned}
& k^{\mu}\left(\partial_{\mu}-i e\left[\bar{A}_{\mu},\right]\right)\left(\mathcal{F}^{(1)}+\mathcal{F}^{(2)}\right) \\
&=\frac{e}{2} \frac{\partial}{\partial k_{\sigma}} k^{\rho}\left[\bar{f}_{\rho \sigma}(x) \star \mathcal{F}^{(1)}(x, k)+\mathcal{F}^{(1)}(x, k) \star \bar{f}_{\rho \sigma}(x)\right. \\
&-i e\left[\bar{A}_{\rho}(x), \bar{A}_{\sigma}(x)\right]_{\mathrm{MB}} \star \mathcal{F}^{(0)}-i e \mathcal{F}^{(0)} \star\left[\bar{A}_{\rho}(x), \bar{A}_{\sigma}(x)\right]_{\mathrm{MB}} \\
&-2 \int \frac{\mathrm{d}^{4} y}{(2 \pi)^{4}} \mathrm{e}^{-i y \cdot k}\left\langle G_{\mu}\left(x_{+}\right) \star\left(-i e\left[\bar{A}_{\rho}, \bar{A}_{\sigma}\right]_{\mathrm{MB}}\right) \star G^{\mu}\left(x_{-}\right)\right\rangle \\
&\left.-2 \int \frac{\mathrm{d}^{4} y}{(2 \pi)^{4}} \mathrm{e}^{-i y \cdot k}\left\langle G_{\mu}\left(x_{+}\right) \star \bar{f}_{\rho \sigma}(x) \star G^{\mu}\left(x_{-}\right)\right\rangle\right] .
\end{aligned}
$$

As contribuições da primeira e segunda linhas do lado direito da igualdade são explicitamente de ordem $e^{2}$, lembrando que $\mathcal{F}^{(1)}$ é de ordem $e$, e são fáceis de serem calculadas, pois $\mathcal{F}^{(0)}$ e $\mathcal{F}^{(1)}$ já estão determinados. A terceira linha também é fácil de ser calculada, visto que o termo já é de ordem $e^{2}$, portanto $G_{\mu}\left(x_{ \pm}\right)=a_{\mu}\left(x_{ \pm}\right)$nesta ordem, e assim podemos usar diretamente o resultado da integral (F.11), simples- 
mente trocando $\bar{f}_{\rho \sigma} \rightarrow-i e\left[\bar{A}_{\rho}, \bar{A}_{\sigma}\right]_{\mathrm{MB}}$, de maneira que obtemos para a integral da terceira linha

$$
\int \frac{\mathrm{d}^{4} y}{(2 \pi)^{4}} \mathrm{e}^{-i y \cdot k}\left\langle a_{\mu}\left(x_{+}\right) \star\left(-i e\left[\bar{A}_{\rho}(x), \bar{A}_{\sigma}(x)\right]_{\mathrm{MB}}\right) \star a^{\mu}\left(x_{-}\right)\right\rangle=-i e \mathcal{F}^{(0)}\left[\bar{A}_{\rho}(x+\theta k), \bar{A}_{\sigma}(x+\theta k)\right]_{\mathrm{MB}} .
$$

O cálculo da contribuição vinda da última linha é um pouco mais elaborado, mas pode ser feito sem grandes dificuldades. Note que ela parece ser de ordem $e$, mas lembre-se que existe ainda uma possível contribuição de ordem $e$ vinda de $G^{\mu}$, e olhando para sua forma em (5.60), esta contribuição poderá vir tanto dos $U^{(\bar{A})}$ 's quanto de $a_{\mu}$, que neste caso será a solução quântica covariante, ou seja, a solução de ondas planas que se transforma covariantemente, conforme discutido no Apêndice G.

Em primeiro lugar note que as possíveis contribuições vindas dos $U^{(\bar{A})}$ 's, quando vistos na forma expandida (5.69), serão proporcionais a $y$, mas visto no espaço dos momentos, $y$ corresponde a uma derivação com relação ao momento $k$,

$$
y^{\mu} \rightarrow i \hbar \frac{\partial}{\partial k_{\mu}}
$$

e portanto dará uma contribuição de $k$ no denominador, portanto subdominante na aproximação de altas temperaturas $p<<k$. As únicas contribuições virão, portanto, de $a_{\mu}$ covariante, dado por

$$
a^{\mu}(x)=\sum_{s_{1}} \int \frac{\mathrm{d}^{3} k_{1}}{(2 \pi)^{3} 2 k_{1}^{0}} \epsilon_{1}^{\mu}\left(a_{1} \mathrm{e}^{-i k_{1} \cdot X}+a_{1}^{\dagger} \mathrm{e}^{i k_{1} \cdot X}\right)
$$

onde $X^{\mu}=x^{\mu}+e \theta^{\mu \nu} \tilde{A}_{\nu}(x)$ é a coordenada covariante e $\tilde{A}_{\nu}(x)=\bar{A}_{\nu}(x)+\frac{1}{k \cdot \bar{D}} \bar{F}_{\nu \alpha}(x) k^{\alpha}$. Os detalhes dos cálculos estão no Apêndice F.3. A expressão final para $\mathcal{F}^{(2)}$ é

$$
\begin{aligned}
\mathcal{F}^{(2)}(x, k)= & i e \frac{1}{k \cdot \partial}\left[k \cdot \bar{A}(x), \mathcal{F}^{(1)}(x, k)\right]_{\mathrm{MB}} \\
& -\frac{4 i e^{2}}{(2 \pi)^{3}} \frac{1}{k \cdot \partial} \frac{\partial}{\partial k_{\sigma}} k^{\rho} \delta\left(k^{2}\right) n_{B}\left(\left|k^{0}\right|\right)\left\{\left[\bar{A}_{\rho}(x), \bar{A}_{\sigma}(x)\right]_{\mathrm{MB}}-\left[\bar{A}_{\rho}(x+\theta k), \bar{A}_{\sigma}(x+\theta k)\right]_{\mathrm{MB}}\right. \\
& \left.+\left[\frac{1}{k \cdot \partial} k \cdot(\bar{A}(x)-\bar{A}(x+\theta k)), \bar{f}_{\rho \sigma}(x+\theta k)\right]_{\mathrm{MB}}\right\}
\end{aligned}
$$

de maneira que já podemos calcular as amplitudes de 2 e 3-pontos em 1-loop.

\subsubsection{Amplitude de 2-pontos}

Para o cálculo da amplitude de 2-pontos, precisamos obter a expressão para a corrente (5.66) até a ordem $e^{2}$. Usando a definição (5.66), em primeira ordem em $e$ a corrente será

$$
\begin{aligned}
J_{\mu}^{(1)}(x) & =-e \int \mathrm{d}^{4} k k_{\mu}\left[\mathcal{F}^{(0)}(x, k)-\mathcal{F}^{(0)}(x,-k)\right] \\
& =0
\end{aligned}
$$


como esperado. Para a ordem seguinte temos

$$
\begin{aligned}
J_{\mu}^{(2)}(x)= & -e \int \mathrm{d}^{4} k k_{\mu}\left[\mathcal{F}^{(1)}(x, k)-\mathcal{F}^{(1)}(x,-k)\right] \\
= & \frac{4 e^{2}}{(2 \pi)^{3}} \int \mathrm{d}^{4} k k_{\mu} \frac{1}{k \cdot \partial} \frac{\partial}{\partial k_{\sigma}} k^{\rho}\left[n_{B}\left(\left|k^{0}\right|\right) \theta\left(k^{0}\right) \delta\left(k^{2}\right)\right. \\
& \left.\times\left[\bar{f}_{\rho \sigma}(x)-\bar{f}_{\rho \sigma}(x+\theta k)+\bar{f}_{\rho \sigma}(x)-\bar{f}_{\rho \sigma}(x-\theta k)\right]\right]
\end{aligned}
$$

Escrevendo os campos $\bar{A}_{\rho}(x)$ e $\bar{A}_{\rho}(x+\theta k)$ como transformada de Fourier

$$
\begin{aligned}
\bar{A}_{\sigma}(x) & =\int \mathrm{d}^{4} p \bar{A}_{\sigma}(p) \mathrm{e}^{i p \cdot x}, \\
\bar{A}_{\sigma}(x+\theta k) & =\int \mathrm{d}^{4} p \bar{A}_{\sigma}(p) \mathrm{e}^{i p \cdot(x+\theta k)},
\end{aligned}
$$

e substituindo em (5.86), obtemos

$$
\begin{aligned}
J_{\mu}^{(2)}(x)= & \frac{4 e^{2}}{(2 \pi)^{3}} \int \mathrm{d}^{4} k k_{\mu} \frac{1}{k \cdot p} \frac{\partial}{\partial k_{\sigma}} k^{\rho} n_{B}\left(\left|k^{0}\right|\right) \theta\left(k^{0}\right) \delta\left(k^{2}\right)\left[2 p_{\rho} \bar{A}_{\sigma}(p)-2 p_{\sigma} \bar{A}_{\rho}(p)\right. \\
& \left.-p_{\rho} \bar{A}_{\sigma}(p) \mathrm{e}^{i p \theta k}-p_{\rho} \bar{A}_{\sigma}(p) \mathrm{e}^{-i p \theta k}+p_{\sigma} \bar{A}_{\rho}(p) \mathrm{e}^{i p \theta k}+p_{\sigma} \bar{A}_{\rho}(p) \mathrm{e}^{-i p \theta k}\right] \\
= & \frac{4 e^{2}}{(2 \pi)^{3}} \int \mathrm{d}^{4} k k_{\mu} \frac{1}{k \cdot p} \frac{\partial}{\partial k_{\sigma}} k^{\rho} n_{B}\left(\left|k^{0}\right|\right) \theta\left(k^{0}\right) \delta\left(k^{2}\right)\left[2(1-\cos (p \times k))\left(p_{\rho} \bar{A}_{\sigma}(p)-p_{\sigma} \bar{A}_{\rho}(p)\right)\right] .
\end{aligned}
$$

Integrando por partes obtemos

$$
J_{\mu}^{(2)}(p)=-\frac{8 e^{2}}{(2 \pi)^{3}} \int \mathrm{d}^{4} k n_{B}\left(\left|k^{0}\right|\right) \theta\left(k^{0}\right) \delta\left(k^{2}\right)(1-\cos p \times k)\left[\eta_{\mu \nu}-\frac{p_{\mu} k_{\nu}+k_{\mu} p_{\nu}}{k \cdot p}+\frac{k_{\mu} k_{\nu} p^{2}}{(k \cdot p)^{2}}\right] \bar{A}^{\nu}(p)
$$

Com isto, usando a (5.67), obtemos a função de 2-pontos a altas temperaturas

$$
\begin{aligned}
\Pi_{\mu \nu}(p) & =\left.\frac{\delta J_{\mu}^{(2)}(-p)}{\delta \bar{A}^{\nu}(p)}\right|_{\bar{A}=0} \\
& =\frac{8 e^{2}}{(2 \pi)^{3}} \int \mathrm{d}^{4} k n_{B}\left(\left|k^{0}\right|\right) \theta\left(k^{0}\right) \delta\left(k^{2}\right)(1-\cos p \times k)\left[\eta_{\mu \nu}-\frac{p_{\mu} k_{\nu}+k_{\mu} p_{\nu}}{k \cdot p}+\frac{k_{\mu} k_{\nu} p^{2}}{(k \cdot p)^{2}}\right] \\
& =\frac{4 e^{2}}{(2 \pi)^{3}} \int \mathrm{d}^{3} k \frac{n_{B}(|\vec{k}|)}{|\vec{k}|}(1-\cos p \times k)\left[\eta_{\mu \nu}-\frac{p_{\mu} k_{\nu}+k_{\mu} p_{\nu}}{k \cdot p}+\frac{k_{\mu} k_{\nu} p^{2}}{(k \cdot p)^{2}}\right],
\end{aligned}
$$

que é exatamente igual ao resultado obtido no capítulo 3 pelo método da amplitude de espalhamento frontal, conforme (3.65). 


\subsubsection{Amplitude de 3-pontos}

Para calcularmos a função de três pontos, devemos primeiro obter a corrente até terceira ordem em $e$. Usando (5.84) e a expansão (F.19) para o último termo, a corrente é dada por

$$
\begin{aligned}
J_{\mu}^{(3)}\left(p_{1}\right) & =\frac{16 i e^{3}}{(2 \pi)^{3}} \int \mathrm{d}^{4} p_{2} \mathrm{~d}^{4} p_{3} \mathrm{~d}^{4} k \theta\left(k^{0}\right) \delta\left(k^{2}\right) n_{B}\left(k^{0}\right) \delta\left(p_{1}+p_{2}+p_{3}\right) \sin \left(\frac{p_{1} \times p_{2}}{2}\right) \\
& \times\left[\left(1-\cos \left(p_{3} \times k\right)\right)\left(L_{\mu \sigma}\left(p_{1}, k\right) \frac{k_{\nu}}{p_{1} \cdot k}+L_{\nu \sigma}\left(p_{3}, k\right) \frac{k_{\mu}}{p_{1} \cdot k}\right) \bar{A}^{\nu}\left(p_{2}\right) L^{\lambda \sigma}\left(p_{3}, k\right) \bar{A}_{\lambda}\left(p_{3}\right)\right. \\
& +\left(1-\cos \left(p_{1} \times k\right)\right) \frac{k \cdot \bar{A}\left(p_{3}\right)}{p_{1} \cdot k} L_{\mu \sigma}\left(p_{1}, k\right) L^{\nu \sigma}\left(p_{1}, k\right) \bar{A}_{\nu}\left(p_{2}\right) \\
& \left.+\left(\cos \left(p_{1} \times k\right)-\cos \left(p_{3} \times k\right)\right) \frac{p_{3} \cdot k}{p_{1} \cdot k} \frac{k \cdot \bar{A}\left(p_{2}\right)}{p_{2} \cdot k} L_{\mu \sigma}\left(p_{1}, k\right) L^{\lambda \sigma}\left(p_{3}, k\right) \bar{A}_{\lambda}\left(p_{3}\right)\right]
\end{aligned}
$$

onde

$$
L_{\mu \nu}(p, k)=\eta_{\mu \nu}-\frac{k_{\mu} p_{\nu}}{p \cdot k}
$$

Isto nos fornece a função de 3-pontos

$$
\begin{aligned}
\Gamma_{\mu \nu \lambda}^{g}\left(p_{1}, p_{2}, p_{3}\right)= & \left.\frac{\delta^{2} J_{\mu}^{(3)}\left(-p_{1}\right)}{\delta \bar{A}^{\nu}\left(p_{2}\right) \delta \bar{A}^{\lambda}\left(p_{3}\right)}\right|_{\bar{A}_{\mu}=0} \\
= & \frac{8 i e^{3}}{(2 \pi)^{3}} \sin \left(\frac{p_{1} \times p_{2}}{2}\right) \int \frac{\mathrm{d}^{3} k}{|\vec{k}|} n_{B}(|\vec{k}|) \frac{1}{k \cdot p_{1}}\left[\left[1-\cos \left(k \times p_{1}\right)\right] k_{\lambda} G_{\mu \nu}\left(k ; p_{1}\right)\right. \\
+ & {\left[1-\cos \left(k \times p_{3}\right)\right]\left[k_{\mu} G_{\nu \lambda}\left(k ; p_{3}\right)+k_{\nu} G_{\mu \sigma}\left(k ; p_{1}\right) G_{\lambda}^{\sigma}\left(k ; p_{3}\right)\right] } \\
+ & {\left[\cos \left(k \times p_{1}\right)-\cos \left(k \times p_{3}\right)\right] \frac{k \cdot p_{3}}{k \cdot p_{2}} k_{\nu} G_{\mu \sigma}\left(k ; p_{1}\right) G_{\lambda}^{\sigma}\left(k ; p_{3}\right) } \\
& \left.-\left(p_{2} \leftrightarrow p_{3} ; \nu \leftrightarrow \lambda\right)\right],
\end{aligned}
$$

onde

$$
G_{\mu \nu}(k ; p)=\eta_{\mu \nu}-\frac{k_{\mu} p_{\nu}+k_{\nu} p_{\mu}}{(k \cdot p)}+\frac{p^{2} k_{\mu} k_{\nu}}{(k \cdot p)^{2}}
$$

Com um pouco de manipulação algébrica pode-se mostrar que este resultado é exatamente igual ao resultado obtido pelo método FSA, conforme equação (3.69).

\subsubsection{Força para partículas neutras}

Vamos agora mostrar, pelo menos qualitativamente, que com os resultados anteriores conseguimos obter a forma da força agindo em uma partícula neutra não-comutativa, conforme equação (5.36). Para 
isto, note que a equação de transporte (5.64) pode ser escrita como

$$
\begin{gathered}
k \cdot \bar{D} \mathcal{F}(x, k)=\frac{e}{2} \frac{\partial}{\partial k_{\sigma}} \int \frac{\mathrm{d}^{4} y}{(2 \pi)^{4}} e^{-i y \cdot k}\left[\left\langle\bar{F}_{\rho \sigma} k^{\rho} \star G_{\mu}^{(+)} \star G^{\mu(-)}+G_{\mu}^{(+)} \star G^{\mu(-)} \star \bar{F}_{\rho \sigma} k^{\rho}\right.\right. \\
\left.\left.-2 G_{\mu}^{(+)} \star \bar{F}_{\rho \sigma} \star G^{\mu(-)}\right\rangle\right] \\
=\frac{e}{2} \frac{\partial}{\partial k_{\sigma}} \int \frac{\mathrm{d}^{4} y}{(2 \pi)^{4}} e^{-i y \cdot k}\left[2 \cos \left(\frac{1}{2} \theta^{\alpha \beta}\left(\partial_{\alpha} \bar{F}_{\rho \sigma} k^{\rho}\right)\left(\partial_{\beta}\left\langle G_{\mu}^{(+)} \star G^{\mu(-)}\right\rangle\right)\right)\right. \\
\left.-2\left\langle G_{\mu}^{(+)} \star \bar{F}_{\rho \sigma} \star G^{\mu(-)}\right\rangle\right]
\end{gathered}
$$

onde usamos (5.63), (5.59) e a propriedade (2.45). Olhando para a forma (5.60) de $G_{\mu}^{( \pm)}$, vemos que ela depende essencialmente de $a_{\mu}$ em ordem dominante, pois como já discutimos, os termos $U^{(\bar{A})}$ contribuem somente com termos subdominantes. Também vemos que (5.95) é quadrática em $G$, portanto quadrática em $a_{\mu}$, de forma que o termo contendo a derivada $\partial_{\beta}$ vai contribuir com $\partial_{\beta}\left\langle G_{\mu}^{(+)} \star G^{\mu(-)}\right\rangle \sim-2 i k_{\beta}$, pois $k$ representa o momento do campo quântico $a_{\mu}$ no espaço dos momentos. Além disto, pelos resultados (5.70), (F.11), (5.82) e (F.15) podemos concluir que

$$
\begin{aligned}
& \int \frac{\mathrm{d}^{4} y}{(2 \pi)^{4}} e^{-i y \cdot k}\left\langle G_{\mu}^{(+)} \star G^{\mu(-)}\right\rangle \sim \mathcal{F}^{(0)} \sim n_{B}(|\vec{k}|) \\
& \int \frac{\mathrm{d}^{4} y}{(2 \pi)^{4}} e^{-i y \cdot k}\left\langle G_{\mu}^{(+)} \star \bar{F}_{\rho \sigma} \star G^{\mu(-)}\right\rangle \sim \mathcal{F}^{(0)} \bar{F}_{\rho \sigma} \sim n_{B}(|\vec{k}|) \bar{F}_{\rho \sigma},
\end{aligned}
$$

de forma que (5.95) pode ser escrita como

$$
k \cdot \bar{D} \mathcal{F}(x, k)=-e \frac{\partial}{\partial k_{\sigma}}\left(n_{B}(|\vec{k}|)[1-\cos k \times i \partial] \bar{F}_{\rho \sigma} k^{\rho}\right) .
$$

Comparando a expressão (5.97) com (5.35), vemos que a força neste caso é dada por

$$
X_{\sigma} \sim[1-\cos k \times i \partial] \bar{F}_{\rho \sigma} k^{\rho}
$$

portanto tem a mesma forma de (5.36), o que justifica a forma (5.31) para a força no caso clássico.

\subsubsection{Pressão na NCQED}

Da mesma forma que no caso do campo de gauge da seção 4.2.4, podemos definir um tensor de energia momento para a NCQED em termos da função de Wigner (5.37), dado por

$$
\hat{T}_{\mu \nu}(x)=\left\langle\int \mathrm{d}^{4} k\left[W_{\mu \nu}(x, k)-\frac{1}{4} \eta_{\mu \nu} W_{\lambda}^{\lambda}(x, k)\right]\right\rangle,
$$

onde o traço foi trocado pela média térmica dos campos. Em ordem mais baixa, podemos calcular $W$ facilmente. Fazendo os campos de fundo iguais a zero, $\bar{A}_{\mu}=0$, resta apenas a média térmica dos campos 
quânticos. Usando (5.38), (G.3) e as relações (5.71-5.75), obtemos

$$
\begin{aligned}
\left\langle W_{\mu \nu}^{(0)}(x, k)\right\rangle & =\int \frac{\mathrm{d}^{4} y}{(2 \pi)^{4}} e^{-i y \cdot k}\left\langle\left(\partial_{\mu} a^{\lambda}\left(x_{+}\right)-\partial^{\lambda} a_{\mu}\left(x_{+}\right)\right) \star\left(\partial_{\lambda} a_{\nu}\left(x_{-}\right)-\partial_{\lambda} a_{\nu}\left(x_{-}\right)\right)\right\rangle \\
& =\frac{4}{(2 \pi)^{3}} k_{\mu} k_{\nu} \theta\left(k_{0}\right) \delta\left(k^{2}\right) n_{B}\left(k_{0}\right),
\end{aligned}
$$

onde desprezamos um termo independente da temperatura. O tensor de energia-momento em ordem mais baixa é dado então por

$$
\begin{aligned}
\hat{T}_{\mu \nu}^{(0)}(x) & =\left\langle\int \mathrm{d}^{4} k\left[W_{\mu \nu}^{(0)}(x, k)-\frac{1}{4} \eta_{\mu \nu} W_{\lambda}^{(0) \lambda}(x, k)\right]\right\rangle \\
& =\frac{4}{(2 \pi)^{3}} \int \mathrm{d}^{4} k \theta\left(k_{0}\right) \delta\left(k^{2}\right) n_{B}\left(k_{0}\right)\left(k_{\mu} k_{\nu}-\frac{1}{4} \eta_{\mu \nu} k^{2}\right) \\
& =\frac{4}{(2 \pi)^{3}} \int \mathrm{d}^{4} k \theta\left(k_{0}\right) \delta\left(k^{2}\right) n_{B}\left(k_{0}\right) k_{\mu} k_{\nu},
\end{aligned}
$$

pois $k^{2}=0$. Com isto calculamos a componente temporal do tensor, que corresponde à densidade de energia do campo

$$
\begin{aligned}
E \equiv \hat{T}_{00}^{(0)}(x) & =\frac{4}{(2 \pi)^{3}} \int \mathrm{d}^{4} k \theta\left(k_{0}\right) \delta\left(k^{2}\right) n_{B}\left(k_{0}\right) k_{0} k_{0} \\
& =\frac{2}{(2 \pi)^{3}} \int \mathrm{d}^{3} k \frac{|\vec{k}|}{\mathrm{e}^{|\vec{k}| / T}-1} \\
& =\frac{8 \pi}{(2 \pi)^{3}} \int_{0}^{\infty} \mathrm{d} k \frac{k^{3}}{\mathrm{e}^{k / T}-1} \\
& =\frac{\pi^{2}}{15} T^{4} .
\end{aligned}
$$

Para um gás relativístico, como vimos em (1.91), a pressão é dada por $E / 3$, de forma que

$$
P^{(0)}=\frac{1}{3} E=\frac{\pi^{2}}{45} T^{4}
$$

exatamente os mesmos resultados obtidos em (1.88) e (1.90) para o gás de fótons livres.

Como sabemos da seção 3.8, a próxima correção à pressão de um gás de fótons não-comutativos é de ordem $e^{2}$, conforme expressão (3.71) para a energia livre de Helmholtz. Portanto, devemos olhar para o tensor de energia-momento em segunda ordem em $e$. A função de Wigner em segunda ordem é

$$
\left\langle W_{\mu \nu}^{(2)}(x, k)\right\rangle=\int \frac{\mathrm{d}^{4} y}{(2 \pi)^{4}} e^{-i y \cdot k}(-i e)^{2}\left\langle\left[a_{\mu}\left(x_{+}\right), a^{\lambda}\left(x_{+}\right)\right]_{\mathrm{MB}} \star\left[a_{\lambda}\left(x_{-}\right), a_{\nu}\left(x_{-}\right)\right]_{\mathrm{MB}}\right\rangle .
$$

O cálculo desta expressão é bastante trabalhoso, mas pode ser feito sem dificuldades. Isto foi feito, e o resul- 
tado é que, quando colocado no tensor de energia-momento, fornece exatamente o resultado para a pressão em segunda ordem vinda de (3.71). É fácil entender a estrutura desta expressão. Ela contém o produto de quatro campos quânticos, de forma que, pelas relações (5.71)-(5.75), teremos no resultado final o produto de duas funções de Bose, $n_{B}$, exatamente conforme encontrado na expressão (3.71). Além disso, cada comutador Moyal presente na expressão (5.104) dará origem a uma função seno envolvendo o parâmetro não-comutativo, portanto o resultado final ser proporcional a seno ao quadrado. O que é interessante é que a expressão obtida é exatamente igual, o que não é óbvio, pois outras contribuições proporcionais a $e^{2}$ devem vir da quantização do campo $a_{\mu}$. Estes termos dariam contribuição à outra parte da função de Wigner $W^{(2)}$, por exemplo na parte contendo $(\partial A-\partial A)^{2}$, como em (5.100). Mas estas contribuições devem se cancelar de maneira não-trivial, pois o cálculo de (5.104) já fornece o resultado correto. Este cancelamento exato da parte vinda da covariantização do campo quântico deve ter um significado mais profundo que precisa ser melhor estudado.

Os principais resultados apresentados nesta seção, equações (5.59)-(5.64), assim como a quantização do campo quântico pelo método do campo de fundo apresentado no Apêndice G, foram publicados em [71]. 



\section{Conclusão}

Neste trabalho abordamos dois tópicos de grande interesse na teoria de campos atualmente. O primeiro foi a teoria de campos a temperatura finita, generalização direta da teoria de campos usual, necessária para incluir efeitos de altas temperaturas e densidades no estudo de sistemas físicos, visto que são muitos os cenários do universo onde estas características se apresentam. O exemplo mais próximo do nosso cotidiano são os plasmas produzidos em laboratório, indo mais longe temos interiores de estrelas, núcleos galácticos e fenômenos intrínsecos ao universo primordial. O outro tópico abordado neste trabalho foi o estudo de teorias definidas em espaços não-comutativos, visto que um dos limites de baixa energia da teoria de cordas pode ser descrito efetivamente por estas teorias não-comutativas. Na falta de uma teoria que descreva de maneira correta as interações em escalas de energia muito alta, ou distâncias muito pequenas, que é quando efeitos quânticos e gravitacionais se misturam, temos de utilizar teorias que, pelo menos efetivamente, possam responder a perguntas que o modelo padrão não descreva satisfatoriamente. A teoria não-comutativa nasceu da tentativa de se entender as divergências que cercam a QED e a QCD. Neste trabalho tentamos dar uma idéia de como tratar sistemas onde estes dois tópicos aparecem juntos. Para isso tomamos como referência a eletrodinâmica quântica usual, que é uma teoria muito bem fundamentada e bem estabelecida já há quase um século.

O método dos diagramas de Feynman para tratar da QED, tanto a temperatura zero quanto a temperatura finita, já foi extensivamente utilizado, obtendo-se resultados muito bons. No tratamento de sistemas a temperatura finita os cálculos são bastante complicados devido à necessidade de se fazer somatórias sobre freqüências, e na maioria das vezes estes cálculos não são óbvios. Além disso, às vezes diagramas de ordens diferentes dão contribuições de mesma ordem, portanto deve-se adotar um procedimento de "ressomação", de forma a se considerar todos os possíveis diagramas que contribuem para um dado termo, e isto pode tornar o cálculo muito complicado. Entretanto na maioria das vezes o limite de altas temperaturas de um sistema pode ser visto como uma média estatística de um sistema em equilíbrio térmico, e então outros métodos podem ser utilizados. Sem dúvida o método de Feynman é muito eficaz, porém muito complicado em alguns casos. Desta forma, dependendo da quantidade física que estamos querendo calcular, ou dependendo do limite em que o cálculo deve ser feito, podemos utilizar algum outro método que forneça exatamente os mesmos resultados. Esta foi exatamente uma das propostas do nosso projeto: mostrar que o método das equações de transporte clássicas de Boltzmann pode ser aplicado à QED não-comutativa a altas temperaturas. De fato é intuitivo pensar que um plasma a altas temperaturas deva se comportar, na média, como um conjunto de partículas clássicas em equilíbrio térmico. 
No primeiro capítulo fizemos uma breve exposição da teoria quântica de campos, tanto a temperatura nula quanto a temperatura finita. No segundo capítulo discutimos uma generalização para campos nãouniformes ao problema de um elétron movendo-se em um plano, sujeito a um campo magnético intenso, conhecido como problema de Landau, onde a não-comutatividade das coordenadas aparece naturalmente. Apresentamos em seguida a formulação não-comutativa da QED a temperatura nula. No terceiro capítulo estudamos a formulação da QED em espaços não-comutativos a temperatura finita, e apresentamos os resultados obtidos para o cálculo das amplitudes de 2, 3 e 4-pontos no limite estático a altas temperaturas pelo método de Feynman. Mostramos que as amplitudes com índices puramente espaciais estão relacionadas por meio de identidades de Ward, de forma que a ação efetiva no limite estático a altas temperaturas pôde ser obtida. No quarto capítulo fizemos uma breve revisão da teoria das equações de transporte, método que é muito útil para o cálculo de propriedades físicas de sistemas em equilíbrio térmico a altas temperaturas. Aplicamos este método ao caso da QED não-comutativa a altas temperaturas e mostramos que os mesmos resultados em 1-loop do terceiro capítulo podem ser obtidos de maneira muito mais direta. Uma característica comum aos dois métodos é a presença da não-comutatividade, que torna os cálculos extremamente complexos. Os cálculos realizados utilizando-se as equações de transporte dependem basicamente do conhecimento das forças que agem na partícula, ou seja, das suas equações dinâmicas. Para uma partícula carregada, mesmo no espaço não-comutativo, é intuitivo propor uma forma para a força conforme a eq. (5.15), devido ao comportamento parecido com o de dipolos da estrutura da teoria. Já para uma partícula neutra a forma desta força não é trivial, e diante disso tornou-se necessária uma formulação que pudesse dar uma idéia de como se justificar a forma da força (5.31) a ser utilizada nas equações de transporte clássicas. O método de Wigner, utilizado para tratar sistemas quânticos pelas equações de transporte, forneceu a resposta que queríamos. Em analogia com o tratamento existente para a QCD, desenvolvido por Elze, Heinz e colaboradores, foi possível propor uma equação de transporte a ser satisfeita pelos fótons não-comutativos, e então a forma da força pôde ser derivada naturalmente. Isto mostra que este método pode ser muito útil quando não temos nenhuma informação sobre a força que age no sistema.

Paralelo a esta linha principal do projeto, que era demonstrar, em altas temperaturas, a equivalência dos métodos de equações de transporte com o método tradicional de Feynman, desenvolvemos vários outros tópicos relacionados. Com respeito a efeitos de temperatura finita, estudamos as massas de blindagem dos campos elétrico e magnético devido a efeitos puramente não-comutativos, e vimos que uma correção à massa elétrica deve existir devido à presença da não-comutatividade, embora ela se desenvolva apenas acima de uma temperatura muito alta, o que caracteriza uma transição de fases de primeira ordem. Já para a parte magnética não há nenhuma contribuição. Outro resultado interessante é que, a altíssimas temperaturas, o tensor de auto-energia do fóton não depende do parâmetro de não-comutatividade, conforme eq. (3.17), e a contribuição é da mesma ordem que aquela vinda da QED comutativa a temperatura finita.

Com relação ao aspecto da não-comutatividade, o principal resultado que obtivemos foi a quantização correta do campo de gauge $a_{\mu}$ pelo método do campo de fundo, no limite em que o campo de fundo varia fracamente com relação ao campo quântico. Isto se fez necessário quando se percebeu que faltavam termos de ordem $e^{3}$ para que as contribuições às amplitudes de 3-pontos em 1-loop fornecessem o resultado 
correto. O único lugar de onde estes termos poderiam vir era do próprio campo quântico, que no caso geral apresenta uma estrutura bem complexa, dependente do campo de fundo $\bar{A}_{\mu}$. Levar em conta de forma correta estas contribuições é, sem dúvida, a parte mais trabalhosa, mas isto é inerente à não-comutatividade, e não ao método das equações de transporte. Por último citamos a tentativa em se obter a pressão de um gás de fótons não-comutativos através do tensor de energia-momento. Como foi discutido na última seção do capítulo 5, o primeiro termo que contribui à pressão é fácil de se calcular, depende basicamente do conhecimento da função distribuição em ordem mais baixa, e não tem dependência com o parâmetro de não-comutatividade. Já para a primeira correção não-nula os cálculos são mais trabalhosos, e ainda assim não foi demonstrado porque as outras possíveis correções vindas do campo quântico se cancelam exatamente. Deve haver um motivo mais geral para explicar porque estas contribuições se cancelam, mas este é um assunto que precisa ser melhor estudado.

Um dos interesses no cálculo de correções à pressão de um gás de férmions ou bósons vem da astrofísica. Sabemos que o produto final da evolução de uma estrela pode ser uma anã branca, uma estrela de nêutrons ou um buraco negro, dependendo basicamente de sua massa inicial. Em uma anã branca, o colapso gravitacional é contrabalançado pela pressão de degenerescência dos elétrons, enquanto que em uma estrela de nêutrons é a pressão dos nêutrons que impede que ela colapse para um buraco negro. A determinação correta do limite de massa para o qual uma estrela vai evoluir para o colapso é uma questão importante em astrofísica, e é conhecido como limite de Chandrasekhar, dado por $M \simeq 1,4 M_{S}$ onde $M_{S}$ corresponde a uma massa solar. Anãs brancas com massa maior que o limite de Chandrasekhar não podem existir, e uma maneira de se entender este limite é considerar que os elétrons estão a uma densidade tão alta que eles se tornam relativísticos, aí podemos usar os resultados da teoria de campos a temperatura e densidade finitas. Correções de primeira ordem na pressão de degenerescência dos elétrons devem mudar o valor do limite de Chandrasekhar. Para uma estrela de nêutrons os cálculos são muito mais complicados, pois o equilíbrio se dá entre a pressão dos nêutrons e a pressão gravitacional. Só que um gás de nêutrons relativísticos deve ser tratado por meio da QCD a altas temperaturas e densidade, conforme indicado no diagrama de fases $T \times \mu$ da introdução. Ainda não é claro qual a contribuição devida a correções à pressão de um gás de nêutrons ao limite de Chandrasekhar, no entanto observa-se estrelas de nêutrons com massas que variam de 1, 4 a 1,6 $M_{S}$, portanto um valor maior que o limite anterior, indicando que efeitos da interação nuclear forte devem desempenhar um papel fundamental. Outra aplicação onde cálculos a densidade e temperatura finita são importantes é na perda de energia por estrelas devido a emissão de partículas fracamente interagentes, como os neutrinos por exemplo. Esta é uma questão muito importante na astrofísica. Os principais sistemas onde esses processos ocorrem são nos núcleos de supernovas do tipo II, com temperaturas da ordem de $50 \mathrm{MeV}$ e potencial químico da ordem de $350 \mathrm{MeV}$. Também podem ocorrer em núcleos de estrelas gigantes vermelhas ou anãs brancas. O processo dominante que envolve a emissão de neutrinos em estrelas é chamado "decaimento plasmon", que nada mais é que o decaimento dos fótons transversais e longitudinais em pares de neutrinos. Com relação a efeitos da não-comutatividade, como vimos, a altas temperaturas seus efeitos podem ser mais notáveis, pois a presença comum de um termo do tipo $\theta T$ mostra que a altíssimas temperaturas a não-comutatividade pode se tornar mais evidente em 
determinados processos. A época da inflação do universo é, sem dúvida, um dos melhores "laboratórios" para se testar seus efeitos.

Estas e outras questões ainda em aberto da astrofísica e da cosmologia necessitam, sem dúvida, de um tratamento da teoria de campos a temperatura finita, e muitas vezes cálculos por meio de equações de transporte podem fornecer resultados tão bons quanto os obtidos pelo método de Feynman, como demonstramos aqui.

Os principais resultados apresentados e discutidos nesta tese foram publicados, conforme referências $[70,71,72,73]$. 


\section{Apêndice A}

\section{A.1 Revisão de conceitos de mecânica}

Seja uma lagrangeana $L(q, \dot{q})$, função das coordenadas generalizadas $q_{i}(t)$ e das velocidades correspondentes $\dot{q}_{i}(t)$. As equações de movimento de Euler-Lagrange deste sistema são dadas por

$$
\frac{\partial L}{\partial q_{i}(t)}-\frac{\mathrm{d}}{\mathrm{d} t} \frac{\partial L}{\partial \dot{q}_{i}(t)}=0 .
$$

Os momentos canonicamente conjugados de $q^{i}$ são obtidos por

$$
p_{i}=\frac{\partial L}{\partial \dot{q}_{i}}
$$

e a hamiltoniana do sistema pode ser construída por meio de uma transformação de Legendre

$$
H(q, p)=\sum_{i} p_{i} \dot{q}_{i}(p, q)-L[q, \dot{q}(p, q)]
$$

onde a soma é sobre todas as coordenadas e momentos do sistema.

As equações de Hamilton que seguem da hamiltoniana (A.3) são

$$
\dot{q}_{i}=\frac{\partial H}{\partial p_{i}}, \quad \dot{p}_{i}=-\frac{\partial H}{\partial q_{i}} .
$$

O parênteses de Poisson ou comutador de Poisson de duas quantidades $f, g$ é definido por

$$
\{f, g\}=\sum_{i}\left(\frac{\partial f}{\partial q_{i}} \frac{\partial g}{\partial p_{i}}-\frac{\partial f}{\partial p_{i}} \frac{\partial g}{\partial q_{i}}\right)
$$

e seguem diretamente as propriedades

$$
\left\{q_{i}, q_{j}\right\}=0=\left\{p_{i}, p_{j}\right\}, \quad\left\{q_{i}, p_{j}\right\}=\delta_{i j} .
$$




\section{Apêndice B}

\section{B.1 Algumas propriedades do teorema dos resíduos}

Para o cálculo de alguns somatórios algumas vezes é mais conveniente usarmos integrais no plano complexo e aplicarmos algumas propriedades do teorema dos resíduos. As propriedades abaixo podem ser encontradas nas págs. 84-86 da ref. [66]:

1. Seja $g(z)$ uma função analítica no interior de um contorno fechado e sobre $C$, exceto em um número finito de singularidades isoladas em $z=a_{1}, a_{2}, \cdots, a_{n}$, todas situadas no interior de $C$. Então

$$
\oint_{C} g(z) \mathrm{d} z=2 \pi i \sum_{j=1}^{n} \operatorname{Res}\left[g\left(a_{j}\right)\right],
$$

onde $\operatorname{Res}\left[g\left(a_{j}\right)\right]$ é o resíduo de $g$ no ponto $a_{j}$. O caminho $C$ está orientado no sentido anti-horário.

2. Para uma singularidade (ou pólo) de ordem $m$ em $z=a$, vale a fórmula seguinte para calcular o resíduo:

$$
\operatorname{Res}[g(a)]=\frac{1}{(m-1) !} \lim _{z \rightarrow a} \frac{\mathrm{d}^{m-1}}{\mathrm{~d} z^{m-1}}\left[(z-a)^{m} g(z)\right]
$$

3. Outra maneira de calcular o resíduo é a seguinte: Se $g$ for da forma

$$
g(z)=\frac{\varphi(z)}{\psi(z)}
$$

tal que $\psi(z)$ tem um pólo simples em $z=a$ e $\varphi(a) \neq 0$, então:

$$
\operatorname{Res}[g(a)]=\frac{\varphi(a)}{\psi^{\prime}(a)}
$$




\section{B.2 Soma de frequiências para bósons}

Queremos mostrar a seguinte relação:

$$
\begin{aligned}
T \sum_{n=-\infty}^{\infty} f\left(k_{0}=i \omega_{n}\right)= & \frac{1}{2 \pi i} \int_{-i \infty}^{+i \infty} \mathrm{d} k_{0} \frac{1}{2}\left[f\left(k_{0}\right)+f\left(-k_{0}\right)\right] \\
& +\frac{1}{2 \pi i} \int_{-i \infty+\epsilon}^{+i \infty+\epsilon} \mathrm{d} k_{0}\left[f\left(k_{0}\right)+f\left(-k_{0}\right)\right] \frac{1}{\mathrm{e}^{\beta k_{0}}-1},
\end{aligned}
$$

onde $f\left(k_{0}\right)$ é uma função que não possui singularidades ao longo do eixo imaginário de $k_{0}$ e $\omega_{n}=\frac{2 \pi n}{\beta}$, onde $\beta=1 / T$.

Vamos começar mostrando a seguinte relação:

$$
T \sum_{n=-\infty}^{\infty} f\left(k_{0}=i \omega_{n}\right)=\frac{T}{2 \pi i} \oint_{C} \mathrm{~d} k_{0} f\left(k_{0}\right) \frac{1}{2} \beta \operatorname{coth}\left(\frac{1}{2} \beta k_{0}\right),
$$

sendo o contorno $C$ no plano complexo de $k_{0}$ da forma indicada na Figura B.1.

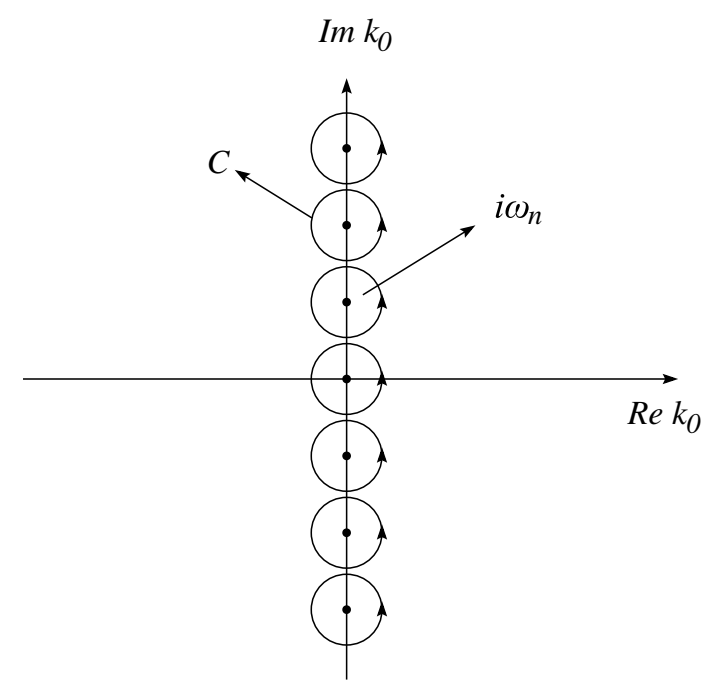

Figura B.1: Contorno de integração da equação (B.6).

Primeiro note que a função cotangente hiperbólico pode ser escrita de diversas formas:

$$
\frac{1}{2} \beta \operatorname{coth}\left(\frac{1}{2} \beta k_{0}\right)=\frac{1}{2} \beta\left(\frac{1+\mathrm{e}^{-\beta k_{0}}}{1-\mathrm{e}^{-\beta k_{0}}}\right)=\frac{1}{2} \beta\left(-1-\frac{2}{\mathrm{e}^{-\beta k_{0}}-1}\right)=\frac{1}{2} \beta\left(1+\frac{2}{\mathrm{e}^{\beta k_{0}}-1}\right)
$$

e tem pólos em $k_{0}=\frac{2 \pi i n}{\beta} \equiv a_{n}$ e é analítica e limitada em todos os outros pontos. Aplicando a propriedade (B.1) ao lado direito de (B.6) temos

$$
\frac{T}{2 \pi i} \oint_{C} \mathrm{~d} z f(z) \frac{1}{2} \beta \operatorname{coth}\left(\frac{1}{2} \beta z\right)=T \sum_{n} \operatorname{Res}\left[f\left(a_{n}\right) \frac{1}{2} \beta \operatorname{coth}\left(\frac{1}{2} \beta a_{n}\right)\right] .
$$


Agora os resíduos podem ser calculados usando-se a propriedade (B.4), fazendo

$$
\varphi\left(a_{n}\right)=f\left(a_{n}\right)\left(1+\mathrm{e}^{-\beta a_{n}}\right) \quad, \quad \psi\left(a_{n}\right)=\left(1-\mathrm{e}^{-\beta a_{n}}\right),
$$

onde usamos (B.7), de forma que $\psi^{\prime}\left(a_{n}\right)=\beta \mathrm{e}^{-\beta a_{n}}$, e então

$$
\begin{aligned}
\operatorname{Res}\left[f\left(a_{n}\right) \frac{1}{2} \beta \operatorname{coth}\left(\frac{1}{2} \beta a_{n}\right)\right] & =\frac{\varphi\left(a_{n}\right)}{\psi^{\prime}\left(a_{n}\right)} \\
& =\frac{f\left(\frac{2 \pi i n}{\beta}\right) \frac{1}{2} \beta\left(1+\mathrm{e}^{-2 \pi i n}\right)}{\beta \mathrm{e}^{-2 \pi i n}} \\
& =f\left(\frac{2 \pi i n}{\beta}\right) \\
& =f\left(k_{0}=i \omega_{n}\right)
\end{aligned}
$$

pois $\mathrm{e}^{-2 \pi i n}=1$ para qualquer $n$ inteiro. Substituindo finalmente em (B.8) obtemos a relação que queríamos demonstrar:

$$
\frac{T}{2 \pi i} \oint_{C} \mathrm{~d} k_{0} f\left(k_{0}\right) \frac{1}{2} \beta \operatorname{coth}\left(\frac{1}{2} \beta k_{0}\right)=T \sum_{n=-\infty}^{\infty} f\left(k_{0}=i \omega_{n}\right)
$$

Agora vamos mostrar a relação (B.5). Em primeiro lugar note que o contorno da Figura B.1 pode ser deformado da seguinte forma, representado na Figura B.2.

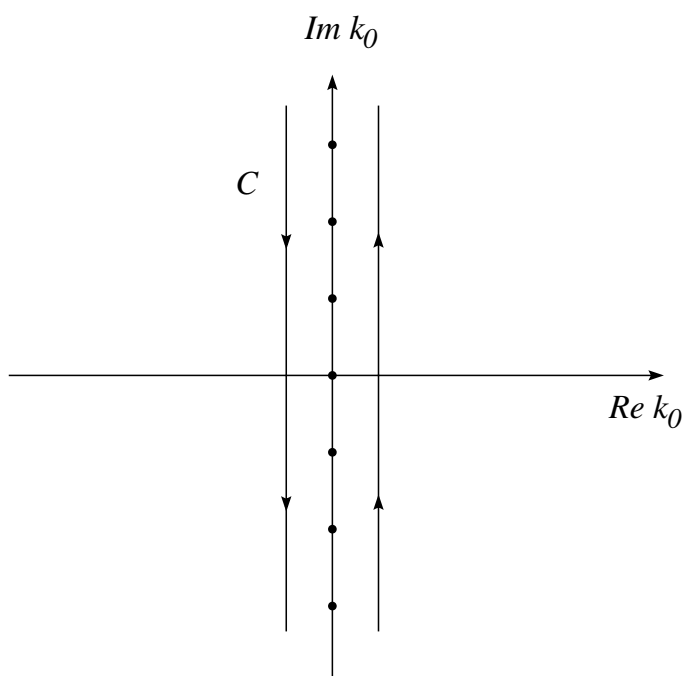

Figura B.2: Contorno de integração equivalente ao da Fig. B.1.

A integral de contorno no lado esquerdo de (B.11) pode ser escrita em termos do contorno da Figura 
B. 2 como

$$
\begin{aligned}
\frac{T}{2 \pi i} \oint_{C} \mathrm{~d} k_{0} f\left(k_{0}\right) \frac{1}{2} \beta \operatorname{coth}\left(\frac{1}{2} \beta k_{0}\right)= & \frac{1}{2 \pi i} \int_{i \infty-\epsilon}^{-i \infty-\epsilon} \mathrm{d} k_{0} f\left(k_{0}\right)\left(-\frac{1}{2}-\frac{1}{\mathrm{e}^{-\beta k_{0}}-1}\right) \\
& +\frac{1}{2 \pi i} \int_{-i \infty+\epsilon}^{+i \infty+\epsilon} \mathrm{d} k_{0} f\left(k_{0}\right)\left(\frac{1}{2}+\frac{1}{\mathrm{e}^{\beta k_{0}}-1}\right)
\end{aligned}
$$

onde usamos (B.7) para reescrever o cotangente hiperbólico de uma forma apropriada. Como o parâmetro $\epsilon$ deve ser tomado como zero no final, não estamos incluindo as integrais nas extremidades superior e inferior do contorno, elas não contribuem. Agora, fazendo $k_{0} \rightarrow-k_{0}$ na primeira integral, podemos escrever tudo em termos do caminho do lado direito da figura, de forma que obtemos finalmente

$$
\begin{aligned}
\frac{T}{2 \pi i} \oint_{C} \mathrm{~d} k_{0} f\left(k_{0}\right) \frac{1}{2} \beta \operatorname{coth}\left(\frac{1}{2} \beta k_{0}\right)= & \frac{1}{2 \pi i} \int_{-i \infty}^{+i \infty} \mathrm{d} k_{0} \frac{1}{2}\left[f\left(k_{0}\right)+f\left(-k_{0}\right)\right] \\
& +\frac{1}{2 \pi i} \int_{-i \infty+\epsilon}^{+i \infty+\epsilon} \mathrm{d} k_{0}\left[f\left(k_{0}\right)+f\left(-k_{0}\right)\right] \frac{1}{\mathrm{e}^{\beta k_{0}}-1},
\end{aligned}
$$

onde fizemos $\epsilon \rightarrow 0$ na primeira integral porque $f\left(k_{0}\right)$ não é singular em nenhum ponto do eixo imaginário, ao contrário da segunda integral, que ainda contém uma dependência singular no denominador. Com isto demonstramos a relação (B.5).

Vamos aplicar os resultados anteriores para um caso específico, que é quando $f$ é da forma

$$
f\left(k_{0}\right)=\frac{-1}{k_{0}^{2}-\omega^{2}}
$$

Substituindo em (B.5), obtemos:

$$
T \sum_{n=-\infty}^{\infty} \frac{-1}{k_{0}^{2}-\omega^{2}}=\frac{1}{2 \pi i} \int_{-i \infty}^{+i \infty} \mathrm{d} k_{0}\left[\frac{-1}{k_{0}^{2}-\omega^{2}}\right]+\frac{1}{2 \pi i} \int_{-i \infty+\epsilon}^{+i \infty+\epsilon} \mathrm{d} k_{0}\left[\frac{-2}{k_{0}^{2}-\omega^{2}}\right] \frac{1}{\mathrm{e}^{\beta k_{0}}-1}
$$

onde $k_{0}=i \omega_{n}=\frac{2 \pi i n}{\beta}$. Na primeira integral fazemos a mudança de variáveis

$$
i k_{0}=k_{4}, \quad \mathrm{~d} k_{0}=-i \mathrm{~d} k_{4}
$$

que corresponde a uma rotação para o eixo imaginário dos momentos. A segunda integral deve ser feita ao longo do caminho $l_{1}$, e uma maneira de fazer isso é usar mais uma vez o teorema dos resíduos. Vamos fechar o caminho $l_{1}$ com um semicírculo $l_{2}$ ao longo do plano do lado direito do eixo $k_{0}$, da forma mostrada na Figura B.3. Desta forma temos um caminho fechado $C=l_{1}+l_{2}$, e vale a propriedade (B.1), escrita 
formalmente como:

$$
\int_{l_{1}}+\int_{l_{2}}=\oint_{C}=-2 \pi i \sum_{j} \operatorname{Res}\left[g\left(a_{j}\right)\right]
$$

O sinal negativo é porque neste caso a curva $C$ está orientada no sentido horário. No entanto, o único pólo que existe no semiplano direito é o ponto $k_{0}=\omega$, de segunda ordem.

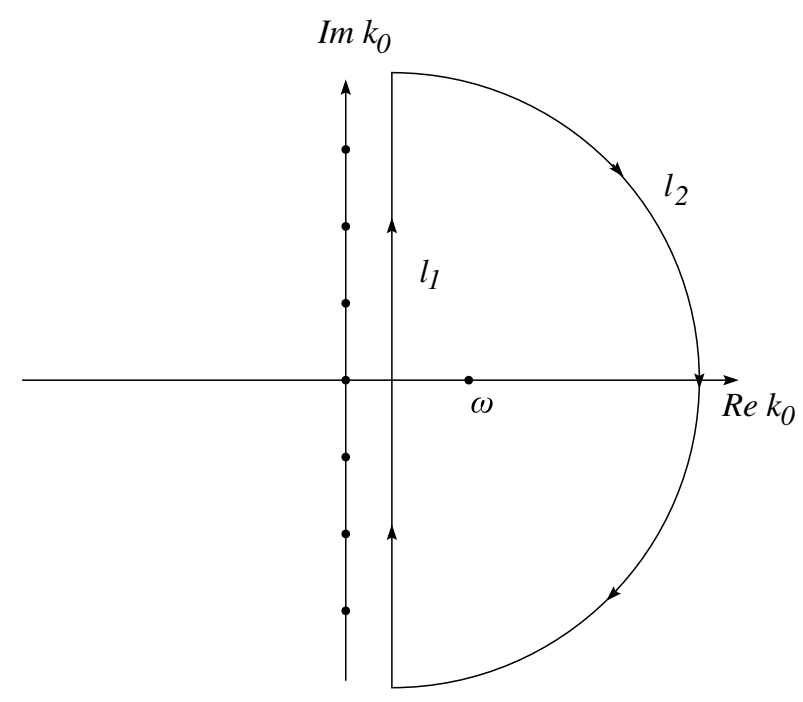

Figura B.3: Contorno $C=l_{1}+l_{2}$.

Estamos interessados na integral ao longo de $l_{1}$. Portanto, tomando

$$
g(z)=\frac{-2}{2 \pi i} \frac{1}{\left(z^{2}-\omega^{2}\right)} \frac{1}{\left(\mathrm{e}^{\beta z}-1\right)},
$$

obtemos

$$
\int_{l_{1}} g(z) \mathrm{d} z=-\int_{l_{2}} g(z) \mathrm{d} z-2 \pi i \operatorname{Res}[g(z=\omega)]
$$

A integral ao longo de $l_{2}$ é nula, pois neste caminho devemos tomar $\left|k_{0}\right| \rightarrow \infty$, portanto a integral se anula devido ao denominador. Ficamos portanto com

$$
\begin{aligned}
\frac{-2}{2 \pi i} \int_{-i \infty+\epsilon}^{+i \infty+\epsilon} \mathrm{d} z \frac{1}{\left(z^{2}-\omega^{2}\right)} \frac{1}{\left(\mathrm{e}^{\beta z}-1\right)} & =-2 \pi i \operatorname{Res}\left[\frac{-2}{2 \pi i} \frac{1}{\left(z^{2}-\omega^{2}\right)} \frac{1}{\left(\mathrm{e}^{\beta z}-1\right)}\right] \\
& =-2 \pi i \lim _{z \rightarrow \omega} \frac{\mathrm{d}}{\mathrm{d} z}\left[(z-\omega)^{2} \frac{-2}{2 \pi i} \frac{1}{\left(z^{2}-\omega^{2}\right)} \frac{1}{\left(\mathrm{e}^{\beta z}-1\right)}\right] \\
& =\frac{1}{\omega} \frac{1}{\mathrm{e}^{\beta \omega}-1},
\end{aligned}
$$

onde usamos a propriedade (B.2) para calcular o resíduo no ponto $z=\omega$. Substituindo este resultado de 
volta na (B.15) e fazendo a mudança de variáveis (B.16) na primeira integral, obtemos finalmente:

$$
T \sum_{n=-\infty}^{\infty} \frac{-1}{k_{0}^{2}-\omega^{2}}=\frac{1}{2 \pi i} \int_{-\infty}^{+\infty} \frac{1}{k_{4}^{2}+\omega^{2}} \mathrm{~d} k_{4}+\frac{1}{\omega} \frac{1}{\mathrm{e}^{\beta \omega}-1},
$$

que é o resultado final desejado. A soma sobre as frequiências foi feita, o que restou foi um termo independente da temperatura e outro dependente.

\section{B.3 Soma de freqüências para férmions}

Para o caso de férmions a relação análoga de (B.5) é:

$$
\begin{aligned}
T \sum_{n=-\infty}^{\infty} h\left(p_{0}=i \omega_{n}\right)= & \frac{1}{2 \pi i} \int_{-i \infty}^{+i \infty} \mathrm{d} p_{0} \frac{1}{2}\left[h\left(p_{0}\right)+h\left(-p_{0}\right)\right] \\
& -\frac{1}{2 \pi i} \int_{-i \infty+\epsilon}^{+i \infty+\epsilon} \mathrm{d} p_{0}\left[h\left(p_{0}\right)+h\left(-p_{0}\right)\right] \frac{1}{\mathrm{e}^{\beta p_{0}}+1}
\end{aligned}
$$

onde $h\left(p_{0}\right)$ é uma função que não possui singularidades ao longo do eixo imaginário de $p_{0}$ e $\omega_{n}=\frac{(2 n+1) \pi}{\beta}$. A demonstração pode ser feita seguindo os mesmos passos do caso dos bósons. O análogo de (B.6) será:

$$
T \sum_{n=-\infty}^{\infty} h\left(p_{0}=i \omega_{n}\right)=\frac{T}{2 \pi i} \oint_{C} \mathrm{~d} p_{0} h\left(p_{0}\right) \frac{1}{2} \beta \tanh \left(\frac{1}{2} \beta p_{0}\right),
$$

pois agora é a função tanh que possui pólos em $p_{0}=\frac{(2 n+1) \pi i}{\beta}$.

Para o caso específico em que $h$ é da forma

$$
h\left(p_{0}\right)=\frac{-1}{p_{0}^{2}-\omega^{2}}
$$

obtemos

$$
T \sum_{n=-\infty}^{\infty} \frac{-1}{p_{0}^{2}-\omega^{2}}=\frac{1}{2 \pi i} \int_{-\infty}^{+\infty} \frac{1}{p_{4}^{2}+\omega^{2}} \mathrm{~d} p_{4}-\frac{1}{\omega} \frac{1}{\mathrm{e}^{\beta \omega}+1} .
$$




\section{Apêndice C}

\section{C.1 Integrais úteis}

As integrais seguintes podem ser obtidas na ref. [65]:

$$
\begin{aligned}
& \int_{0}^{\infty} \mathrm{d} x \frac{x}{e^{\frac{x}{T}}-1}=\frac{\pi^{2} T^{2}}{6} \\
& \int_{0}^{\infty} \mathrm{d} x \frac{\sin x y}{e^{\frac{x}{T}}-1}=\frac{\pi T}{2}\left(\operatorname{coth} \pi y T-\frac{1}{\pi y T}\right) \\
& \int_{0}^{\infty} \mathrm{d} x \frac{x \cos x y}{e^{\frac{x}{T}}-1}=\frac{1}{2 y^{2}}-\frac{\pi^{2} T^{2}}{2} \operatorname{cosech}^{2} \pi y T
\end{aligned}
$$

\section{C.2 Somas sobre freqüiências}

A primeira destas relações foi deduzida no Apêndice B.2, equações (B.14)-(B.21). As demais seguem por derivação dos dois lados com relação a $k$.

$$
\begin{aligned}
T \sum_{n} \frac{1}{(2 \pi n T)^{2}+k^{2}}=\frac{n_{B}(k)}{k}+(\text { termo } T=0) \\
T \sum_{n} \frac{1}{\left((2 \pi n T)^{2}+k^{2}\right)^{2}}=-\frac{1}{2 k}\left(\frac{n_{B}(k)}{k}\right)^{\prime}+(\text { termo } T=0) \\
T \sum_{n} \frac{1}{\left((2 \pi n T)^{2}+k^{2}\right)^{3}}=\frac{1}{4 k}\left[\frac{1}{2 k}\left(\frac{n_{B}(k)}{k}\right)^{\prime}\right]^{\prime}+(\text { termo } T=0) \\
T \sum_{n} \frac{1}{\left((2 \pi n T)^{2}+k^{2}\right)^{4}}=-\frac{1}{6 k}\left[\frac{1}{4 k}\left[\frac{1}{2 k}\left(\frac{n_{B}(k)}{k}\right)^{\prime}\right]^{\prime}\right]^{\prime}+(\text { termo } T=0)
\end{aligned}
$$

onde $k=|\vec{k}|$ e o símbolo (') significa derivada com relação a $k$. 


\section{C.3 Expansões em série e identidades trigonométricas}

$$
\begin{gathered}
\cos x=1-\frac{x^{2}}{2 !}+\frac{x^{4}}{4 !}-\frac{x^{6}}{6 !}+\cdots \\
\sin x=x-\frac{x^{3}}{3 !}+\frac{x^{5}}{5 !}-\frac{x^{7}}{7 !}+\cdots \\
\mathrm{e}^{x}=1+x+\frac{x^{2}}{2 !}+\frac{x^{3}}{3 !}+\cdots \\
\operatorname{coth}(x) \simeq \frac{1}{x}+\frac{1}{3} x-\frac{1}{45} x^{3}+\frac{2}{945} x^{5}+\cdots \\
\operatorname{cossech}^{2}(x) \simeq \frac{1}{x^{2}}-\frac{1}{3}+\frac{1}{15} x^{2}-\frac{2}{189} x^{4}+\cdots \\
\sin ^{2} A=\frac{1}{2}(1-\cos 2 A) \\
\end{gathered}
$$

\section{C.4 Integral de auto-energia do fóton}

O cálculo desta integral pode ser encontrado na referência [7], pag. 460.

$$
\begin{aligned}
f(x) & \equiv 6 \int_{0}^{1} z(1-z) \ln (1+x z(1-z)) \mathrm{d} z \\
& =\left[-\frac{5}{3}+\frac{1}{\rho}+\left(1-\frac{1}{2 \rho}\right) \sqrt{1+\frac{1}{\rho}} \ln \frac{\sqrt{1+(1 / \rho)}+1}{\sqrt{1+(1 / \rho)}-1}\right]
\end{aligned}
$$

onde $\rho=x / 4$. Alguns limites importantes são:

$$
f(x) \approx \begin{cases}x / 5 & , \\ \ln x & , \quad x>>1\end{cases}
$$




\section{Apêndice D}

\section{D.1 Método da soma sobre freqüências para a auto-energia do fóton}

Vamos calcular separadamente cada uma das contribuições para o tensor de auto-energia do fóton usando diretamente o método da soma sobre as frequiências de Matsubara. Começamos com o tensor $\Pi_{a}^{\mu \nu}$ dado em (2.66), representado pela Figura 2.2:

$$
\Pi_{a}^{\mu \nu}(p)=4 e^{2} \int \frac{\mathrm{d}^{4} k}{(2 \pi)^{4}} \sin ^{2}\left(\frac{k \times p}{2}\right) \frac{\left(k^{\mu}+p^{\mu}\right) k^{\nu}}{k^{2}(k+p)^{2}} .
$$

Em primeiro lugar, temos de ir para o espaço euclidiano, fazendo a substituição $k^{\mu}=\left(k^{0}, \vec{k}\right) \rightarrow\left(i \omega_{n}, \vec{k}\right)$ de maneira que $k^{2}=\left(k_{0}^{2}-\vec{k}^{2}\right) \rightarrow-\left(\omega_{n}^{2}+\vec{k}^{2}\right)$, onde $\omega_{n}=2 n \pi / \beta$, e da mesma forma $p^{\mu}=\left(p^{0}, \vec{p}\right) \rightarrow$ $\left(i p_{0}, \vec{p}\right)$. A parte trigonométrica do tensor não muda, pois ela só depende das componentes espaciais. Assim temos

$$
\Pi_{a}^{\mu \nu}(p)=4 e^{2} \int \frac{\mathrm{d}^{4} k}{(2 \pi)^{4}} \sin ^{2}\left(\frac{k \times p}{2}\right) \frac{(k+p)^{\mu} k^{\nu}}{\left(-\omega_{n}^{2}-\vec{k}^{2}\right)\left[-\left(\omega_{n}+p_{0}\right)^{2}-(\vec{k}+\vec{p})^{2}\right]},
$$

e fazemos a substituição

$$
\int \frac{\mathrm{d}^{4} k}{(2 \pi)^{4}} \rightarrow \frac{1}{\beta} \sum_{n=-\infty}^{\infty} \int \frac{\mathrm{d}^{3} k}{(2 \pi)^{3}}
$$

obtendo

$$
\Pi_{a}^{\mu \nu}(p)=4 e^{2} \frac{1}{\beta} \int \frac{\mathrm{d}^{3} k}{(2 \pi)^{3}} \sin ^{2}\left(\frac{k \times p}{2}\right) \sum_{n} \frac{(k+p)^{\mu} k^{\nu}}{\left(\omega_{n}^{2}+\vec{k}^{2}\right)\left[\left(\omega_{n}+p_{0}\right)^{2}+(\vec{k}+\vec{p})^{2}\right]}
$$

de maneira que podemos fazer os cálculos sobre a soma das frequiências $\omega_{n}$. Note que o numerador do integrando ainda contém uma dependência nas frequiências, por exemplo se quisermos calcular a componente $\Pi^{00}$ do tensor. Mas esta dependência no numerador pode ser facilmente simplificada, como veremos 
adiante. Vamos começar calculando a parte espacial do tensor,

$$
\begin{aligned}
& \Pi_{a}^{i j}(p)=4 e^{2} \frac{1}{\beta} \int \frac{\mathrm{d}^{3} k}{(2 \pi)^{3}} \sin ^{2}\left(\frac{k \times p}{2}\right) \sum_{n} \frac{k^{i} k^{j}+p^{i} k^{j}}{\left(\omega_{n}^{2}+\vec{k}^{2}\right)\left[\left(\omega_{n}+p_{0}\right)^{2}+(\vec{k}+\vec{p})^{2}\right]} \\
& =4 e^{2} \int \frac{\mathrm{d}^{3} k}{(2 \pi)^{3}} \sin ^{2}\left(\frac{k \times p}{2}\right)\left(k^{i} k^{j}+p^{i} k^{j}\right) \frac{1}{\beta} \sum_{n} \frac{1}{\left((2 \pi n / \beta)^{2}+\vec{k}^{2}\right)\left[\left(2 \pi n / \beta+p_{0}\right)^{2}+(\vec{k}+\vec{p})^{2}\right]}
\end{aligned}
$$

Esta somatória será feita no chamado limite estático, que corresponde a fazer $p^{0}=0$ e em seguida $\vec{p} \rightarrow 0$. O caso inverso, $\vec{p}=0, p^{0} \rightarrow 0$ fornece um outro limite ${ }^{1}$, caracterizando a não-analiticidade de cálculos a temperatura finita. Para o nosso caso, o limite estático corresponde a tomar $p^{0}=\vec{p}=0$ no numerador de (D.5) e fazer a soma. Mas isto se reduz à expressão (C.5) do Apêndice C.2, de forma que obtemos

$$
\Pi_{a}^{i j}\left(p^{0}=0, \vec{p} \rightarrow 0\right)=2 e^{2} \int \frac{\mathrm{d}^{3} k}{(2 \pi)^{3}} \sin ^{2}\left(\frac{k \times p}{2}\right) \frac{k^{i} k^{j}}{|\vec{k}|^{3}}\left[\frac{1}{2}+n_{B}(|\vec{k}|)-|\vec{k}| n_{B}^{\prime}(|\vec{k}|)\right] .
$$

O fator $1 / 2$ representa a parte independente da temperatura, que dará contribuição para a parte de vácuo. Portanto, desconsiderando a parte independente da temperatura obtemos, para as componentes espaciais,

$$
\Pi_{a \mathrm{st}}^{i j}(p)=2 e^{2} \int \frac{\mathrm{d}^{3} k}{(2 \pi)^{3}} \sin ^{2}\left(\frac{k \times p}{2}\right) \frac{k^{i} k^{j}}{|\vec{k}|^{3}}\left[n_{B}(|\vec{k}|)-|\vec{k}| n_{B}^{\prime}(|\vec{k}|)\right]
$$

lembrando que a única dependência com o momento externo está agora dentro do fator trigonométrico, pois queremos ter alguma informação sobre a não-comutatividade no resultado final.

Vejamos agora a parte temporal do tensor dado em (D.4). Ela pode ser escrita como

$$
\begin{aligned}
\Pi_{a}^{00}(p) & =4 e^{2} \frac{1}{\beta} \int \frac{\mathrm{d}^{3} k}{(2 \pi)^{3}} \sin ^{2}\left(\frac{k \times p}{2}\right) \sum_{n} \frac{k^{0} k^{0}+p^{0} k^{0}}{\left(\omega_{n}^{2}+\vec{k}^{2}\right)\left[\left(\omega_{n}+p_{0}\right)^{2}+(\vec{k}+\vec{p})^{2}\right]} \\
& =4 e^{2} \frac{1}{\beta} \int \frac{\mathrm{d}^{3} k}{(2 \pi)^{3}} \sin ^{2}\left(\frac{k \times p}{2}\right) \sum_{n} \frac{-\omega_{n}^{2}-p_{0} \omega_{n}}{\left(\omega_{n}^{2}+\vec{k}^{2}\right)\left[\left(\omega_{n}+p_{0}\right)^{2}+(\vec{k}+\vec{p})^{2}\right]}
\end{aligned}
$$

mas é fácil ver que a soma sobre o segundo termo, linear em $\omega_{n}$ no numerador, é nula, pois o denominador é uma função par em $\omega_{n}$ enquanto o numerador é uma função ímpar, então a soma que vai de $-\infty$ até $+\infty$ se anula. Para o primeiro termo é mais conveniente escrevermos

$$
\begin{gathered}
\Pi_{a}^{00}(p)=4 e^{2} \frac{1}{\beta} \int \frac{\mathrm{d}^{3} k}{(2 \pi)^{3}} \sin ^{2}\left(\frac{k \times p}{2}\right) \sum_{n} \frac{-\omega_{n}^{2}-\vec{k}^{2}+\vec{k}^{2}}{\left(\omega_{n}^{2}+\vec{k}^{2}\right)\left[\left(\omega_{n}+p_{0}\right)^{2}+(\vec{k}+\vec{p})^{2}\right]} \\
=4 e^{2} \frac{1}{\beta} \int \frac{\mathrm{d}^{3} k}{(2 \pi)^{3}} \sin ^{2}\left(\frac{k \times p}{2}\right) \sum_{n}\left[\frac{-1}{\left[\left(\omega_{n}+p_{0}\right)^{2}+(\vec{k}+\vec{p})^{2}\right]}\right. \\
\left.+\frac{\vec{k}^{2}}{\left(\omega_{n}^{2}+\vec{k}^{2}\right)\left[\left(\omega_{n}+p_{0}\right)^{2}+(\vec{k}+\vec{p})^{2}\right]}\right] .
\end{gathered}
$$

No limite estático as somas se reduzem àquelas do Apêndice C.2. Desconsiderando a parte independente

${ }^{1}$ Conforme discutido na seção 3.2. 
da temperatura temos,

$$
\Pi_{a \mathrm{st}}^{00}(p)=2 e^{2} \int \frac{\mathrm{d}^{3} k}{(2 \pi)^{3}} \sin ^{2}\left(\frac{k \times p}{2}\right) \frac{|\vec{k}|^{2}}{|\vec{k}|^{3}}\left[n_{B}(|\vec{k}|)-|\vec{k}| n_{B}^{\prime}(|\vec{k}|)-2 n_{B}(|\vec{k}|)\right]
$$

Ficou faltando calcularmos as componentes do tipo $\Pi^{0 j}$, mas é fácil ver que todas as componentes se anulam, pois o denominador é uma função par em $\omega_{n}$ enquanto o numerador é uma função ímpar. Além disto, no limite de altas temperaturas, que corresponde a fazer $p<<k$, toda dependência em $p$ no numerador pode ser desconsiderada.

Dessa maneira, olhando atentamente para (D.7) e (D.10), percebemos que o tensor do diagrama (a) no limite estático pode ser escrito compactamente como

$$
\Pi_{a \mathrm{st}}^{\mu \nu}(p)=\left.2 e^{2} \int \frac{\mathrm{d}^{3} k}{(2 \pi)^{3}} \sin ^{2}\left(\frac{k \times p}{2}\right)\left[\frac{n_{B}(|\vec{k}|)}{|\vec{k}|^{3}} k^{\mu} k^{\nu}-\frac{n_{B}^{\prime}(|\vec{k}|)}{|\vec{k}|^{2}} k^{\mu} k^{\nu}-2 \frac{n_{B}(|\vec{k}|)}{|\vec{k}|} \eta^{\mu 0} \eta_{0}^{\nu}\right]\right|_{k^{0}=|\vec{k}|}
$$

onde $k^{0}=|\vec{k}|$, ou seja, está na camada de massa. Note que o último termo só contribui para a componente $\Pi^{00}$ do tensor.

Para o gráfico da Figura 2.4(b), o tensor a temperatura nula é dado pela equação (2.67). Fazendo as mesmas substituições e usando as mesmas técnicas do exemplo anterior, obtemos, a temperatura finita e no limite estático,

$$
\Pi_{b \mathrm{st}}^{\mu \nu}(p)=-12 e^{2} \int \frac{\mathrm{d}^{3} k}{(2 \pi)^{3}} \sin ^{2}\left(\frac{k \times p}{2}\right) \frac{n_{B}(|\vec{k}|)}{|\vec{k}|} \eta^{\mu \nu}
$$

Para calcular a contribuição do diagrama da Figura 2.1(c), dado pela equação (2.68), vamos usar desde o início que estamos interessados no limite $p<<k$, de maneira que toda dependência em $p$ no numerador pode ser desconsiderada. Desta maneira o ponto de partida é a expressão

$$
\Pi_{c}^{\mu \nu}(p)=-2 e^{2} \int \frac{\mathrm{d}^{4} k}{(2 \pi)^{4}} \sin ^{2}\left(\frac{k \times p}{2}\right)\left[\frac{2 \eta^{\mu \nu}}{(k+p)^{2}}+\frac{10 k^{\mu} k^{\nu}}{k^{2}(k+p)^{2}}\right]
$$

Agora fazemos as mesmas substituições que nos casos anteriores e vamos obter, no limite estático,

$$
\begin{gathered}
\Pi_{c \mathrm{st}}^{\mu \nu}(p)=2 e^{2} \int \frac{\mathrm{d}^{3} k}{(2 \pi)^{3}} \\
\sin ^{2}\left(\frac{k \times p}{2}\right)\left[2 \frac{n_{B}(|\vec{k}|)}{|\vec{k}|} \eta^{\mu \nu}-5 \frac{n_{B}(|\vec{k}|)}{|\vec{k}|^{3}} k^{\mu} k^{\nu}+5 \frac{n_{B}^{\prime}(|\vec{k}|)}{|\vec{k}|^{2}} k^{\mu} k^{\nu}\right. \\
\left.+10 \frac{n_{B}(|\vec{k}|)}{|\vec{k}|} \eta^{\mu 0} \eta_{0}^{\nu}\right]\left.\right|_{k^{0}=|\vec{k}|} .
\end{gathered}
$$

Portanto, a contribuição total para o tensor de auto-energia do fóton no limite estático é dada finalmente 
por

$$
\begin{aligned}
& \Pi_{\mathrm{st}}^{\mu \nu}(p)=\Pi_{a \mathrm{st}}^{\mu \nu}(p)+\Pi_{b \mathrm{st}}^{\mu \nu}(p)+\Pi_{c \mathrm{st}}^{\mu \nu}(p) \\
& =-8 e^{2} \int \frac{\mathrm{d}^{3} k}{(2 \pi)^{3}} \sin ^{2}\left(\frac{k \times p}{2}\right) \frac{1}{|\vec{k}|}\left[n_{B}(|\vec{k}|) \eta^{\mu \nu}+\frac{n_{B}(|\vec{k}|)}{|\vec{k}|^{2}} k^{\mu} k^{\nu}-\frac{n_{B}^{\prime}(|\vec{k}|)}{|\vec{k}|} k^{\mu} k^{\nu}\right. \\
& \left.-2 n_{B}(|\vec{k}|) \eta^{\mu 0} \eta_{0}^{\nu}\right]\left.\right|_{k^{0}=|\vec{k}|} .
\end{aligned}
$$

\section{D.2 Integrais da auto-energia do fóton}

Vamos calcular os fatores de forma $\Pi_{\mathrm{L}}^{\mathrm{st}}$, $\tilde{\Pi}_{\mathrm{T}}^{\mathrm{st}} \mathrm{e} \Pi_{\mathrm{T}}^{\mathrm{st}}$ da seção 3.2. O vetor momento $\vec{p}$ é perpendicular a $\overrightarrow{\tilde{p}}$, e o momento a ser integrado é $\vec{k}$. Portanto, um sistema de coordenadas conveniente para fazermos os cálculos é representado na Figura D.1.

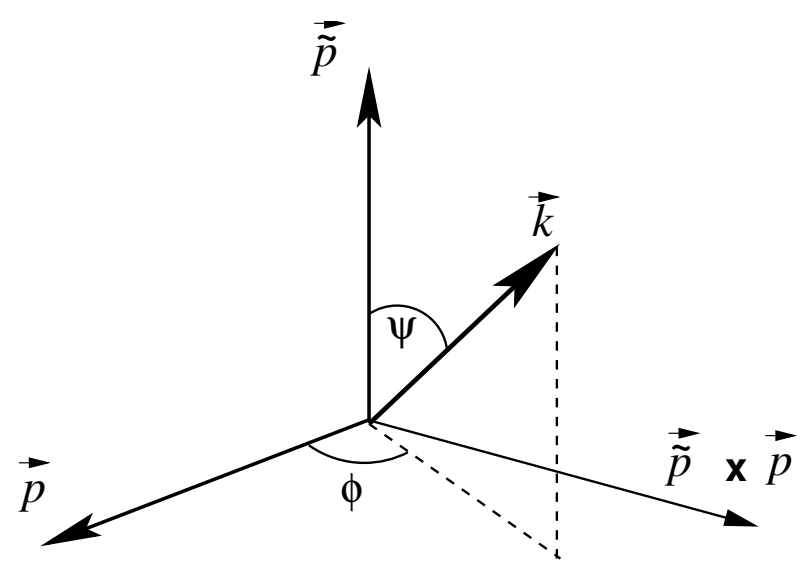

Figura D.1: Sistema de coordenadas usado para fazer a integração no momento interno $k$. 


$$
\begin{aligned}
& \Pi_{\mathrm{L}}^{\mathrm{st}}=u_{\mu} u_{\nu} \Pi_{S t}^{\mu \nu} \\
& =8 e^{2} \int \frac{\mathrm{d}^{3} k}{(2 \pi)^{3}} \sin ^{2}\left(\frac{k \times p}{2}\right) n_{B}^{\prime}(|\vec{k}|) \\
& =\frac{4 e^{2}}{(2 \pi)^{3}} \int \mathrm{d}^{3} k(1-\cos \vec{k} \cdot \overrightarrow{\tilde{p}}) \frac{\mathrm{d}}{\mathrm{d}|\vec{k}|} n_{B}(|\vec{k}|) \\
& =\frac{4 e^{2}}{(2 \pi)^{3}} \int \mathrm{d}^{3} k(1-\cos \vec{k} \cdot \overrightarrow{\tilde{p}}) \beta \frac{\mathrm{d}}{\mathrm{d} \beta} \frac{n_{B}(|\vec{k}|)}{|\vec{k}|} \\
& =\frac{4 e^{2}}{(2 \pi)^{3}} \beta \frac{\mathrm{d}}{\mathrm{d} \beta} \int_{0}^{\infty} \int_{0}^{\pi} \int_{0}^{2 \pi} \mathrm{d} k \mathrm{~d} \psi \mathrm{d} \phi k^{2} \sin \psi \frac{n_{B}(k)}{k}[1-\cos (|\overrightarrow{\tilde{p}}| k \cos \psi)] \\
& =\frac{8 e^{2}}{(2 \pi)^{2}} \beta \frac{\mathrm{d}}{\mathrm{d} \beta} \int_{0}^{\infty} \frac{\mathrm{d} k k}{e^{\beta k}-1}\left(1-\frac{\sin k|\overrightarrow{\tilde{p}}|}{k|\overrightarrow{\tilde{p}}|}\right) \\
& =\frac{8 e^{2}}{(2 \pi)^{2}} \beta \frac{\mathrm{d}}{\mathrm{d} \beta}\left[\frac{\pi^{2}}{6 \beta^{2}}-\frac{\pi}{2|\overrightarrow{\tilde{p}}| \beta} \operatorname{coth}\left(\frac{\pi|\overrightarrow{\tilde{p}}|}{\beta}\right)+\frac{1}{2|\overrightarrow{\tilde{p}}|^{2}}\right] \\
& =-\frac{2}{3} e^{2} T^{2}+\frac{e^{2} T}{\pi|\overrightarrow{\tilde{p}}|} \operatorname{coth}(\pi|\overrightarrow{\tilde{p}}| T)-e^{2} T^{2} \operatorname{cossech}^{2}(\pi|\overrightarrow{\tilde{p}}| T) \\
& \tilde{\Pi}_{\mathrm{T}}^{\mathrm{st}}=\frac{\tilde{p}_{\mu} \tilde{p}_{\nu}}{\tilde{p}^{2}} \Pi_{S t}^{\mu \nu} \\
& =-8 e^{2} \int \frac{\mathrm{d}^{3} k}{(2 \pi)^{3}} \sin ^{2}\left(\frac{k \times p}{2}\right)\left[\frac{n_{B}(|\vec{k}|)}{|\vec{k}|}+\frac{(\tilde{p} \cdot k)^{2}}{\tilde{p}^{2}} \frac{n_{B}(|\vec{k}|)}{|\vec{k}|^{3}}-\frac{(\tilde{p} \cdot k)^{2}}{\tilde{p}^{2}} \frac{n_{B}^{\prime}(|\vec{k}|)}{|\vec{k}|^{2}}\right] \\
& =-\frac{2 e^{2}}{\pi^{2}|\overrightarrow{\tilde{p}}|^{2}}+\frac{e^{2} T}{\pi|\overrightarrow{\tilde{p}}|} \operatorname{coth}(\pi|\overrightarrow{\tilde{p}}| T)+e^{2} T^{2} \operatorname{cossech}^{2}(\pi|\overrightarrow{\tilde{p}}| T) \\
& \Pi_{\mathrm{T}}^{\mathrm{st}}=\left(\eta_{\mu \nu}-\frac{p_{\mu} p_{\nu}}{p^{2}}\right) \Pi_{\mathrm{st}}^{\mu \nu}-\Pi_{\mathrm{L}}^{\mathrm{st}}-\tilde{\Pi}_{\mathrm{T}}^{\mathrm{st}} \\
& =-8 e^{2} \int \frac{\mathrm{d}^{3} k}{(2 \pi)^{3}} \sin ^{2}\left(\frac{k \times p}{2}\right)\left[n_{B}^{\prime}(|\vec{k}|)+\left(\frac{(\tilde{p} \cdot k)^{2}}{\tilde{p}^{2}}+\frac{(p \cdot k)^{2}}{p^{2}}\right)\left(\frac{n_{B}^{\prime}(|\vec{k}|)}{|\vec{k}|^{2}}-\frac{n_{B}(|\vec{k}|)}{|\vec{k}|^{3}}\right)\right] \\
& =0 \text {. }
\end{aligned}
$$

onde $k=|\vec{k}|, \beta=1 / T$ e usamos os resultados das integrais dada no Apêndice C.1. 


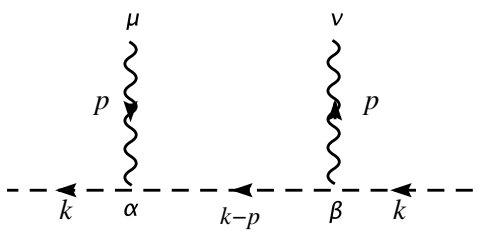

$(a i)$

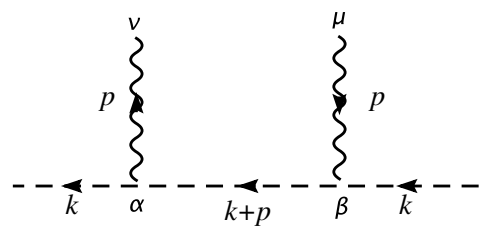

(aii)

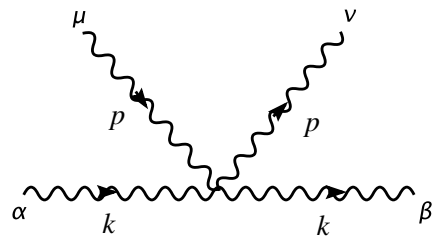

(b)

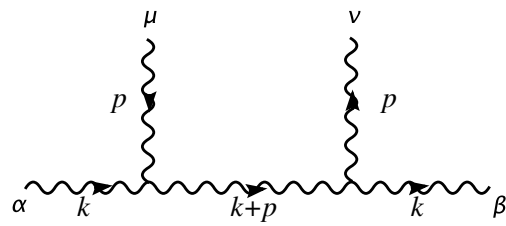

$(c i)$

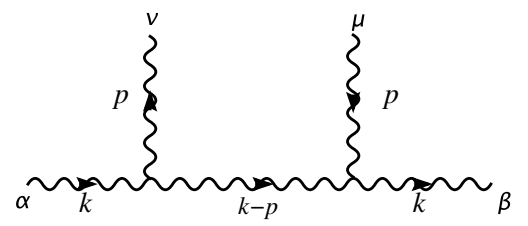

(cii)

Figura D.2: Contribuição para o espalhamento frontal

\section{D.3 Método da amplitude de espalhamento frontal}

Neste método, a função de dois pontos para a auto-energia do fóton pode ser escrita como

$$
\Pi^{\mu \nu}(p)=-\left.\frac{1}{2 i(2 \pi)^{3}} \int \frac{\mathrm{d}^{3} \vec{k}}{|\vec{k}|} n_{B}(|\vec{k}|) P^{\alpha \beta}(k) A_{\alpha \beta}^{\mu \nu}(k,-k, p)\right|_{k_{0}=|\vec{k}|}
$$

onde $P^{\alpha \beta}(k)$ é um operador que leva em conta a dependência de gauge e é dado por

$$
P^{\alpha \beta}(k) \equiv \eta^{\alpha \beta}-\frac{1-\xi}{2} \frac{\mathrm{d}}{\mathrm{d} k_{0}} \frac{k^{\alpha} k^{\beta}}{k_{0}}
$$

e $A_{\alpha \beta}^{\mu \nu}(k,-k, p)$ é a amplitude de espalhamento frontal, que é obtida dos correspondentes gráficos que representam estes espalhamentos calculados na camada de massa $\left(k_{0}=|\vec{k}|\right)$. Para os diagramas da Figura 2.1, os correspondentes gráficos que representam o espalhamento frontal estão indicados na Figura D.2. Os diagramas (ai) e (aii) representam os gráficos de espalhamento frontal que contribuem para o loop de ghost da Figura 2.1(a). Além deles devemos levar em conta também os mesmos diagramas fazendo $k \rightarrow-k$, por isso o fator $-k$ presente no argumento da amplitude $A_{\alpha \beta}^{\mu \nu}$. O diagrama (b) representa o espalhameto frontal da Figura 2.1(b) e os diagramas (ci) e (cii) são os correspondentes para o diagrama da Figura 2.1 $(c)$. Devemos ainda considerar os fatores combinatoriais presentes nos gráficos da Figura 2.1. O fator (-1) do loop de ghost não se trata de um fator combinatorial, e já está levado em conta no sentido da seta nos diagramas (ai) e (aii) da Figura D.2. Vamos agora calcular explicitamente estas amplitudes.

Começando pelo diagrama mais simples, a contribuição do diagrama (b) para a amplitude é dada por, usando as regras de Feynman

$$
P^{\alpha \beta} A_{\alpha \beta(b)}^{\mu \nu}(k, p)=-4 e^{2} \sin ^{2}\left(\frac{k \times p}{2}\right)\left(6 \eta^{\mu \nu}\right)
$$


onde estamos trabalhando no gauge de Feynman, $\xi=1$ e por simplicidade já foi feita a contração com o operador $P^{\alpha \beta}$.

Os diagramas $(c i)$ e (cii) darão contribuições do tipo

$$
\begin{aligned}
& P^{\alpha \beta} A_{\alpha \beta(c i)}^{\mu \nu}(k, p) \\
& \quad=-4 e^{2} \sin ^{2}\left(\frac{k \times p}{2}\right) \frac{\left(-5 p^{2}-2 k \cdot p-2 k^{2}\right) \eta^{\mu \nu}+2 p^{\mu} p^{\nu}-5\left(k^{\mu} p^{\nu}+p^{\mu} k^{\nu}\right)-10 k^{\mu} k^{\nu}}{(k+p)^{2}} \\
& P^{\alpha \beta} A_{\alpha \beta(c i i)}^{\mu \nu}(k, p) \\
& \quad=-4 e^{2} \sin ^{2}\left(\frac{k \times p}{2}\right) \frac{\left(-5 p^{2}+2 k \cdot p-2 k^{2}\right) \eta^{\mu \nu}+2 p^{\mu} p^{\nu}+5\left(k^{\mu} p^{\nu}+p^{\mu} k^{\nu}\right)-10 k^{\mu} k^{\nu}}{(k-p)^{2}}
\end{aligned}
$$

Para os diagramas (ai) e (aii) que descrevem a propagação de um ghost, é mais conveniente redefinir a amplitude $A_{\alpha \beta}^{\mu \nu}(k, p)$ como

$$
A_{\alpha \beta}^{\mu \nu}(k, p) \equiv \frac{\eta_{\alpha \beta}}{3+\xi} A_{g h o s t}^{\mu \nu}(k, p)
$$

onde $A_{\text {ghost }}^{\mu \nu}(k, p)$ é amplitude de espalhamento frontal de um ghost pelo campo do fóton. Desta maneira, os diagramas (ai) e (aii) fornecem, no gauge de Feynman,

$$
\begin{gathered}
P^{\alpha \beta} A_{\alpha \beta(a i)}^{\mu \nu}(k, p)=-4 e^{2} \sin ^{2}\left(\frac{k \times p}{2}\right) \frac{k^{\mu}(k-p)^{\nu}}{(k-p)^{2}} \\
P^{\alpha \beta} A_{\alpha \beta(a i i)}^{\mu \nu}(k, p)=-4 e^{2} \sin ^{2}\left(\frac{k \times p}{2}\right) \frac{k^{\nu}(k+p)^{\mu}}{(k+p)^{2}}
\end{gathered}
$$

Lembrando que a contribuição total ainda tem de levar em conta os diagramas obtidos da Figura D.2 fazendo $k \rightarrow-k$, a amplitude total de cada diagrama é obtida por meio de

$$
P^{\alpha \beta} A_{\alpha \beta}^{\mu \nu}(k,-k, p)=P^{\alpha \beta} A_{\alpha \beta}^{\mu \nu}(k, p)+P^{\alpha \beta} A_{\alpha \beta}^{\mu \nu}(-k, p) .
$$

Portanto, levando isto em consideração e também os fatores combinatorias de cada gráfico, a contribuição total para a auto-energia do fóton obtido por meio de (D.19) é, no gauge de Feynman e na camada de massa $k^{2}=0$,

$$
\begin{aligned}
\Pi^{\mu \nu}(p)= & \frac{4 e^{2}}{(2 \pi)^{3}} \int \frac{\mathrm{d}^{3} \vec{k}}{2|\vec{k}|} n_{B}(|\vec{k}|) \sin ^{2}\left(\frac{k \times p}{2}\right)\left[-6 \eta^{\mu \nu}\right. \\
& +\frac{\left(5 p^{2}+2 k \cdot p\right) \eta^{\mu \nu}-2 p^{\mu} p^{\nu}+4\left(k^{\mu} p^{\nu}+p^{\mu} k^{\nu}\right)+8 k^{\mu} k^{\nu}}{p^{2}+2 k \cdot p} \\
& \left.+\frac{\left(5 p^{2}-2 k \cdot p\right) \eta^{\mu \nu}-2 p^{\mu} p^{\nu}-4\left(k^{\mu} p^{\nu}+p^{\mu} k^{\nu}\right)+8 k^{\mu} k^{\nu}}{p^{2}-2 k \cdot p}\right]\left.\right|_{k_{0}=|\vec{k}|}
\end{aligned}
$$


Para continuarmos nossos cálculos vamos agora tomar o limite de altas temperaturas ou limite HTL onde $p \ll k \sim T$, de maneira que vale a seguinte expansão para o denominador

$$
\frac{1}{p^{2} \pm 2 k \cdot p}= \pm \frac{1}{2 k \cdot p}-\frac{p^{2}}{(2 k \cdot p)^{2}}+\cdots
$$

Usando isto na (D.26), o tensor de auto-energia do fóton a altas temperaturas pode ser escrito como

$$
\Pi^{\mu \nu}(p)=-\left.\frac{4 e^{2}}{(2 \pi)^{3}} \int \frac{\mathrm{d}^{3} \vec{k}}{|\vec{k}|} n_{B}(|\vec{k}|)(1-\cos k \times p)\left[\eta^{\mu \nu}-\frac{p^{\mu} k^{\nu}+p^{\nu} k^{\mu}}{p \cdot k}+\frac{p^{2} k^{\mu} k^{\nu}}{(p \cdot k)^{2}}\right]\right|_{k^{0}=|\vec{k}|}
$$




\section{Apêndice E}

\section{E.1 Amplitude de 3-pontos}

Nosso objetivo é demonstrar as relações (3.33) e (3.34) satisfeita pela amplitude de 3-pontos. Partimos da expressão (3.32):

$$
\begin{aligned}
\Gamma_{\mu \nu \lambda}^{\mathrm{st}}= & i e^{3} T \int \frac{\mathrm{d}^{3} k}{(2 \pi)^{3}} \sin \left(\frac{\tilde{p}_{1} \cdot k}{2}\right) \sin \left(\frac{\tilde{p}_{2} \cdot k}{2}\right) \sin \left(\frac{\tilde{p}_{3} \cdot k}{2}\right) \sum_{n}\left[\frac{128 k_{\mu} k_{\nu} k_{\lambda}}{\left[(2 \pi n T)^{2}+k^{2}\right]^{3}}\right. \\
& \left.-\frac{32}{\left[(2 \pi n T)^{2}+k^{2}\right]^{2}}\left(\delta_{\mu \nu} k_{\lambda}+\delta_{\lambda \mu} k_{\nu}+\delta_{\nu \lambda} k_{\mu}\right)\right] .
\end{aligned}
$$

Fazendo uso da identidade trigonométrica

$$
\sin \left(\frac{\tilde{p}_{1} \cdot k}{2}\right) \sin \left(\frac{\tilde{p}_{2} \cdot k}{2}\right) \sin \left(\frac{\tilde{p}_{3} \cdot k}{2}\right)=-\frac{1}{4}\left(\sin \tilde{p}_{1} \cdot k+\sin \tilde{p}_{2} \cdot k+\sin \tilde{p}_{3} \cdot k\right)
$$

podemos reescrever (E.1) como

$$
\begin{gathered}
\Gamma_{\mu \nu \lambda}^{\mathrm{st}}=-8 i e^{3} T \int \frac{\mathrm{d}^{3} k}{(2 \pi)^{3}}\left(\sin \tilde{p}_{1} \cdot k+\sin \tilde{p}_{2} \cdot k+\sin \tilde{p}_{3} \cdot k\right) \sum_{n}\left[\frac{4 k_{\mu} k_{\nu} k_{\lambda}}{\left[(2 \pi n T)^{2}+k^{2}\right]^{3}}\right. \\
\left.-\frac{1}{\left[(2 \pi n T)^{2}+k^{2}\right]^{2}}\left(\delta_{\mu \nu} k_{\lambda}+\delta_{\lambda \mu} k_{\nu}+\delta_{\nu \lambda} k_{\mu}\right)\right] .
\end{gathered}
$$

Podemos ver desta expressão que quando houver um número ímpar de índices temporais a amplitude se anula, pois a soma sobre as freqüências será antissimétrica, sendo uma função par no denominador e ímpar no numerador, de forma que se anula para $n$ variando no intervalo $-\infty<n<+\infty$. As somas explícitas sobre as frequiências podem ser feitas usando-se os resultados do Apêndice D.2. No entanto, note que a expressão (E.3) pode ser escrita como derivadas de uma função logarítmica, na forma

$$
\Gamma_{\mu \nu \lambda}^{\mathrm{st}}=-2 i e^{3} T \int \frac{\mathrm{d}^{3} k}{(2 \pi)^{3}}\left(\sin \tilde{p}_{1} \cdot k+\sin \tilde{p}_{2} \cdot k+\sin \tilde{p}_{3} \cdot k\right) \frac{\partial^{3}}{\partial k_{\mu} \partial k_{\nu} \partial k_{\lambda}} \sum_{n} \ln \left[(2 \pi n T)^{2}+k^{2}\right] .
$$


Agora fazemos duas integrações a direita, agindo na função logarítmica, e para a última derivada fazemos uma integração por partes, de forma que obtemos

$$
\begin{aligned}
\Gamma_{\mu \nu \lambda}^{\mathrm{st}}=2 i e^{3} T & \int \frac{\mathrm{d}^{3} k}{(2 \pi)^{3}}\left(\tilde{p}_{1, \mu} \cos \tilde{p}_{1} \cdot k+\tilde{p}_{2, \mu} \cos \tilde{p}_{2} \cdot k+\tilde{p}_{3, \mu} \cos \tilde{p}_{3} \cdot k\right) \\
& \times\left[\sum_{n} \frac{-4 k_{\nu} k_{\lambda}}{\left[(2 \pi n T)^{2}+k^{2}\right]^{2}}+\sum_{n} \frac{2 \eta_{\nu \lambda}}{\left[(2 \pi n T)^{2}+k^{2}\right]}\right] .
\end{aligned}
$$

Usando a relação

$$
\begin{aligned}
-\left(\tilde{p}_{1, \mu} \cos \tilde{p}_{1} \cdot k\right. & \left.+\tilde{p}_{2, \mu} \cos \tilde{p}_{2} \cdot k+\tilde{p}_{3, \mu} \cos \tilde{p}_{3} \cdot k\right) \\
= & \tilde{p}_{1, \mu}\left(1-\cos \tilde{p}_{1} \cdot k\right)+\tilde{p}_{2, \mu}\left(1-\cos \tilde{p}_{2} \cdot k\right)+\tilde{p}_{3, \mu}\left(1-\cos \tilde{p}_{3} \cdot k\right),
\end{aligned}
$$

que pode ser demonstrada usando-se a conservação de momento $p_{1}+p_{2}+p_{3}=0$, vemos que a amplitude de 3-pontos pode ser escrita em termos da auto-energia do fóton no limite estático, na forma

$$
\Gamma_{\mu \nu \lambda}^{\mathrm{st}}\left(p_{1}, p_{2}, p_{3}\right)=i e\left[\tilde{p}_{1, \mu} \Pi_{\nu \lambda}^{\mathrm{st}}\left(p_{1}\right)+\tilde{p}_{2, \mu} \Pi_{\nu \lambda}^{\mathrm{st}}\left(p_{2}\right)+\tilde{p}_{3, \mu} \Pi_{\nu \lambda}^{\mathrm{st}}\left(p_{3}\right)\right]
$$

Desta forma, as componentes não-nulas na ordem dominante que estamos procurando podem ser representadas por:

$$
\begin{aligned}
& \Gamma_{00 i}^{\mathrm{static}}\left(p_{1}, p_{2}, p_{3}\right)=i e\left[\tilde{p}_{1, i} \Pi_{00}^{\mathrm{static}}\left(p_{1}\right)+\tilde{p}_{2, i} \Pi_{00}^{\mathrm{static}}\left(p_{2}\right)+\tilde{p}_{3, i} \Pi_{00}^{\mathrm{static}}\left(p_{3}\right)\right] \\
& \Gamma_{i j k}^{\mathrm{static}}\left(p_{1}, p_{2}, p_{3}\right)=i e\left[\tilde{p}_{1, k} \Pi_{i j}^{\mathrm{static}}\left(p_{1}\right)+\tilde{p}_{2, k} \Pi_{i j}^{\mathrm{static}}\left(p_{2}\right)+\tilde{p}_{3, k} \Pi_{i j}^{\mathrm{static}}\left(p_{3}\right)\right] .
\end{aligned}
$$

\section{E.2 Amplitude de 4-pontos}

Nosso objetivo é demonstrar a relação (3.36) satisfeita pela amplitude de 4-pontos. Partimos da relação

$$
\begin{aligned}
& \Gamma_{\mu \nu \lambda \rho}^{\mathrm{st}}\left(p_{1}, p_{2}, p_{3}, p_{4}\right) \\
& =32 e^{4} \int \frac{\mathrm{d}^{3} k}{(2 \pi)^{3}} \sin \left(\frac{\tilde{p}_{1} \cdot k}{2}\right) \sin \left(\frac{\tilde{p}_{2} \cdot k}{2}\right) \sin \left(\frac{\tilde{p}_{3} \cdot k}{2}\right) \sin \left(\frac{\tilde{p}_{4} \cdot k}{2}\right) T \sum_{n}\left[\frac{24 k_{\mu} k_{\nu} k_{\lambda} k_{\rho}}{\left((2 \pi n T)^{2}+k^{2}\right)^{4}}\right. \\
& -\frac{4}{\left[(2 \pi n T)^{2}+k^{2}\right]^{3}}\left(\delta_{\mu \nu} k_{\lambda} k_{\rho}+\delta_{\rho \mu} k_{\nu} k_{\lambda}+\delta_{\lambda \rho} k_{\mu} k_{\nu}+\delta_{\nu \lambda} k_{\rho} k_{\mu}\right) \\
& \left.+\frac{2}{\left[(2 \pi n T)^{2}+k^{2}\right]^{2}}\left(\delta_{\mu \nu} \delta_{\lambda \rho}+\delta_{\rho \mu} \delta_{\nu \lambda}\right)\right]
\end{aligned}
$$


Esta relação também pode ser escrita em termos de derivadas agindo em uma função logarítmica, da forma

$$
\begin{array}{rl}
\Gamma_{\mu \nu \lambda \rho}^{\mathrm{st}}\left(p_{1}, p_{2}, p_{3}, p_{4}\right)=32 e^{4} & T \int \frac{\mathrm{d}^{3} k}{(2 \pi)^{3}} \sin \left(\frac{\tilde{p}_{1} \cdot k}{2}\right) \sin \left(\frac{\tilde{p}_{2} \cdot k}{2}\right) \sin \left(\frac{\tilde{p}_{3} \cdot k}{2}\right) \sin \left(\frac{\tilde{p}_{4} \cdot k}{2}\right) \\
& \times \frac{\partial^{4}}{\partial k_{\mu} \partial k_{\nu} \partial k_{\lambda} \partial k_{\rho}} \sum_{n} \ln \left[(2 \pi n T)^{2}+k^{2}\right]
\end{array}
$$

O fator trigonométrico do vértice pode ser escrito como

$$
\begin{aligned}
& 8 \sin \left(\frac{\tilde{p}_{1} \cdot k}{2}\right) \sin \left(\frac{\tilde{p}_{2} \cdot k}{2}\right) \sin \left(\frac{\tilde{p}_{3} \cdot k}{2}\right) \sin \left(\frac{\tilde{p}_{4} \cdot k}{2}\right) \\
& =C\left(p_{1}, k\right)+C\left(p_{2}, k\right)+C\left(p_{3}, k\right)+C\left(p_{4}, k\right)-C\left(p_{1}+p_{4}, k\right)-C\left(p_{2}+p_{4}, k\right)-C\left(p_{3}+p_{4}, k\right)
\end{aligned}
$$

onde definimos

$$
C(p, k)=1-\cos \tilde{p} \cdot k
$$

Agindo com duas derivadas a direita e fazendo duas integrações por partes, pode-se mostrar que obtemos

$$
\begin{aligned}
& \Gamma_{\mu \nu \lambda \rho}^{\mathrm{st}}\left(p_{1}, p_{2}, p_{3}, p_{4}\right) \\
& \quad=i e \tilde{p}_{1, \rho} \Gamma_{\mu \nu \lambda}^{\mathrm{st}}\left(p_{1}+p_{4}, p_{2}, p_{3}\right)+\tilde{p}_{2, \rho} \Gamma_{\mu \nu \lambda}^{\mathrm{st}}\left(p_{1}, p_{2}+p_{4}, p_{3}\right)+\tilde{p}_{3, \rho} \Gamma_{\mu \nu \lambda}^{\mathrm{st}}\left(p_{1}, p_{2}, p_{3}+p_{4}\right)
\end{aligned}
$$

de onde segue a relação (3.36). 


\section{Apêndice F}

\section{F.1 Cálculo de $\mathcal{F}^{(0)}(x, k)$}

$$
\begin{aligned}
\mathcal{F}^{(0)}(x, k)= & \eta^{\mu \nu}\left\langle w_{\mu \nu}^{(0)}(x, k)\right\rangle \\
= & \int \frac{\mathrm{d}^{4} y}{(2 \pi)^{4}} e^{-i y \cdot k}\left\langle a_{\mu}\left(x_{+}\right) \star a^{\mu}\left(x_{-}\right)\right\rangle \\
= & \int \frac{\mathrm{d}^{4} y}{(2 \pi)^{4}} e^{-i y \cdot k} \sum_{s_{1}} \int \frac{\mathrm{d}^{3} k_{1}}{(2 \pi)^{3} 2 k_{1}^{0}} \epsilon_{1}^{\mu} \sum_{s_{2}} \int \frac{\mathrm{d}^{3} k_{2}}{(2 \pi)^{3} 2 k_{2}^{0}} \epsilon_{2 \mu} \\
\times & \left\langle\left[a_{1} \mathrm{e}^{-i k_{1} \cdot x_{+}}+a_{1}^{\dagger} \mathrm{e}^{i k_{1} \cdot x_{+}}\right] \star\left[a_{2} \mathrm{e}^{-i k_{2} \cdot x_{-}}+a_{2}^{\dagger} \mathrm{e}^{i k_{2} \cdot x_{-}}\right]\right\rangle \\
= & \int \frac{\mathrm{d}^{4} y}{(2 \pi)^{4}} e^{-i y \cdot k} \sum_{s_{1}} \int \frac{\mathrm{d}^{3} k_{1}}{(2 \pi)^{3} 2 k_{1}^{0}} \epsilon_{1}^{\mu} \sum_{s_{2}} \int \frac{\mathrm{d}^{3} k_{2}}{(2 \pi)^{3} 2 k_{2}^{0}} \epsilon_{2 \mu} \\
\times & {\left[\left\langle a_{1} a_{2}^{\dagger}\right\rangle \mathrm{e}^{-i k_{1} \cdot x_{+}} \star \mathrm{e}^{i k_{2} \cdot x_{-}}+\left\langle a_{1}^{\dagger} a_{2}\right\rangle \mathrm{e}^{i k_{1} \cdot x_{+}} \star \mathrm{e}^{-i k_{2} \cdot x_{-}}\right] } \\
= & \int \frac{\mathrm{d}^{4} y}{(2 \pi)^{4}} e^{-i y \cdot k} \int \frac{\mathrm{d}^{3} k_{1}}{(2 \pi)^{3} 2 k_{1}^{0}} \int \frac{\mathrm{d}^{3} k_{2}}{(2 \pi)^{3} 2 k_{2}^{0}} \sum_{s_{1}} \sum_{s_{2}} \epsilon_{1}^{\mu} \epsilon_{2 \mu} \\
\times & {\left[(2 \pi)^{3} 2 k_{1}^{0} \delta_{s_{1} s_{2}} \delta^{3}\left(k_{1}-k_{2}\right)\left(1+n_{B}\left(\left|k_{1}^{0}\right|\right)\right) \mathrm{e}^{-i k_{1} \cdot x_{+}} \star \mathrm{e}^{i k_{2} \cdot x_{-}}\right.} \\
& \left.+(2 \pi)^{3} 2 k_{1}^{0} \delta_{s_{1} s_{2}} \delta^{3}\left(k_{1}-k_{2}\right) n_{B}\left(\left|k_{1}^{0}\right|\right) \mathrm{e}^{i k_{1} \cdot x_{+}} \star \mathrm{e}^{-i k_{2} \cdot x_{-}}\right] \\
= & -\frac{2}{(2 \pi)^{3}} \delta\left(k^{2}\right)\left[n_{B}\left(\left|-k^{0}\right|\right) \theta\left(-k^{0}\right)+n_{B}\left(\left|k^{0}\right|\right) \theta\left(k^{0}\right)\right] \\
= & \int \frac{\mathrm{d}^{4} y}{(2 \pi)^{4}} e^{-i y \cdot k} \int \frac{\mathrm{d}^{3} k_{1}}{(2 \pi)^{3} 2 k_{1}^{0}}(-2)\left[\left(1+n_{B}\left(\left|k_{1}^{0}\right|\right)\right) \mathrm{e}^{-i k_{1} \cdot y}+n_{B}\left(\left|k_{1}^{0}\right|\right) \mathrm{e}^{i k_{1} \cdot y}\right] \\
& -2 \int \mathrm{d}^{4} k_{1} n^{3} \delta\left(k_{1}^{2}\right) \theta\left(k_{1}^{0}\right)\left[\left(1+k_{B}\left(\left|k_{1}^{0}\right|\right)\right) \delta\left(k+k_{1}\right)+n_{B}\left(\left|k_{1}^{0}\right|\right) \delta\left(k-k_{1}\right)\right] \\
& \\
& \\
& \\
& \\
&
\end{aligned}
$$


onde utilizamos a solução de ondas planas para o campo quântico $a_{\mu}(x)$,

$$
a^{\mu}(x)=\sum_{s_{1}} \int \frac{\mathrm{d}^{3} k_{1}}{(2 \pi)^{3} 2 k_{1}^{0}} \epsilon_{1}^{\mu}\left(a_{1} \mathrm{e}^{-i k_{1} \cdot x}+a_{1}^{\dagger} \mathrm{e}^{i k_{1} \cdot x}\right),
$$

onde $a_{1} \equiv a\left(k_{1}, s_{1}\right)$ e $a_{1}^{\dagger} \equiv a^{\dagger}\left(k_{1}, s_{1}\right)$ possuem "médias térmicas" dadas por

$$
\begin{aligned}
& \left\langle a_{1} a_{2}^{\dagger}\right\rangle=(2 \pi)^{3} 2 k_{1}^{0} \delta_{s_{1} s_{2}} \delta^{3}\left(k_{1}-k_{2}\right)\left(1+n_{B}\left(\left|k_{1}^{0}\right|\right)\right) \\
& \left\langle a_{1}^{\dagger} a_{2}\right\rangle=(2 \pi)^{3} 2 k_{1}^{0} \delta_{s_{1} s_{2}} \delta^{3}\left(k_{1}-k_{2}\right) n_{B}\left(\left|k_{1}^{0}\right|\right)
\end{aligned}
$$

e satisfazem a relação de comutação usual

$$
\left[a_{1}, a_{2}^{\dagger}\right]=(2 \pi)^{3} 2 k_{1}^{0} \delta_{s_{1} s_{2}} \delta^{3}\left(k_{1}-k_{2}\right)
$$

e $\epsilon_{1}^{\mu} \equiv \epsilon_{1}^{\mu}\left(k_{1}, s_{1}\right)$ é o vetor de polarização real que é transversal ao vetor de onda $k_{1}^{\mu}$, satisfazendo

$$
k_{1} \cdot \epsilon_{1}\left(k_{1}, s_{1}\right)=0 \quad \sum_{s_{1}=1,2} \epsilon_{1 \mu}\left(k_{1}, s_{1}\right) \epsilon_{1}^{\mu}\left(k_{1}, s_{1}\right)=-2 .
$$

Também utilizamos a representação integral em 4 dimensões da função delta de Dirac,

$$
\int \frac{\mathrm{d}^{4} y}{(2 \pi)^{4}} e^{-i y \cdot\left(k-k^{\prime}\right)}=\delta^{4}\left(k-k^{\prime}\right)
$$

e por último escrevemos a integral em $\mathrm{d}^{3} k_{1}$ na forma

$$
\int \frac{\mathrm{d}^{3} k_{1}}{(2 \pi)^{3} 2 k_{1}^{0}}=\int \frac{\mathrm{d}^{4} k_{1}}{(2 \pi)^{4}}(2 \pi) \delta\left(k_{1}^{2}\right) \theta\left(k_{0}^{1}\right) .
$$

O produto Moyal entre as exponenciais pode ser facilmente feita usando a propriedade (2.50), de maneira que temos

$$
\begin{aligned}
\mathrm{e}^{-i k_{1} \cdot x_{+}} \star \mathrm{e}^{i k_{1} \cdot x_{-}} & =\mathrm{e}^{-i k_{1} \cdot(x+y / 2)} \star \mathrm{e}^{i k_{1} \cdot(x-y / 2)} \\
& =\mathrm{e}^{-i k_{1} \cdot y} \mathrm{e}^{-i k_{1} \cdot x} \star \mathrm{e}^{i k_{1} \cdot x} \\
& =\mathrm{e}^{-i k_{1} \cdot y}
\end{aligned}
$$

lembrando que a não-comutatividade se dá apenas com a coordenada $x$, mas não com $y$.

\section{F.2 Cálculo de $\mathcal{F}^{(1)}(x, k)$}

$$
\mathcal{F}^{(1)}(x, k)=\frac{e}{2} \frac{1}{k \cdot \partial} \frac{\partial}{\partial k_{\sigma}} k^{\rho}\left[2 \bar{f}_{\rho \sigma} \mathcal{F}^{(0)}-2 \int \frac{\mathrm{d}^{4} y}{(2 \pi)^{4}} \mathrm{e}^{-i y \cdot k}\left\langle a_{\mu}\left(x_{+}\right) \star \bar{f}_{\rho \sigma} \star a^{\mu}\left(x_{-}\right)\right\rangle\right] .
$$


Vamos começar calculando a integral do segundo termo do lado direito,

$$
\begin{aligned}
& \int \frac{\mathrm{d}^{4} y}{(2 \pi)^{4}} \mathrm{e}^{-i y \cdot k}\left\langle a_{\mu}\left(x_{+}\right) \star \bar{f}_{\rho \sigma}(x) \star a^{\mu}\left(x_{-}\right)\right\rangle= \\
& \quad=\int \frac{\mathrm{d}^{4} y}{(2 \pi)^{4}} e^{-i y \cdot k} \sum_{s_{1}} \int \frac{\mathrm{d}^{3} k_{1}}{(2 \pi)^{3} 2 k_{1}^{0}} \epsilon_{1}^{\mu} \sum_{s_{2}} \int \frac{\mathrm{d}^{3} k_{2}}{(2 \pi)^{3} 2 k_{2}^{0}} \epsilon_{2 \mu} \\
& \quad \times\left\langle\left[a_{1} \mathrm{e}^{-i k_{1} \cdot x_{+}}+a_{1}^{\dagger} \mathrm{e}^{i k_{1} \cdot x_{+}}\right] \star \bar{f}_{\rho \sigma}(x) \star\left[a_{2} \mathrm{e}^{-i k_{2} \cdot x_{-}}+a_{2}^{\dagger} \mathrm{e}^{i k_{2} \cdot x_{-}}\right]\right\rangle \\
& \quad=\int \frac{\mathrm{d}^{4} y}{(2 \pi)^{4}} e^{-i y \cdot k} \sum_{s_{1}} \int \frac{\mathrm{d}^{3} k_{1}}{(2 \pi)^{3} 2 k_{1}^{0}} \epsilon_{1}^{\mu} \sum_{s_{2}} \int \frac{\mathrm{d}^{3} k_{2}}{(2 \pi)^{3} 2 k_{2}^{0}} \epsilon_{2 \mu} \\
& \quad \times\left[\left\langle a_{1} a_{2}^{\dagger}\right\rangle \mathrm{e}^{-i k_{1} \cdot x_{+}} \star \bar{f}_{\rho \sigma}(x) \star \mathrm{e}^{i k_{2} \cdot x_{-}}+\left\langle a_{1}^{\dagger} a_{2}\right\rangle \mathrm{e}^{i k_{1} \cdot x_{+}} \star \bar{f}_{\rho \sigma}(x) \star \mathrm{e}^{-i k_{2} \cdot x_{-}}\right] \\
& \left.=\int \frac{\mathrm{d}^{4} y}{(2 \pi)^{4}} e^{-i y \cdot k} \int \frac{\mathrm{d}^{3} k_{1}}{(2 \pi)^{3} 2 k_{1}^{0}}(-2)\left[\left(1+n_{B}\left(\left|k_{1}^{0}\right|\right)\right) \bar{f}_{\rho \sigma}\left(x-\theta k_{1}\right) \mathrm{e}^{-i k_{1} \cdot y}\right) \bar{f}_{\rho \sigma}\left(x+\theta k_{1}\right) \mathrm{e}^{i k_{1} \cdot y}\right] \\
& =-\frac{4}{(2 \pi)^{3}} n_{B}\left(\left|k^{0}\right|\right) \theta\left(k^{0}\right) \delta\left(k^{2}\right) \bar{f}_{\rho \sigma}(x+\theta k) \\
& =\mathcal{F}^{(0)} \bar{f}_{\rho \sigma}(x+\theta k)
\end{aligned}
$$

onde usamos a propriedade (2.50) para fazer o produto Moyal,

$$
\begin{aligned}
\mathrm{e}^{-i k_{1} \cdot x_{+}} \star \bar{f}_{\rho \sigma}(x) \star \mathrm{e}^{i k_{1} \cdot x_{-}} & =\mathrm{e}^{-i k_{1} \cdot(x+y / 2)} \star \bar{f}_{\rho \sigma}(x) \star \mathrm{e}^{i k_{1} \cdot(x-y / 2)} \\
& =\mathrm{e}^{-i k_{1} \cdot y} \mathrm{e}^{-i k_{1} \cdot x} \star \bar{f}_{\rho \sigma}(x) \star \mathrm{e}^{i k_{1} \cdot x} \\
& =\mathrm{e}^{-i k_{1} \cdot y} \bar{f}_{\rho \sigma}\left(x-\theta k_{1}\right)
\end{aligned}
$$

Desta forma, $\mathcal{F}^{(1)}$ pode ser escrito como

$$
\mathcal{F}^{(1)}(x, k)=-\frac{4 e}{(2 \pi)^{3}} \frac{1}{k \cdot \partial} \frac{\partial}{\partial k_{\sigma}} k^{\rho}\left[n_{B}\left(\left|k^{0}\right|\right) \theta\left(k^{0}\right) \delta\left(k^{2}\right)\left(\bar{f}_{\rho \sigma}(x)-\bar{f}_{\rho \sigma}(x+\theta k)\right)\right] .
$$




\section{F.3 Cálculo de $\mathcal{F}^{(2)}(x, k)$}

$$
\begin{aligned}
\mathcal{F}^{(2)}(x, k)= & \frac{1}{k \cdot \partial}\left\{i e\left[k \cdot \bar{A}(x), \mathcal{F}^{(1)}\right] \mathrm{MB}\right. \\
& +\frac{e}{2} \frac{\partial}{\partial k_{\sigma}} k^{\rho}\left[\bar{f}_{\rho \sigma}(x) \star \mathcal{F}^{(1)}(x, k)+\mathcal{F}^{(1)}(x, k) \star \bar{f}_{\rho \sigma}(x)\right. \\
& -i e\left[\bar{A}_{\rho}(x), \bar{A}_{\sigma}(x)\right]_{\mathrm{MB}} \star \mathcal{F}^{(0)}-i e \mathcal{F}^{(0)} \star\left[\bar{A}_{\rho}(x), \bar{A}_{\sigma}(x)\right]_{\mathrm{MB}} \\
& -2 \int \frac{\mathrm{d}^{4} y}{(2 \pi)^{4}} \mathrm{e}^{-i y \cdot k}\left\langle G_{\mu}\left(x_{+}\right) \star\left(-i e\left[\bar{A}_{\rho}, \bar{A}_{\sigma}\right]_{\mathrm{MB}}\right) \star G^{\mu}\left(x_{-}\right)\right\rangle \\
& \left.\left.-2 \int \frac{\mathrm{d}^{4} y}{(2 \pi)^{4}} \mathrm{e}^{-i y \cdot k}\left\langle G_{\mu}\left(x_{+}\right) \star \bar{f}_{\rho \sigma}(x) \star G^{\mu}\left(x_{-}\right)\right\rangle\right]\right\} .
\end{aligned}
$$

As contribuições da primeira, segunda e terceira linhas podem ser obtidas diretamente, pois $\mathcal{F}^{(0)}$ e $\mathcal{F}^{(1)}$ já estão determinados. A quarta linha está calculada em (5.82). O cálculo da última linha pode ser feito como segue:

$$
\begin{aligned}
& \int \frac{\mathrm{d}^{4} y}{(2 \pi)^{4}} \mathrm{e}^{-i y \cdot k}\left\langle G_{\mu}\left(x_{+}\right) \star \bar{f}_{\rho \sigma}(x) \star G^{\mu}\left(x_{-}\right)\right\rangle= \\
& =\int \frac{\mathrm{d}^{4} y}{(2 \pi)^{4}} \mathrm{e}^{-i y \cdot k}\left\langle a_{\mu}\left(x_{+}\right) \star \bar{f}_{\rho \sigma}(x) \star a^{\mu}\left(x_{-}\right)\right\rangle \\
& =\int \frac{\mathrm{d}^{4} y}{(2 \pi)^{4}} e^{-i y \cdot k} \sum_{s_{1}} \int \frac{\mathrm{d}^{3} k_{1}}{(2 \pi)^{3} 2 k_{1}^{0}} \epsilon_{1}^{\mu} \sum_{s_{2}} \int \frac{\mathrm{d}^{3} k_{2}}{(2 \pi)^{3} 2 k_{2}^{0}} \epsilon_{2 \mu} \\
& \times\left\langle\left[a_{1} \mathrm{e}^{-i k_{1} \cdot X_{+}}+a_{1}^{\dagger} \mathrm{e}^{i k_{1} \cdot X_{+}}\right] \star \bar{f}_{\rho \sigma}(x) \star\left[a_{2} \mathrm{e}^{-i k_{2} \cdot X_{-}}+a_{2}^{\dagger} \mathrm{e}^{i k_{2} \cdot X_{-}}\right]\right\rangle \\
& =\int \frac{\mathrm{d}^{4} y}{(2 \pi)^{4}} e^{-i y \cdot k} \sum_{s_{1}} \int \frac{\mathrm{d}^{3} k_{1}}{(2 \pi)^{3} 2 k_{1}^{0}} \epsilon_{1}^{\mu} \sum_{s_{2}} \int \frac{\mathrm{d}^{3} k_{2}}{(2 \pi)^{3} 2 k_{2}^{0}} \epsilon_{2 \mu} \\
& \times\left[\left\langle a_{1} a_{2}^{\dagger}\right\rangle \mathrm{e}^{-i k_{1} \cdot X_{+}} \star \bar{f}_{\rho \sigma}(x) \star \mathrm{e}^{i k_{2} \cdot X_{-}}+\left\langle a_{1}^{\dagger} a_{2}\right\rangle \mathrm{e}^{i k_{1} \cdot X_{+}} \star \bar{f}_{\rho \sigma}(x) \star \mathrm{e}^{-i k_{2} \cdot X_{-}}\right] \\
& =\int \frac{\mathrm{d}^{4} y}{(2 \pi)^{4}} e^{-i y \cdot k} \int \frac{\mathrm{d}^{3} k_{1}}{(2 \pi)^{3} 2 k_{1}^{0}}(-2)\left[\left(1+n_{B}\left(\left|k_{1}^{0}\right|\right)\right) \mathrm{e}^{-i k_{1} \cdot y} \mathrm{e}^{-i k_{1} \cdot X} \star \bar{f}_{\rho \sigma}(x) \star \mathrm{e}^{i k_{1} \cdot X}\right. \\
& \left.+n_{B}\left(\left|k_{1}^{0}\right|\right) \mathrm{e}^{i k_{1} \cdot y} \mathrm{e}^{i k_{1} \cdot X} \star \bar{f}_{\rho \sigma}(x) \star \mathrm{e}^{-i k_{1} \cdot X}\right] \\
& =\int \frac{\mathrm{d}^{4} y}{(2 \pi)^{4}} e^{-i y \cdot k} \int \frac{\mathrm{d}^{3} k_{1}}{(2 \pi)^{3} 2 k_{1}^{0}}(-2)\left[\left.\left(1+n_{B}\left(\left|k_{1}^{0}\right|\right)\right) \mathrm{e}^{-i k_{1} \cdot y}\left(\mathrm{e}_{*}^{-\tilde{k}_{1} \cdot \tilde{D}} \bar{f}_{\rho \sigma}(x)\right)\right|^{(e)}\right. \\
& \left.+\left.n_{B}\left(\left|k_{1}^{0}\right|\right) \mathrm{e}^{i k_{1} \cdot y}\left(\mathrm{e}_{*}^{\tilde{k}_{1} \cdot \tilde{D}} \bar{f}_{\rho \sigma}(x)\right)\right|^{(e)}\right] \\
& =-\left.\frac{4}{(2 \pi)^{3}} n_{B}\left(\left|k^{0}\right|\right) \theta\left(k^{0}\right) \delta\left(k^{2}\right)\left(\mathrm{e}_{*}^{\tilde{k} \cdot \tilde{D}} \bar{f}_{\rho \sigma}(x)\right)\right|^{(e)}
\end{aligned}
$$


onde usamos a definição $X_{ \pm}^{\mu}=x_{ \pm}^{\mu}+e \theta^{\mu \nu} \tilde{A}_{\nu}(x)$ e a propriedade,

$$
\begin{aligned}
\mathrm{e}^{-i k \cdot X} \star \bar{f}_{\rho \sigma}(x) \star \mathrm{e}^{i k \cdot X} & =\mathrm{e}^{-i(k \cdot x+e k \times \tilde{A})} \star \bar{f}_{\rho \sigma}(x) \star \mathrm{e}^{i(k \cdot x+e k \times \tilde{A})} \\
& =\mathrm{e}_{*}^{-\tilde{k} \cdot \tilde{D}} \bar{f}_{\rho \sigma}(x)
\end{aligned}
$$

que pode ser obtida expandindo-se o produto Moyal e reagrupando-se os termos do lado esquerdo. O sobrescrito $\left.\right|^{(e)}$ significa que devemos tomar termos lineares em $e$ desta expressão. Para concluir, precisamos da propriedade

$$
\begin{aligned}
\mathrm{e}^{i(k \cdot x+e k \times \tilde{A})} \star & \bar{f}_{\rho \sigma}(x) \star \mathrm{e}^{-\left.i(k \cdot x+e k \times \tilde{A})\right|^{(e)}}=\left.\left(\mathrm{e}^{\tilde{k} \cdot \tilde{D}} \bar{f}_{\rho \sigma}(x)\right)\right|^{(e)} \\
& =-i e \sum_{n=1}^{\infty} \frac{1}{n !}\left[\left\{(\tilde{k} \cdot \partial)^{n-1}(\tilde{k} \cdot \tilde{A})+(\tilde{k} \cdot \partial)^{n-2}(\tilde{k} \cdot \tilde{A})(\tilde{k} \cdot \partial)+\cdots+(\tilde{k} \cdot \tilde{A})(\tilde{k} \cdot \partial)^{n-1}\right\}, \bar{f}_{\rho \sigma}\right]_{\mathrm{MB}}
\end{aligned}
$$

Feitas estas considerações, a expressão para $\mathcal{F}^{(2)}$ será

$$
\begin{aligned}
\mathcal{F}^{(2)}(x, k)= & i e \frac{1}{k \cdot \partial}\left[k \cdot \bar{A}(x), \mathcal{F}^{(1)}(x, k)\right]_{\mathrm{MB}} \\
& -\frac{4 i e^{2}}{(2 \pi)^{3}} \frac{1}{k \cdot \partial} \frac{\partial}{\partial k_{\sigma}} k^{\rho} \delta\left(k^{2}\right) n_{B}\left(\left|k^{0}\right|\right)\left\{\left[\bar{A}_{\rho}(x), \bar{A}_{\sigma}(x)\right]_{\mathrm{MB}}-\left[\bar{A}_{\rho}(x+\theta k), \bar{A}_{\sigma}(x+\theta k)\right]_{\mathrm{MB}}\right. \\
& \left.+\left[\frac{1}{k \cdot \partial} k \cdot(\bar{A}(x)-\bar{A}(x+\theta k)), \bar{f}_{\rho \sigma}(x+\theta k)\right]_{\mathrm{MB}}\right\}
\end{aligned}
$$

No cálculo explícito de $J_{\mu}^{(3)}$ é mais conveniente calcular o termo (F.17) no espaço dos momentos. Embora um pouco trabalhoso, pode-se mostrar que o lado direito de (F.17) pode ser escrito como

$$
\begin{aligned}
& -i e \int \mathrm{d}^{4} p_{2} \mathrm{~d}^{4} p_{3} \delta\left(p_{1}+p_{2}+p_{3}\right)(-2 i) \sin \left(\frac{p_{2} \times p_{3}}{2}\right)\left(\tilde{k} \cdot \tilde{A}\left(p_{2}\right)\right) \bar{f}_{\rho \sigma}\left(k_{3}\right) \\
& \times \sum_{n=1}^{\infty} \frac{1}{n !}\left\{\left(i \tilde{k} \cdot p_{1}\right)^{n-1}+\left(i \tilde{k} \cdot p_{1}\right)^{n-2}\left(-i \tilde{k} \cdot p_{3}\right)+\cdots+\left(-i \tilde{k} \cdot p_{3}\right)^{n-1}\right\} \\
& =-2 e \int \mathrm{d}^{4} p_{2} \mathrm{~d}^{4} p_{3} \delta\left(p_{1}+p_{2}+p_{3}\right) \sin \left(\frac{p_{1} \times p_{2}}{2}\right)\left(\tilde{k} \cdot \tilde{A}\left(p_{2}\right)\right) \bar{f}_{\rho \sigma}\left(p_{3}\right) \\
& \quad \times \sum_{n=1}^{\infty} \frac{1}{n !} \frac{\left(i \tilde{k} \cdot p_{1}\right)^{n}-\left(-i \tilde{k} \cdot p_{3}\right)^{n}}{\left(i \tilde{k} \cdot p_{1}\right)-\left(-i \tilde{k} \cdot p_{3}\right)} \\
& =-2 i e \int \mathrm{d}^{4} p_{2} \mathrm{~d}^{4} p_{3} \delta\left(p_{1}+p_{2}+p_{3}\right) \sin \left(\frac{p_{1} \times p_{2}}{2}\right) \frac{\tilde{k} \cdot \tilde{A}\left(p_{2}\right)}{\tilde{k} \cdot p_{2}} \bar{f}_{\rho \sigma}\left(p_{3}\right)\left(e^{i \tilde{k} \cdot p_{1}}-e^{-i \tilde{k} \cdot p_{3}}\right),
\end{aligned}
$$

onde $\bar{f}_{\rho \sigma}\left(p_{3}\right)=-i\left(p_{3 \rho} \bar{A}_{\sigma}\left(p_{3}\right)-p_{3 \sigma} \bar{A}_{\rho}\left(p_{3}\right)\right)$. 


\section{Apêndice G}

\section{G.1 Quantização da QED não-comutativa em um campo de fundo}

Sabemos que a quantização canônica de teorias de gauge não-abelianas é altamente não trivial [67], por isso outros métodos foram desenvolvidos, como por exemplo a quantização por integrais de trajetória e mais recentemente a quantização estocástica. A dificuldade está no fato de que não é fácil encontrar uma base completa para as equações do campo quântico, que são equações não-lineares. Um caso onde a quantização canônica pode ser feita com sucesso é quando o campo de fundo é constante [67]. Entretanto, existem situações físicas onde o campo de fundo pode ser considerado como sendo fraco e com variações mais lentas que o campo quântico, de forma que o método da quantização canônica em um campo de fundo pode ser realizado com sucesso. Vamos então aplicar este método à QED não-comutativa. Isto se justifica na aproximação de altas temperaturas (limite HTL) que estamos adotando aqui, pois no nosso caso o plasma quente que estamos estudando é um exemplo onde os campos externos (campos de fundo) são fracos com relação aos campos que descrevem as partículas em um loop interno (campos quânticos), de forma que é satisfeita a relação $p<<k \sim T$, onde $p$ representa um momento externo típico e $k$ representa o momento interno ao loop.

A quantização não é afetada pela presença de campos de férmions, de maneira que vamos nos restringir ao caso da teoria de gauge $U(1)$ não-comutaiva ${ }^{1}$. A ação da teoria é descrita por

$$
S[A]=\int \mathrm{d}^{4} x\left(-\frac{1}{4} F_{\mu \nu} \star F^{\mu \nu}\right)
$$

onde

$$
F_{\mu \nu}(A)=\partial_{\mu} A_{\nu}-\partial_{\nu} A_{\mu}-i e\left[A_{\mu}, A_{\nu}\right]_{\mathrm{MB}} .
$$

Fazemos então a decomposição do campo de gauge em uma parte de campo de fundo $\bar{A}_{\mu}$, e uma parte quântica $a_{\mu}$,

$$
A_{\mu}=\bar{A}_{\mu}+a_{\mu}, \quad\left\langle a_{\mu}\right\rangle=0
$$

onde $\left\langle a_{\mu}\right\rangle$ representa o valor esperado do campo quântico em um dado estado e $\bar{A}_{\mu}$ satisfaz

$$
D_{\mu}(\bar{A}) F^{\mu \nu}(\bar{A}) \equiv \bar{D}_{\mu} \bar{F}^{\mu \nu}=\partial_{\mu} \bar{F}^{\mu \nu}-i e\left[\bar{A}_{\mu}, \bar{F}^{\mu \nu}\right]_{\mathrm{MB}}=0 .
$$

\footnotetext{
${ }^{1}$ Que chamamos anteriormente de QED pura não-comutativa, ou ainda teoria de Maxwell não-comutativa.
} 
Segue então que podemos escrever $F^{\mu \nu}$ como

$$
F_{\mu \nu}=\bar{F}_{\mu \nu}+\bar{D}_{\mu} a_{\nu}-\bar{D}_{\nu} a_{\mu}-i e\left[a_{\mu}, a_{\nu}\right]_{\mathrm{MB}},
$$

e a ação (G.1) pode ser expandida como

$$
\begin{array}{r}
S[\bar{A}+a]=\int \mathrm{d}^{4} x[- \\
-\frac{1}{4} \bar{F}_{\mu \nu} \star \bar{F}^{\mu \nu}-\frac{1}{2}\left(\bar{D}^{\mu} a^{\nu}-\bar{D}^{\nu} a^{\mu}\right) \star \bar{D}_{\mu} a_{\nu}+i e \bar{F}^{\mu \nu} \star a_{\mu} \star a_{\nu} \\
\left.+i e\left(\bar{D}^{\mu} a^{\nu}-\bar{D}^{\nu} a^{\mu}\right) \star a_{\mu} \star a_{\nu}+\frac{e^{2}}{2}\left[a^{\mu}, a^{\nu}\right]_{\mathrm{MB}} \star a_{\mu} \star a_{\nu}\right] .
\end{array}
$$

A vantagem do método do campo de fundo é que a invariância de gauge original da teoria pela transformação

$$
\delta A_{\mu}(x)=\partial_{\mu} \epsilon(x)-i e\left[A_{\mu}(x), \epsilon(x)\right]_{\mathrm{MB}}
$$

pode ser vista de duas maneiras. Podemos pensá-la como uma invariância de gauge quântica pela transformação

$$
\begin{aligned}
\delta \bar{A}_{\mu}(x) & =0, \\
\delta a_{\mu}(x) & =\partial_{\mu} \epsilon(x)-i e\left[\bar{A}_{\mu}(x)+a_{\mu}(x), \epsilon(x)\right]_{\mathrm{MB}},
\end{aligned}
$$

ou podemos pensá-la como uma invariância de gauge do campo de fundo por

$$
\begin{aligned}
\delta \bar{A}_{\mu}(x) & =\partial_{\mu} \epsilon(x)-i e\left[\bar{A}_{\mu}(x), \epsilon(x)\right]_{\mathrm{MB}}, \\
\delta a_{\mu}(x) & =-i e\left[a_{\mu}(x), \epsilon(x)\right]_{\mathrm{MB}} .
\end{aligned}
$$

Ou seja, por uma transformação de gauge quântica, o campo de fundo permanece inerte enquanto o campo quântico se transforma como um campo de gauge. Por outro lado, por uma transformação de gauge do campo de fundo, o campo quântico se transforma na sua representação adjunta. Podemos então tirar vantagem disso e adicionar à ação um termo fixador de gauge e um termo de ghost (fantasma)

$$
S_{\mathrm{GF}}+S_{\text {ghost }}=\int \mathrm{d}^{4} x\left[-\frac{1}{2 \xi}(\bar{D} \cdot a) \star(\bar{D} \cdot a)+\bar{D}^{\mu} \bar{c} \star\left(\partial_{\mu} c-i e\left[\bar{A}_{\mu}+a_{\mu}, c\right]_{\mathrm{MB}}\right)\right],
$$

que quebra a invariância de gauge quântica (G.8), mas permanece invariante pela transformação de gauge do campo de fundo (G.9), com os campos de ghosts transformando-se na representação adjunta. Desta forma, cálculos realizados com este termo fixador de gauge leva a resultados manifestamente invariantes por transformações de gauge do campo de fundo.

A parte da ação total que é responsável pelos resultados em 1-loop é aquela que é quadrática nos campos quânticos, de forma que podemos nos restringir a

$$
\begin{aligned}
S_{q}=\int \mathrm{d}^{4} x & {\left[-\frac{1}{2}\left(\bar{D}^{\mu} a^{\nu}-\bar{D}^{\nu} a^{\mu}\right) \star \bar{D}_{\mu} a_{\nu}+i e \bar{F}^{\mu \nu} \star a_{\mu} \star a_{\nu}\right.} \\
& \left.-\frac{1}{2 \xi}(\bar{D} \cdot a) \star(\bar{D} \cdot a)+\bar{D}^{\mu} \bar{c} \star \bar{D}_{\mu} c\right] .
\end{aligned}
$$


Aqui $\xi$ representa o parâmetro fixador de gauge e no limite $\xi \rightarrow 0$ devemos ter a seguinte condição de gauge:

$$
\bar{D} \cdot a=\bar{D}_{\mu} a^{\mu}=\partial_{\mu} a^{\mu}-i e\left[\bar{A}_{\mu}, a^{\mu}\right]_{\mathrm{MB}}=0 .
$$

Neste gauge, a equação de movimento que segue de

$$
\frac{\partial \mathscr{L}_{q}}{\partial a_{\mu}}-\bar{D}_{\nu} \frac{\partial \mathscr{L}_{q}}{\partial \bar{D}_{\nu} a_{\mu}}=0
$$

para o campo quântico é

$$
\bar{D}^{2} a^{\mu}=\bar{D}^{\nu} \bar{D}^{\mu} a_{\nu}+i e\left[\bar{F}^{\mu \nu}, a_{\nu}\right]_{\mathrm{MB}} .
$$

Usando a propriedade

$$
\left(\bar{D}^{\mu} \bar{D}^{\nu}-\bar{D}^{\nu} \bar{D}^{\mu}\right) a_{\nu}=-i e\left[\bar{F}^{\mu \nu}, a_{\nu}\right]_{\mathrm{MB}}
$$

podemos reescrever a equação de movimento (G.14) simplesmente como

$$
\bar{D}^{2} a^{\mu}=2 i e\left[\bar{F}^{\mu \nu}, a_{\nu}\right]_{\mathrm{MB}} .
$$

A solução desta equação é muito difícil, mas no limite de altas temperaturas em que estamos interessados ela pode ser resolvida. Em primeiro lugar lembre-se que o momento externo $p$ está associado ao campo de fundo $\bar{A}$ e o momento interno ao loop, representado por $k$, está associado ao campo quântico $a$. Desta forma, uma derivada agindo em $\bar{A}$ é proporcional a $p$, ou seja $\partial \bar{A} \sim p \bar{A}$, e quando agindo em $a$ é proporcional a $k$, ou seja $\partial a \sim k a$. É fácil ver então que todos os termos do lado direito de (G.16) são proporcionais a ep $\bar{A} a$ e $e^{2} \bar{A}^{2} a$, enquanto que do lado esquerdo temos termos proporcionais a $\bar{A} k a$. O lado direito representa a variação do campo de fundo $\bar{A}$, enquanto o lado esquerdo representa a variação do campo quântico $a$. Como dissemos anteriormente, o método da quantização em um campo de fundo se aplica no caso em que o campo de fundo é fraco ou varia lentamente com relação ao campo quântico, que é o nosso caso, pois temos $p<<k$. Por isso podemos fazer a aproximação $p \bar{A} a<<\bar{A} k a$, e despresar os termos de ordem $e^{2} \bar{A}^{2}$, de forma que o lado direito de (G.16) não contribui para nossa aproximação, e ficamos com

$$
\bar{D}^{2} a^{\mu}=0
$$

onde o operador D'Alembertiano na aproximação considerada é dado simplesmente por

$$
\bar{D}^{2} \simeq \partial^{2}-2 i e\left[\bar{A}_{\sigma}, \partial^{\sigma}\right]_{\mathrm{MB}}
$$

Agora vamos encontrar uma base que seja solução da equação (G.17). Sabemos que, na ausência de qualquer campo de fundo, uma solução para o operador D'Alembertiano são as ondas planas, da forma 
$\mathrm{e}^{i k x}$. Vamos então mostrar que, no nosso caso não-comutativo na presença de um campo de fundo $\bar{A}$ e nas aproximações acima, a solução até ordem $e$ da equação ${ }^{2}$

$$
\bar{D}^{2} b=0
$$

é da forma

$$
b=\mathrm{e}^{i k \cdot X},
$$

onde

$$
X^{\mu}=x^{\mu}+e \theta^{\mu \nu} \tilde{A}_{\nu}(x), \quad \tilde{A}_{\nu}(x)=\bar{A}_{\nu}(x)+\frac{1}{k \cdot \bar{D}} \bar{F}_{\nu \alpha} k^{\alpha}
$$

Nossa demonstração será feita em primeira ordem para o parâmetro $\theta$, mas pode-se mostrar que o resultado é válido para o caso geral, conforme [71].

Em primeiro lugar, vamos expandir $\tilde{A}_{\mu}(x)$ em potências de $e$, e tomar somente o primeiro termo, de ordem zero em $e$,

$$
\begin{aligned}
\tilde{A}_{\mu}(x) & =\bar{A}_{\mu}+\frac{1}{k \cdot \bar{D}} \bar{F}_{\mu \alpha} k^{\alpha} \\
& =\bar{A}_{\mu}+\frac{1}{k \cdot \bar{D}}\left(\partial_{\mu} \tilde{A}_{\alpha}-\partial_{\alpha} \tilde{A}_{\mu}-i e\left[\tilde{A}_{\mu}, \tilde{A}_{\alpha}\right]_{\mathrm{MB}}\right) k^{\alpha} \\
& =\bar{A}_{\mu}-\frac{1}{k \cdot \bar{D}}\left(\bar{D}_{\alpha} \tilde{A}_{\mu}\right) k^{\alpha}+\frac{\partial_{\mu} \tilde{A}_{\alpha}}{k \cdot \bar{D}} k^{\alpha} \\
& =\frac{\partial_{\mu}(\tilde{A} \cdot k)}{k \cdot \bar{D}} \\
& \simeq \frac{\partial_{\mu}(\tilde{A} \cdot k)}{k \cdot \partial} .
\end{aligned}
$$

Para ordem mais baixa em $\theta$, podemos escrever ${ }^{3}$

$$
\mathrm{e}^{i k \cdot X} \simeq \mathrm{e}^{i k \cdot x} \mathrm{e}^{i e k_{\alpha} \theta^{\alpha \beta} \tilde{A}_{\beta}}
$$

de forma que, até termos lineares em $\theta$, temos

$$
\partial^{2} \mathrm{e}^{i k \cdot X} \simeq-2 e k_{\alpha} \theta^{\alpha \beta} \partial_{\beta}(k \cdot \tilde{A}) \mathrm{e}^{i k \cdot X}+i e k_{\alpha} \theta^{\alpha \beta} \frac{\partial^{2}}{k \cdot \partial} \partial_{\beta}(k \cdot \tilde{A}) \mathrm{e}^{i k \cdot X}+\mathcal{O}\left(\theta^{2}\right),
$$

onde usamos o resultado (G.22) e o fato que $k^{2}=0$. Mas o segundo termo do lado direito contém um fator do tipo $\partial^{2} / k \cdot \partial \sim p^{2} / k \cdot p$ com relação ao primeiro, e no limite $p<<k$ temos $p^{2} / k \cdot p<<1$, portanto

\footnotetext{
${ }^{2}$ Lembre-se que estamos interessados nas contribuições de ordem $e$ vindas do campo quântico $a_{\mu}$.

${ }^{3}$ No caso geral, devido à não-comutatividade dos dois expoentes, a decomposição deve ser feita usando-se a fórmula de BakerCampbell-Hausdorff $\mathrm{e}^{A} \mathrm{e}^{B}=\mathrm{e}^{A+B+1 / 2[A, B]+1 / 12[A,[A, B]]-1 / 12[B,[B, A]]+\cdots}$.
} 
este termo pode ser despresado com relação ao primeiro. Portanto

$$
\partial^{2} \mathrm{e}^{i k \cdot X} \simeq-2 e k_{\alpha} \theta^{\alpha \beta} \partial_{\beta}(k \cdot \tilde{A}) \mathrm{e}^{i k \cdot X}+\mathcal{O}\left(\theta^{2}\right)
$$

Temos também que, até ordem $\theta$,

$$
\begin{aligned}
-2 i e\left[\tilde{A}_{\sigma}, \partial^{\sigma} \mathrm{e}^{i k \cdot X}\right]_{\mathrm{MB}} & =-2 i e(2 i) \sin \left(\frac{1}{2} \theta^{\alpha \beta}\left(\partial_{\alpha} \tilde{A}_{\sigma}\right)\left(\partial_{\beta} \partial^{\sigma} \mathrm{e}^{i k \cdot X}\right)\right) \\
& \simeq 2 e k_{\alpha} \theta^{\alpha \beta} \partial_{\beta}(k \cdot \tilde{A}) \mathrm{e}^{i k \cdot X}+\mathcal{O}\left(\theta^{2}\right)
\end{aligned}
$$

onde usamos a propriedade (2.44) para o comutador Moyal entre duas funções e tomamos apenas o primeiro termo da expansão da função seno, que já é linear em $\theta$. Portanto temos

$$
\begin{aligned}
\bar{D}^{2} \mathrm{e}^{i k \cdot X} & \simeq \partial^{2} \mathrm{e}^{i k \cdot X}-2 i e\left[\bar{A}_{\sigma}, \partial^{\sigma} \mathrm{e}^{i k \cdot X}\right]_{\mathrm{MB}} \\
& =0+\mathcal{O}\left(\theta^{2}\right)
\end{aligned}
$$

e assim fica demonstrado que até termos de ordem $\theta$ a solução de (G.19) é dada por (G.20).

Portanto, a solução quântica para o campo $a_{\mu}$ que satisfaça (G.17) deve ser da forma

$$
a^{\mu}(x)=\sum_{s} \int \frac{\mathrm{d}^{3} k}{(2 \pi)^{3} 2 k^{0}} \epsilon^{\mu}\left(a \mathrm{e}^{-i k \cdot X}+a^{\dagger} \mathrm{e}^{i k \cdot X}\right)
$$

que chamamos anteriormente de solução quântica covariantizada, já que ela se transforma covariantemente por transformações de gauge. 


\section{Referências Bibliográficas}

[1] C. Itzykson and J. B. Zuber, Quantum Field Theory, McGraw-Hill, Singapore, 1985.

[2] L. H. Ryder, Quantum Field Theory (2nd ed.), Cambridge University Press, Cambridge, 1996.

[3] D. Bailin e A. Love, Introduction to Gauge field Theory, (Revised edition), University of Sussex Press, 1993.

[4] F. Mandl and G. Shaw, Quantum Field Theory, John Wiley e Sons Ltd, New York, 1984.

[5] M. E. Peskin and D. V. Shroeder, An Introduction to Quantum Field Theory, Addison-Wesley Publishing Company, 1995.

[6] M. O. C. Gomes, Teoria Quântica dos Campos, EDUSP, 2002.

[7] J. M. Jauch and F. Rohnlich, The Theory of Photons and Electrons (2nd ed.), Springer-Verlag, New York, 1976.

[8] J. D. Bjorken and S. D. Drell, Relativistic Quantum Mechanics, McGraw-Hill, New York, 1964.

[9] D. Griffiths, Introduction to Elementary Particles, John Wiley e Sons Ltd, New York, 1987.

[10] A. Das, Field Theory: A Path Integral Approach, World Sci. Pub. Co., Singapure, 1993.

[11] J. I. Kapusta, Finite Temperature Field Theory, Cambridge University Press, Cambridge, England, 1989.

[12] M. L. Bellac, Thermal Field Theory, Cambridge University Press, Cambridge, England, 1996.

[13] A. Das, Finite Temperature Field Theory, World Scientific, NY, 1997.

[14] C. W. Bernard, Phys. Rev. D 9, 12 (1974).

[15] J. I. Kapusta and P. V. Landshoff, J. Phys. G: Nucl. Part. Phys. 15, 267 (1989).

[16] E. S. Fradkin, Proc. Lebedev Inst. 29, 6 (1965).

[17] H. A. Weldon, Phys. Rev. D 26, 1394 (1982); Phys. Rev. D 26, 2789 (1982).

[18] H. S. Snyder, Phys. Rev. 71, 38 (1947). 
[19] M. Chaichian, M. M. Sheikh-Jabbari and A. Tureanu, Phys. Rev. Lett. 86, 2716 (2001).

[20] S. M. Carroll, J. A. Harvey, V. A. Kostelecky, C. D. Lane and T. Okamoto, Phys. Rev. Lett. 87, 141601 (2001).

[21] L. D. Landau and E. M. Lifshitz, Quantum Mechanics: The Non-Relativistic Theory, ButterworthHeinemann, 1981.

[22] L. E. Ballentine, Quantum Mechanics, Prentice Hall, 1990; S. Gasiorowicz, Quantum Physics (2nd edition), Wiley, 1996; E. Merzbacher, Quantum Mechanics (3rd edition), Wiley, 1998; J. Schwinger, Quantum Mechanics, Springer, 2001.

[23] R. Jackiw, G. V. Dunne and C. A. Trugenberger, Phys. Rev. D 41, 661 (1990); G. V. Dunne and R. Jackiw, Nucl. Phys. (Proc. Suppl.) 33 C, 114 (1993).

[24] R. Jackiw, S. Y. Pi and A. Polychronakos, Ann. Phys. (NY) 301, 157 (2002).

[25] R. Jackiw, Nucl. Phys. (Proc. Suppl.) 108, 30, (2002); Ann. Henri Poincare 4 (Suppl. 2), 913, (2003).

[26] R. Jackiw, hep-th/9306075 (1993).

[27] P. A. M. Dirac, Lectures on Quantum Mechanics, Yeshiva University, New York, 1964.

[28] M. Henneaux and C. Teitelboim, Quantization of Gauge Systems, Princenton University, Princeton, 1992.

[29] M. Chaichian, M. M. Sheikh-Jabbari and A. Tureanu, hep-th/0511323 (2005).

[30] E. Harikumar and V. O. Rivelles, Class. Quant. Grav. 23, 7551 (2006).

[31] S. Calmet and A. Kobakhidze, Phys. Rev. D 72, 045010 (2005).

[32] F. T. Brandt and M. R. Elias-Filho, Phys. Rev. D 74, 067704 (2006).

[33] B. A. Charneski, "Três diferentes abordagens não-comutativas aos modelos de Gross-Neveu e sigma não-linear na expansão perturbativa 1/N”, Dissertação de mestrado, IF-USP, São Paulo (2006).

[34] A. Connes, Noncommutative Geometry, Academic Press, 1994.

[35] N. Seiberg and E. Witten, JHEP 9909, 032 (1999).

[36] M. R. Douglas and N. A. Nekrasov, Rev. Mod. Phys. 73, 977 (2002).

[37] R. J. Szabo, Phys. Rep. 378, 207 (2003).

[38] H. O. Girotti, hep-th/0301237 (2003).

[39] M. Gomes, 11th Jorge Andre Swieca Summer School on Particle and Fields, 251, (2001). 
[40] S. Doplicher, K. Fredenhagen and J. E. Roberts, hep-th/0303037 (2003).

[41] F. T. Brandt, A. Das and J. Frenkel, Phys. Rev. D 65, 085017 (2002).

[42] A. F. Ferrari, M. Gomes, A. Yu Petrov and A. J. da Silva, Phys. Lett. B 638, 275 (2006).

[43] B. Charneski, A. F. Ferrari and M. Gomes, J. Phys. A 40, 3633 (2007).

[44] R. J. Szabo, hep-th/0701224 (2007).

[45] A. Das and J. Frenkel, Phys. Rev. D 69, 065017 (2004).

[46] F. T. Brandt, J. Frenkel and D. G. C. McKeon, Phys. Rev. D 65, 125029 (2002).

[47] J. Frenkel and J. C. Taylor, Nucl. Phys. B 334199 (1990); Nucl. Phys. B 374, 156 (1992).

[48] F. T. Brandt and J. Frenkel, Phys. Rev. D 474688 (1993); Phys. Rev. D 562453 (1997).

[49] F. T. Brandt, A. Das, O. Espinosa, J. Frenkel and S. Perez, Phys. Rev. D 72085006 (2005); Phys. Rev. D 73065010 (2006); Phys. Rev. D 73067702 (2006); Phys. Rev. D 74085006 (2006).

[50] M. Van Raamsdonk, JHEP 11, 006 (2001).

[51] A. Armoni and E. Lopez, Nucl. Phys. B 632, 240 (2002).

[52] G. Arcioni and M. A. Vazquez-Mozo, JHEP 01, 028 (2000).

[53] F. T. Brandt, J. Frenkel and C. Muramoto, Nucl. Phys. B 754146 (2006).

[54] F. T. Brandt, J. Frenkel and C. Muramoto, hep-th/0703242 (2007).

[55] G. E. Tauber and J. W. Weinberg, Phys. Rev. 1221342 (1961); N. A. Chernikov, Acta Phys. Pol. 23629 (1963).

[56] S. R. de Groot, W. A. van Leeuwen and Ch. G. van Weert, Relativistic Kinetic Theory, North Holland Publ. Co. (1980).

[57] E. Wigner, Phys. Rev. 40, 749 (1932).

[58] H. Th. Elze, M. Gyulassy and D. Vasak, Nucl. Phys. B 276, 706 (1986).

[59] H. Th. Elze, M. Gyulassy and D. Vasak, Phys. Lett. B 177, 3 (1986).

[60] H. Th. Elze and U. Heinz, Phys. Rep. 183, 81 (1989). (1986).

[61] D. F. Limim and C. Manuel, Phys. Rep. 364, 451 (2001).

[62] C. Manuel and S. Mrowczynski, Phys. Rev. D 67, 014015 (2003).

[63] A. V. Prozorkevich, S. A. Smolyansky and S. V. Ilyin, hep-th/0301169 (2004). 
[64] H. Th. Elze, hep-ph/0204309 (2002).

[65] I. S. Gradshteyn and M. Ryzhik, Tables of Integral Series and Products, Academic Press, New York, 1980.

[66] E. Butkov, Física Matemática. Ed. Guanabara Koogan S.A., Rio de Janeiro, 1988.

[67] J. Ambjorn, R. J. Hughes, Ann. Phys. 145340 (1983).

[68] F. T. Brandt, A. Das and J. Frenkel, Phys. Rev. D 66105012 (2002).

[69] F. T. Brandt, A. Das and J. Frenkel, Phys. Rev. D 68085010 (2003).

[70] J. Frenkel, S. H. Pereira, A. Das, F. T. Brandt and J. C. Taylor, Phys. Rev. D 67, 105010 (2003).

[71] J. Frenkel, S. H. Pereira, A. Das and J. C. Taylor, Phys. Lett. B 577, 76 (2003).

[72] J. Frenkel and S. H. Pereira, Phys. Rev. D 69, 127702 (2004).

[73] J. Frenkel, S. H. Pereira, A. Das and J. C. Taylor, Phys. Rev. A 70, 053408 (2004). 